\title{
Radical Perfluoroalkylation Enabled by a Catalytically Generated Halogen Bonding Complex and Visible Light Irradiation
}

Tarannum Tasnim, Calvin Ryan, Miranda L. Christensen, Christopher J. Fennell and Spencer P. Pitre*

Department of Chemistry, Oklahoma State University, 107 Physical Sciences, Stillwater, OK 74078, United States

Email: spencer.p.pitre@okstate.edu

Table of Contents

A. Materials and Methods $\quad$ S2

B. Picture of Photochemistry Set-Up $\quad$ S3

C. Full Reaction Optimization $\quad$ S4

D. Procedure for Control Reaction with TEMPO S6

E. General Procedure for Radical Perfluoroalkylation Reactions $\quad$ S7

F. Reaction Scope of the Radical Perfluoroalkylation Reaction S8

G. Procedure for 1 mmol Scale Radical Perfluoroalkylation Reaction $\quad$ S21

H. Procedure for Radical Perfluoroalkylation Reaction with $\mathbf{C}_{\mathbf{4}} \mathbf{F} \mathbf{9 B r} \quad \mathrm{S} 22$

I. UV-Vis Spectra of 2,5-Di-tert-butylhydroquinone $\quad$ S23

J. Benesi-Hildebrand Analysis of Charge-Transfer Complex $\quad$ S24

K. ${ }^{1}$ H NMR Spectra of 2,5-Di-tert-butylhydroquinone $\quad$ S26

$\begin{array}{ll}\text { L. Computational Studies } & \text { S27 }\end{array}$

M. References $\quad$ S35

$\begin{array}{lr}\text { N. NMR Spectra } & \text { S37 }\end{array}$ 


\section{A. Materials and Methods}

All reactions were conducted in oven-dried glassware under an atmosphere of argon, unless otherwise stated. All solvents, reagents and starting materials were purchased from commercial suppliers (Fisher Scientific, TCI America, Sigma Aldrich, Oakwood Chemicals, Combi-Blocks Inc.) and were used as received unless otherwise noted. All photochemistry experiments were performed using two $40 \mathrm{~W}$ Kessil A160WE Tuna Sun white LEDs (6,000K to 9,000K) while being maintained at approximately $25^{\circ} \mathrm{C}$. Thin-layer chromatography (TLC) was conducted with silica gel 60 F254 pre-coated plates $(0.25 \mu \mathrm{m})$ and visualized by exposure to UV-light $(254 \mathrm{~nm})$ or potassium permanganate $\left(\mathrm{KMnO}_{4}\right)$ staining. Flash column chromotagraphy was performed using a Biotage Isolera Four equipped with either Biotage SNAP, Biotage Sfär, or Sorbtech Purity flash column cartridges. Preparative thin layer chromatography was performed using Sorbtech silica gel prep TLC plates (w/UV254, glass backed, thickness: $1000 \mu \mathrm{m}$, dimensions: $20 \times 20 \mathrm{~cm}$ ). ${ }^{1} \mathrm{H}$ NMR spectra were recorded at $400 \mathrm{MHz}$ and are reported relative to deuterated solvent signals. Data for ${ }^{1} \mathrm{H}$ NMR spectra are reported as follows: chemical shift ( $\left.\delta \mathrm{ppm}\right)$, multiplicity, coupling constant $(\mathrm{Hz})$, and integration. ${ }^{13} \mathrm{C}$ NMR spectra were recorded at 101 or $201 \mathrm{MHz}$. Data for ${ }^{13} \mathrm{C}$ NMR spectra are reported in terms of chemical shift. ${ }^{19} \mathrm{~F}$ NMR spectra were recorded at $376 \mathrm{MHz}$. Data for ${ }^{19}$ F NMR spectra are reported as follows: chemical shift, multiplicity, coupling constant $(\mathrm{Hz})$, and integration. IR spectra were recorded on a Shimadzu IRAffinity-1S FT-IR spectrophotomer equipped with a QATR 10 single reflectance ATR accessory and are reported in terms of frequency of absorption $\left(\mathrm{cm}^{-1}\right)$. High-resolution mass spectra were obtained with a quadrupole-Orbitrap hybrid mass spectrometer at Oklahoma State University or a LCT spectrometer at UC Irvine. UVVis spectra were recorded on a Shimadzu UV-2600 UV-Vis spectrophotometer. 


\section{B. Picture of Photochemistry Setup}

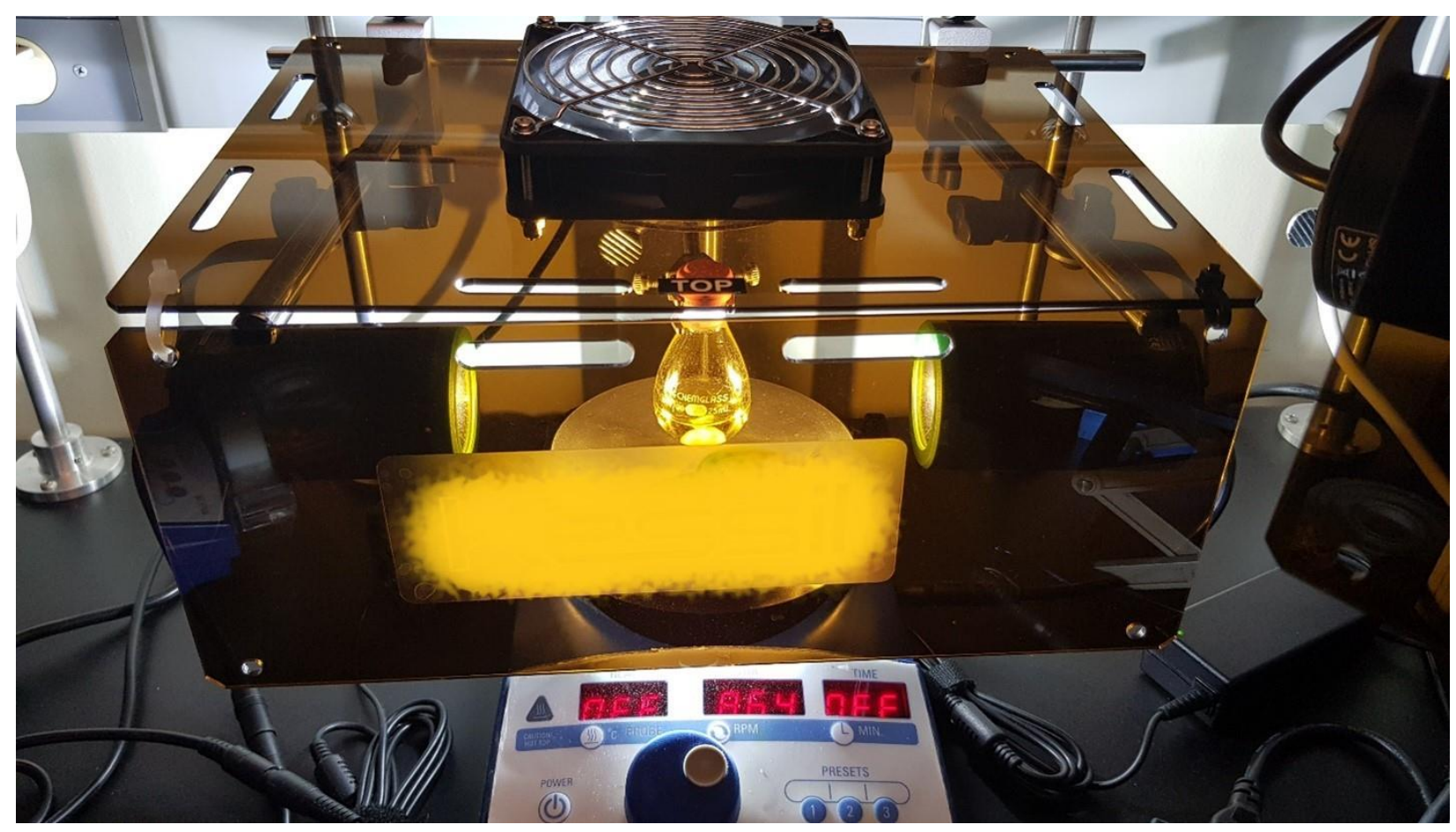

Figure S1. Picture of the photochemistry set-up employed in this work (1 mmol scale reaction shown). 


\section{Full Reaction Optimization}

Table S1. Optimization and control reactions for the perfluoroalkylation of caffeine.

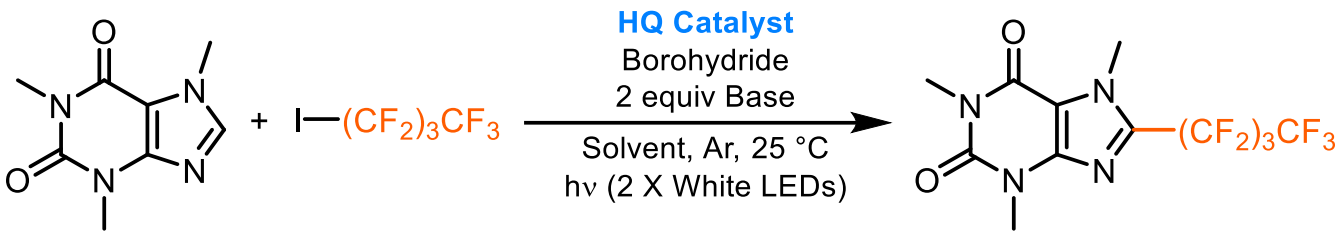

$0.15 \mathrm{mmol}$

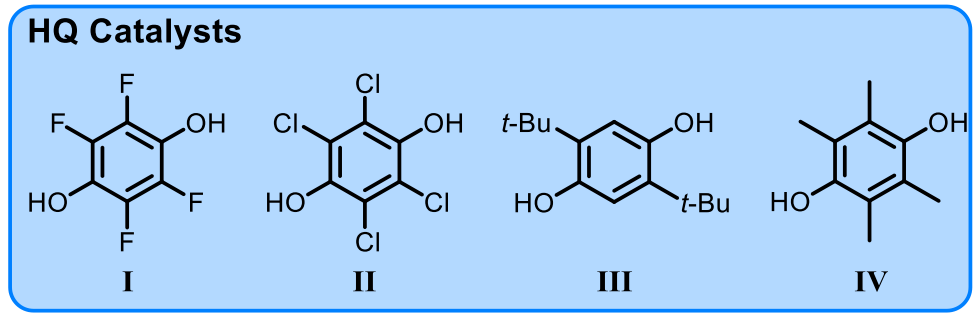

\begin{tabular}{|c|c|c|c|c|c|c|}
\hline $\begin{array}{c}\text { Borohydride } \\
(\mathrm{mol} \%)\end{array}$ & HQ (mol\%) & Base & $\begin{array}{l}\text { equiv } \\
\mathrm{C}_{4} \mathrm{~F}_{9} \mathrm{I}\end{array}$ & Solvent (Conc.) & Time & Yield $^{[a]}$ \\
\hline None & $\mathrm{I}(50)$ & $\mathrm{NaHCO}_{3}$ & 3 & $\operatorname{DMF}(0.15 \mathrm{M})$ & $18 \mathrm{~h}$ & Trace \\
\hline None & II (50) & $\mathrm{NaHCO}_{3}$ & 3 & $\operatorname{DMF}(0.15 \mathrm{M})$ & $18 \mathrm{~h}$ & Trace \\
\hline None & III (50) & $\mathrm{NaHCO}_{3}$ & 3 & $\operatorname{DMF}(0.15 \mathrm{M})$ & $18 \mathrm{~h}$ & $25 \%$ \\
\hline None & IV (50) & $\mathrm{NaHCO}_{3}$ & 3 & $\operatorname{DMF}(0.15 \mathrm{M})$ & $18 \mathrm{~h}$ & $21 \%$ \\
\hline None & III (50) & $\mathrm{NaHCO}_{3}$ & 3 & Methanol $(0.15 \mathrm{M})$ & $24 \mathrm{~h}$ & $0 \%$ \\
\hline None & III (50) & $\mathrm{NaHCO}_{3}$ & 3 & $\operatorname{MeCN}(0.15 \mathrm{M})$ & $24 \mathrm{~h}$ & $0 \%$ \\
\hline None & III (50) & $\mathrm{NaHCO}_{3}$ & 3 & 9:1 MeCN:MeOH (0.15 M) & $24 \mathrm{~h}$ & $45 \%$ \\
\hline $\mathrm{NaBH}_{4}(50)$ & III (50) & $\mathrm{NaHCO}_{3}$ & 3 & 9:1 MeCN:MeOH (0.15 M) & $24 \mathrm{~h}$ & $56 \%$ \\
\hline $\mathrm{NaCNBH}_{4}(50)$ & III (50) & $\mathrm{NaHCO}_{3}$ & 3 & 9:1 MeCN:MeOH (0.15 M) & $24 \mathrm{~h}$ & $46 \%$ \\
\hline STAB (50) & III (50) & $\mathrm{NaHCO}_{3}$ & 3 & 9:1 MeCN:MeOH (0.15 M) & $24 \mathrm{~h}$ & $62 \%$ \\
\hline None & III (50) & $\mathrm{NaHCO}_{3}$ & 3 & 9:1 MeCN:MeOH (0.15 M) & $24 \mathrm{~h}$ & $57 \%$ \\
\hline STAB (16.6) & III (50) & $\mathrm{NaHCO}_{3}$ & 3 & 9:1 MeCN:MeOH (0.15 M) & $24 \mathrm{~h}$ & $63 \%$ \\
\hline STAB (33.3) & III (50) & $\mathrm{NaHCO}_{3}$ & 3 & 9:1 MeCN:MeOH (0.15 M) & $24 \mathrm{~h}$ & $60 \%$ \\
\hline STAB (100) & III (50) & $\mathrm{NaHCO}_{3}$ & 3 & 9:1 MeCN:MeOH (0.15 M) & $24 \mathrm{~h}$ & $42 \%$ \\
\hline STAB (16.6) & III (100) & $\mathrm{NaHCO}_{3}$ & 3 & 9:1 MeCN:MeOH (0.15 M) & $24 \mathrm{~h}$ & $42 \%$ \\
\hline STAB (16.6) & III (33.3) & $\mathrm{NaHCO}_{3}$ & 3 & 9:1 MeCN:MeOH (0.15 M) & $24 \mathrm{~h}$ & $61 \%$ \\
\hline STAB (16.6) & III (16.6) & $\mathrm{NaHCO}_{3}$ & 3 & 9:1 MeCN:MeOH (0.15 M) & $24 \mathrm{~h}$ & $63 \%$ \\
\hline STAB (16.6) & III (10) & $\mathrm{NaHCO}_{3}$ & 3 & 9:1 MeCN:MeOH (0.15 M) & $24 \mathrm{~h}$ & $40 \%$ \\
\hline STAB (16.6) & III (5) & $\mathrm{NaHCO}_{3}$ & 3 & 9:1 MeCN:MeOH (0.15 M) & $24 \mathrm{~h}$ & $45 \%$ \\
\hline STAB (16.6) & III (16.6) & $\mathrm{K}_{2} \mathrm{CO}_{3}$ & 3 & 9:1 MeCN:MeOH (0.15 M) & $24 \mathrm{~h}$ & $53 \%$ \\
\hline STAB (16.6) & III (16.6) & $\mathrm{K}_{3} \mathrm{PO}_{4}$ & 3 & 9:1 MeCN:MeOH (0.15 M) & $24 \mathrm{~h}$ & $54 \%$ \\
\hline STAB (16.6) & III (16.6) & $\mathrm{Cs}_{2} \mathrm{CO}_{3}$ & 3 & 9:1 MeCN:MeOH (0.15 M) & $24 \mathrm{~h}$ & $12 \%$ \\
\hline STAB (50) & III (10) & $\mathrm{NaHCO}_{3}$ & 3 & 9:1 MeCN:MeOH (0.15 M) & $24 \mathrm{~h}$ & $60 \%$ \\
\hline STAB (50) & III (10) & $\mathrm{NaHCO}_{3}$ & 2 & 9:1 MeCN:MeOH (0.15 M) & $24 \mathrm{~h}$ & $35 \%$ \\
\hline STAB (50) & III (10) & $\mathrm{NaHCO}_{3}$ & 1 & 9:1 MeCN:MeOH (0.15 M) & $24 \mathrm{~h}$ & $26 \%$ \\
\hline STAB (50) & III (10) & $\mathrm{NaHCO}_{3}$ & 3 & 8:1 MeCN:MeOH (0.15 M) & $24 \mathrm{~h}$ & $49 \%$ \\
\hline STAB (50) & III (10) & $\mathrm{NaHCO}_{3}$ & 3 & 9:2 MeCN:MeOH (0.15 M) & $24 \mathrm{~h}$ & $55 \%$ \\
\hline STAB (50) & III (10) & $\mathrm{NaHCO}_{3}$ & 3 & 9:3 MeCN:MeOH (0.15 M) & $24 \mathrm{~h}$ & $49 \%$ \\
\hline STAB (50) & III (10) & $\mathrm{NaHCO}_{3}$ & 3 & 10:1 MeCN:MeOH (0.15 M) & $24 \mathrm{~h}$ & $59 \%$ \\
\hline STAB (50) & III (10) & $\mathrm{NaHCO}_{3}$ & 3 & 11:1 MeCN:MeOH (0.15 M) & $24 \mathrm{~h}$ & $63 \%$ \\
\hline
\end{tabular}




\begin{tabular}{|c|c|c|c|c|c|c|}
\hline STAB (50) & III (10) & $\mathrm{NaHCO}_{3}$ & 3 & 12:1 MeCN:MeOH (0.15 M) & $24 \mathrm{~h}$ & $60 \%$ \\
\hline STAB (50) & III (10) & $\mathrm{NaHCO}_{3}$ & 3 & 13:1 MeCN:MeOH (0.15 M) & $24 \mathrm{~h}$ & $57 \%$ \\
\hline STAB (50) & III (10) & $\mathrm{NaHCO}_{3}$ & 3 & 14:1 MeCN:MeOH (0.15 M) & $24 \mathrm{~h}$ & $50 \%$ \\
\hline STAB (50) & III (10) & $\mathrm{NaHCO}_{3}$ & 3 & 11:1 MeCN:MeOH (0.3 M) & $24 \mathrm{~h}$ & $45 \%$ \\
\hline STAB (50) & III (10) & $\mathrm{NaHCO}_{3}$ & 3 & 11:1 MeCN:MeOH (0.2 M) & $24 \mathrm{~h}$ & $58 \%$ \\
\hline STAB (50) & III (10) & $\mathrm{NaHCO}_{3}$ & 3 & 11:1 MeCN:MeOH (0.15 M) & $1 \mathrm{~h}$ & $5 \%$ \\
\hline STAB (50) & III (10) & $\mathrm{NaHCO}_{3}$ & 3 & 11:1 MeCN:MeOH (0.15 M) & $18 \mathrm{~h}$ & $54 \%$ \\
\hline None & III (10) & $\mathrm{NaHCO}_{3}$ & 3 & 11:1 MeCN:MeOH (0.15 M) & $30 \mathrm{~h}^{[\mathrm{b}]}$ & $70 \%$ \\
\hline None & III (10) & $\mathrm{NaHCO}_{3}$ & 3 & 11:1 MeCN:MeOH (0.15 M) & $36 h^{[b]}$ & $70 \%$ \\
\hline STAB (50) & III (10) & $\mathrm{NaHCO}_{3}$ & 3 & 11:1 MeCN:MeOH (0.15 M) & $24 \mathrm{~h}$ & $8 \%{ }^{[\mathrm{c}]}$ \\
\hline STAB (50) & III (10) & None & 3 & 11:1 MeCN:MeOH (0.15 M) & $24 \mathrm{~h}$ & $22 \%$ \\
\hline None & III (10) & $\mathrm{NaHCO}_{3}$ & 3 & 11:1 MeCN:MeOH (0.15 M) & $24 \mathrm{~h}$ & $61 \%$ \\
\hline STAB (50) & None & $\mathrm{NaHCO}_{3}$ & 3 & 11:1 MeCN:MeOH (0.15 M) & $24 \mathrm{~h}$ & Trace \\
\hline STAB (50) & III (10) & $\mathrm{NaHCO}_{3}$ & 3 & 11:1 MeCN:MeOH (0.15 M) & $24 \mathrm{~h}$ & Trace $^{[\mathrm{d}}$ \\
\hline
\end{tabular}

${ }^{[\mathrm{a}]}$ Yields were determined by ${ }^{19} \mathrm{~F}$ NMR using 4-fluorobenzoic acid as an external standard. ${ }^{[b]}$ Reaction carried out at $0.3 \mathrm{mmol}$ scale. ${ }^{[\mathrm{c}]}$ No degassing with Ar. ${ }^{[\mathrm{d}]}$ No light. 


\section{Procedure for Control Reaction with TEMPO}

An oven-dried two-dram borosilicate glass vial equipped with a magnetic stir bar was charged with caffeine ( $58 \mathrm{mg}, 0.3 \mathrm{mmol}, 1$ equiv), perfluorobutyl iodide ( $156 \mu \mathrm{L}, 0.9 \mathrm{mmol}, 3$ equiv), 2,5di-tert-butylhydroquinone (7.0 mg, $0.03 \mathrm{mmol}, 10 \mathrm{~mol} \%$ ) and $\mathrm{NaHCO}_{3}(50.0 \mathrm{mg}, 0.6 \mathrm{mmol}, 2$ equiv). 11:1 MeCN:MeOH was added ( $2 \mathrm{~mL}, 0.15 \mathrm{M})$, and the reaction mixture was degassed by sparging with argon for 5-6 minutes. The reaction mixture was then sonicated and irradiated with two Kessil A160WE Tuna Sun LED lamps for $24 \mathrm{~h}$ at $25^{\circ} \mathrm{C}$. The reaction mixture was transferred into a separatory funnel and diluted with $15 \mathrm{~mL}$ of DCM and washed with $15 \mathrm{~mL}$ of $10 \mathrm{mM}$ $\mathrm{Na}_{2} \mathrm{~S}_{2} \mathrm{O}_{3}$ (aq). The aqueous phase was extracted with $15 \mathrm{~mL}$ of DCM. The combined organic phases were dried with $\mathrm{MgSO}_{4}$ and concentrated. Yield was calculated from ${ }^{19} \mathrm{~F}$ NMR using 4fluorobenzoic acid $(12.6 \mathrm{mg}$ ) as an external standard (see Figure S2). The calculation showed only trace amount of product formation after $24 \mathrm{~h}$. The yield for the TEMPO- $\mathrm{C}_{4} \mathrm{~F}_{9}$ adduct was calculated as $14 \%$.

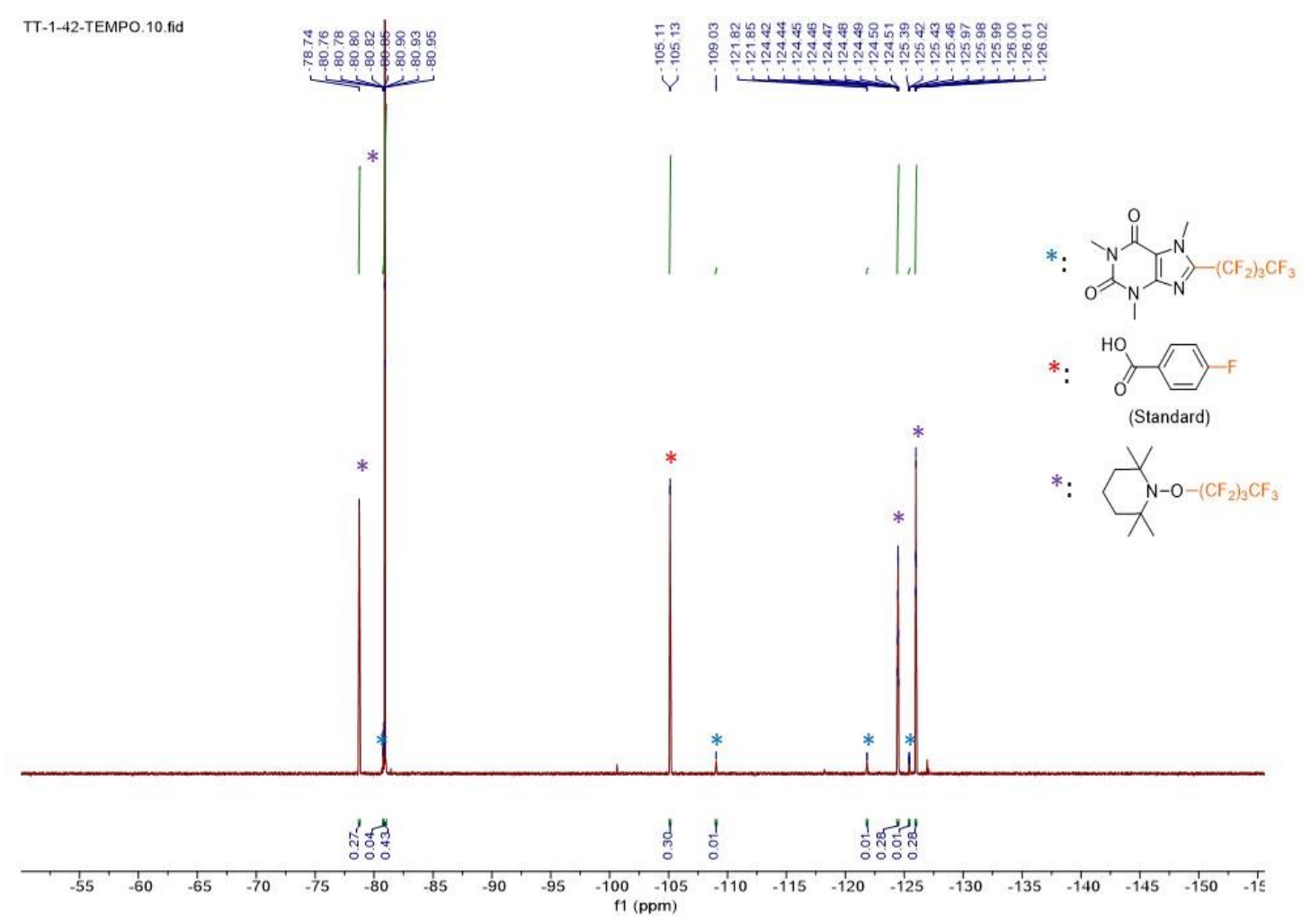

Figure S2. ${ }^{19}$ F NMR spectrum of the crude material for the control reaction with TEMPO. 


\section{E. General Procedure for Radical Perfluoroalkylation Reactions}

An oven-dried two-dram borosilicate glass vial equipped with a magnetic stir bar was charged with a (hetero)arene ( $0.3 \mathrm{mmol}, 1$ equiv), perfluoroalkyl iodide ( $0.9 \mathrm{mmol}, 3$ equiv), 2,5-di-tertbutylhydroquinone (7.0 mg, $0.03 \mathrm{mmol}, 10 \mathrm{~mol} \%)$ and $\mathrm{NaHCO}_{3}(50.0 \mathrm{mg}, 0.6 \mathrm{mmol}, 2$ equiv). 11:1 MeCN:MeOH was added ( $2 \mathrm{~mL}, 0.15 \mathrm{M})$, and the reaction mixture was degassed by sparging with argon for 5-6 minutes. The reaction mixture was then sonicated and irradiated with two Kessil A160WE Tuna Sun LED lamps for $30 \mathrm{~h}$ at $25{ }^{\circ} \mathrm{C}$. The reaction mixture was transferred into a separatory funnel and diluted with $15 \mathrm{~mL}$ of DCM and washed with $15 \mathrm{~mL}$ of $10 \mathrm{mM} \mathrm{Na} 2 \mathrm{~S}_{2} \mathrm{O}_{3}$ (aq). The aqueous phase was extracted with $15 \mathrm{~mL}$ of DCM. The combined organic phases were dried with $\mathrm{MgSO}_{4}$ and concentrated. Purification was performed by flash column chromatography using a Biotage Isolera Four. 


\section{F. Reaction Scope of the Radical Perfluoroalkylation Reaction}

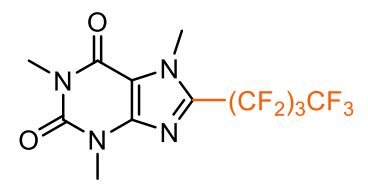

1,3,7-Trimethyl-8-(perfluorobutyl)-3,7-dihydro-1H-purine-2,6-dione

(1): Prepared according to the general procedure from caffeine $(58 \mathrm{mg}, 0.3$ mmol), perfluorobutyl iodide (156 $\mu \mathrm{L}, 0.9 \mathrm{mmol}, 3$ equiv), 2,5-di-tertbutylhydroquinone (7.0 mg, $0.03 \mathrm{mmol}, 10 \mathrm{~mol} \%$ ) and $\mathrm{NaHCO}_{3}(50 \mathrm{mg}, 0.6 \mathrm{mmol}, 2$ equiv) in 2 $\mathrm{mL}$ of 11:1 MeCN:MeOH. The reaction was irradiated with two Kessil A160WE Tuna Sun LEDs for $30 \mathrm{~h}$ at $25^{\circ} \mathrm{C}$. Purified by flash column chromatography $(0 \rightarrow 20 \%$ EtOAc in Hex $)$ to give the title compound as a white solid in $67 \%$ yield $(82 \mathrm{mg})$.

${ }^{1} \mathbf{H}$ NMR: $\left(400 \mathrm{MHz}, \mathrm{CDCl}_{3}\right) \delta 4.20-4.17(\mathrm{~m}, 3 \mathrm{H}), 3.59(\mathrm{~s}, 3 \mathrm{H}), 3.42(\mathrm{~s}, 3 \mathrm{H}) .{ }^{13} \mathbf{C}$ NMR: $(201$ $\left.\mathrm{MHz}, \mathrm{CDCl}_{3}\right) \delta 155.6,151.4,147.1,137.9(\mathrm{t}, J=28.9 \mathrm{~Hz}), 117.4(\mathrm{qt}, J=289.1,34.3 \mathrm{~Hz}), 113.3$ - 107.2 (m), 110.3, 34.0, 30.0, 28.3. ${ }^{19}$ F NMR: $\left(376 \mathrm{MHz}, \mathrm{CDCl}_{3}\right) \delta-80.81$ - -80.95 (m, 3F), 109.08 (m, 2F), -121.88 (m, 2F), -125.40 - -125.56 (m, 2F). Rf: 0.56 (2:1 Hex:EtOAc) Reference: Org. Biomol. Chem. 2016, 14, 6417-6421.

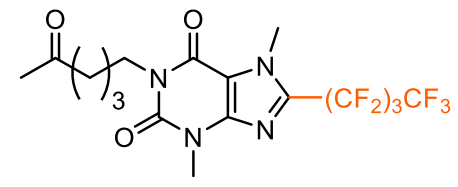

3,7-Dimethyl-1-(5-oxohexyl)-8-(perfluorobutyl)-3,7-dihydro-1Hpurine-2,6-dione (2): Prepared according to the general procedure from pentoxyphylline ( $83 \mathrm{mg}, 0.3 \mathrm{mmol}$ ), perfluorobutyl iodide (156 $\mu \mathrm{L}, 0.9 \mathrm{mmol}, 3$ equiv), 2,5-di-tert-butylhydroquinone (7.0 mg, $0.03 \mathrm{mmol}, 10 \mathrm{~mol} \%)$ and $\mathrm{NaHCO}_{3}(50 \mathrm{mg}, 0.6 \mathrm{mmol}, 2$ equiv) in $2 \mathrm{~mL}$ of 11:1 MeCN:MeOH. The reaction was irradiated with two Kessil A160WE Tuna Sun LEDs for $30 \mathrm{~h}$ at $25{ }^{\circ} \mathrm{C}$. Purified by flash column chromatography $(0 \rightarrow 20 \%$ EtOAc in Hex) to give the title compound as a white solid in $75 \%$ yield (112 mg).

${ }^{1}$ H NMR: (400 MHz, $\left.\mathrm{CDCl}_{3}\right) \delta 4.19-4.15(\mathrm{~m}, 3 \mathrm{H}), 4.01(\mathrm{t}, J=6.9 \mathrm{~Hz}, 2 \mathrm{H}), 3.56(\mathrm{~s}, 3 \mathrm{H}), 2.49(\mathrm{t}$, $J=6.8 \mathrm{~Hz}, 2 \mathrm{H}), 2.13(\mathrm{~s}, 3 \mathrm{H}), 1.72-1.60$ (m, 4H). $\left.{ }^{13} \mathrm{C} \mathrm{NMR:} \mathrm{(201} \mathrm{MHz,} \mathrm{CDCl}_{3}\right) \delta 208.7,155.4$, 151.1, 147.2, 137.9 (t, $J=28.8$ Hz), 119.6 - $115.3(\mathrm{~m}), 113.3$ - 108.5 (m), 110.3, 43.2, 41.3, 34.0, 30.1, 30.0, 27.5, 21.0. ${ }^{19}$ F NMR: $\left(376 \mathrm{MHz}, \mathrm{CDCl}_{3}\right) \delta-75.81--88.44(\mathrm{~m}, 3 \mathrm{~F}),-109.06(\mathrm{t}, J=$ 13.2 Hz, 2F), -121.81 - -121.90 (m, 2F), -125.42 - -125.50 (m, 2F). Rf: 0.28 (2:1 Hex:EtOAc) HRMS (ESI) m/z: $[\mathrm{M}+\mathrm{H}]^{+}$calculated for $\mathrm{C}_{17} \mathrm{H}_{17} \mathrm{~F}_{9} \mathrm{~N}_{4} \mathrm{O}_{3}$ 497.1235; found 497.1225. IR (neat, $\mathbf{c m}^{-1}$ ): 2958, 2926, 2854, 1704, 1664, 1612, 1544, 1495, 1429, 1350, 1266, 1231, 1151, 1063, 967 , $942,828,768,746,721,654,533$. 


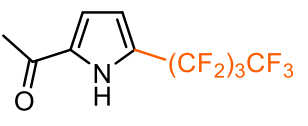

1-(5-(Perfluorobutyl)-1H-pyrrol-2-yl)ethan-1-one (3): Prepared according to the general procedure from 2-acetylpyrrole (33 $\mathrm{mg}, 0.3 \mathrm{mmol}$ ), perfluorobutyl iodide ( $156 \mu \mathrm{L}, 0.9 \mathrm{mmol}, 3$ equiv), 2,5-di-tert-butylhydroquinone ( $7.0 \mathrm{mg}, 0.03$ mmol, $10 \mathrm{~mol} \%$ ) and $\mathrm{NaHCO}_{3}(50 \mathrm{mg}, 0.6 \mathrm{mmol}, 2$ equiv) in $2 \mathrm{~mL}$ of 11:1 MeCN:MeOH. The reaction was irradiated with two Kessil A160WE Tuna Sun LEDs for $30 \mathrm{~h}$ at $25^{\circ} \mathrm{C}$. Purified by flash column chromatography (Hex $\rightarrow$ 9:1 Hex:EtOAc) to give the title compound as a pale-yellow solid in $60 \%$ yield $(59 \mathrm{mg})$.

${ }^{1}$ H NMR: (400 MHz, $\left.\mathrm{CDCl}_{3}\right) \delta 9.72$ (b.s, 1H), $6.93-6.89$ (m, 1H), $6.67-6.60$ (m, 1H), 2.49 (s, 3H). ${ }^{13}$ C NMR: $\left(201 \mathrm{MHz}, \mathrm{CDCl}_{3}\right) \delta 188.8,134.5,124.1(\mathrm{t}, J=30.6 \mathrm{~Hz}), 117.5$ (qt, $J=288.5$, $33.4 \mathrm{~Hz}), 116.1,115.7-114.8(\mathrm{~m}), 114.1-108.7(\mathrm{~m}), 112.7(\mathrm{t}, J=32.4 \mathrm{~Hz}), 25.8 .{ }^{19} \mathbf{F}$ NMR: $\left(376 \mathrm{MHz}, \mathrm{CDCl}_{3}\right) \delta-81.04$ (m, 3F), -109.12--109.30 (m, 2F), -123.19--123.35 (m, 2F), -125.80 (m, 2F). Rf: 0.49 (4:1 Hex:EtOAc) HRMS (ESI) m/z: could not be obtained due to difficulties ionizing the compound. IR (neat, $\mathbf{c m}^{-1}$ ): 3259, 1662, 1559, 1498, 1442, 1352, 1271, 1231, 1203 , 1131, 1099, 1051, 962, 950, 854, 797, 745, 735, 574.

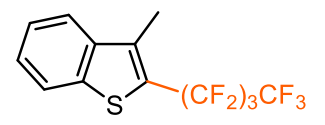

3-Methyl-2-(perfluorobutyl)benzo[b]thiophene (4): Prepared according to the general procedure from 3-methylbenzo[b]thiophene (39 $\mathrm{mg}, 0.3 \mathrm{mmol})$, perfluorobutyl iodide (156 $\mu \mathrm{L}, 0.9 \mathrm{mmol}, 3$ equiv), 2,5-di-tert-butylhydroquinone (7.0 mg, 0.03 mmol, $10 \mathrm{~mol} \%$ ) and $\mathrm{NaHCO}_{3}(50 \mathrm{mg}, 0.6 \mathrm{mmol}, 2$ equiv) in $2 \mathrm{~mL}$ of 11:1 MeCN:MeOH. The reaction was irradiated with two Kessil A160WE Tuna Sun LEDs for $30 \mathrm{~h}$ at $25{ }^{\circ} \mathrm{C}$. Purified by preparative thin layer chromatography (PTLC) using 9:1 Hex:EtOAc to give the title compound as a colorless oil in $58 \%$ yield $(64 \mathrm{mg})$.

${ }^{1}$ H NMR: $\left(400 \mathrm{MHz}, \mathrm{CDCl}_{3}\right) \delta 7.90-7.78(\mathrm{~m}, 2 \mathrm{H}), 7.53-7.38(\mathrm{~m}, 2 \mathrm{H}), 2.56(\mathrm{~s}, 3 \mathrm{H}) .{ }^{13} \mathbf{C}$ NMR: $\left(201 \mathrm{MHz}, \mathrm{CDCl}_{3}\right) \delta$ 140.0, 139.7, $136.9(\mathrm{t}, J=4.0 \mathrm{~Hz}), 127.7-107.7(\mathrm{~m}), 126.8,124.9,123.3$, 122.6, 12.6. ${ }^{19}$ F NMR: $\left(376 \mathrm{MHz}, \mathrm{CDCl}_{3}\right) \delta-80.60--81.22(\mathrm{~m}, 3 \mathrm{~F}),-100.39--100.57$ (m, 2F), -121.47 - -121.67 (m, 2F), -125.65 - -125.85 (m, 2F). Rf: 0.82 (9:1 Hex:EtOAc) HRMS (GC CI) $\mathbf{m} / \mathbf{z}: \mathrm{M}^{+}$calculated for $\mathrm{C}_{13} \mathrm{H}_{7} \mathrm{~F}_{9} \mathrm{~S}$ 366.0125; found 366.0121. IR (neat, $\mathbf{c m}^{-1}$ ): 3074, 2930, 1352, 1272, 1231, 1132, 1098, 1071, 1012, 980, 968, 854, 786, 755, 732, 531. 


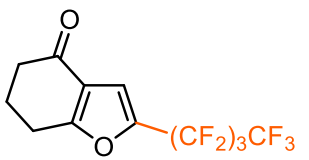
according to the general procedure from 6,7-dihydro-4(5H)benzofuranone ( 35 $\mu \mathrm{L}, 0.3 \mathrm{mmol})$, perfluorobutyl iodide ( $156 \mu \mathrm{L}, 0.9 \mathrm{mmol}, 3$ equiv), 2,5-di-tertbutylhydroquinone ( $7.0 \mathrm{mg}, 0.03 \mathrm{mmol}, 10 \mathrm{~mol} \%$ ) and $\mathrm{NaHCO}_{3}(50 \mathrm{mg}, 0.6 \mathrm{mmol}, 2$ equiv) in 2 $\mathrm{mL}$ of 11:1 MeCN:MeOH. The reaction was irradiated with two Kessil A160WE Tuna Sun LEDs for $30 \mathrm{~h}$ at $25^{\circ} \mathrm{C}$. Purified by flash column chromatography (Hex $\rightarrow$ 9:1 Hex:EtOAc) to give the title compound as a yellow oil in $41 \%$ yield $(45 \mathrm{mg}$ ).

${ }^{1}$ H NMR: $\left(400 \mathrm{MHz}, \mathrm{CDCl}_{3}\right) \delta 7.13(\mathrm{~s}, 1 \mathrm{H}), 2.97(\mathrm{t}, J=6.3,2 \mathrm{H}), 2.58-2.52(\mathrm{~m}, 2 \mathrm{H}), 2.24(\mathrm{p}, J$ $=6.4 \mathrm{~Hz}, 2 \mathrm{H}) .{ }^{13} \mathrm{C}$ NMR: $\left(201 \mathrm{MHz}, \mathrm{CDCl}_{3}\right) \delta 193.4,169.5,141.4(\mathrm{t}, J=33.6 \mathrm{~Hz}), 122.0,120.2$ - $108.3(\mathrm{~m}), 111.1,37.7,23.5,22.3 .{ }^{19} \mathbf{F}$ NMR: $\left(376 \mathrm{MHz}, \mathrm{CDCl}_{3}\right) \delta-80.93--81.00(\mathrm{~m}, 3 \mathrm{~F}),-$ 111.52 (t, $J=12.3 \mathrm{~Hz}, 2 \mathrm{~F}),-123.29--123.42$ (m, 2F), -125.96 - -126.08 (m, 2F). Rf: 0.50 (4:1 Hex:EtOAc) HRMS (ESI) m/z: [M+H] $]^{+}$calculated for $\mathrm{C}_{12} \mathrm{H}_{7} \mathrm{~F}_{9} \mathrm{O}_{2}$ 355.0380; found 355.0385. IR (neat, $\mathbf{c m}^{-1}$ ): 2960, 1693 , 1605, 1570, 1458, 1416, 1352, 1204, 1130, 1091, 950, 939, 858, 738, $725,533$.

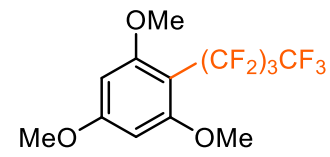

1,3,5-Trimethoxy-2-(perfluorobutyl)benzene (6): Prepared according to the general procedure from 1,3,5-trimethoxybenzene $(58 \mathrm{mg}, 0.3 \mathrm{mmol})$, perfluorobutyl iodide (156 $\mu \mathrm{L}, 0.9 \mathrm{mmol}, 3$ equiv), 2,5-di-tertbutylhydroquinone ( $7.0 \mathrm{mg}, 0.03 \mathrm{mmol}, 10 \mathrm{~mol} \%)$ and $\mathrm{NaHCO}_{3}(50 \mathrm{mg}, 0.6 \mathrm{mmol}, 2$ equiv) in 2 $\mathrm{mL}$ of 11:1 MeCN:MeOH. The reaction was irradiated with two Kessil A160WE Tuna Sun LEDs for $30 \mathrm{~h}$ at $25{ }^{\circ} \mathrm{C}$. Purified by flash column chromatography $(0 \rightarrow 15 \%$ EtOAc in Hex) to give the title compound as a white solid in $89 \%$ yield (103 mg).

${ }^{1}$ H NMR: $\left(400 \mathrm{MHz}, \mathrm{CDCl}_{3}\right) \delta 6.14$ (s, 2H), 3.84 (s, 3H), 3.80 (s, 6H). ${ }^{13} \mathbf{C}$ NMR: $(201 \mathrm{MHz}$, $\left.\mathrm{CDCl}_{3}\right) \delta$ 164.0, 161.9, 120.2 - $115.7(\mathrm{~m}), 111.4-107.9(\mathrm{~m}), 98.7$ (t, $\left.J=21.8 \mathrm{~Hz}\right), 56.5$, 55.5. ${ }^{19}$ F NMR: $\left(376 \mathrm{MHz}, \mathrm{CDCl}_{3}\right) \delta-80.94(\mathrm{~m}, 2 \mathrm{~F}),-102.74--102.90(\mathrm{~m}, 2 \mathrm{~F}),-122.88--123.07(\mathrm{~m}$, 2F), -126.34 - -126.49 (m, 2F). Rf: 0.33 (2:1 Hex:EtOAc) Reference: Cell. Rep. Phys. Sci. 2020, $1,100141$.

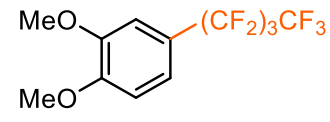

1,2-Dimethoxy-4-(perfluorobutyl)benzene (7): Prepared according to the general procedure from veratrole $(38 \mathrm{mg}, 0.3 \mathrm{mmol})$, perfluorobutyl iodide 
(156 $\mu \mathrm{L}, 0.9 \mathrm{mmol}, 3$ equiv), 2,5-di-tert-butylhydroquinone (7.0 mg, $0.03 \mathrm{mmol}, 10 \mathrm{~mol} \%$ ) and $\mathrm{NaHCO}_{3}(50 \mathrm{mg}, 0.6 \mathrm{mmol}, 2$ equiv) in $2 \mathrm{~mL}$ of 11:1 MeCN:MeOH. The reaction was irradiated with two Kessil A160WE Tuna Sun LEDs for $30 \mathrm{~h}$ at $25{ }^{\circ} \mathrm{C}$. Purified by flash column chromatography (Hex $\rightarrow$ 9:1 Hex:EtOAc) to give the title compound as a white solid in $70 \%$ yield (75 mg).

${ }^{1}$ H NMR: (400 MHz, $\left.\mathrm{CDCl}_{3}\right) \delta 7.20$ - $7.15(\mathrm{~m}, 1 \mathrm{H}), 7.04-7.00(\mathrm{~m}, 1 \mathrm{H}), 6.97-6.93(\mathrm{~m}, 1 \mathrm{H})$, 3.93 (s, 3H), 3.92 (s, 3H). ${ }^{13}$ C NMR: (201 MHz, $\left.\mathrm{CDCl}_{3}\right) \delta 152.0,149.1,121.0(\mathrm{t}, J=25.0 \mathrm{~Hz})$, $120.3(\mathrm{t}, J=7.0 \mathrm{~Hz}), 120.0-114.4(\mathrm{~m}), 110.8,109.5(\mathrm{t}, J=6.6 \mathrm{~Hz}), 56.1(\mathrm{~d}, J=12.7 \mathrm{~Hz}) .{ }^{19} \mathbf{F}$ NMR: $\left(376 \mathrm{MHz}, \mathrm{CDCl}_{3}\right) \delta-81.08--81.01$ (m, 3F), -109.80- -109.93 (m, 2F), -122.79- -122.70 (m, 2F), -125.45 - -125.76 (m, 2F). Rf: 0.56 (4:1 Hex:EtOAc) HRMS (GC CI) m/z: [M+H] ${ }^{+}$ calculated for $\mathrm{C}_{12} \mathrm{H}_{9} \mathrm{~F}_{9} \mathrm{O}_{2}$ 357.0537; found 357.0529. IR (neat, $\mathbf{c m}^{-1}$ ): 3010, 2945, 2845, 1609, 1521, 1468, 1350, 1273, 1219, 1127, 1043, 922, 892, 863, 768, 749, 727, 533.

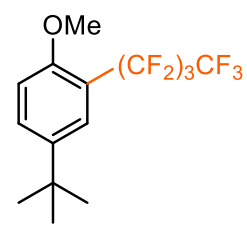

4-(Tert-butyl)-1-methoxy-2-(perfluorobutyl)benzene (8): Prepared according to the general procedure from 4-tert-butylanisole (53 $\mu \mathrm{L}, 0.3 \mathrm{mmol}$ ), perfluorobutyl iodide ( $156 \mu \mathrm{L}, 0.9 \mathrm{mmol}, 3$ equiv), 2,5-di-tert-butylhydroquinone (7.0 mg, $0.03 \mathrm{mmol}, 10 \mathrm{~mol} \%$ ) and $\mathrm{NaHCO}_{3}(50 \mathrm{mg}, 0.6 \mathrm{mmol}, 2$ equiv) in $2 \mathrm{~mL}$ of 11:1 MeCN:MeOH. The reaction was irradiated with two Kessil A160WE Tuna Sun LEDs for $30 \mathrm{~h}$ at $25^{\circ} \mathrm{C}$. Purified by flash column chromatography (Hex $\rightarrow$ 9:1 Hex:EtOAc) to give the title compound as a yellow oil in $77 \%$ yield $(87 \mathrm{mg})$.

${ }^{1}$ H NMR: $\left(400 \mathrm{MHz}, \mathrm{CDCl}_{3}\right) \delta 7.54-7.50(\mathrm{~m}, 1 \mathrm{H}), 7.49-7.47(\mathrm{~m}, 1 \mathrm{H}), 6.95(\mathrm{~d}, J=8.7 \mathrm{~Hz}, 1 \mathrm{H})$, $3.84(\mathrm{~s}, 3 \mathrm{H}), 1.32$ (s, 9H). ${ }^{13} \mathrm{C}$ NMR: $\left(201 \mathrm{MHz}, \mathrm{CDCl}_{3}\right) \delta 156.4,143.3,130.5,126.1(\mathrm{t}, J=8.8$ Hz), 118.2 (dt, $J=112.3,33.6 \mathrm{~Hz}), 117.2$ - 115.2 (m), 112.4, 111.6 - 110.3 (m), 109.9 - 108.8 (m), 56.2, 34.4, 31.4. ${ }^{19}$ F NMR: $\left(376 \mathrm{MHz} \mathrm{CDCl}_{3}\right) \delta-80.98(\mathrm{~m}, 3 \mathrm{~F}),-107.80(\mathrm{~m}, 2 \mathrm{~F}),-122.17$ (m, 2F), -125.99 - -126.17 (m, 2F). Rf: 0.33 (Hexane). HRMS (ESI) m/z: could not be obtained due to difficulties ionizing the compound. IR (neat, $\mathbf{c m}^{-1}$ ): 2966, 1617, 1507, 1350, 1275, 1229, 1130, 1074, 1033, 1010, 885, 839, 822, 746, 702, 693. 


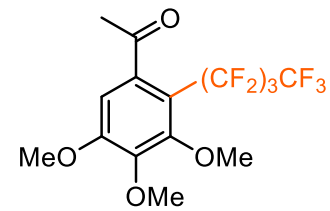

1-(3,4,5-Trimethoxy-2-(perfluorobutyl)phenyl)ethan-1-one (9): Prepared according to the general procedure from (3,4,5-trimethoxy)acetophenone (63 $\mathrm{mg}, 0.3 \mathrm{mmol})$, perfluorobutyl iodide ( $156 \mu \mathrm{L}, 0.9 \mathrm{mmol}, 3$ equiv), 2,5-ditert-butylhydroquinone (7.0 mg, $0.03 \mathrm{mmol}, 10 \mathrm{~mol} \%$ ) and $\mathrm{NaHCO}_{3}(50 \mathrm{mg}, 0.6 \mathrm{mmol}, 2$ equiv) in $2 \mathrm{~mL}$ of 11:1 MeCN:MeOH. The reaction was irradiated with two Kessil A160WE Tuna Sun LEDs for $30 \mathrm{~h}$ at $25^{\circ} \mathrm{C}$. Purified by flash column chromatography (Hex $\rightarrow$ 9:1 Hex:EtOAc) to give the title compound as a white solid in $45 \%$ yield $(57 \mathrm{mg})$.

${ }^{1}$ H NMR: (400 MHz, CDCl $) \delta 6.46(\mathrm{~s}, 1 \mathrm{H}), 3.92(\mathrm{~s}, 3 \mathrm{H}), 3.91$ (s, 3H), 3.87 (s, 3H), 2.49 (s, 3H). ${ }^{13}$ C NMR: $\left(201 \mathrm{MHz}, \mathrm{CDCl}_{3}\right) \delta 202.5,156.7,154.0(\mathrm{t}, J=3.7 \mathrm{~Hz}), 143.2,140.0(\mathrm{t}, J=4.0 \mathrm{~Hz})$, $120.1-115.9(\mathrm{~m}), 111.0(\mathrm{t}, J=23.1 \mathrm{~Hz}), 104.5,62.0,61.0,56.4,31.5 .{ }^{19}$ F NMR: $(376 \mathrm{MHz}$, $\left.\mathrm{CDCl}_{3}\right) \delta-80.70--80.98(\mathrm{~m}, 3 \mathrm{~F}),-99.83(\mathrm{t}, J=14.9 \mathrm{~Hz}, 2 \mathrm{~F}),-119.26--119.39(\mathrm{~m}, 2 \mathrm{~F}),-126.03$ - -126.35 (m, 2F). Rf: 0.69 (2:1 Hex:EtOAc). HRMS (ESI) m/z: $[\mathrm{M}+\mathrm{H}]^{+}$calculated for $\mathrm{C}_{15} \mathrm{H}_{13} \mathrm{~F}_{9} \mathrm{O}_{4}$ 429.0748; found 429.0739. IR (neat, $\mathbf{c m}^{-1}$ ): 2952, 1708, 1589, 1571, 1499, 1456, $1365,1265,1233,1186,1133,1055,1001,936,905,831,781,735,688,584$.

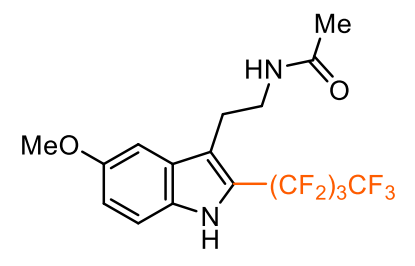

\section{$N$-(2-(5-Methoxy-2-(perfluorobutyl)-1H-indol-3-yl)ethyl)acetamide}

(10): Prepared according to the general procedure from melatonin (69 $\mathrm{mg}, 0.3 \mathrm{mmol}$ ), perfluorobutyl iodide ( $52 \mu \mathrm{L}, 0.3 \mathrm{mmol}, 1$ equiv), 2,5di-tert-butylhydroquinone (7.0 mg, $0.03 \mathrm{mmol}, 10 \mathrm{~mol} \%$ ) and $\mathrm{NaHCO}_{3}$ (50 mg, $0.6 \mathrm{mmol}, 2$ equiv) in $2 \mathrm{~mL}$ of 11:1 MeCN:MeOH. The reaction was irradiated with two Kessil A160WE Tuna Sun LEDs for $30 \mathrm{~h}$ at $25^{\circ} \mathrm{C}$. Purified by flash column chromatography (0$35 \% \mathrm{Hex} \rightarrow$ EtOAc) to give the title compound as colorless oil in $47 \%$ yield $(63 \mathrm{mg})$.

${ }^{1}$ H NMR: $\left(400 \mathrm{MHz}, \mathrm{CDCl}_{3}\right) \delta 8.68(\mathrm{~s}, 1 \mathrm{H}), 7.32(\mathrm{~d}, J=8.9 \mathrm{~Hz}, 1 \mathrm{H}), 7.17(\mathrm{~d}, J=2.4 \mathrm{~Hz}, 1 \mathrm{H})$, 6.99 (dd, $J=9.0,2.4 \mathrm{~Hz}, 1 \mathrm{H}), 5.67$ (b.s, 1H), 3.86 (s, 3H), 3.55 (q, $J=6.7 \mathrm{~Hz}, 2 \mathrm{H}), 3.07$ (t, $J=$ $7.1 \mathrm{~Hz}, 2 \mathrm{H}), 1.94$ (s, 3H). ${ }^{13} \mathrm{C}$ NMR: $\left(201 \mathrm{MHz}, \mathrm{CDCl}_{3}\right) \delta 170.5,155.0,131.5,128.2,120.4(\mathrm{t}, J$ $=28.7 \mathrm{~Hz}), 120.9-113.3(\mathrm{~m}), 117.3,116.7,112.9,100.7,55.9,40.3,24.5,23.4 .{ }^{19} \mathbf{F}$ NMR: $(376$ $\left.\mathrm{MHz}, \mathrm{CDCl}_{3}\right) \delta-80.80--81.12(\mathrm{~m}, 3 \mathrm{~F}),-107.48(\mathrm{t}, J=12.9 \mathrm{~Hz}, 2 \mathrm{~F}),-122.66--122.90(\mathrm{~m}, 2 \mathrm{~F}),-$ 125.75 - -126.03 (m, 2F). Rf: 0.15 (2:1 Hex:EtOAc) HRMS (ESI) m/z: $[\mathrm{M}+\mathrm{H}]^{+}$calculated for $\mathrm{C}_{17} \mathrm{H}_{15} \mathrm{~F}_{9} \mathrm{~N}_{2} \mathrm{O}_{2}$ 451.1068; found 451.1046. IR (neat, $\mathbf{~ c m}^{-1}$ ): 3257, 2926, 1654, 1559, 1466, 1437 , $1350,1233,1219,1158,1132,733$. 


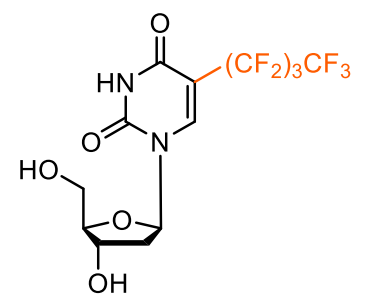

1-((2R,4S,5R)-4-Hydroxy-5-(hydroxymethyl)tetrahydrofuran-2-yl)-5(perfluorobutyl)pyrimidine-2,4(1H,3H)-dione (11): Prepared according to the general procedure from 2'-deoxyuridine $(68 \mathrm{mg}, 0.3 \mathrm{mmol})$, perfluorobutyl iodide (156 $\mu \mathrm{L}, 0.9 \mathrm{mmol}, 3$ equiv), 2,5-di-tertbutylhydroquinone $(7.0 \mathrm{mg}, 0.03 \mathrm{mmol}, 10 \mathrm{~mol} \%)$ and $\mathrm{NaHCO}_{3}(50 \mathrm{mg}$, $0.6 \mathrm{mmol}, 2$ equiv) in $2 \mathrm{~mL}$ of 11:1 MeCN:MeOH. The reaction was irradiated with two Kessil A160WE Tuna Sun LEDs for $30 \mathrm{~h}$ at $25^{\circ} \mathrm{C}$. Purified by flash column chromatography $(0-5 \%$ $\mathrm{DCM} \rightarrow$ Methanol) to give the title compound as a white solid in $48 \%$ yield (67 mg).

${ }^{1}$ H NMR: (400 MHz, CD 3 OD) $\delta 8.85(\mathrm{~s}, 1 \mathrm{H}), 6.29(\mathrm{t}, J=6.2 \mathrm{~Hz}, 1 \mathrm{H}), 4.47-4.42(\mathrm{~m}, 1 \mathrm{H}), 4.04$ $-4.00(\mathrm{~m}, 1 \mathrm{H}), 3.90-3.70(\mathrm{~m}, 2 \mathrm{H}), 3.45-3.32(\mathrm{~m}, 2 \mathrm{H}), 2.47-2.38(\mathrm{~m}, 1 \mathrm{H}), 2.33-2.25(\mathrm{~m}$, 1H). ${ }^{13}$ C NMR: $\left(201 \mathrm{MHz}, \mathrm{CD}_{3} \mathrm{OD}\right) \delta 160.9,151.2,146.3(\mathrm{t}, J=10.0 \mathrm{~Hz}), 120.0-110.4(\mathrm{~m})$, $103.3(\mathrm{t}, J=24.1 \mathrm{~Hz}), 89.4,87.7,71.8,62.1,42.3 .{ }^{19} \mathbf{F}$ NMR: $\left(376 \mathrm{MHz}, \mathrm{CD}_{3} \mathrm{OD}\right) \delta-82.37--$ 82.48 (m, 3F), -110.30 (m, 2F), -123.20 (m, 2F), -126.96 - -127.11 (m, 2F). Rf: 0.33 (8:1 DCM:MeOH) Reference: Chem. Commun. 2018, 54, 13662-13665.

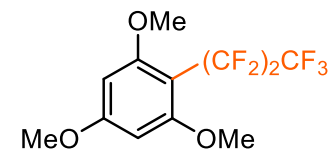

1,3,5-Trimethoxy-2-(perfluoropropyl)benzene (12): Prepared according to the general procedure from 1,3,5-trimethoxybenzene ( $58 \mathrm{mg}, 0.3 \mathrm{mmol})$, perfluoropropyl iodide (43 $\mu \mathrm{L}, \quad 0.3 \mathrm{mmol}, 1$ equiv), 2,5-di-tertbutylhydroquinone (7.0 mg, $0.03 \mathrm{mmol}, 10 \mathrm{~mol} \%$ ) and $\mathrm{NaHCO}_{3}(50 \mathrm{mg}, 0.6 \mathrm{mmol}, 2$ equiv) in 2 $\mathrm{mL}$ of 11:1 MeCN:MeOH. The reaction was irradiated with two Kessil A160WE Tuna Sun LEDs for $30 \mathrm{~h}$ at $25^{\circ} \mathrm{C}$. Purified by flash column chromatography (Hex $\rightarrow$ 9:1 Hex:EtOAc) to give the title compound as an off-white solid in $79 \%$ yield $(80 \mathrm{mg})$.

${ }^{1} \mathbf{H}$ NMR: $\left(400 \mathrm{MHz}, \mathrm{CDCl}_{3}\right) \delta 6.14$ (s, 2H), 3.84 (s, 3H), 3.80 (s, 6H). ${ }^{13} \mathbf{C}$ NMR: $(201 \mathrm{MHz}$, $\left.\mathrm{CDCl}_{3}\right) \delta 164.0,161.8,122.4-107.3(\mathrm{~m}), 98.5(\mathrm{t}, J=21.8 \mathrm{~Hz}), 91.8,56.4,55.5 .{ }^{19} \mathbf{F}$ NMR: $(376$ $\left.\mathrm{MHz}, \mathrm{CDCl}_{3}\right) \delta-80.65$ (t, $\left.J=9.3 \mathrm{~Hz}, 3 \mathrm{~F}\right),-103.44--103.60$ (m, 2F), -126.81 - -126.86 (m, 2F). Rf: 0.51 (4:1 Hex:EtOAc) Reference: Chem. Commun. 2017, 53, 10878-10881. 
(CFe

1,3,5-Trimethoxy-2-(perfluorohexyl)benzene (13): Prepared according to the general procedure from 1,3,5-trimethoxybenzene $(58 \mathrm{mg}, 0.3 \mathrm{mmol})$, perfluorohexyl iodide (194 $\mu \mathrm{L}, 0.9 \mathrm{mmol}, 3$ equiv), 2,5-di-tertbutylhydroquinone ( $7.0 \mathrm{mg}, 0.03 \mathrm{mmol}, 10 \mathrm{~mol} \%$ ) and $\mathrm{NaHCO}_{3}(50 \mathrm{mg}, 0.6 \mathrm{mmol}, 2$ equiv) in 2 $\mathrm{mL}$ of 11:1 MeCN:MeOH. The reaction was irradiated with two Kessil A160WE Tuna Sun LEDs for $30 \mathrm{~h}$ at $25^{\circ} \mathrm{C}$. Purified by flash column chromatography (Hex $\rightarrow$ 9:1 Hex:EtOAc) to give the title compound as a pale-yellow solid in quantitative yield (146 mg).

${ }^{1}$ H NMR: $\left(400 \mathrm{MHz}, \mathrm{CDCl}_{3}\right) \delta 6.15$ (s, 2H), 3.84 (s, 3H), 3.80 (s, 6H). ${ }^{13} \mathrm{C}$ NMR: $(201 \mathrm{MHz}$, $\left.\mathrm{CDCl}_{3}\right) \delta 124.3-104.3(\mathrm{~m}), 98.8(\mathrm{t}, J=21.9 \mathrm{~Hz}), 91.9,56.4,55.5 .{ }^{19} \mathbf{F} \mathbf{~ N M R}:\left(376 \mathrm{MHz}, \mathrm{CDCl}_{3}\right)$ $\delta$-80.75 - -81.02 (m), -102.56 - -102.97 (m), -120.55 - -122.44 (m), -122.69, -126.05 - -126.39 (m). Rf: 0.57 (4:1 Hex:EtOAc) Reference: Adv. Synth. Catal. 2013, 355, 2203-2207.

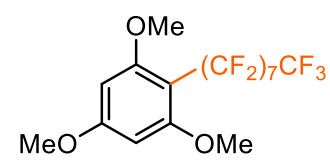

1,3,5-Trimethoxy-2-(perfluorooctyl)benzene (14): Prepared according to the general procedure from 1,3,5-trimethoxybenzene (58 $\mathrm{mg}, 0.3 \mathrm{mmol}$ ), perfluoropropyl iodide (241 $\mu \mathrm{L}, 0.9 \mathrm{mmol}, 3$ equiv), 2,5-di-tertbutylhydroquinone (7.0 mg, $0.03 \mathrm{mmol}, 10 \mathrm{~mol} \%$ ) and $\mathrm{NaHCO}_{3}(50 \mathrm{mg}, 0.6 \mathrm{mmol}, 2$ equiv) in 2 mL of 11:1 MeCN:MeOH. The reaction was irradiated with two Kessil A160WE Tuna Sun LEDs for $30 \mathrm{~h}$ at $25^{\circ} \mathrm{C}$. Purified by flash column chromatography (Hex $\rightarrow$ 9:1 Hex:EtOAc) to give the title compound as a pale-yellow solid in quantitative yield $(179 \mathrm{mg})$.

${ }^{1}$ H NMR: $\left(400 \mathrm{MHz}, \mathrm{CDCl}_{3}\right) \delta 6.15(\mathrm{~s}, 2 \mathrm{H}), 3.84(\mathrm{~s}, 3 \mathrm{H}), 3.80(\mathrm{~s}, 6 \mathrm{H}) .{ }^{13} \mathrm{C} \mathrm{NMR:}(201 \mathrm{MHz}$, $\left.\mathrm{CDCl}_{3}\right) \delta 163.0,160.9,118.5-103.7(\mathrm{~m}), 97.8(\mathrm{t}, J=21.9 \mathrm{~Hz}), 90.9,55.4,54.5 .{ }^{19} \mathbf{F}$ NMR: $(376$ $\left.\mathrm{MHz}, \mathrm{CDCl}_{3}\right) \delta-80.83(\mathrm{t}, J=10.1 \mathrm{~Hz}, 3 \mathrm{~F}),-102.50--102.98(\mathrm{~m}, 2 \mathrm{~F}),-121.49--122.20(\mathrm{~m}, 8 \mathrm{~F})$, -122.73 (s, 2F), -125.83 - -126.40 (m, 2F). Rf: 0.53 (4:1 Hex:EtOAc) Reference: Chem. Commun. 2017, 53, 10878-10881.

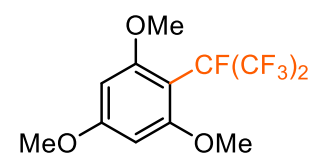

1,3,5-Trimethoxy-2-(perfluoroisopropyl)benzene (15): Prepared according to the general procedure from 1,3,5-trimethoxybenzene (58 $\mathrm{mg}, 0.3 \mathrm{mmol}$ ), perfluoroisopropyl iodide (128 $\mu \mathrm{L}, 0.9 \mathrm{mmol}, 3$ equiv), 2,5-di-tertbutylhydroquinone ( $7.0 \mathrm{mg}, 0.03 \mathrm{mmol}, 10 \mathrm{~mol} \%)$ and $\mathrm{NaHCO}_{3}(50.0 \mathrm{mg}, 0.6 \mathrm{mmol}, 2$ equiv) in $2 \mathrm{~mL}$ of 11:1 MeCN:MeOH. The reaction was irradiated with two Kessil A160WE Tuna Sun 
LEDs for $30 \mathrm{~h}$ at $25{ }^{\circ} \mathrm{C}$. Purified by flash column chromatography (Hex $\rightarrow$ 9:1 Hex:EtOAc) to give the title compound as a white solid in $34 \%$ yield $(23 \mathrm{mg})$.

${ }^{1}$ H NMR: $\left(400 \mathrm{MHz}, \mathrm{CDCl}_{3}\right) \delta 6.20-6.17(\mathrm{~m}, 1 \mathrm{H}), 6.15-6.11(\mathrm{~m}, 1 \mathrm{H}), 3.83(\mathrm{~s}, 3 \mathrm{H}), 3.81(\mathrm{~s}$, 3H), 3.78 (s, 3H). ${ }^{13}$ C NMR: (201 MHz, $\left.\mathrm{CDCl}_{3}\right) \delta$ 163.3, 162.6, $160.2(\mathrm{~d}, J=7.3 \mathrm{~Hz}), 121.6(\mathrm{qd}$, $J=288.6,29.5 \mathrm{~Hz}), 97.5(\mathrm{~d}, J=20.7 \mathrm{~Hz}), 95.9-93.5(\mathrm{~m}), 93.1,91.2,56.7,55.9,55.5 .{ }^{19}$ F NMR: $\left(376 \mathrm{MHz}, \mathrm{CDCl}_{3}\right) \delta-74.51(\mathrm{~d}, J=3.4 \mathrm{~Hz}, 6 \mathrm{~F}),-174.23--174.38(\mathrm{~m}, 1 \mathrm{~F}) . \mathbf{R}_{\mathbf{f}}: 0.51(4: 1$ Hex:EtOAc) HRMS (ESI) m/z: [M+H] ${ }^{+}$calculated for C12H11F7O3 337.0674; found 337.0676. IR (neat, $\mathbf{c m}^{-1}$ ): 3005, 2946, 2846, 1599, 1520, 1470, 1350, 1274, 1220, 1127, 1046, 935, 898, $865,770,750,731,533$.

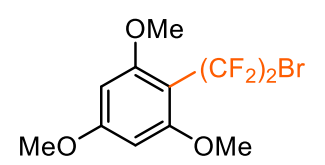

2-(2-Bromo-1,1,2,2-tetrafluoroethyl)-1,3,5-trimethoxybenzene

(16):

Prepared according to the general procedure from 1,3,5-trimethoxybenzene (58 mg, $0.3 \mathrm{mmol}$ ), 1-bromo-2-iodotetrafluoroethane (168 $\mu \mathrm{L}, 0.9 \mathrm{mmol}, 3$ equiv), 2,5-di-tert-butylhydroquinone (7.0 mg, $0.03 \mathrm{mmol}, 10 \mathrm{~mol} \%$ ) and $\mathrm{NaHCO}_{3}(50 \mathrm{mg}, 0.6$ mmol, 2 equiv) in $2 \mathrm{~mL}$ of 11:1 MeCN:MeOH. The reaction was irradiated with two Kessil A160WE Tuna Sun LEDs for $30 \mathrm{~h}$ at $25^{\circ} \mathrm{C}$. Purified by flash column chromatography (Hex $\rightarrow$ 9:1 Hex:EtOAc) to give the title compound as a white solid in $26 \%$ yield $(28 \mathrm{mg})$.

${ }^{1} \mathbf{H}$ NMR: $\left(400 \mathrm{MHz}, \mathrm{CDCl}_{3}\right) \delta 6.14(\mathrm{~s}, 2 \mathrm{H}), 3.84(\mathrm{~s}, 3 \mathrm{H}), 3.80(\mathrm{~s}, 6 \mathrm{H}) .{ }^{13} \mathbf{C}$ NMR: $(201 \mathrm{MHz}$, $\left.\mathrm{CDCl}_{3}\right) \delta 163.9,161.8,119.4(\mathrm{tt}, J=313.6,46.3 \mathrm{~Hz}), 115.8(\mathrm{tt}, J=258.5,34.7 \mathrm{~Hz}), 98.0(\mathrm{t}, J=$ 22.4 Hz), 56.5, 55.5. ${ }^{19}$ F NMR: $\left(376 \mathrm{MHz}, \mathrm{CDCl}_{3}\right) \delta-63.63(\mathrm{t}, J=5.5 \mathrm{~Hz}),-99.15(\mathrm{t}, J=5.5 \mathrm{~Hz})$. Rf: 0.42 (4:1 Hex:EtOAc) Reference: J. Fluorine Chem. 2010, 131, 746-750.

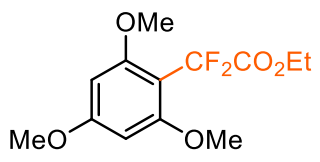

Ethyl 2,2-difluoro-2-(2,4,6-trimethoxyphenyl)acetate (17): Prepared according to the general procedure from 1,3,5-trimethoxybenzene $(58 \mathrm{mg}$, $0.3 \mathrm{mmol}$ ), ethyliodofluoroacetate ( $132 \mu \mathrm{L}, 0.9 \mathrm{mmol}, 3$ equiv), 2,5-di-tertbutylhydroquinone ( $7.0 \mathrm{mg}, 0.03 \mathrm{mmol}, 10 \mathrm{~mol} \%)$ and $\mathrm{NaHCO}_{3}(50 \mathrm{mg}, 0.6 \mathrm{mmol}, 2$ equiv) in 2 $\mathrm{mL}$ of 11:1 MeCN:MeOH. The reaction was irradiated with two Kessil A160WE Tuna Sun LEDs for $30 \mathrm{~h}$ at $25^{\circ} \mathrm{C}$. Purified by flash column chromatography (Hex $\rightarrow$ 9:1 Hex:EtOAc) to give the title compound as a white solid in $75 \%$ yield $(65 \mathrm{mg})$. 
${ }^{1}$ H NMR: $\left(400 \mathrm{MHz}, \mathrm{CDCl}_{3}\right) \delta 6.12(\mathrm{~s}, 2 \mathrm{H}), 4.32(\mathrm{q}, J=7.1 \mathrm{~Hz}, 2 \mathrm{H}), 3.82(\mathrm{~s}, 3 \mathrm{H}), 3.79(\mathrm{~s}, 6 \mathrm{H})$, $1.32(\mathrm{t}, J=7.2 \mathrm{~Hz}, 3 \mathrm{H}) .{ }^{13} \mathrm{C}$ NMR: $\left(201 \mathrm{MHz}, \mathrm{CDCl}_{3}\right) \delta 165.0(\mathrm{t}, J=33.3 \mathrm{~Hz}), 163.4,160.3$, $113.5(\mathrm{t}, J=247.7 \mathrm{~Hz}), 102.9(\mathrm{t}, J=24.1 \mathrm{~Hz}), 91.6,62.5,56.3,55.5,14.2 .{ }^{19}$ F NMR: $(376 \mathrm{MHz}$, $\left.\mathrm{CDCl}_{3}\right) \delta$-96.38 (s, 2F). Rf: 0.45 (2:1 Hex:EtOAc) Reference: Chem. Commun. 2020, 56, 18151818.

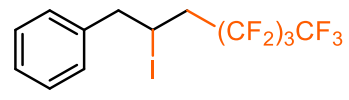

(4,4,5,5,6,6,7,7,7-Nonafluoro-2-iodoheptyl)benzene (18): Prepared according to the general procedure from allylbenzene $(40 \mu \mathrm{L}, 0.3 \mathrm{mmol})$, perfluorobutyl iodide (156 $\mu \mathrm{L}, 0.9 \mathrm{mmol}, 3$ equiv), 2,5-di-tert-butylhydroquinone $(7.0 \mathrm{mg}, 0.03$ mmol, $10 \mathrm{~mol} \%$ ) and $\mathrm{NaHCO}_{3}(50 \mathrm{mg}, 0.6 \mathrm{mmol}, 2$ equiv) in $2 \mathrm{~mL}$ of 11:1 MeCN:MeOH. The reaction was irradiated with two Kessil A160WE Tuna Sun LEDs for $30 \mathrm{~h}$ at $25{ }^{\circ} \mathrm{C}$. Purified by flash column chromatography (Hex $\rightarrow$ 9:1 Hex:EtOAc) to give the title compound as a colorless oil in $68 \%$ yield $(95 \mathrm{mg})$.

${ }^{1}$ H NMR: (400 MHz, $\left.\mathrm{CDCl}_{3}\right) \delta 7.60$ - $7.18(\mathrm{~m}, 5 \mathrm{H}), 4.51-4.41(\mathrm{~m}, 1 \mathrm{H}), 3.46-3.16(\mathrm{~m}, 2 \mathrm{H})$, 3.07 - 2.75 (m, 2H). ${ }^{13} \mathbf{C}$ NMR: $\left(201 \mathrm{MHz} \mathrm{CDCl}_{3}\right) \delta 138.7,129.4,129.1,128.8,127.5,120.5-$ $107.3(\mathrm{~m}), 47.2(\mathrm{~d}, J=1.9 \mathrm{~Hz}), 40.9(\mathrm{t}, J=20.8 \mathrm{~Hz}), 19.4 .{ }^{19}$ F NMR: $\left(376 \mathrm{MHz}, \mathrm{CDCl}_{3}\right) \delta-81.05$ (m, 3F), -110.82--114.73 (m, 2F), -124.53--124.61 (m, 2F), -125.88--125.95 (m, 2F). Rf: 0.50 (9:1 Hex:EtOAc) Reference: Chem. Commun. 2020, 56, 1815-1818.

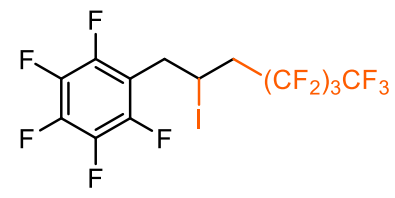

\section{1,2,3,4,5-pentafluoro-6-(4,4,5,5,6,6,7,7,7-nonafluoro-2-iodoheptyl)-} benzene (19): Prepared according to the general procedure from allylpentafluorobenzene $(46 \mu \mathrm{L}, 0.3 \mathrm{mmol})$, perfluorobutyl iodide (156 $\mu \mathrm{L}, 0.9 \mathrm{mmol}, 3$ equiv), 2,5-di-tert-butylhydroquinone (7.0 mg, $0.03 \mathrm{mmol}, 10 \mathrm{~mol} \%)$ and $\mathrm{NaHCO}_{3}(50 \mathrm{mg}, 0.6 \mathrm{mmol}, 2$ equiv) in $2 \mathrm{~mL}$ of $11: 1 \mathrm{MeCN}: \mathrm{MeOH}$. The reaction was irradiated with two Kessil A160WE Tuna Sun LEDs for $30 \mathrm{~h}$ at $25{ }^{\circ} \mathrm{C}$. Purified by flash column chromatography (Hex $\rightarrow$ 9:1 Hex:EtOAc) to give the title compound as a colorless oil in $61 \%$ yield (102 mg).

${ }^{1}$ H NMR: (400 MHz, $\left.\mathrm{CDCl}_{3}\right) \delta 4.56-4.46(\mathrm{~m}, 1 \mathrm{H}), 3.45-3.26(\mathrm{~m}, 2 \mathrm{H}), 3.13-2.78(\mathrm{~m}, 2 \mathrm{H})$. ${ }^{13}$ C NMR: (201 MHz, $\left.\mathrm{CDCl}_{3}\right) \delta 145.0$ - $143.6(\mathrm{~m}), 140.6$ - 139.1, 137.5 - 136.1 (m), 118.8 $114.1(\mathrm{~m}), 112.1(\mathrm{td}, J=17.8,3.9 \mathrm{~Hz}), 110.7-106.2(\mathrm{~m}), 41.0(\mathrm{t}, J=21.1 \mathrm{~Hz}), 32.9,13.6 .{ }^{19} \mathbf{F}$ 
NMR: $\left(376 \mathrm{MHz}, \mathrm{CDCl}_{3}\right) \delta-80.95--81.05(\mathrm{~m}, 3 \mathrm{~F}),-110.88--115.14(\mathrm{~m}, 2 \mathrm{~F}),-124.42(\mathrm{t}, J=$ $5.1 \mathrm{~Hz}, 2 \mathrm{~F}),-125.80--125.95(\mathrm{~m}, 2 \mathrm{~F}),-142.07--142.22(\mathrm{~m}, 2 \mathrm{~F}),-154.38(\mathrm{t}, J=20.8 \mathrm{~Hz}, 1 \mathrm{~F})$, 161.41 - -161.63 (m, 2F). Rf: 0.50 (Hexane) HRMS (GC CI) m/z: [M-I] $]^{+}$calculated for $\mathrm{C}_{13} \mathrm{H}_{5} \mathrm{~F}_{14} \mathrm{I}$ 427.0168; found 427.0180. IR (neat, $\mathbf{c m}^{-1}$ ): 2926, 2854, 1658, 1522, 1350, 1221, 1132, 979, 970, $881,738,721,514$.

BocHN $\left.ح \mathrm{CF}_{2}\right)_{3} \mathrm{CF}_{3}$ Tert-butyl (4,4,5,5,6,6,7,7,7-nonafluoro-2-iodoheptyl)carbamate (20):

Prepared according to the general procedure from tert-butyl- $N$ allylcarbamate (47 mg, $0.3 \mathrm{mmol}$ ), perfluorobutyl iodide (156 $\mu \mathrm{L}, 0.9 \mathrm{mmol}, 3$ equiv), 2,5-di-tertbutylhydroquinone (7.0 mg, $0.03 \mathrm{mmol}, 10 \mathrm{~mol} \%$ ) and $\mathrm{NaHCO}_{3}(50 \mathrm{mg}, 0.6 \mathrm{mmol}, 2$ equiv) in 2 mL of 11:1 MeCN:MeOH. The reaction was irradiated with two Kessil A160WE Tuna Sun LEDs for $30 \mathrm{~h}$ at $25^{\circ} \mathrm{C}$. Purified by flash column chromatography (Hex $\rightarrow$ 9:1 Hex:EtOAc) to give the title compound as a white solid in $67 \%$ yield $(80 \mathrm{mg})$.

${ }^{1}$ H NMR: (400 MHz, $\left.\mathrm{CDCl}_{3}\right) \delta 4.97$ (b.s, 1H), 4.38 (p, J = 6.6 Hz, 1H), $3.64-3.40$ (m, 2H), 2.92 - 2.67 (m, 2H), 1.45 (s, 9H). ${ }^{13}$ C NMR: (101 MHz, $\left.\mathrm{CDCl}_{3}\right) \delta$ 155.7, 120.2 - 114.7 (m), 80.4, 49.1, $38.7(\mathrm{t}, J=21.3 \mathrm{~Hz}), 29.9,28.4,18.8 .{ }^{19} \mathbf{F}$ NMR: $\left(376 \mathrm{MHz}, \mathrm{CDCl}_{3}\right) \delta-81.02(\mathrm{tt}, J=9.6,3.3 \mathrm{~Hz}$, 3F), $-112.34--114.53(\mathrm{~m}, 2 \mathrm{~F}),-124.58(\mathrm{~m}, 2 \mathrm{~F}),-125.83--125.93$ (m, 2F). Rf: 0.32 (9:1 Hex:EtOAc) Reference: Chem. Commun. 2020, 56, 1815-1818.

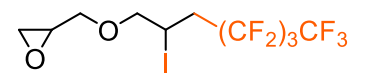

2-((4,4,5,5,6,6,7,7,7-nonafluoro-2-iodoheptyl)oxy)methyl)oxirane (21):

Prepared according to the general procedure from allyl glycidyl ether (34 $\mu \mathrm{L}, 0.3 \mathrm{mmol})$, perfluorobutyl iodide (156 $\mu \mathrm{L}, 0.9 \mathrm{mmol}, 3$ equiv), 2,5-di-tert-butylhydroquinone (7.0 mg, $0.03 \mathrm{mmol}, 10 \mathrm{~mol} \%$ ) and $\mathrm{NaHCO}_{3}(50 \mathrm{mg}, 0.6 \mathrm{mmol}, 2$ equiv) in $2 \mathrm{~mL}$ of 11:1 MeCN:MeOH. The reaction was irradiated with two Kessil A160WE Tuna Sun LEDs for $30 \mathrm{~h}$ at $25^{\circ} \mathrm{C}$. Purified by flash column chromatography (Hex $\rightarrow$ 9:1 Hex:EtOAc) to give the title compound as a colorless oil in $35 \%$ yield $(48 \mathrm{mg})$.

${ }^{1}$ H NMR: (400 MHz, $\left.\mathrm{CDCl}_{3}\right) \delta 4.41$ - $4.32(\mathrm{~m}, 1 \mathrm{H}), 3.91-3.67(\mathrm{~m}, 3 \mathrm{H}), 3.45$ (dd, $J=11.9,6.0$ $\mathrm{Hz}, 1 \mathrm{H}), 3.21-3.12(\mathrm{~m}, 1 \mathrm{H}), 3.14-2.96(\mathrm{~m}, 1 \mathrm{H}), 2.84-2.80(\mathrm{~m}, 1 \mathrm{H}), 2.78-2.59(\mathrm{~m}, 2 \mathrm{H}) .{ }^{13} \mathrm{C}$ NMR: (201 MHz, $\left.\mathrm{CDCl}_{3}\right) \delta 207.1,120.3-107.6(\mathrm{~m}), 76.1(\mathrm{~d}, J=13.7 \mathrm{~Hz}), 71.8(\mathrm{~d}, J=14.0 \mathrm{~Hz})$, $50.8(\mathrm{~d}, J=4.9 \mathrm{~Hz}), 44.1(\mathrm{~d}, J=6.8 \mathrm{~Hz}), 37.6(\mathrm{t}, J=21.0 \mathrm{~Hz}), 31.1,14.3(\mathrm{~d}, J=20.9 \mathrm{~Hz}) .{ }^{\mathbf{1 9}} \mathbf{F}$ 
NMR: $\left(376 \mathrm{MHz}, \mathrm{CDCl}_{3}\right) \delta-80.95--81.07$ (m, 3F), -112.71 - -114.87 (m, 2F), -124.41 - -124.68 (m, 2F), -125.75 - -126.05 (m, 2F). Rf: 0.20 (9:1 Hex:EtoAc) Reference: Russ. Chem. Bull., Int. Ed. 2007, 56, 1534-1536.

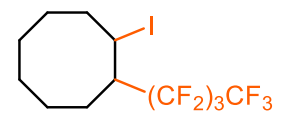

1-Iodo-2-(perfluorobutyl)cyclooctane (22): Prepared according to the general from cyclooctene $(39 \mu \mathrm{L}, 0.3 \mathrm{mmol})$, perfluorobutyl iodide $(156 \mu \mathrm{L}, 0.9 \mathrm{mmol}$, 3 equiv), 2,5-di-tert-butylhydroquinone $(7.0 \mathrm{mg}, 0.03 \mathrm{mmol}, 10 \mathrm{~mol} \%)$ and $\mathrm{NaHCO}_{3}(50 \mathrm{mg}, 0.6$ mmol, 2 equiv) in $2 \mathrm{~mL}$ of 11:1 MeCN:MeOH. The reaction was irradiated with two Kessil A160WE Tuna Sun LEDs for $30 \mathrm{~h}$ at $25^{\circ} \mathrm{C}$. Purified by flash column chromatography (Hex $\rightarrow$ 9:1 Hex:EtOAc) to give the title compound in a mixture of diastereomers as a colorless oil in $70 \%$ yield (96 mg, 1.6:1 dr).

${ }^{1}$ H NMR: $\left(400 \mathrm{MHz}, \mathrm{CDCl}_{3}\right) \delta 4.64-4.58(\mathrm{~m}, 1 \mathrm{H}$, major diastereomer), $4.56-4.49(\mathrm{~m}, 1 \mathrm{H}$, minor diastereomer), $2.50-2.3(\mathrm{~m}, 3 \mathrm{H}), 2.17-1.38(\mathrm{~m}, 10 \mathrm{H}) .{ }^{13} \mathbf{C} \mathbf{N M R}:\left(201 \mathrm{MHz}, \mathrm{CDCl}_{3}\right.$, mixture of diastereomers) $\delta 123.6-105.5(\mathrm{~m}), 40.9(\mathrm{t}, J=20.2 \mathrm{~Hz}$, diastereomer 1$), 40.5(\mathrm{t}, J=$ $20.2 \mathrm{~Hz}$, diastereomer 2), 39.7, 38.8, 38.3, 36.1, 35.0 (CHI, diastereomer 1), 34.8 (CHI, diastereomer 2), 27.8, 27.3, 26.7, 25.5, 25.0, 23.7. ${ }^{19}$ F NMR: $\left(376 \mathrm{MHz}, \mathrm{CDCl}_{3}\right) \delta-80.84--81.19$ (m, 3F), -107.86--118.27 (m, 2F), -119.84--123.60 (m, 2F), -124.41 - -127.33 (m, 2F). Rf: 0.84 (9:1 Hex:EtOAc) Reference: Org. Lett. 2019, 21, 7823-7827.

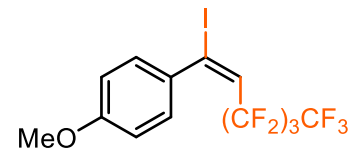

(E)-1-methoxy-4-(3,3,4,4,5,5,6,6,6-nonafluoro-1-en-1-yl)benzene $\quad(23)$ : Prepared according to the general procedure from allyl glycidyl ether (34 $\mu \mathrm{L}, 0.3 \mathrm{mmol}$ ), perfluorobutyl iodide ( $156 \mu \mathrm{L}, 0.9 \mathrm{mmol}, 3$ equiv), 2,5-ditert-butylhydroquinone (7.0 mg, $0.03 \mathrm{mmol}, 10 \mathrm{~mol} \%$ ) and $\mathrm{NaHCO}_{3}(50 \mathrm{mg}, 0.6 \mathrm{mmol}, 2$ equiv) in $2 \mathrm{~mL}$ of 11:1 MeCN:MeOH. The reaction was irradiated with two Kessil A160WE Tuna Sun LEDs for $30 \mathrm{~h}$ at $25^{\circ} \mathrm{C}$. Purified by flash column chromatography (Hex $\rightarrow$ 9:1 Hex:EtOAc) to give the title compound as a colorless oil in 66\% yield (95 mg, $E: Z=2.8: 1$ ).

${ }^{1}$ H NMR: (400 MHz, $\mathrm{CDCl}_{3}, E$ isomer) $\delta 7.30-7.23(\mathrm{~m}, 2 \mathrm{H}), 6.90-6.80(\mathrm{~m}, 2 \mathrm{H}), 6.55(\mathrm{t}, J=$ $13.4 \mathrm{~Hz}, 1 \mathrm{H}), 3.82$ (s, 3H). ${ }^{13} \mathrm{C}$ NMR: $\left(201 \mathrm{MHz}, \mathrm{CDCl}_{3}, E\right.$ isomer) $\delta 160.5,133.9,129.1,126.5$ $(\mathrm{t}, J=21.8 \mathrm{~Hz}), 118.6-108.6(\mathrm{~m}), 113.6,55.5 .{ }^{19} \mathbf{F ~ N M R : ~}\left(376 \mathrm{MHz}, \mathrm{CDCl}_{3}, E\right.$ Isomer $) \delta-80.93$ 
- -81.14 (m, 3F), -104.66--108.87 (m, 2F), -122.12--123.98 (m, 2F), -125.22- -126.43 (m, 2F). Rf: 0.93 (9:1 Hex:EtOAc) Reference: Synlett 2021, 53, 123.

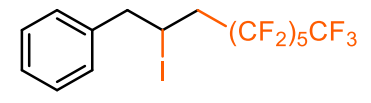

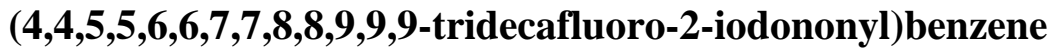

(24):

Prepared according to the general procedure from allylbenzene $(40 \mu \mathrm{L}, 0.3$ mmol), perfluorohexyl iodide (194 $\mu \mathrm{L}, 0.9 \mathrm{mmol}, 3$ equiv), 2,5-di-tert-butylhydroquinone (7.0 $\mathrm{mg}, 0.03 \mathrm{mmol}, 10 \mathrm{~mol} \%$ ) and $\mathrm{NaHCO}_{3}(50 \mathrm{mg}, 0.6 \mathrm{mmol}, 2$ equiv) in $2 \mathrm{~mL}$ of 11:1 $\mathrm{MeCN}: \mathrm{MeOH}$. The reaction was irradiated with two Kessil A160WE Tuna Sun LEDs for $30 \mathrm{~h}$ at $25{ }^{\circ} \mathrm{C}$. Purified by flash column chromatography (Hex $\rightarrow$ 9:1 Hex:EtOAc) to give the title compound as a colorless oil in $70 \%$ yield $(129 \mathrm{mg})$.

${ }^{1}$ H NMR: $\left(400 \mathrm{MHz}, \mathrm{CDCl}_{3}\right) \delta 7.59-7.18(\mathrm{~m}, 5 \mathrm{H}), 4.51-4.41(\mathrm{~m}, 1 \mathrm{H}), 3.44-3.16(\mathrm{~m}, 2 \mathrm{H})$, $3.05-2.75$ (m, 2H). ${ }^{13} \mathrm{C}$ NMR: $\left(201 \mathrm{MHz}, \mathrm{CDCl}_{3}\right) \delta 138.7,129.4,129.1,128.8,127.5,120.2-$ $106.8(\mathrm{~m}), 47.2,41.0(\mathrm{t}, J=20.8 \mathrm{~Hz}), 19.5 .{ }^{19} \mathbf{F}$ NMR: $\left(376 \mathrm{MHz}, \mathrm{CDCl}_{3}\right) \delta-80.71--80.96(\mathrm{~m}$, 3F), -110.55 - -114.85 (m, 2F), -121.31 - -122.14 (m, 2F), -122.55 - -123.15 (m, 2F), -123.21 - 124.15 (m, 2F), -125.97 - -126.46 (m, 2F). Rf: 0.50 (Hexane) Reference: Eur. J. Org. Chem. 2017, 2126-2129.

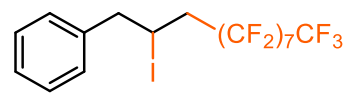

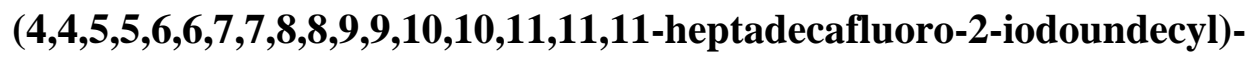
benzene (25): Prepared according to the general procedure from allylbenzene ( $40 \mu \mathrm{L}, 0.3 \mathrm{mmol}$ ), perfluorooctyl iodide (196 $\mu \mathrm{L}, 0.9 \mathrm{mmol}, 3$ equiv), 2,5-di-tertbutylhydroquinone (7.0 mg, $0.03 \mathrm{mmol}, 10 \mathrm{~mol} \%$ ) and $\mathrm{NaHCO}_{3}(50 \mathrm{mg}, 0.6 \mathrm{mmol}, 2$ equiv) in 2 $\mathrm{mL}$ of 11:1 MeCN:MeOH. The reaction was irradiated with two Kessil A160WE Tuna Sun LEDs for $30 \mathrm{~h}$ at $25^{\circ} \mathrm{C}$. Purified by flash column chromatography (Hex $\rightarrow$ 9:1 Hex:EtOAc) to give the title compound as a white solid in $79 \%$ yield $(158 \mathrm{mg})$.

${ }^{1}$ H NMR: $\left(400 \mathrm{MHz}, \mathrm{CDCl}_{3}\right) \delta 7.60-7.18(\mathrm{~m}, 5 \mathrm{H}), 4.52-4.41(\mathrm{~m}, 1 \mathrm{H}), 3.44-3.16(\mathrm{~m}, 2 \mathrm{H})$, $3.02-2.72(\mathrm{~m}, 2 \mathrm{H}) .{ }^{13} \mathrm{C}$ NMR: $\left(201 \mathrm{MHz}, \mathrm{CDCl}_{3}\right) \delta 138.7,129.1,128.8,127.5,120.2-108.0$ (m), 47.2, $41.0(\mathrm{t}, J=20.8 \mathrm{~Hz}), 19.5 .{ }^{19} \mathrm{~F}$ NMR: $\left(376 \mathrm{MHz}, \mathrm{CDCl}_{3}\right) \delta-80.77--80.94(\mathrm{~m}, 3 \mathrm{~F}),-$ 109.21 - -115.86 (m, 2F), -121.16--122.22 (m, 6F), -122.54- -123.04 (m, 2F), -123.50- -123.82 (m, 2F), -126.18 (s, 2F). Rf: 0.50 (Hexane) HRMS (GC CI) m/z: [M-I] $]^{+}$calculated for $\mathrm{C}_{17} \mathrm{H}_{10} \mathrm{~F}_{17} \mathrm{I}$ 
537.0511; found 537.0497. IR (neat, $\mathbf{c m}^{-1}$ ): 2921, 2856, 1498, 1456, 1238, 1146, 1135, 1117, 745, $722,658,504$.

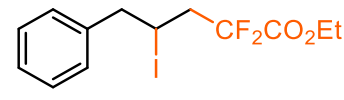

Ethyl 2,2-difluoro-4-iodo-5-phenylpentanoate (26): Prepared according to the general procedure from allylbenzene $(40 \mu \mathrm{L}, 0.3 \mathrm{mmol})$, ethyliododifluoroacetate ( $132 \mu \mathrm{L}, 0.9 \mathrm{mmol}, 3$ equiv), 2,5-di-tert-butylhydroquinone ( $7.0 \mathrm{mg}, 0.03$ mmol, $10 \mathrm{~mol} \%$ ) and $\mathrm{NaHCO}_{3}(50 \mathrm{mg}, 0.6 \mathrm{mmol}, 2$ equiv) in $2 \mathrm{~mL}$ of 11:1 MeCN:MeOH. The reaction was irradiated with two Kessil A160WE Tuna Sun LEDs for $30 \mathrm{~h}$ at $25^{\circ} \mathrm{C}$. Purified by flash column chromatography (Hex $\rightarrow$ 9:1 Hex:EtOAc) to give the title compound as a colorless oil in $62 \%$ yield $(68 \mathrm{mg})$.

${ }^{1} \mathbf{H}$ NMR: $\left(400 \mathrm{MHz}, \mathrm{CDCl}_{3}\right) \delta 7.37-7.27(\mathrm{~m}, 3 \mathrm{H}), 7.22-7.17(\mathrm{~m}, 2 \mathrm{H}), 4.40-4.30(\mathrm{~m}, 3 \mathrm{H})$, $3.30-3.16(\mathrm{~m}, 2 \mathrm{H}), 2.95-2.64(\mathrm{~m}, 2 \mathrm{H}), 1.30(\mathrm{t}, J=7.2 \mathrm{~Hz}, 3 \mathrm{H}) \cdot{ }^{13} \mathbf{C} \mathbf{N M R}:\left(101 \mathrm{MHz}, \mathrm{CDCl}_{3}\right)$ $\delta 163.6(\mathrm{t}, J=32.16 \mathrm{~Hz}), 139.0,129.1,128.7,127.4,117.9-112.8(\mathrm{~m}), 63.4,47.3,47.3,44.7$, $44.5(\mathrm{t}, J=23.50 \mathrm{~Hz}), 22.0(\mathrm{t}, J=3.75 \mathrm{~Hz}), 14.0 .{ }^{19} \mathrm{~F}$ NMR: $\left(376 \mathrm{MHz}, \mathrm{CDCl}_{3}\right) \delta-101.76(\mathrm{ddd}, J$ = 263.1, 16.8, 13.2 Hz), -106.39 (dt, $J=263.6,16.9 \mathrm{~Hz})$. Rf: 0.47 (9:1 Hex:EtOAc) Reference: Chem. Commun. 2020, 56, 1815-1818. 


\section{G. Procedure for 1 mmol Scale Radical Perfluoroalkylation Reaction}

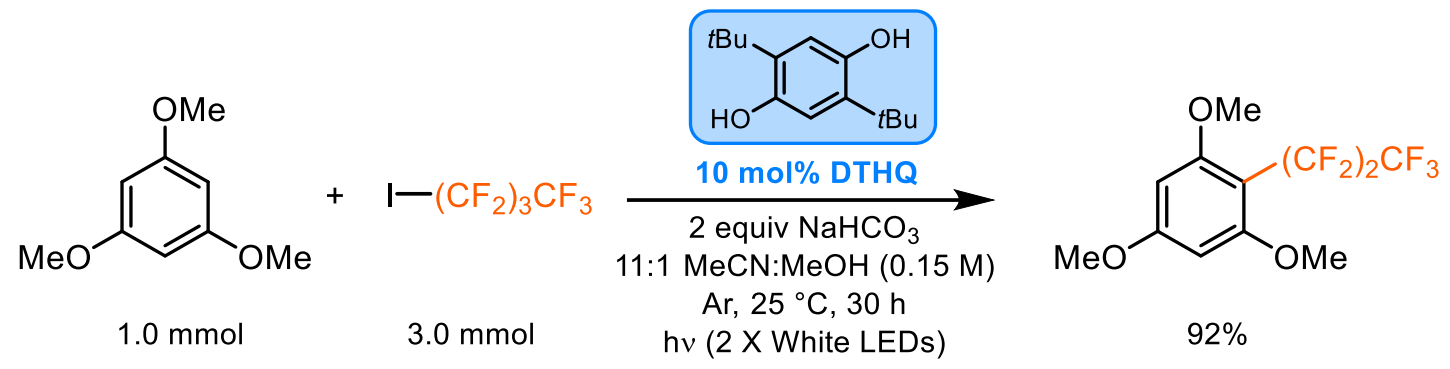

An oven-dried $25 \mathrm{~mL}$ round bottom flask equipped with a magnetic stir bar was charged with a 1,3,5-trimethoxybenzene (168 mg, $1.0 \mathrm{mmol}, 1$ equiv), perfluorobutyl iodide (1.04 mL, $3.0 \mathrm{mmol}$, 3 equiv), 2,5-di-tert-butylhydroquinone (22.2 mg, $0.1 \mathrm{mmol}, 10 \mathrm{~mol} \%$ ) and $\mathrm{NaHCO}_{3}(168 \mathrm{mg}, 2.0$ mmol, 2 equiv). 11:1 MeCN:MeOH was added $(6.7 \mathrm{~mL}, 0.15 \mathrm{M})$, and the reaction mixture was degassed by sparging with argon for 5-6 minutes. The reaction mixture was then sonicated and irradiated with two Kessil A160WE Tuna Sun LED lamps for $30 \mathrm{~h}$ at $25{ }^{\circ} \mathrm{C}$. The reaction mixture was transferred into a separatory funnel and diluted with $20 \mathrm{~mL}$ of DCM and washed with $20 \mathrm{~mL}$ of $10 \mathrm{mM} \mathrm{Na} \mathrm{S}_{2} \mathrm{O}_{3}$ (aq). The aqueous phase was extracted with $20 \mathrm{~mL}$ of DCM. The combined organic phases were dried with $\mathrm{MgSO}_{4}$ and concentrated. Purified by flash column chromatography $(0 \rightarrow 15 \%$ EtOAc in Hex) to give 1,3,5-trimethoxy-2-(perfluorobutyl)benzene as a white solid in $92 \%$ yield $(357 \mathrm{mg})$. Spectral data matched that previously reported. 


\section{H. Procedure for Radical Perfluoroalkylation Reaction with $\mathrm{C}_{4} \mathrm{~F} 9 \mathrm{Br}$}

An oven-dried two-dram borosilicate glass vial equipped with a magnetic stir bar was charged with a 1,3,5-trimethoxybenzene ( $58 \mathrm{mg}, 0.3 \mathrm{mmol})$, perfluorobutyl bromide (168 $\mu \mathrm{L}, 0.9 \mathrm{mmol}, 3$ equiv), 2,5-di-tert-butylhydroquinone ( $7.0 \mathrm{mg}, 0.03 \mathrm{mmol}, 10 \mathrm{~mol} \%$ ) and $\mathrm{NaHCO}_{3}(50.0 \mathrm{mg}, 0.6$ mmol, 2 equiv). 11:1 MeCN:MeOH was added $(2 \mathrm{~mL}, 0.15 \mathrm{M})$, and the reaction mixture was degassed by sparging with argon for 5-6 minutes. The reaction mixture was then sonicated and irradiated with two Kessil A160WE Tuna Sun LED lamps for $30 \mathrm{~h}$ at $25{ }^{\circ} \mathrm{C}$. The reaction mixture was transferred into a separatory funnel and diluted with $15 \mathrm{~mL}$ of DCM and washed with $15 \mathrm{~mL}$ of $10 \mathrm{mM} \mathrm{Na}_{2} \mathrm{~S}_{2} \mathrm{O}_{3}$ (aq). The aqueous phase was extracted with $15 \mathrm{~mL}$ of DCM. The combined organic phases were dried with $\mathrm{MgSO}_{4}$ and concentrated. Purified by flash column chromatography (Hex $\rightarrow$ 9:1 Hex:EtOAc) to give 2-(2-bromo-1,1,2,2-tetrafluoroethyl)-1,3,5trimethoxybenzene as a white solid in $26 \%$ yield $(28 \mathrm{mg})$. Spectral data matched that previously reported. 


\section{UV-Vis Spectra of 2,5-Di-tert-butylhydroquinone}

In order to determine whether the catalyst, 2,5-di-tert-butylhydroquinone (DTHQ), was deprotonated under our initial reaction conditions, we performed a series of UV-Vis measurements. No appreciable difference was observed between the absorption spectra of DTHQ and DTHQ in the presence of $\mathrm{NaHCO}_{3}$ (see Figure S3). However, in the presence of a stronger base, $\mathrm{K}_{2} \mathrm{CO}_{3}$, a significant change in the absorption spectrum compared to DTHQ was observed. This significant redshift in the absorption spectrum is characteristic of a phenolate anion. ${ }^{1-3}$ Therefore, since this change was not observed in the presence of $\mathrm{NaHCO}_{3}$, we can conclude that the charge-transfer complex observed between DTHQ and $\mathrm{C}_{4} \mathrm{~F}_{9} \mathrm{I}$ occurs with the neutral form of DTHQ, and not with the corresponding phenolate anion.

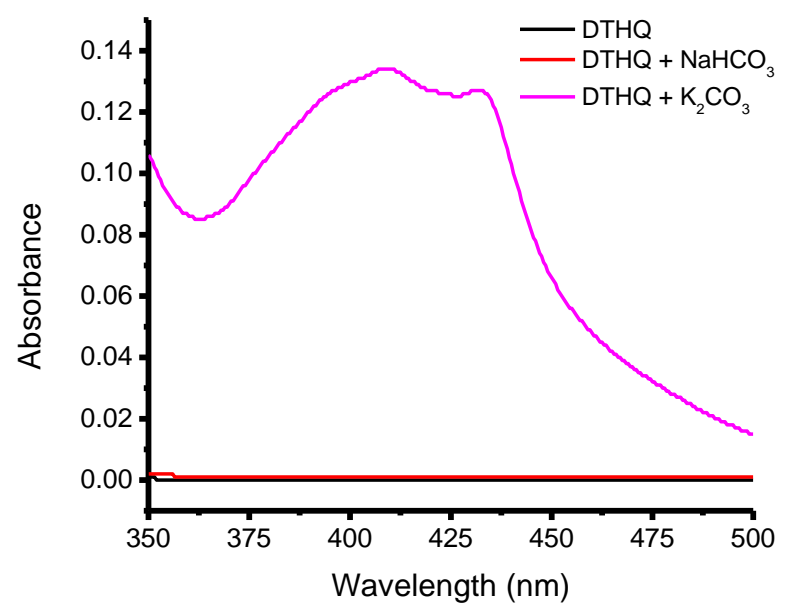

Figure S3. Absoprtion spectra of 2,5-di-tert-butylhydroquinone (DTHQ), DTHQ + $\mathrm{NaHCO}_{3}$, and $\mathrm{DTHQ}+\mathrm{K}_{2} \mathrm{CO}_{3}$. $[\mathrm{DTHQ}]=15 \mathrm{mM},\left[\mathrm{NaHCO}_{3}\right]=0.3 \mathrm{M},\left[\mathrm{K}_{2} \mathrm{CO}_{3}\right]=0.3 \mathrm{M}$ in 11:1 $\mathrm{MeCN}: \mathrm{MeOH}$. 


\section{J. Benesi-Hildebrand Analysis of Charge-Transfer Complex}

The association constant $\left(K_{a}\right)$ of the charge-transfer complex formed between 2,5-di-tertbutylhydroquinone (DTHQ) and $\mathrm{C}_{4} \mathrm{~F}_{9} \mathrm{I}$ was determined using the Benesi-Hildebrand method. ${ }^{4}$ In a quartz cuvette, the absorbance from 400-450 nm of a freshly prepared solution of DTHQ (15 $\mathrm{mM}$ ) in 11:1 MeCN:MeOH was measured. To the solution of 2,5-di-tert-butylhydroquinone was then added 5 equiv of $\mathrm{C}_{4} \mathrm{~F}_{9} \mathrm{I}$. The absorbance of this solution was then measured, and the procedure was repeated to give a range of 5 to 30 equivalents of $\mathrm{C}_{4} \mathrm{~F}_{9} \mathrm{I}$ compared to the concentration of DTHQ (see Figure S4).

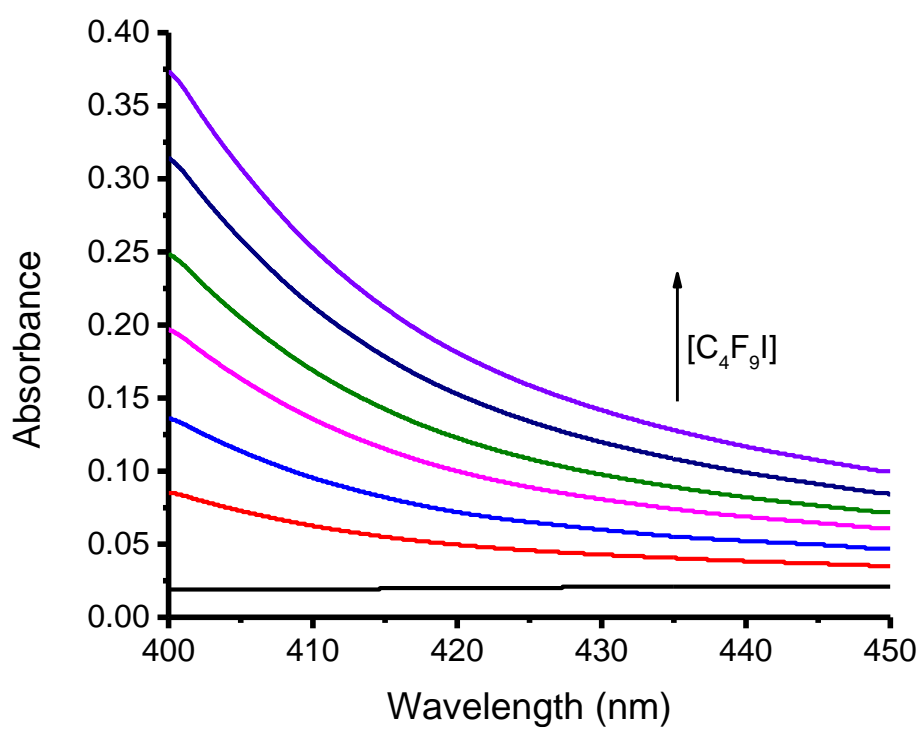

Figure S4. Absorption spectra of 2,5-di-tert-butylhydroquinone (DTHQ, $15 \mathrm{mM}$ ) with increasing concentrations of $\mathrm{C}_{4} \mathrm{~F}_{9} \mathrm{I}$ (0-30 equiv) in 11:1 $\mathrm{MeCN}: \mathrm{MeOH}$.

In order to analyze the data using the Benesi-Hildebrand method, we then plotted the reciprocal of the change of absorbance of the charge-transfer complex at $440 \mathrm{~nm}$ $\left(\triangle \mathrm{A} 440 \mathrm{~nm}\right.$ ) versus the reciprocal of the concentration of $\mathrm{C}_{4} \mathrm{~F}_{9} \mathrm{I}$. The corresponding plot can be found below in Figure S5. The linear relationship observed indicates that the stoichiometry of the DTHQ- $\mathrm{C}_{4} \mathrm{~F}_{9} \mathrm{I}$ complex is 1:1. 


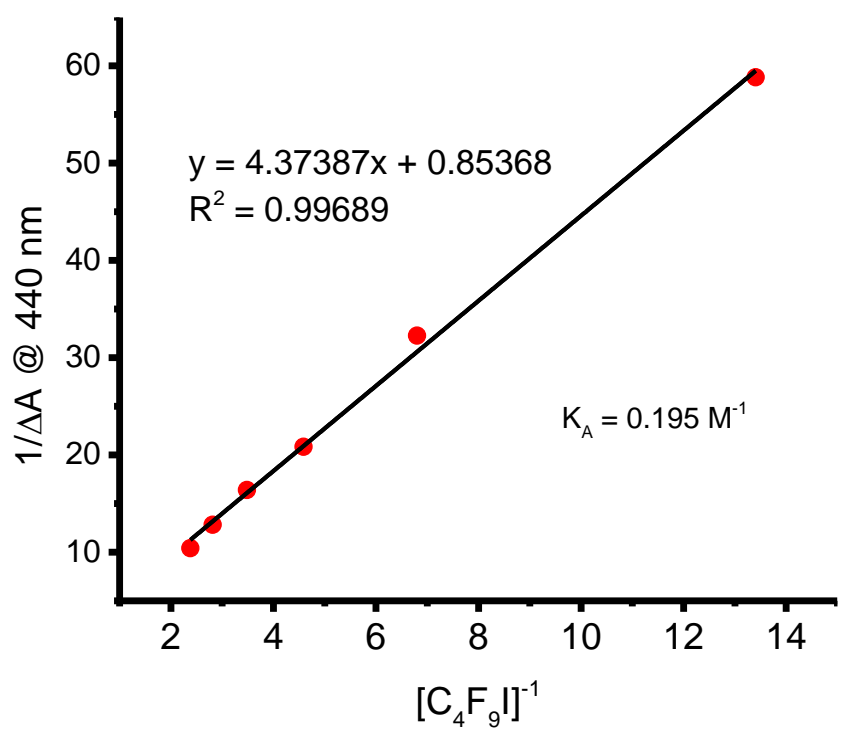

Figure S5. Benesi-Hildebrand plot for the determination of the association constant $\left(K_{a}\right)$ between 2,5-di-tert-butylhydroquinone (DTHQ) and $\mathrm{C}_{4} \mathrm{~F}_{9} \mathrm{I}$. The change in absorbance at $440 \mathrm{~nm}$ was monitored as increasing concentrations of $\mathrm{C}_{4} \mathrm{~F}_{9} \mathrm{I}$ (5-30 equiv) was added to a solution of DTHQ $(15 \mathrm{mM})$ in 11:1 MeCN:MeOH.

The linear relationship was then used to solve the Benesi-Hildebrand equation (eq S1-S4), giving a $K_{a}$ of $0.195 \mathrm{M}^{-1}$ in $11: 1 \mathrm{MeCN}: \mathrm{MeOH}$.

$$
\begin{gathered}
\frac{1}{\Delta A_{440 \mathrm{~nm}}}=\frac{1}{A_{440 \mathrm{~nm}}} \times \frac{1}{K_{a}} \frac{1}{\left[C_{4} F_{9} I\right]}+\frac{1}{A_{440 \mathrm{~nm}}} \\
\frac{1}{A_{440 \mathrm{~nm}}}=0.85368 \quad(S 2) \\
0.85368 \times \frac{1}{K_{a}}=4.37387 \quad(S 3) \\
K_{a}=0.195 \mathrm{M}^{-1} \quad(S 4)
\end{gathered}
$$


K. ${ }^{1} \mathrm{H}$ NMR Spectra of 2,5-Di-tert-butylhydroquinone

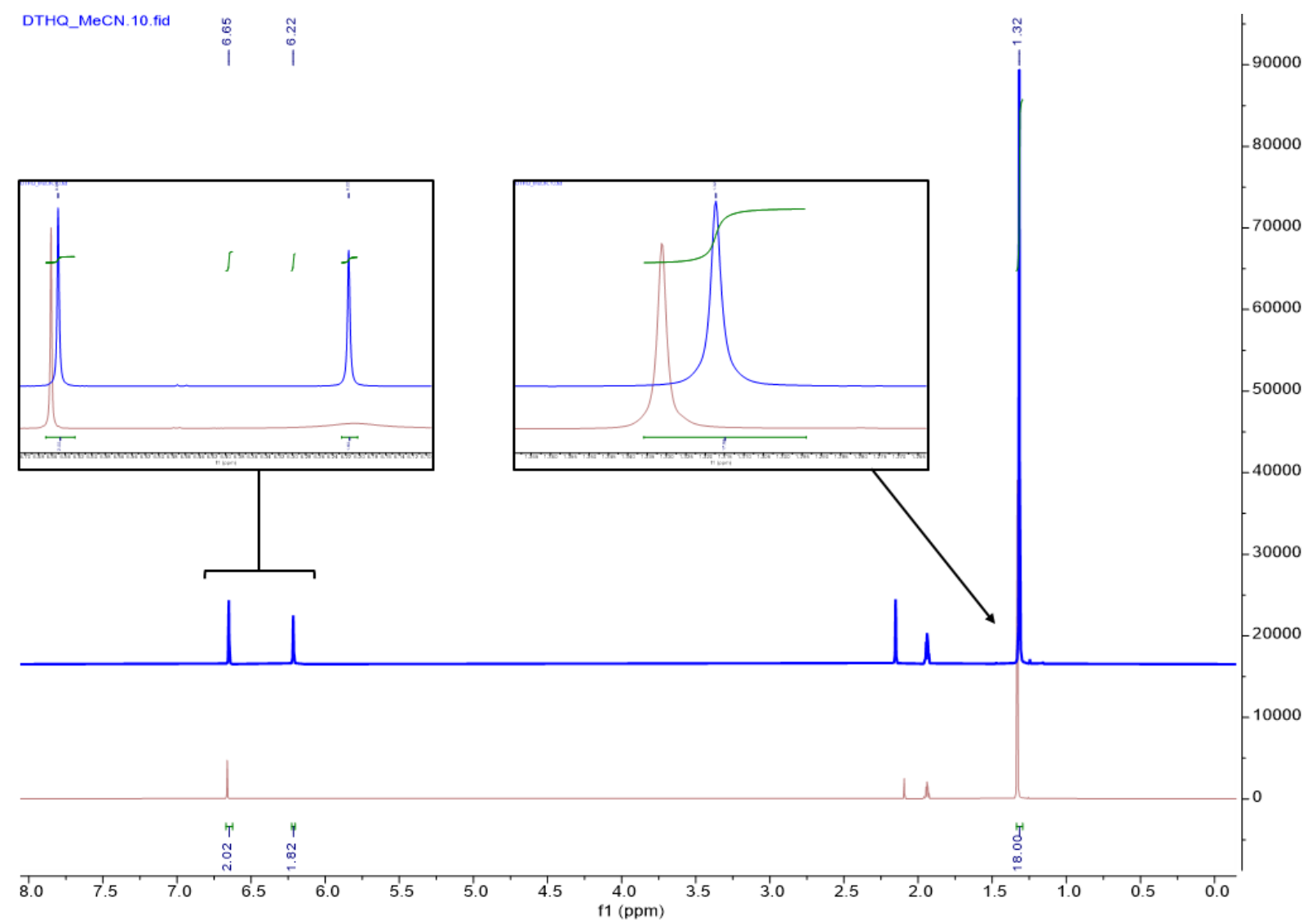

Figure S6. ${ }^{1} \mathrm{H}$ NMR spectra of DTHQ (blue, top) and DTHQ $+\mathrm{C}_{4} \mathrm{~F}_{9} \mathrm{I}$ (maroon, bottom) in $\mathrm{CD}_{3} \mathrm{CN}$. DTHQ: $0.015 \mathrm{mmol} ; \mathrm{C}_{4} \mathrm{~F}_{9} \mathrm{I}: 0.45 \mathrm{mmol} ; \mathrm{CD}_{3} \mathrm{CN}: 0.5 \mathrm{~mL}$. 


\section{Computational Studies}

The propensity for halogen bonding between ditertbutyl hydroquinone (DTHQ) and perfluoroalkane substituents was investigated computationally by assessing the net noncovalent interaction strength using density functional theory calculations. The primary aims were to both determine if halogen bonding was possible in a manner suspected in the initial experiments and determine the optimal computational approach for quantitative assessment of halogen bonding interaction in these and related potential catalysts and halogen containing molecules.

Calculation of halogen bond strength followed the procedure outlined by Šponer et al. whereby the principle non-covalent force is evaluated as the net overall interaction enthalpy, $\Delta H_{\mathrm{A}-\mathrm{B} \text {, }}^{\circ}$ between two molecules. ${ }^{5}$ The net overall interaction enthalpy is given by:

$$
\Delta H_{\mathrm{A}-\mathrm{B}}^{\circ}=H_{\mathrm{A}-\mathrm{B}}^{\circ}-\left(H_{\mathrm{A}}^{\circ}+H_{\mathrm{B}}^{\circ}\right)+H_{\mathrm{A}, \text { strain }}^{\circ}+H_{\mathrm{B}, \text { strain }}^{\circ}
$$

Here, the $H_{\mathrm{x}}^{\circ}$ values are the calculated absolute standard state enthalpies of the relevant gas phase geometry optimized structures, both the optimized complex and single molecule structures. The $H_{\mathrm{x} \text {,strain }}^{\circ}$ alues are the strain/deformation enthalpies incurred by each molecule when distorted in the complex from their optimized independent geometries. This strain enthalpy is generally a positive quantity calculated as the difference between the enthalpy of the deformed geometry and the optimized geometry. This is a substantial term in complexation energies of molecules with larger amounts of conformational freedom, i.e., many rotatable bonds, and this explicit inclusion also helps account for basis set superposition error in intermolecular complexes. ${ }^{5}$

For all systems investigated, either Gaussian03 or Gaussian09 was used to perform Density Functional Theory (DFT) calculations with the B3LYP and M06-2X functionals and the 3-21G and Def2-TZVPP basis sets. ${ }^{6-11}$ For the more detailed basis set calculations, the DFT-D3 dispersion correction was also applied. ${ }^{12}$ In each calculation case, the enthalpy at $298.15 \mathrm{~K}$ was calculated with a frequency calculation following the relevant optimization. Repeated optimizations from multiple different starting configurations in select cases showed the expected error for calculated $\Delta H_{\mathrm{A}-\mathrm{B}}^{\circ}$ values to average at or less than $0.1 \mathrm{~kJ} \mathrm{~mol}^{-1}$.

Initial investigations into the preferred interaction conformations were performed with a series of computationally efficient calculations using B3LYP/3-21G. The primary concern was to find if the halogen bonding to the hydroxyl is stronger than that to the conjugated ring electron region of the catalyst. From multiple initial starting configurations, both $\mathrm{C}_{4} \mathrm{~F}_{9} \mathrm{I}$ and $\mathrm{C}_{4} \mathrm{~F}_{9} \mathrm{Br}$ were geometry optimized in the spatial proximity of both the hydroxyl groups and the ring electron density of DTHQ. The favorability for hydroxyl group halogen bonding configurations, $\Delta \Delta H^{\circ}$, was calculated as,

$$
\Delta \Delta H^{\circ}=\Delta H_{\mathrm{A}-\mathrm{B}, \mathrm{hyd}}^{\circ}-\Delta H_{\mathrm{A}-\mathrm{B}, \text { ring }}^{\circ}
$$

Computed $\Delta \Delta H^{\circ}$ values for $\mathrm{C}_{4} \mathrm{~F}_{9} \mathrm{I}$ and $\mathrm{C}_{4} \mathrm{~F}_{9} \mathrm{Br}$ were -3.9 and $-3.8 \mathrm{~kJ} \mathrm{~mol}^{-1}$, respectively. Accepting of electron occupancy to the sigma hole of the substrate halogen atom is favorable from both the 
hydroxyl oxygen and the aromatic ring of DTHQ in the gas phase, as the $\Delta H_{\mathrm{A}-\mathrm{B}}^{\circ}$ values (see Table S2) are negative. In the presence of a non-protolytic explicit solvent, this favorability for halogen bonding with the hydroxyl lone pair electrons will be further amplified due to solute-solvent competition for direct contact with the aromatic ring plane via non-specific dispersion interactions. A quantitative assessment of overall favorability of halogen bonding to the hydroxyl group of DTHQ or other catalysts should take such a competition into account. Such an assessment would require explicit solvent investigation using equilibrium ab initio molecular dynamics or classical explicit solvent molecular dynamics with a force field modified to reproduce halogen bonding interactions, both of which are beyond the scope of this initial investigation.

Table S2. B3LYP/3-21G halogen bond enthalpies of iodo- and bromoperfluoroalkane substrates interacting with DTHQ.

\begin{tabular}{|cc|}
\hline Complex & $\Delta H_{\mathrm{A}-\mathrm{B}}^{\circ} / \mathrm{kJ} \mathrm{mol}^{-1}$ \\
\hline DTHQ $-\mathrm{C}_{4} \mathrm{~F}_{9} \mathrm{I}$ : hydroxl & -7.6 \\
\hline DTHQ $-\mathrm{C}_{4} \mathrm{~F}_{9} \mathrm{I}:$ ring & -3.7 \\
\hline DTHQ $-\mathrm{C}_{4} \mathrm{~F}_{9} \mathrm{Br}$ : hydroxl & -7.3 \\
\hline DTHQ $-\mathrm{C}_{4} \mathrm{~F}_{9} \mathrm{Br}$ : ring & -3.5 \\
\hline
\end{tabular}

To better evaluate the relative halogen bonding interaction strength as a function of both substrate and photocatalyst identity, we scanned over a variety of functionals and basis sets in search of combinations that provided consistent predictions along with element coverage that includes iodine. In the interest of computational efficiency, phenol was used as the halogen bond electron donor while the set of all possible halogen diatomic molecules were used as electron acceptors. Results for $\Delta H_{\mathrm{A}-\mathrm{B}}^{\circ}$ values using B3LYP/Def2-TZVPP and M06-2X/Def2-TZVPP are shown in Table S3.

Expectedly, $\Delta H_{\mathrm{A}-\mathrm{B}}^{\circ}$ grows stronger with increasing quantum number, this given the increasingly deep $\sigma$ hole. ${ }^{13-15}$ Within specific halogen bond series, changing the identity from more to less electronegative covalently bonded atoms on the diatomic molecule leads to a corresponding decrease in $\Delta H_{\mathrm{A}-\mathrm{B}}^{\circ}$. This is again expected given the halogen bond accepting atom is able to gain electron density from the more electron rich covalently bonded atom, leading to a subsequent decreased drive for charge transfer from the halogen bond donor catalyst. These trends are reproduced by both B3LYP and M06-2X. Both functionals struggle to maintain a systematic trend when fluorine is acting as the halogen bond acceptor, and this is not unexpected given its generally weak partnering in this regard. ${ }^{13}$ In the B3LYP calculations, the interactions involving fluorine interacting with the hydroxyl group are considerably weaker, sometimes positive in the case of $\mathrm{FCl}$, consistent with the expectation that fluorine does not participate in or form halogen bonds. The $\Delta H_{\mathrm{A}-\mathrm{B}}^{\circ}$ in these cases are likely less due to a directional halogen bond and more due to nonspecific dispersion interactions between the molecular pair. $\Delta H_{\mathrm{A}-\mathrm{B}}^{\circ}$ values with the M06-2X functional are more exaggerated than B3LYP, particularly in the jump to iodine accepting the halogen bond. M06-2X appears more sensitive in changes to the halogen atom directly involved 
in the halogen bond, but it is less sensitive to electron donation/withdrawing from covalently bonded sources, except in the case that the source is a fluorine atom.

Table S3. Halogen bond enthalpies for halogen diatomic molecules with the hydroxyl group of phenol, these with the Def2-TZVPP basis set and DFT-D3 long-range interaction.

\begin{tabular}{|ccc|}
\hline \multirow{2}{*}{ Complex } & B3LYP & M06-2X \\
\cline { 2 - 3 } & $\Delta H_{\mathrm{A}-\mathrm{B}}^{\circ} / \mathrm{kJ} \mathrm{mol}^{-1}$ & $\Delta H_{\mathrm{A}-\mathrm{B}}^{\circ} / \mathrm{kJ} \mathrm{mol}^{-1}$ \\
\hline Phenol - $\mathrm{F}_{2}$ & -5.8 & -8.4 \\
\hline Phenol - F-Cl & 0.4 & -10.2 \\
\hline Phenol - F-Br & -2.1 & -7.6 \\
\hline Phenol - F-I & -2.4 & -6.0 \\
\hline Phenol - Cl-F & -20.6 & -27.1 \\
\hline Phenol - Cl 2 & -11.0 & -16.6 \\
\hline Phenol - Cl-Br & -9.0 & -14.9 \\
\hline Phenol - Cl-I & -5.7 & -11.2 \\
\hline Phenol - Br-F & -28.6 & -31.7 \\
\hline Phenol - Br-Cl & -17.7 & -24.1 \\
\hline Phenol - Br 2 & -15.2 & -22.0 \\
\hline Phenol - Br-I & -11.3 & -17.5 \\
\hline Phenol - I-F & -34.1 & -48.0 \\
\hline Phenol - I-Cl & -24.6 & -35.0 \\
\hline Phenol - - - $\mathrm{Br}$ & -22.8 & -32.1 \\
\hline Phenol - $\mathrm{I}_{2}$ & -17.2 & -26.7 \\
\hline
\end{tabular}

With this baseline halogen bonding series established, we swapped phenol for DTHQ and computed halogen bond enthalpies for the single halogen diatomic molecules. This provides an indication of how functionalization of the central ring and changes to the local environment around the hydroxyl group alter the halogen bond strength. As a final step, the halogen bond enthalpy was calculated for DTHQ complexes with $\mathrm{C}_{4} \mathrm{~F}_{9} \mathrm{Cl}, \mathrm{C}_{4} \mathrm{~F}_{9} \mathrm{Br}$, and $\mathrm{C}_{4} \mathrm{~F}_{9} \mathrm{I}$ to see where the perfluoroalkane substrates fall in this series and provide comparable data points for the synthesis efforts. $\Delta H_{\mathrm{A}-\mathrm{B}}^{\circ}$ values for these systems are collected and displayed in Table S4.

Table S4. Halogen bond enthalpies for halogen diatomic and fluoroalkane molecules with the hydroxyl group of DTHQ, these with the Def2-TZVPP basis set and DFT-D3 long-range interaction correction.

\begin{tabular}{|ccc|}
\hline \multirow{2}{*}{ Complex } & B3LYP & M06-2X \\
\cline { 2 - 3 } & $\Delta H_{\mathrm{A}-\mathrm{B}}^{\circ} / \mathrm{kJ} \mathrm{mol}^{-1}$ & $\Delta H_{\mathrm{A}-\mathrm{B}}^{\circ} / \mathrm{kJ} \mathrm{mol}^{-1}$ \\
\hline $\mathrm{DTHQ}-\mathrm{F}_{2}$ & -4.1 & -12.7 \\
\hline $\mathrm{DTHQ}-\mathrm{Cl}_{2}$ & -7.9 & -24.5 \\
\hline $\mathrm{DTHQ}-\mathrm{Br}_{2}$ & -11.8 & -25.7 \\
\hline $\mathrm{DTHQ}-\mathrm{I}_{2}$ & -14.5 & -31.0 \\
\hline DTHQ $-\mathbf{C}_{4} \mathbf{F} \mathbf{F} \mathbf{C l}$ & $\mathbf{- 6 . 5}$ & -23.0 \\
\hline DTHQ $-\mathbf{C}_{4} \mathbf{F} \mathbf{F} \mathbf{B r}$ & $\mathbf{- 7 . 6}$ & -25.7 \\
\hline DTHQ $-\mathbf{C}_{\mathbf{4}} \mathbf{F} \mathbf{F} \mathbf{I}$ & $\mathbf{- 1 1 . 4}$ & -27.7 \\
\hline
\end{tabular}


In changing from phenol to DTHQ, there is steric occlusion about the hydroxyl lone pair electrons due to the presence of the adjacent tertbutyl functional group. This is clearly observed in the B3LYP calculations, but not for the M06-2X functional, which actually grow to be a little greater than $5 \mathrm{~kJ} \mathrm{~mol}^{-1}$. This is an unclear result and inconsistent with physical expectations, one that is also not noticeably consistent with the visualized optimized geometries shown in Figure S6. Both the B3LYP and M06-2X optimizations result in similar geometries, with the $\mathrm{I}_{2}$ molecule deflected out of optimal alignment with the hydroxyl oxygen with the presence of the tertbutyl group in DTHQ. M06-2X has a shorter halogen bond length than B3LYP but it is difficult to see this contributing to the significant increase in strength of halogen bonding for M06-2X in moving from phenol to DTHQ. A general preference for attractive non-covalent dispersion interactions in the more heavily statistically fit M06-2X functional or imbalance with the applied DFT-D3 dispersion correction (not present in the original developmental fit of the M06-2X functional) are the most likely sources for this result, and an expanded investigation would be needed to discern among these candidate options. Given this physical discrepancy, only the B3LYP calculation results are used in the interpretations in the current study.

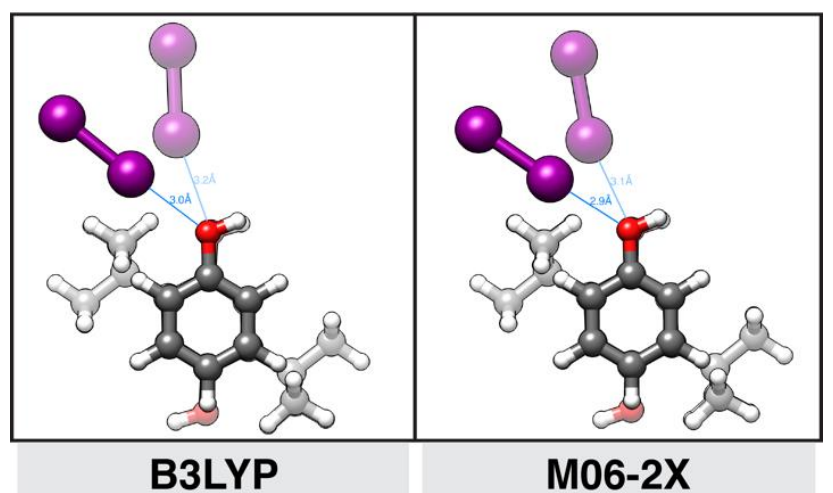

Figure S7. A visual comparison of halogen bonding geometries for B3LYP/Def2-TZVPP/D3 and M06-2X/Def2-TZVPP/D3 of the phenol-I $\mathrm{I}_{2}$ (lighter color) and DTHQ- $\mathrm{I}_{2}$ (darker color) complexes. The steric role of the tertbutyl group is apparent in hindering the potential halogen bonding interaction, and increased activation through halogen bonding could be achieved with a less crowded local space.

The B3LYP/Def2-TZVPP/D3 results in Table S4 make a couple of things clear. First, the halogen bond is about $4 \mathrm{~kJ} \mathrm{~mol}^{-1}$ weaker when formed between DTHQ and a bromofluoroalkane than an iodofluoroalkane. This likely contributes to a lessened activation in the synthetic pathway and a corresponding decrease in the overall yield of functionalization. Second, the fluoroalkane chain is less electron withdrawing than a covalently bonded like-halogen, resulting in a decreased overall interaction strength. The nature of the fluoroalkyl chain will likely have an influence on the halogen bond driven activation process, though it is likely to be more subtle than changes in halogen or catalyst identity. Finally, there is a universal weakening of the halogen bond strength of roughly $3 \mathrm{~kJ} \mathrm{~mol}^{-1}$ (for simple diatomic halogen molecules) due to steric occlusion of the 
hydroxyl group in DTHQ over phenol. This indicates that there is potential to tune the efficacy of the described reaction pathway by structural modifications of the catalyst. 
52

XYZ File B3LYP/Def2-TZVPP/D3 optimized DTHQ-C4F9Cl complex

C

6.72400

$-1.35500$

0.21800

6.54800

$-2.85800$

$-0.03500$

7.34400

$-1.19500$

1. 62000

7.69900

$-0.81900$

$-0.84700$

$-0.59800$

0.14200

4.19300

$-1.24900$

$-0.13100$

2.96500

$-0.60200$

$-0.20900$

2.86500

0.78100

$-0.01100$

1. 43200

0.26200

5.29700

0.78500

1. 54100

0.34000

1.00600

$-0.08700$

0.56300

3.04200

0.97900

1.71400

1.38800

0.17100

0.92400

1. 51200

$-1.33700$

1.82900

6.14200

$-3.05300$

$-3.34300$

$-3.31600$

$-1.49200$

0.61500

$-0.48600$

$-1.03000$

0.03100

5.89100

$-0.15100$

0.70700

7.53900

$-1.60500$

1. 85300

2.37900

1.66400

8.28800

$-1.74500$

0.24000

$-0.70500$

8.64300

$-1.36700$

$-0.78500$

7.28400

$-0.96400$

$-1.84700$

0.72300

0.42500

0.84100

1. 97800

0.98000

2. 50100

$-0.05200$

0.91800

1. 55300

$-0.56900$

2.37200

3.50300

0.10400

1.16700

2.11900

3. 23400

1. 81700

$-2.24200$

0.74600

0.34600

$-1.74100$

$-0.02800$

1.92100

$-1.53300$

$-2.26200$

$-0.57100$

$-2.31900$

$-0.29500$

$-1.65300$

$-0.99700$

$-1.21300$

$-0.88400$

0.17500

$-0.17200$

0.72400

1. 99200

2. 44000

$-0.14600$

0.75100

0.58600

1.69900

2.03900

2.95700

0.40000

1.05400

$-1.40000$

0.31700

1.09500

0.04700

$-0.81400$

1.07800

$-2.11100$

$-1.14300$

$-0.18700$ 
52

XYZ File B3LYP/Def2-TZVPP/D3 optimized DTHQ-C4F9Br complex

C

6.85700

$-1.58100$

0.27800

6.43600

$-3.05200$

0.16900

7.53600

$-1.39700$

1. 64900

7.87200

$-1.30800$

$-0.84900$

$-0.62800$

0.15300

4.36500

$-1.09700$

3. 25500

$-0.26800$

$-0.03600$

3.38500

1.12400

$-0.15200$

4.68400

1.59400

$-0.08400$

0.76500

0.10500

2.07700

0.22100

2.19300

1.81000

$-0.20600$

3.55000

0.93300

2.61300

1.89400

$-0.10300$

1.51300

7.04300

1.31900

2.00700

$-0.83100$

$-1.57600$

0.40500

5.97500

$-3.26800$

$-0.33600$

7.32200

$-3.68000$

$-0.79700$

5.73600

$-3.32900$

0.26800

0.96100

7.89800

$-0.38000$

1.78200

2.45300

1. 72800

8.38400

8.25000

$-2.08200$

$-0.80800$

8.71500

$-0.28900$

$-0.75100$

7.40800

$-1.99700$

$-1.82400$

2. 27600

0.42600

4.85500

2.66000

0.16600

0.84600

0.78000

0.93700

2.02300

1.89800

0.32200

2. 46400

0.81600

3.30900

3.82400

$-0.89800$

4.17600

3.77000

2.16100

$-0.20100$

0.86100

0.87000

$-2.37600$

2.55100

$-1.73300$

1.18500

$-1.78800$

$-1.64200$

2. 09500

$-2.16400$

$-0.34300$

$-0.09800$

$-0.76000$

$-0.69400$

$-0.96900$

$-0.80100$

0.11400

0.07200

$-3.81000$

0.11800

1. 01000

1.05900

$-0.07500$

0.97700

0.65700

0.49800

2.20200

2. 25100

0.97500

0.58200

$-1.29800$

0.05700

1.36300

$-0.10900$

$-0.28700$

1.33100

$-0.84500$

$-2.07500$

$-2.19000$

$-0.36800$ 
52

XYZ File B3LYP/Def2-TZVPP/D3 optimized DTHQ-C4F9I complex

C

6.96200

$-1.70800$

6.37800

$-3.12700$

0.23500

7.75200

$-1.54700$

0.22400

7.91900

$-1.59500$

1. 54800

5.87300

$-0.63600$

4.52100

$-0.96600$

3.50300

$-0.02400$

.96700

3.78500

1.34700

1.67700

0.73600

2. 41700

2.31000

3. 83800

2. 25200

1. 15500

$-0.45300$

$-3.32600$

$-3.84500$

$-3.29500$

0.14300

0.06200

$-0.02600$

$-0.04000$

0.04200

0.13100

$-0.14100$

1.06000

$-0.13000$

$-1.46200$

0.21000

$-0.10300$

$-0.69700$

0.28800

1.07400

1.60900

2.40700

1. 60500

$-1.66400$

$-1.00000$

8.40800

$-0.62500$

$-0.89300$

7.37200

$-2.37000$

$-1.90100$

2.11600

0.19200

2. 72000

1. 32700

0.03800

1.27600

2. 49900

1.12700

3.05700

1.99000

0.94400

4.00600

0.96200

3.94200

4.55300

4.04000

2.41000

$-0.97700$

3.82100

2.58700

1. 26200

$-0.20000$

0.79300

1.48100

2. 99300

$-2.31100$

$-1.55500$

1.11800

$-1.41400$

$-1.51200$

4. 23200

$-2.00800$

$-0.08100$

0.06600

$-1.02300$

$-0.27500$

$-0.35700$

$-0.96100$

$-0.52800$

$-3.08900$

0.06000

0.10000

$-5.60100$

$-0.31400$

$-0.08600$

0.60300

0.69800

0.25100

0.36800

0.45700

2.01400

1.88600

0.37800

$-0.22500$

$-1.39300$

$-1.57800$

0.33100

1.42000

1.26600

$-0.47000$

$-1.14100$

$-1.82300$

$-3.40000$

$-2.14400$

0.10000 


\section{References}

1. Zhu, E.; Liu, X.-X.; Wang, A.-J.; Mao, T.; Zhao, L.; Zhang, X.; He, C.-Y. Visible light promoted fluoroalkylation of alkenes and alkynes using 2-bromophenol as a catalyst. Chem. Commun. 2019, 55, 12259-12262.

2. Liang, K.; Liu, Q.; Shen, L.; Li, X.; Wei, D.; Zheng, L.; Xia, C. Intermolecular oxyarylation of olefins with aryl halides and TEMPOH catalyzed by the phenolate anion under visible light. Chem. Sci. 2020, 11, 6996-7002.

3. Liang, K.; Li, T.; Li, N.; Zhang, Y.; Shen, L.; Ma, Z.; Xia, C. Redox-neutral photochemical Heck-type arylation of vinylphenols activated by visible light. Chem. Sci. 2020, 11, 21302135.

4. Benesi, H. A.; Hildebrand, J. H. A Spectrophotometric Investigation of the Interaction of Iodine with Aromatic Hydrocarbons. J. Am. Chem. Soc. 1949, 71, 2703-2707.

5. Šponer, J.; Jurečka, P.; Hobza, P., Accurate Interaction Energies of Hydrogen-Bonded Nucleic Acid Base Pairs. J. Am. Chem. Soc. 2004, 126, 10142-10151.

6. M. J. Frisch, G. W. T., H. B. Schlegel, G. E. Scuseria, M. A. Robb, J. R. Cheeseman, J. A. Montgomery, Jr., T. Vreven, K. N. Kudin, J. C. Burant, J. M. Millam, S. S. Iyengar, J. Tomasi, V. Barone, B. Mennucci, M. Cossi, G. Scalmani, N. Rega, G. A. Petersson, H. Nakatsuji, M. Hada, M. Ehara, K. Toyota, R. Fukuda, J. Hasegawa, M. Ishida, T. Nakajima, Y. Honda, O. Kitao, H. Nakai, M. Klene, X. Li, J. E. Knox, H. P. Hratchian, J. B. Cross, V. Bakken, C. Adamo, J. Jaramillo, R. Gomperts, R. E. Stratmann, O. Yazyev, A. J. Austin, R. Cammi, C. Pomelli, J. W. Ochterski, P. Y. Ayala, K. Morokuma, G. A. Voth, P. Salvador, J. J. Dannenberg, V. G. Zakrzewski, S. Dapprich, A. D. Daniels, M. C. Strain, O. Farkas, D. K. Malick, A. D. Rabuck, K. Raghavachari, J. B. Foresman, J. V. Ortiz, Q. Cui, A. G. Baboul, S. Clifford, J. Cioslowski, B. B. Stefanov, G. Liu, A. Liashenko, P. Piskorz, I. Komaromi, R. L. Martin, D. J. Fox, T. Keith, M. A. Al-Laham, C. Y. Peng, A. Nanayakkara, M. Challacombe, P. M. W. Gill, B. Johnson, W. Chen, M. W. Wong, C. Gonzalez, and J. A. Pople Gaussian 03, Revision D.01; Gaussian, Inc.: Wallingford CT, 2004.

7. Becke, A. D., Density-Functional Thermochemistry. III. The Role of Exact Exchange. $J$. Chem. Phys. 1993, 98, 5648-5652.

8. Lee, C.; Yang, W.; Parr, R. G., Development of the Colle-Salvetti Correlation-Energy Formula into a Functional of the Electron Density. Phys. Rev. B 1988, 37, 785-789. 
9. Stephens, P. J.; Devlin, F. J.; Chabalowski, C. F.; Frisch, M. J., Ab Initio Calculation of Vibrational Absorption and Circular Dichroism Spectra Using Density Functional Force Fields. J. Phys. Chem. 1994, 98, 11623-11627.

10. Zhao, Y.; Truhlar, D. G., The M06 Suite of Density Functionals for Main Group Thermochemistry, Thermochemical Kinetics, Noncovalent Interactions, Excited States, and Transition Elements: Two New Functionals and Systematic Testing of Four M06-Class Functionals and 12 Other Functionals. Theor. Chem. Acc. 2008, 120, 215-241.

11. Weigend, F.; Ahlrichs, R., Balanced Basis Sets of Split Valence, Triple Zeta Valence and Quadruple Zeta Valence Quality for H to Rn: Design and Assessment of Accuracy. Phys. Chem. Chem. Phys. 2005, 7, 3297-3305.

12. Grimme, S.; Antony, J.; Ehrlich, S.; Krieg, H., A Consistent and Accurate Ab Initio Parametrization of Density Functional Dispersion Correction (DFT-D) for the 94 Elements HPu. J. Chem. Phys. 2010, 132, 154104.

13. Cavallo, G.; Metrangolo, P.; Milani, R.; Pilati, T.; Priimagi, A.; Resnati, G.; Terraneo, G., The Halogen Bond. Chem. Rev. 2016, 116, 2478-2601.

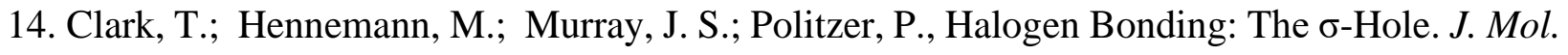
Model. 2007, 13, 291-296.

15. Bundhun, A.; Ramasami, P.; Murray, J. S.; Politzer, P., Trends in $\sigma$-Hole Strengths and Interactions of F3MX Molecules $(\mathrm{M}=\mathrm{C}, \mathrm{Si}, \mathrm{Ge}$ and $\mathrm{X}=\mathrm{F}, \mathrm{Cl}, \mathrm{Br}, \mathrm{I})$. J. Mol. Model. 2013, 19, 2739-2746. 


\section{N. NMR Spectra}

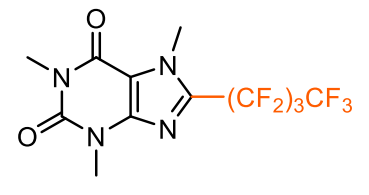

1,3,7-Trimethyl-8-(perfluorobutyl)-3,7-dihydro-1H-purine-2,6-

dione (1)

${ }^{1}$ H NMR: (400 MHz, $\left.\mathrm{CDCl}_{3}\right)$

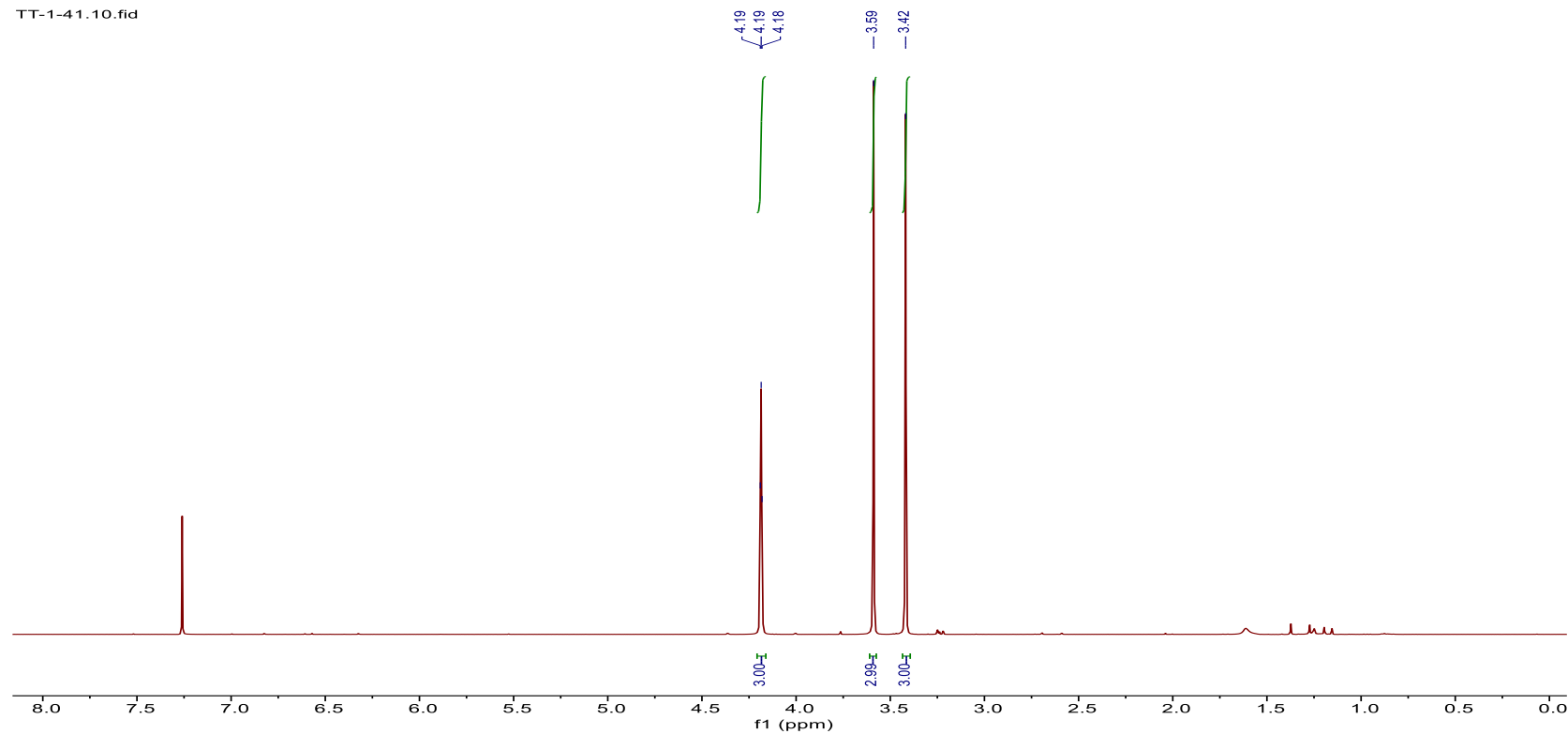

${ }^{13}$ C NMR: (201 MHz, $\mathrm{CDCl}_{3}$ )

TT-1-41C.1.fid
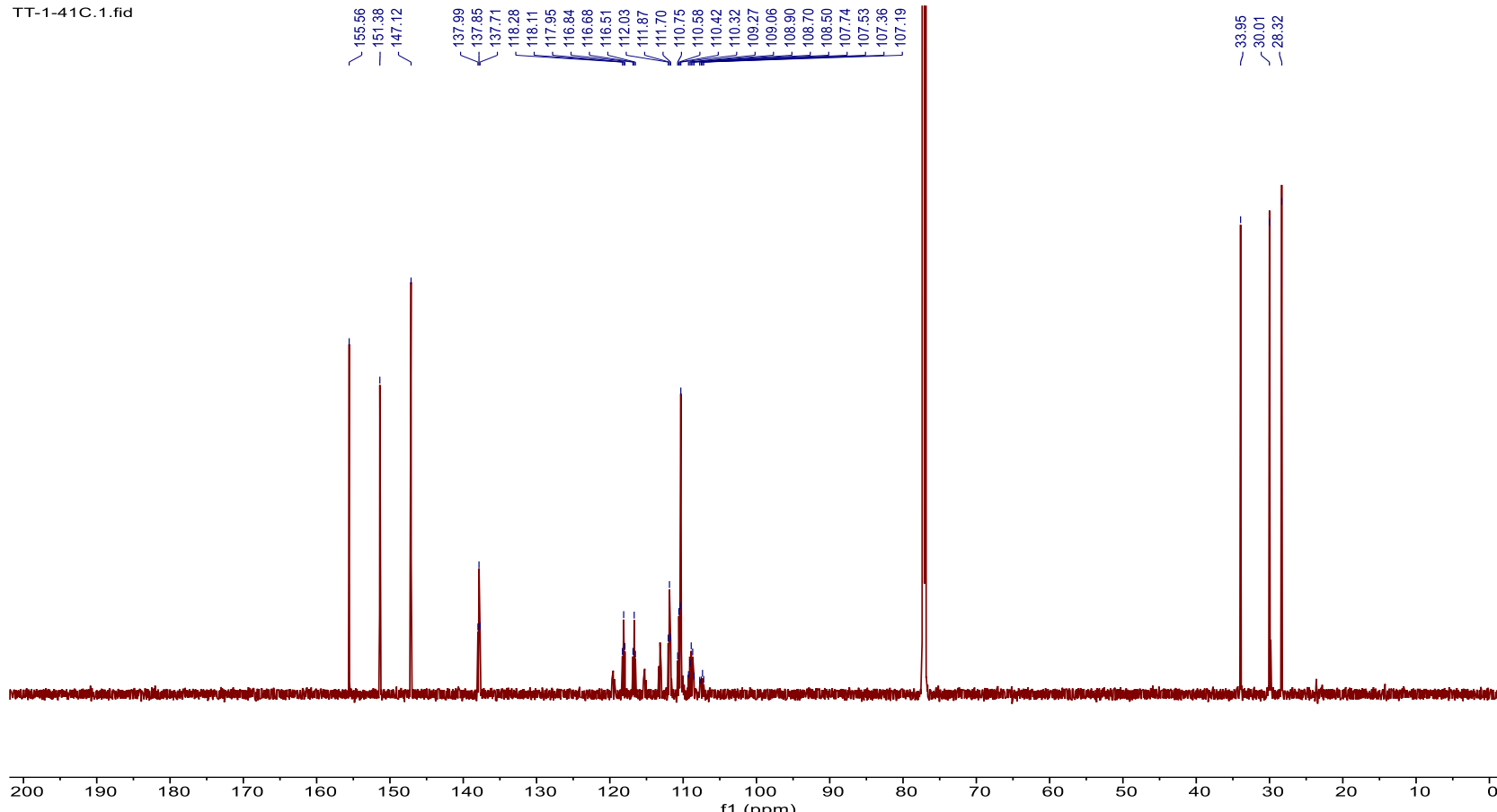

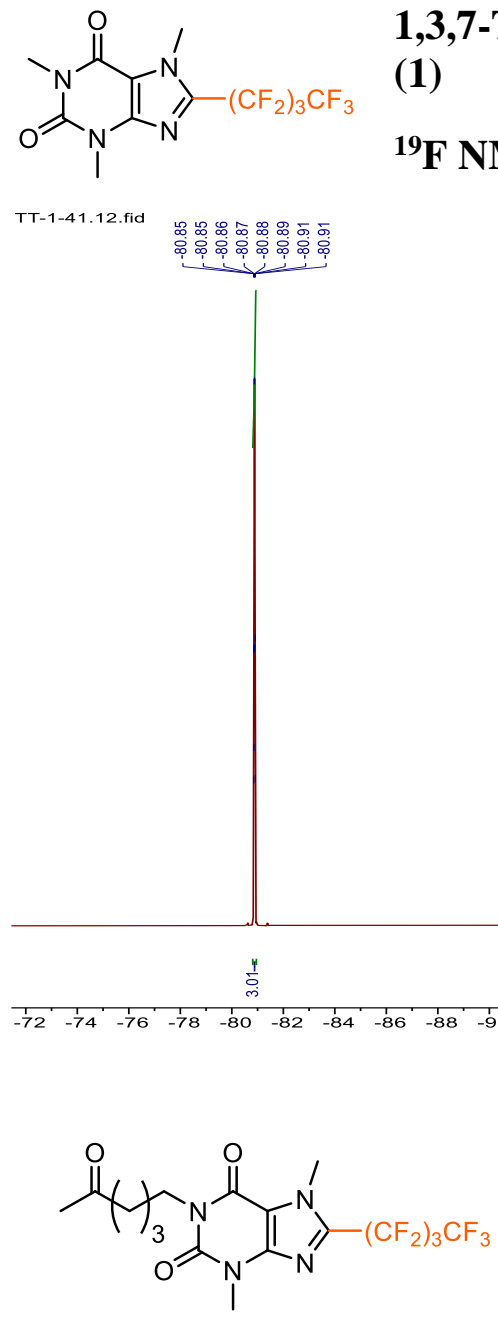

1,3,7-Trimethyl-8-(perfluorobutyl)-3,7-dihydro-1H-purine-2,6-dione (1)

${ }^{19}$ F NMR: $\left(376 \mathrm{MHz}, \mathrm{CDCl}_{3}\right)$

TT-1-88-1H.10.fid

3,7-Dimethyl-1-(5-oxohexyl)-8-(perfluorobutyl)-3,7-dihydro$1 H$-purine-2,6-dione (2)

${ }^{1}$ H NMR: $\left(400 \mathrm{MHz}, \mathrm{CDCl}_{3}\right)$

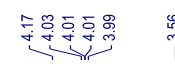

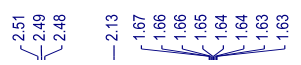

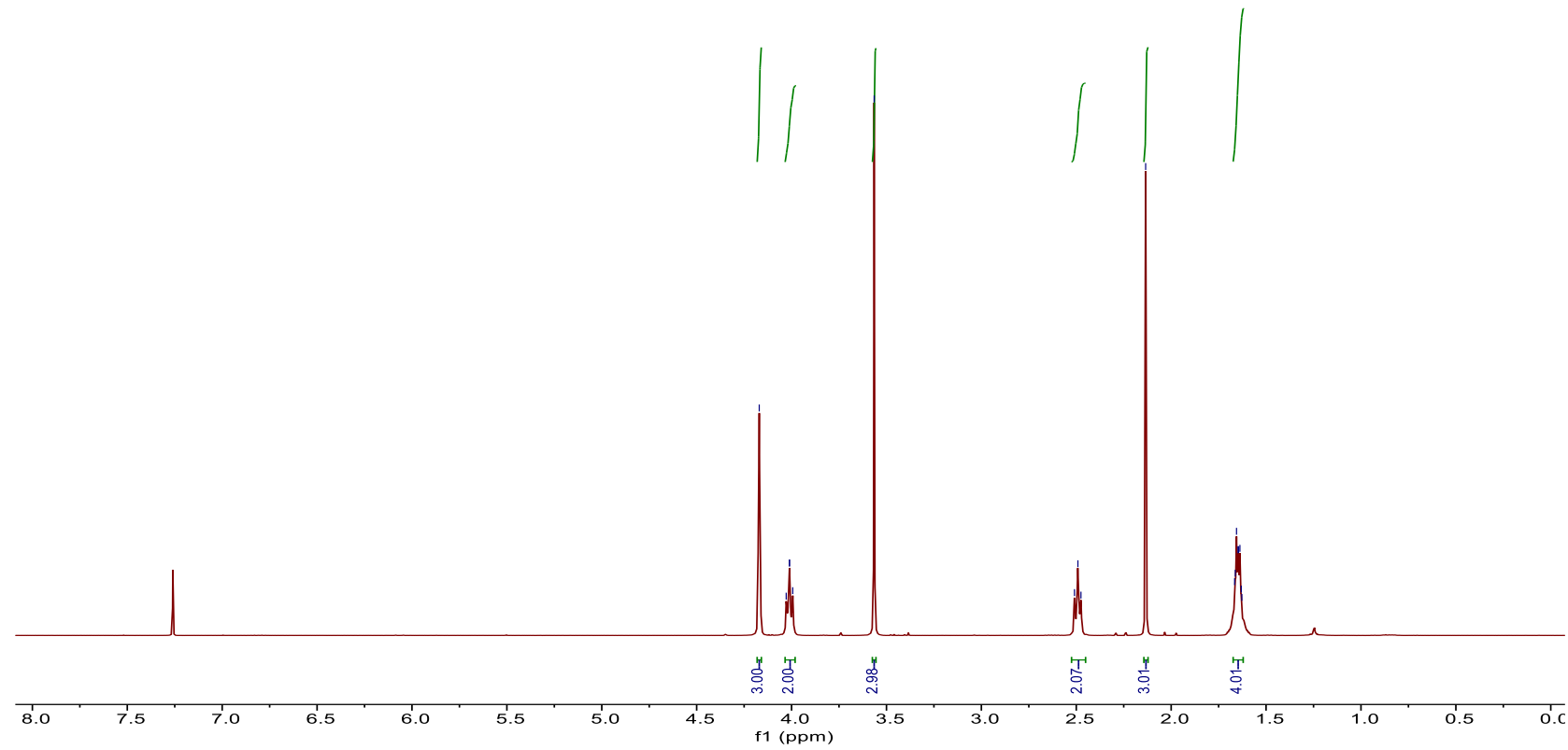




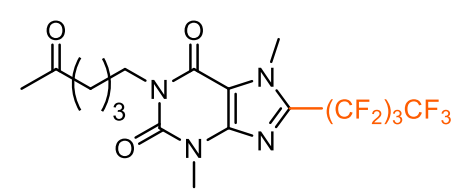

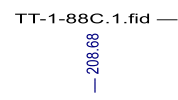

3,7-Dimethyl-1-(5-oxohexyl)-8-(perfluorobutyl)-3,7-dihydro-1Hpurine-2,6-dione (2)

${ }^{13}$ C NMR: (201 MHz, $\left.\mathrm{CDCl}_{3}\right)$

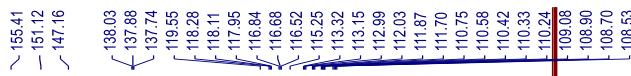

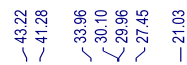

idiente
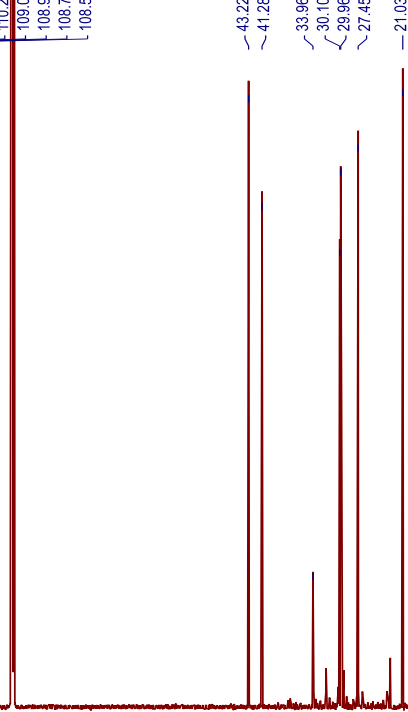

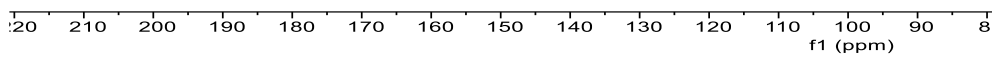
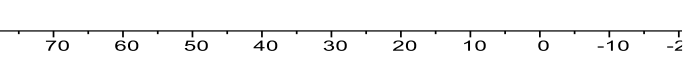

${ }^{19}$ F NMR: (376 MHz, $\mathrm{CDCl}_{3}$ )

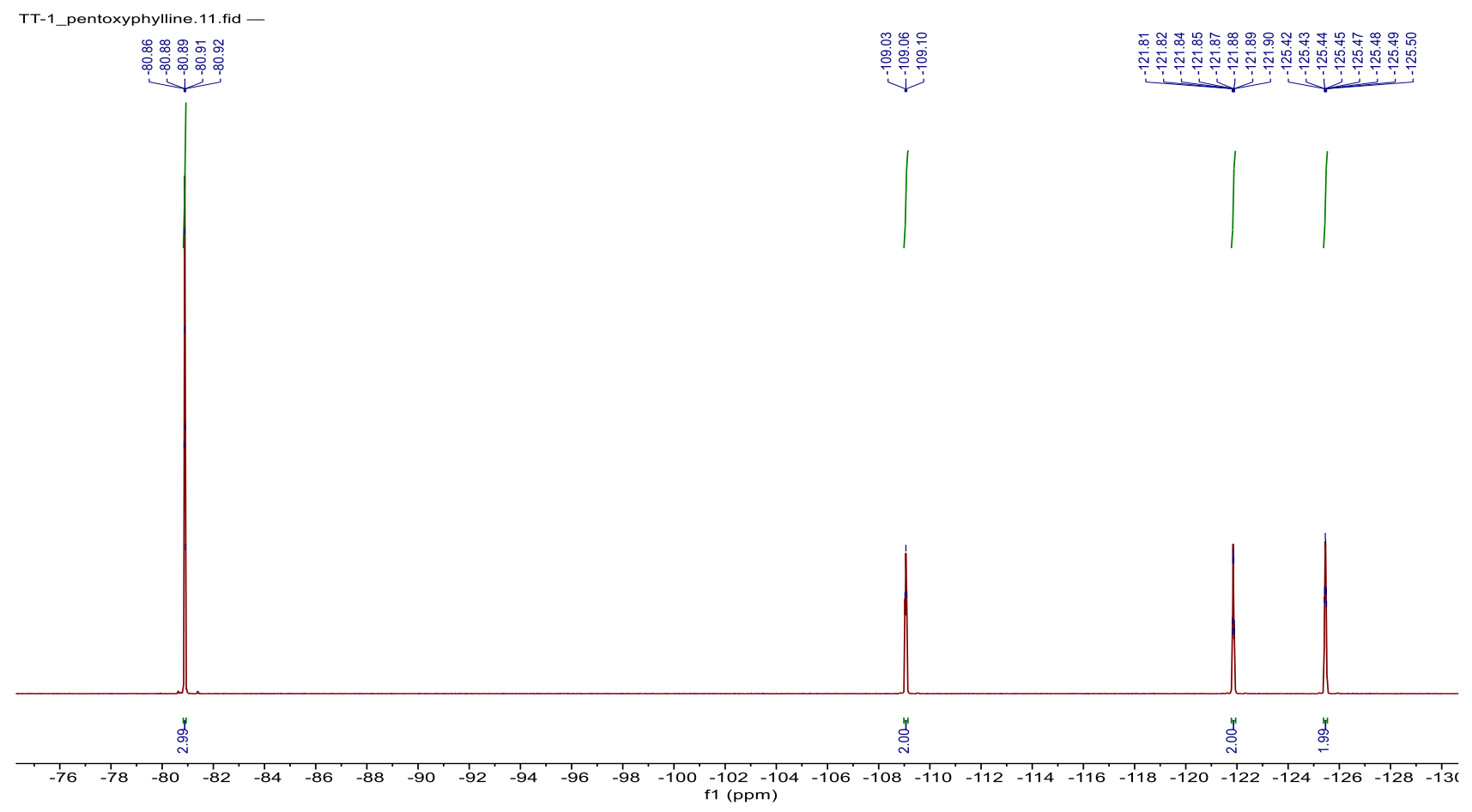


$\prod_{\mathrm{O}}^{\mathrm{H}}$

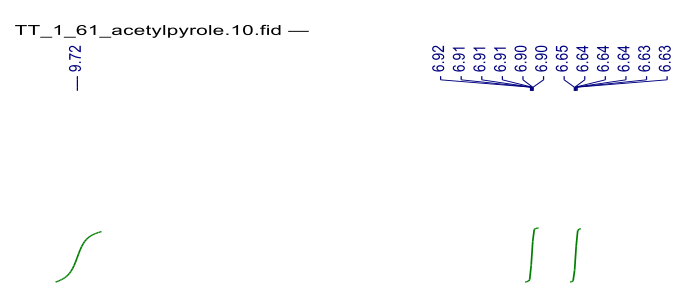

1-(5-(Perfluorobutyl)-1H-pyrrol-2-yl)ethan-1-one (3)

${ }^{1}$ H NMR: (400 MHz, $\left.\mathrm{CDCl}_{3}\right)$

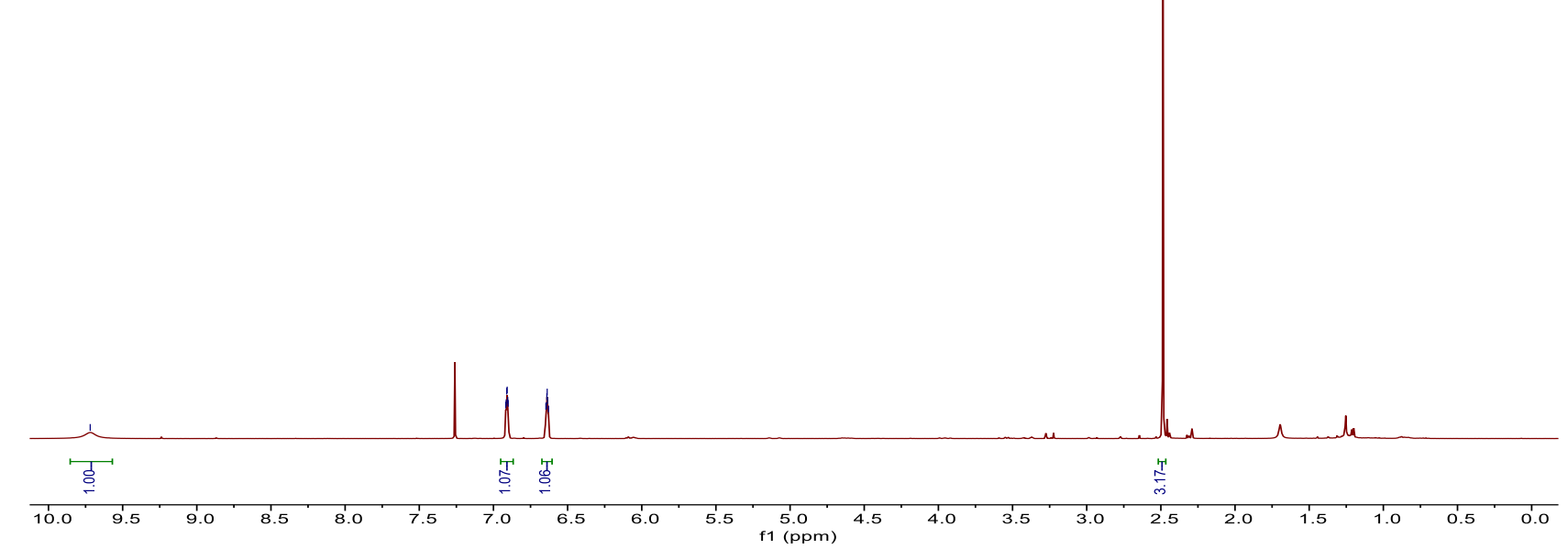

${ }^{13}$ C NMR: (201 MHz, $\left.\mathrm{CDCl}_{3}\right)$

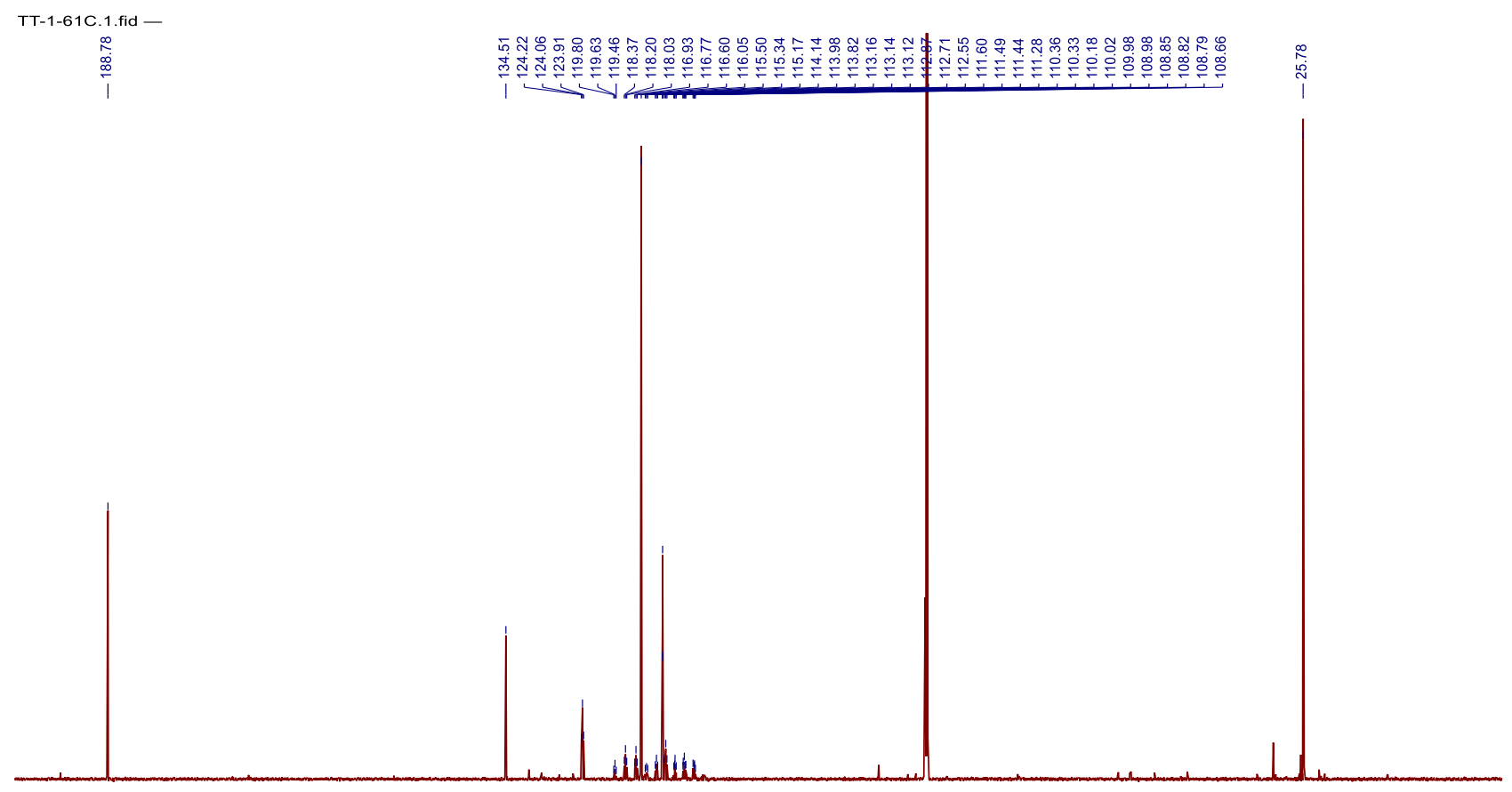

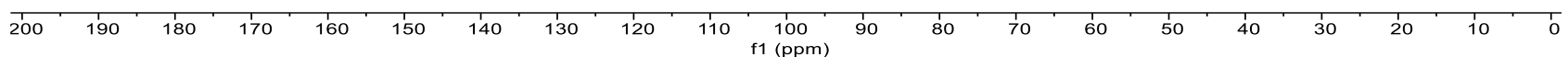


$\prod_{\mathrm{O}} \prod_{\left(\mathrm{CF}_{2}\right)_{3} \mathrm{CF}_{3}}$

1-(5-(Perfluorobutyl)-1H-pyrrol-2-yl)ethan-1-one (3)

${ }^{19}$ F NMR: $\left(376 \mathrm{MHz}, \mathrm{CDCl}_{3}\right)$

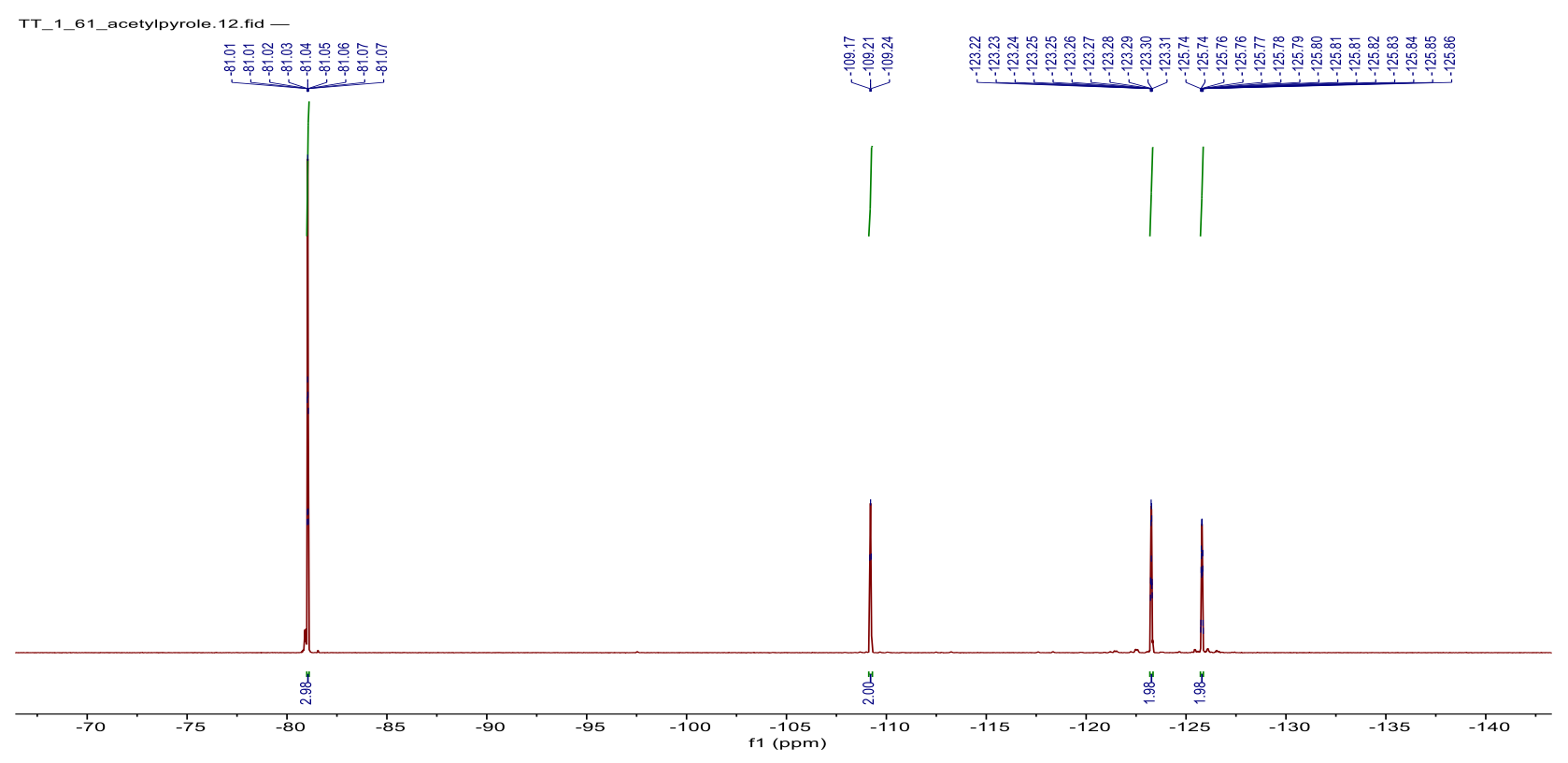

$\begin{array}{ll}\text { 3-Methyl-2-(perfluorobutyl)benzo[b]thiophene (4) } & \\ { }_{\mathrm{S}} & \end{array}$

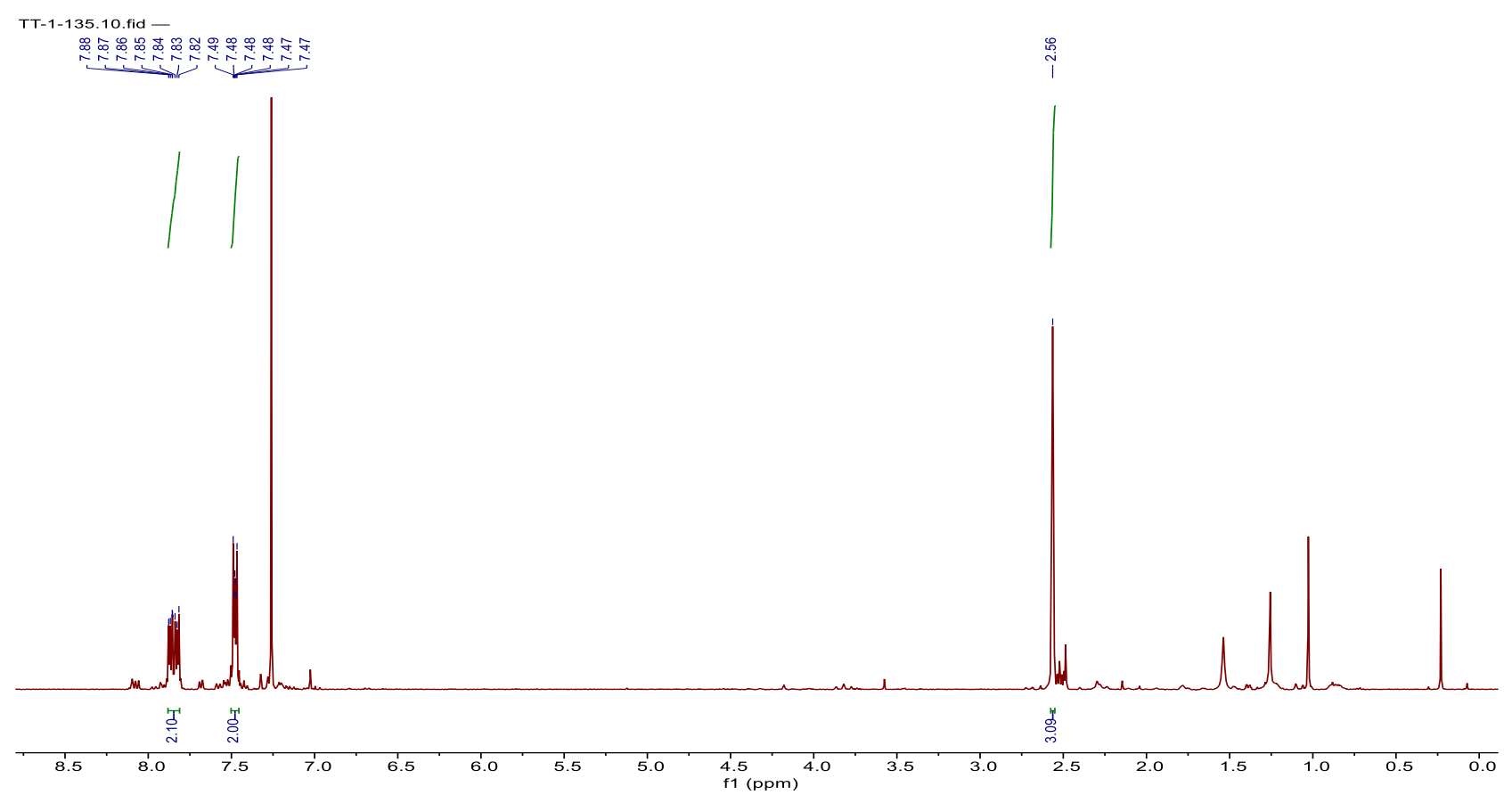




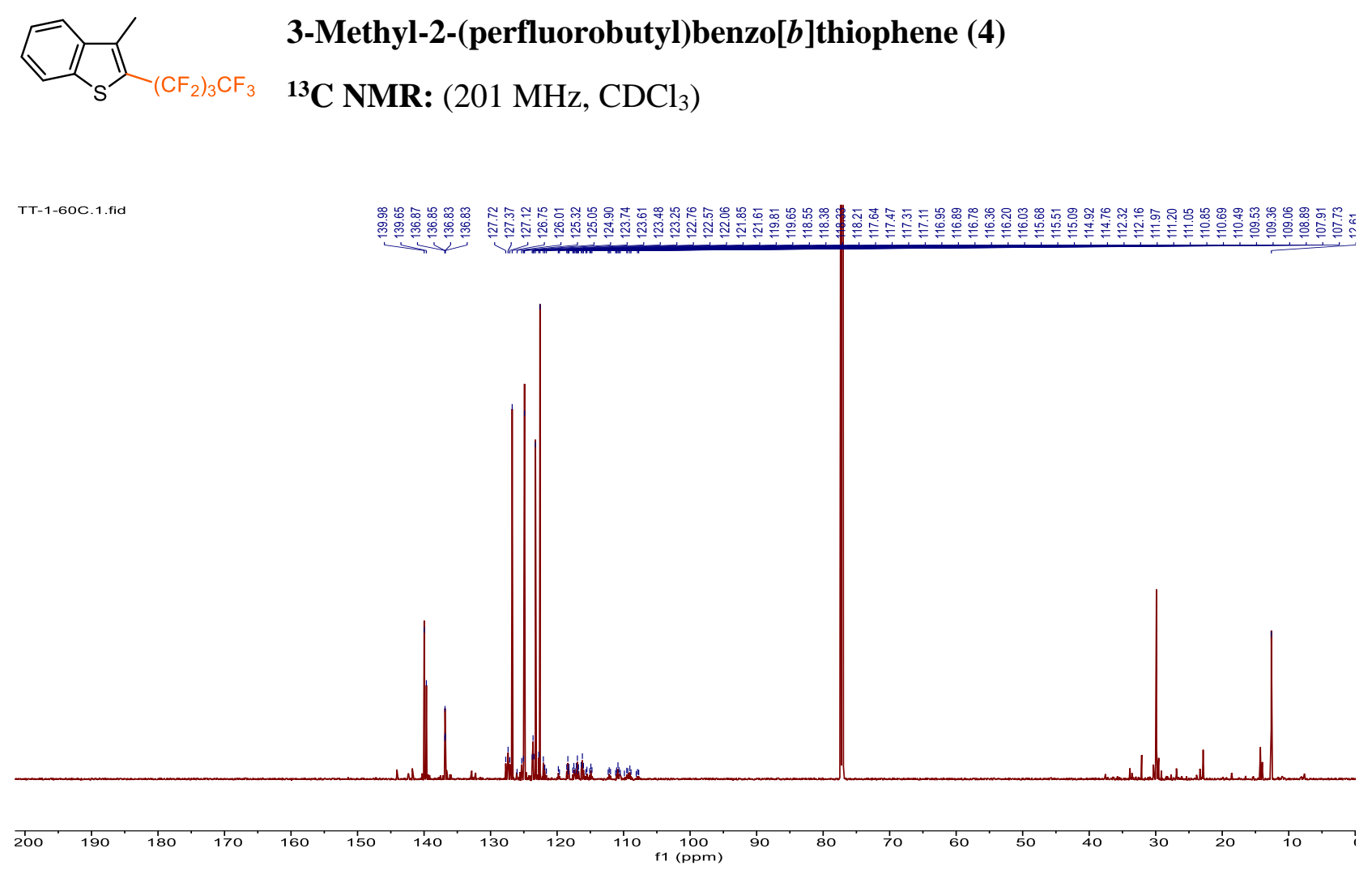

${ }^{19}$ F NMR: (376 MHz, $\mathrm{CDCl}_{3}$ )

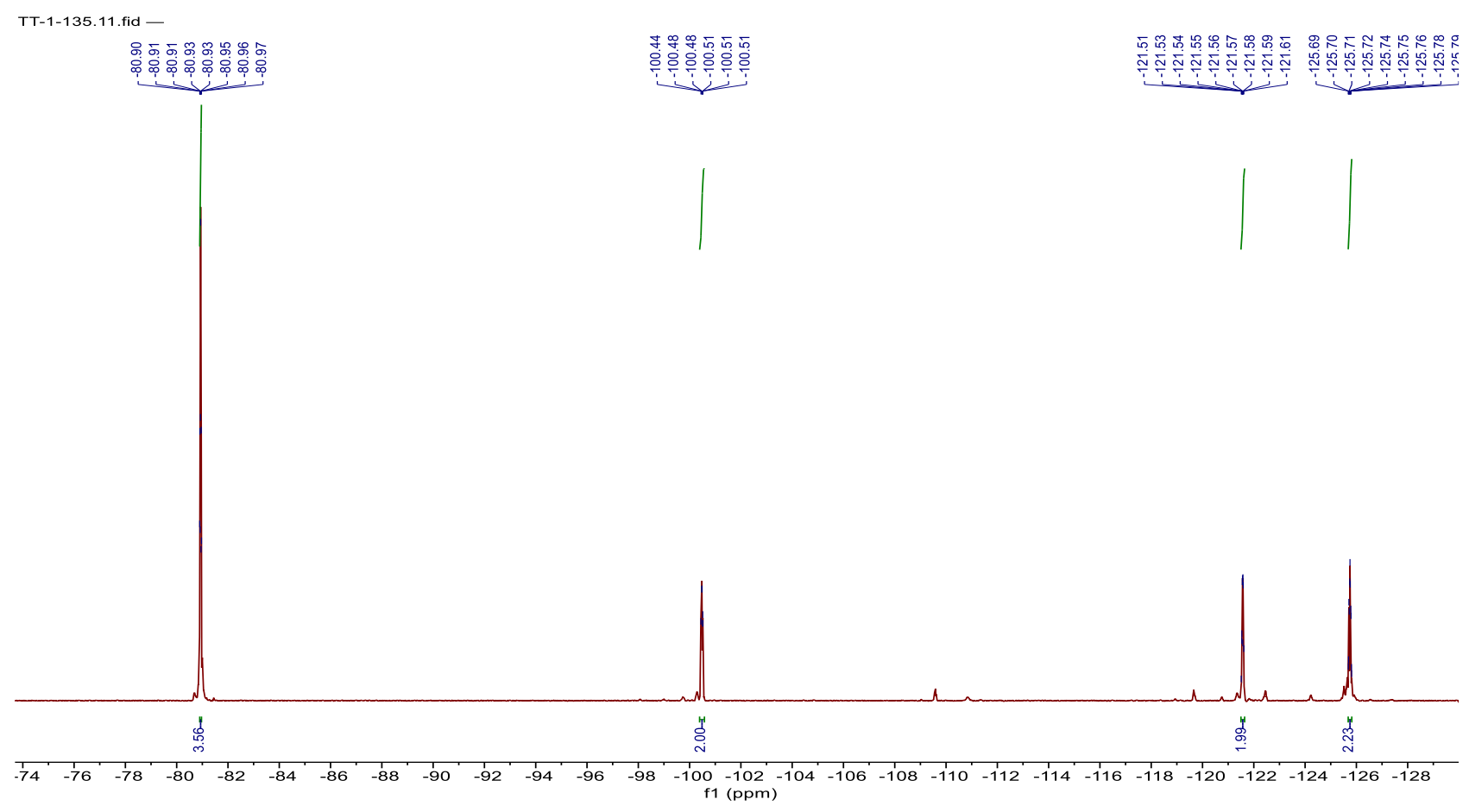




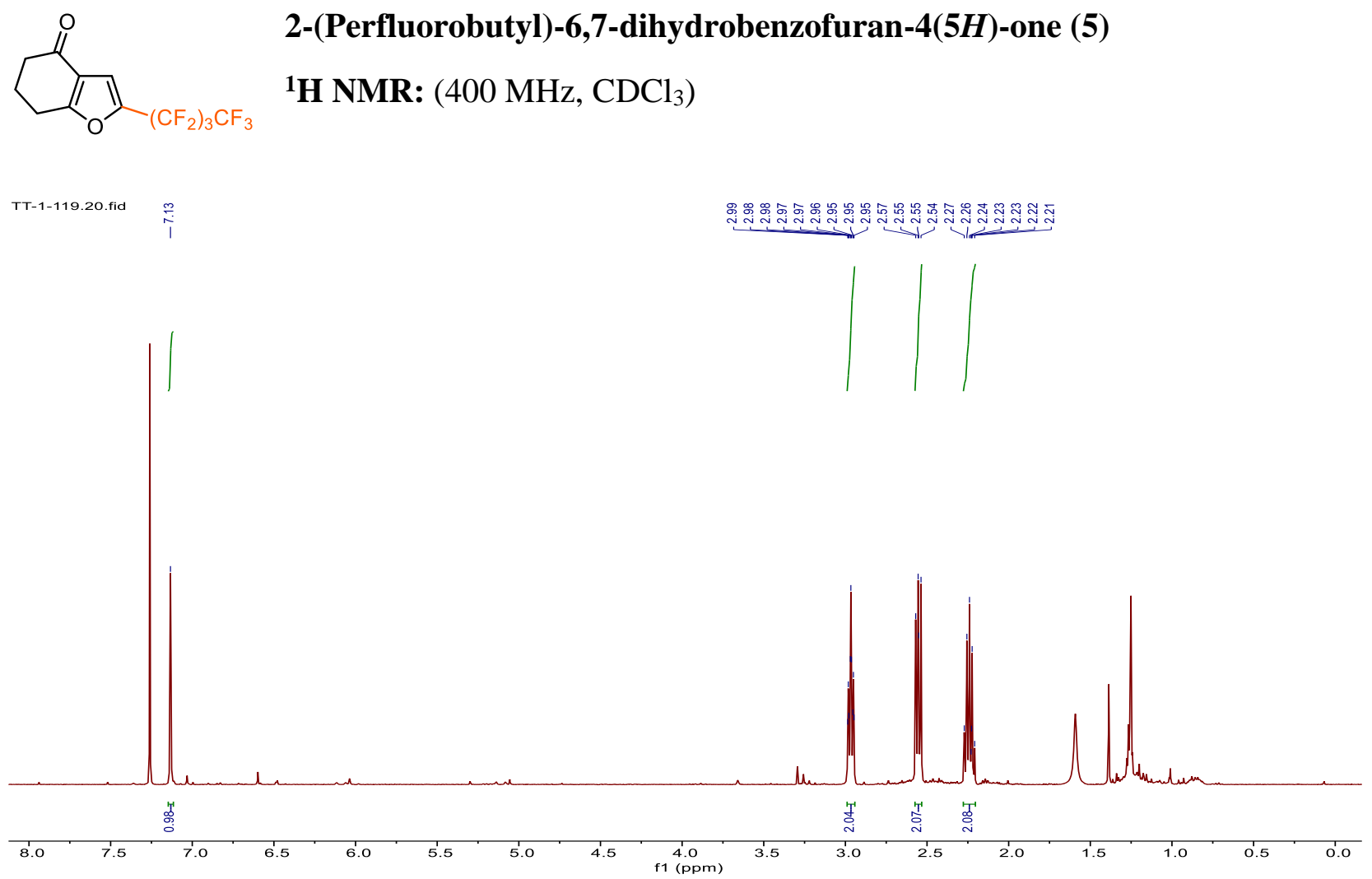

${ }^{13}$ C NMR: $\left(201 \mathrm{MHz}, \mathrm{CDCl}_{3}\right)$

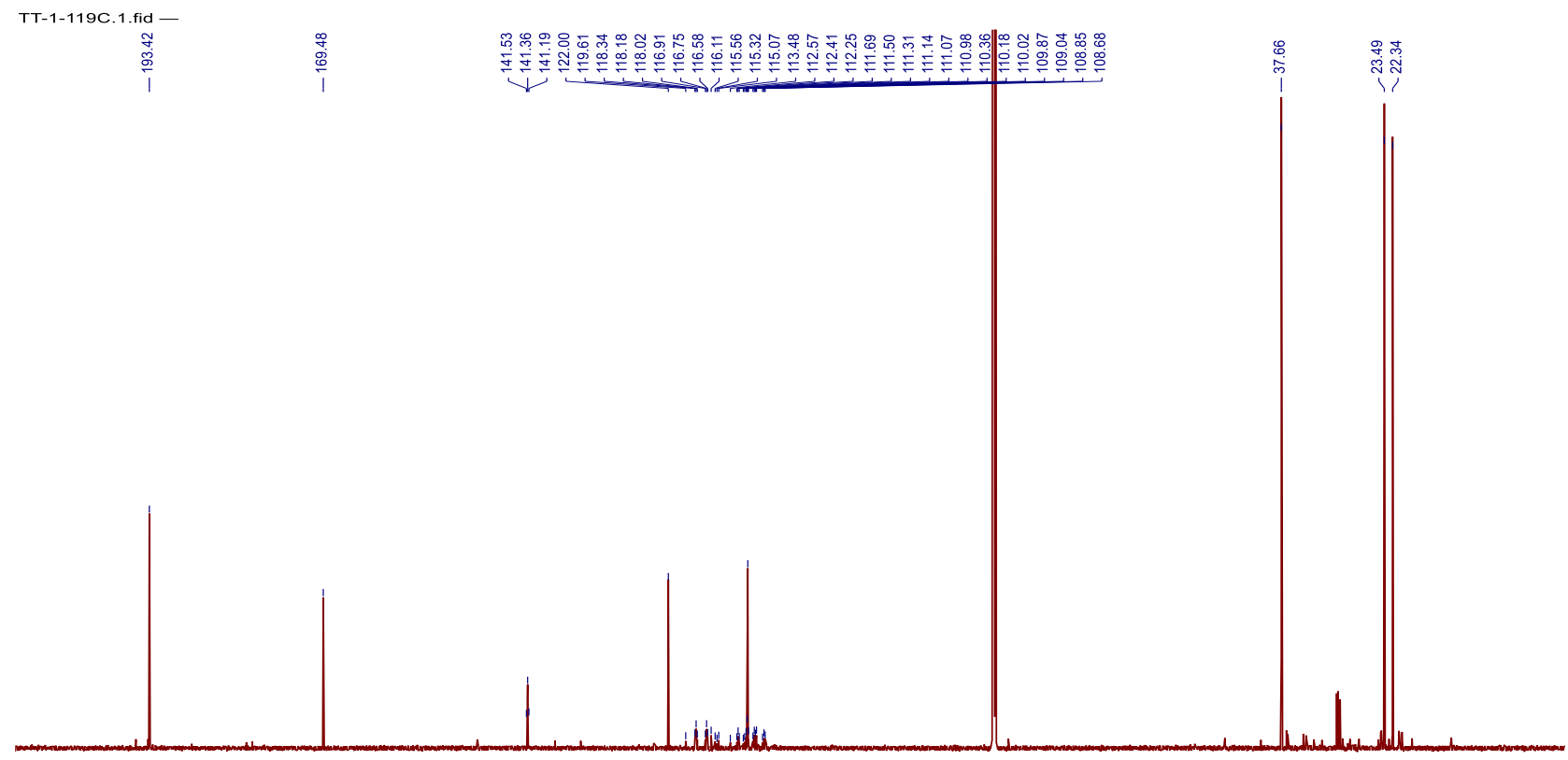

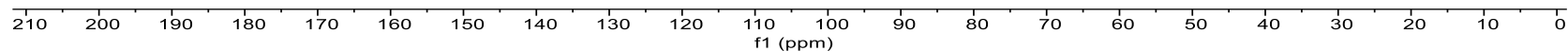




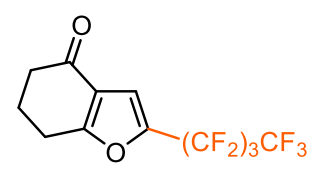

2-(Perfluorobutyl)-6,7-dihydrobenzofuran-4(5H)-one (5)

${ }^{19}$ F NMR: (376 MHz, $\left.\mathrm{CDCl}_{3}\right)$
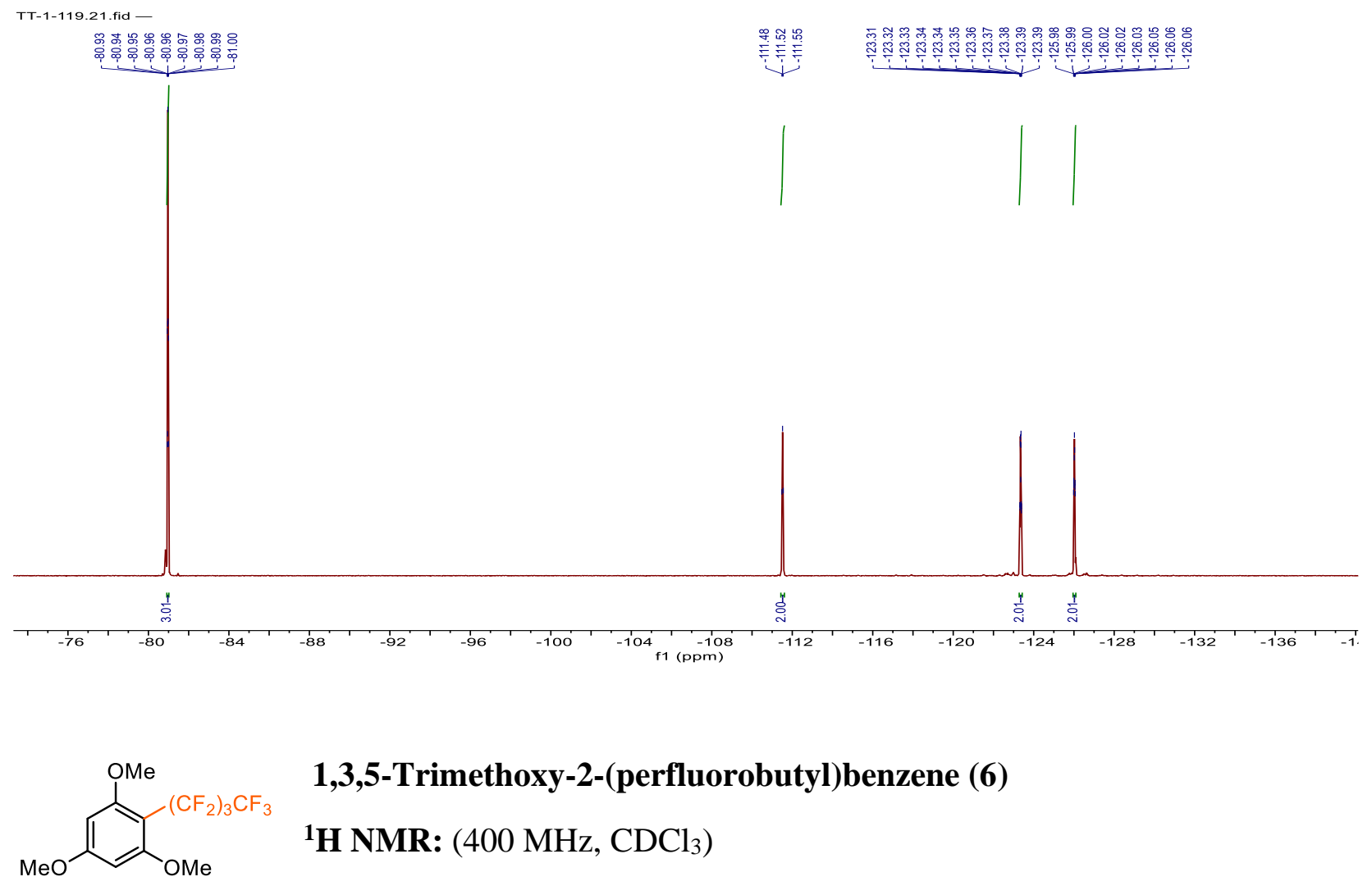

TT_1_69_TMB.10.fid
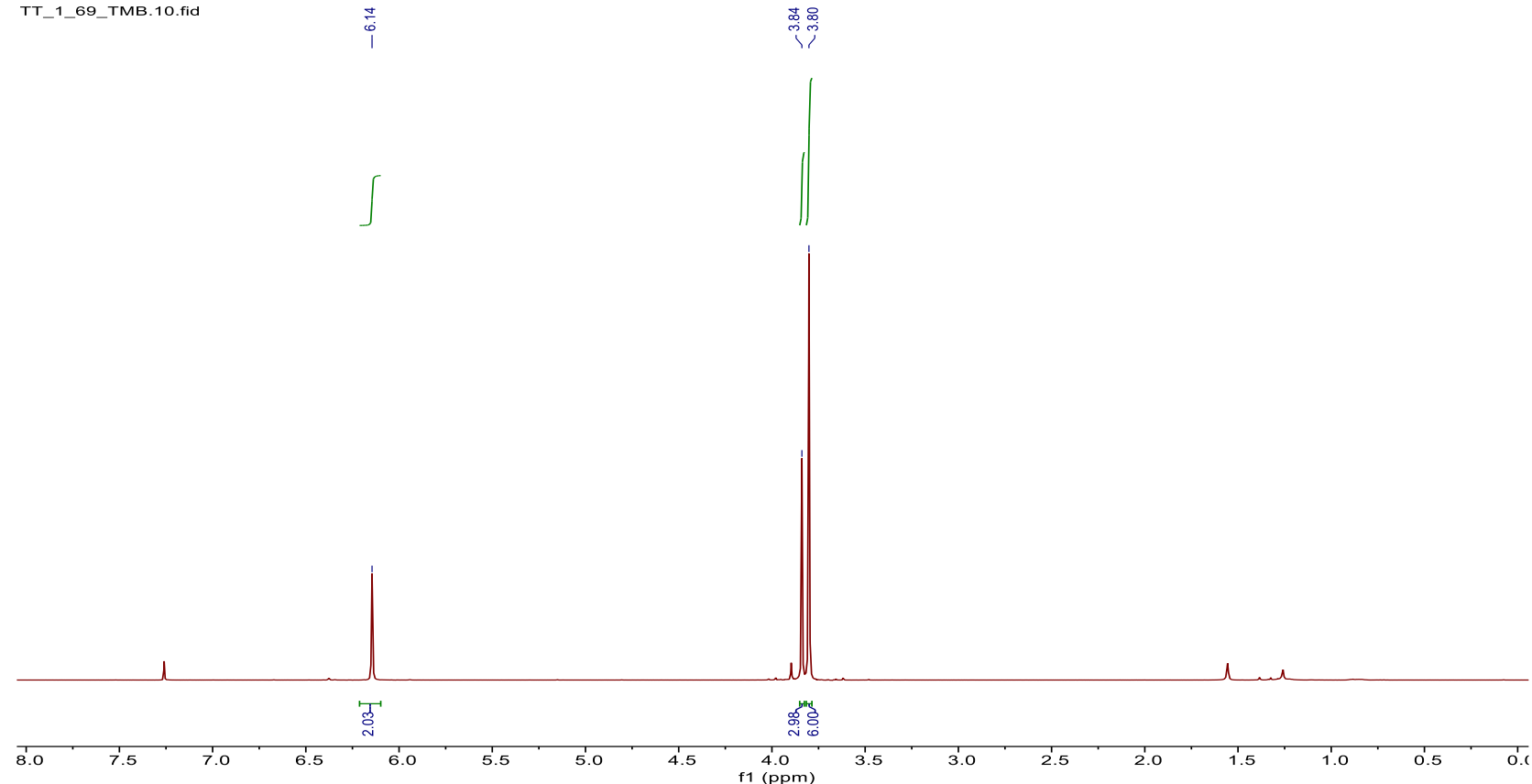
OMe 1,3,5-Trimethoxy-2-(perfluorobutyl)benzene (6)

${ }^{13}$ C NMR: (201 MHz, $\left.\mathrm{CDCl}_{3}\right)$
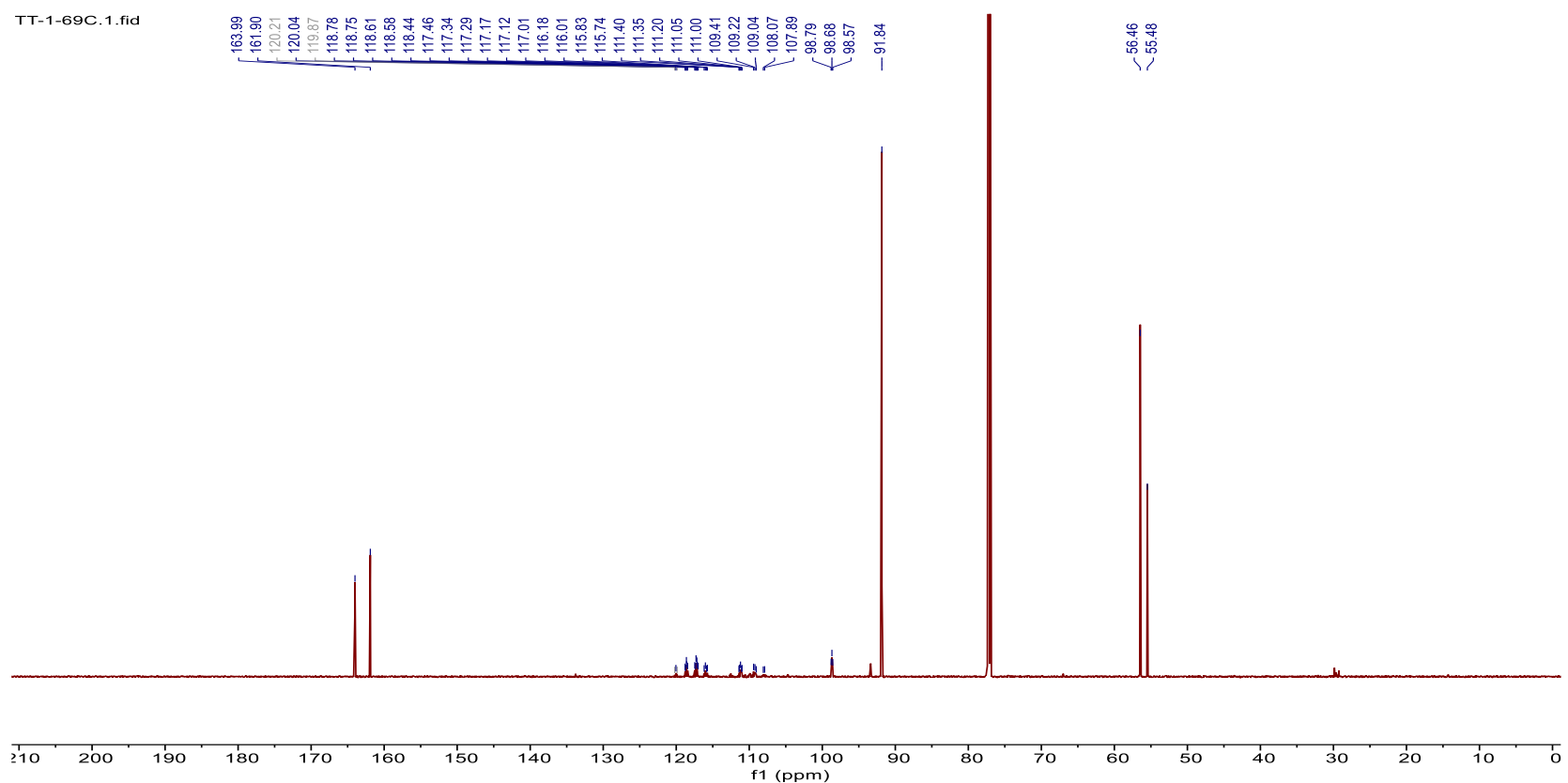

${ }^{19}$ F NMR: $\left(376 \mathrm{MHz}, \mathrm{CDCl}_{3}\right)$

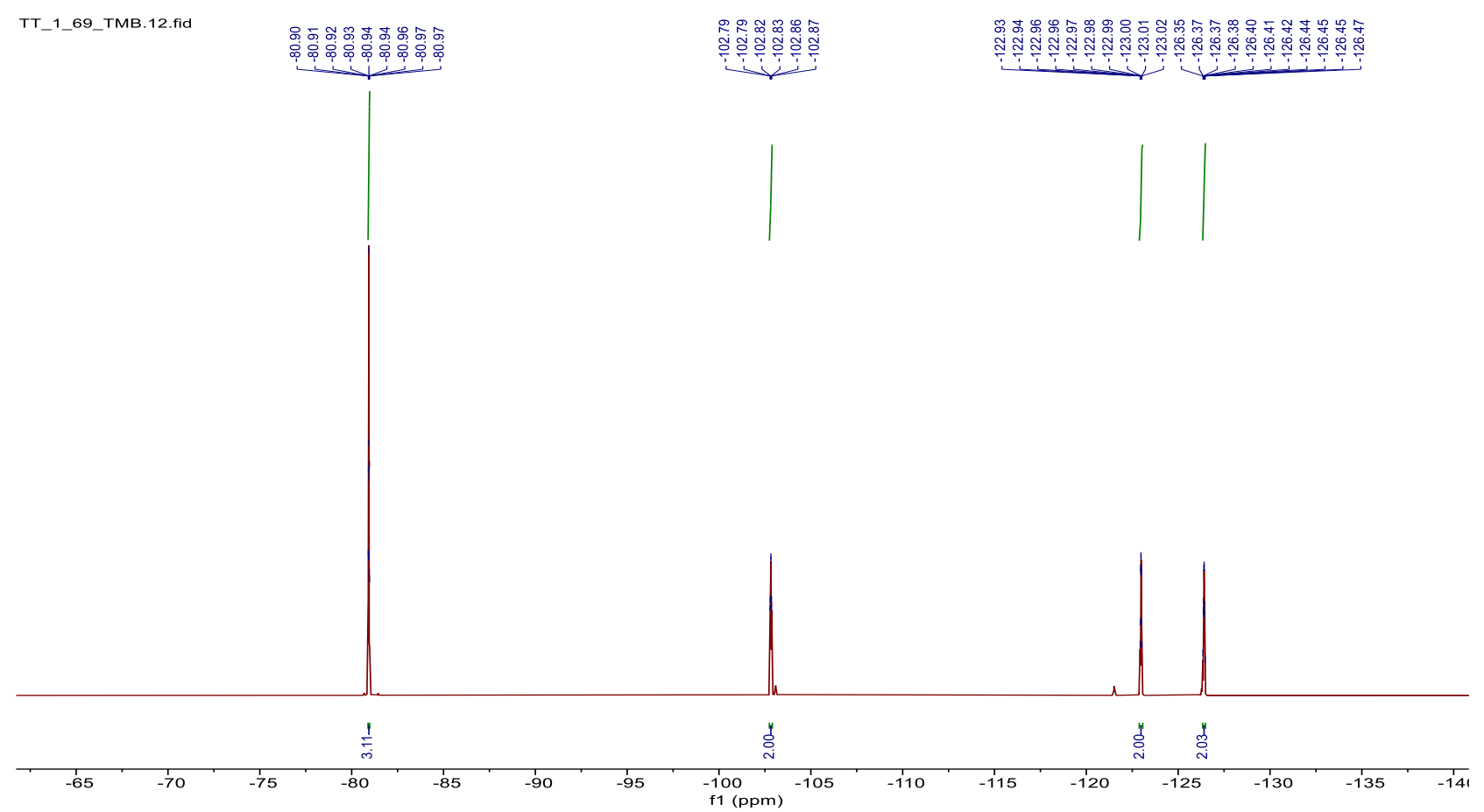



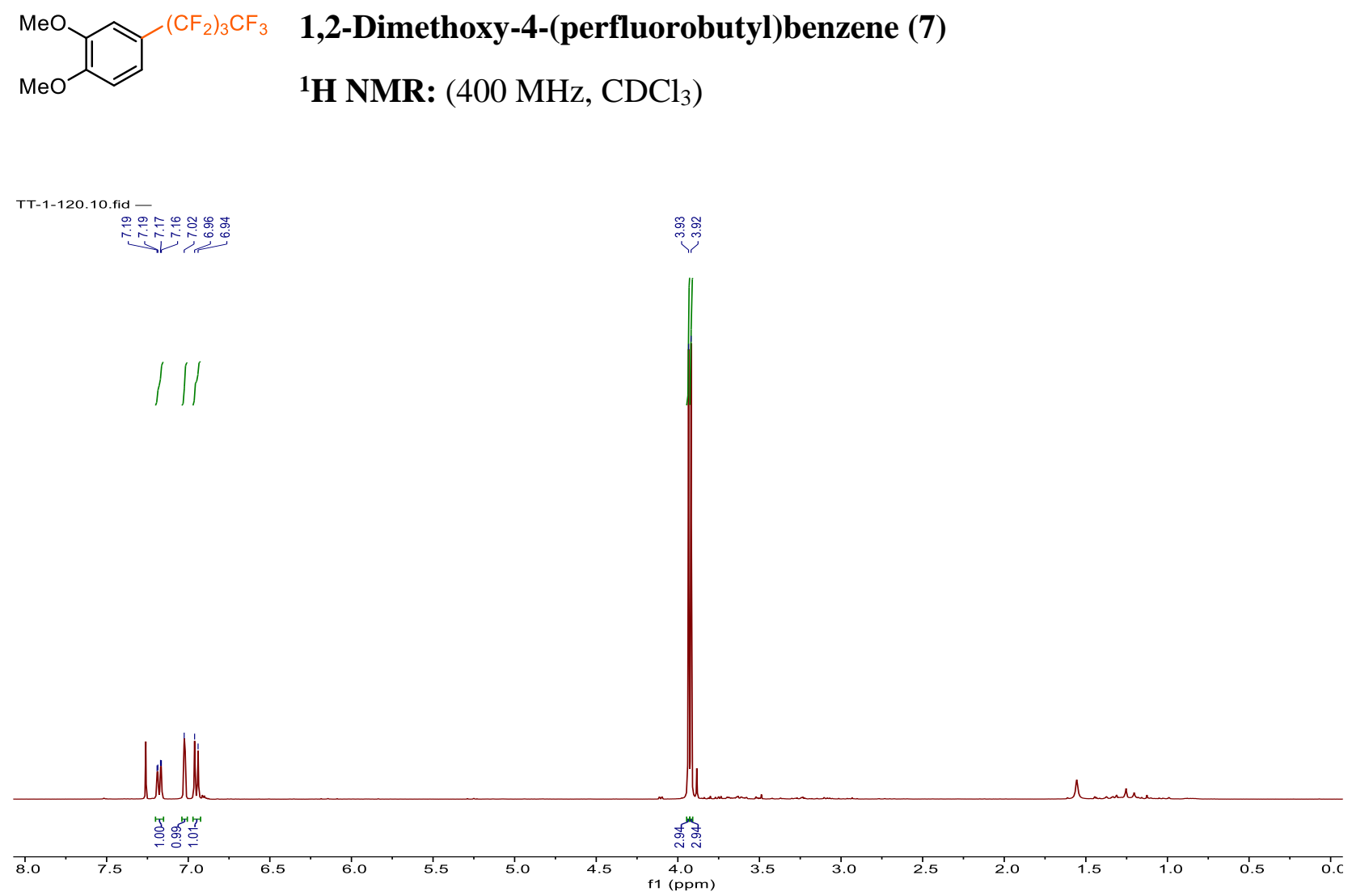

${ }^{13}$ C NMR: (201 MHz, $\left.\mathrm{CDCl}_{3}\right)$

TT-1-120C.1.fid

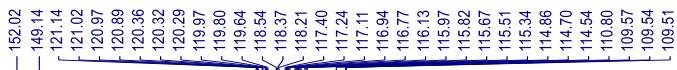
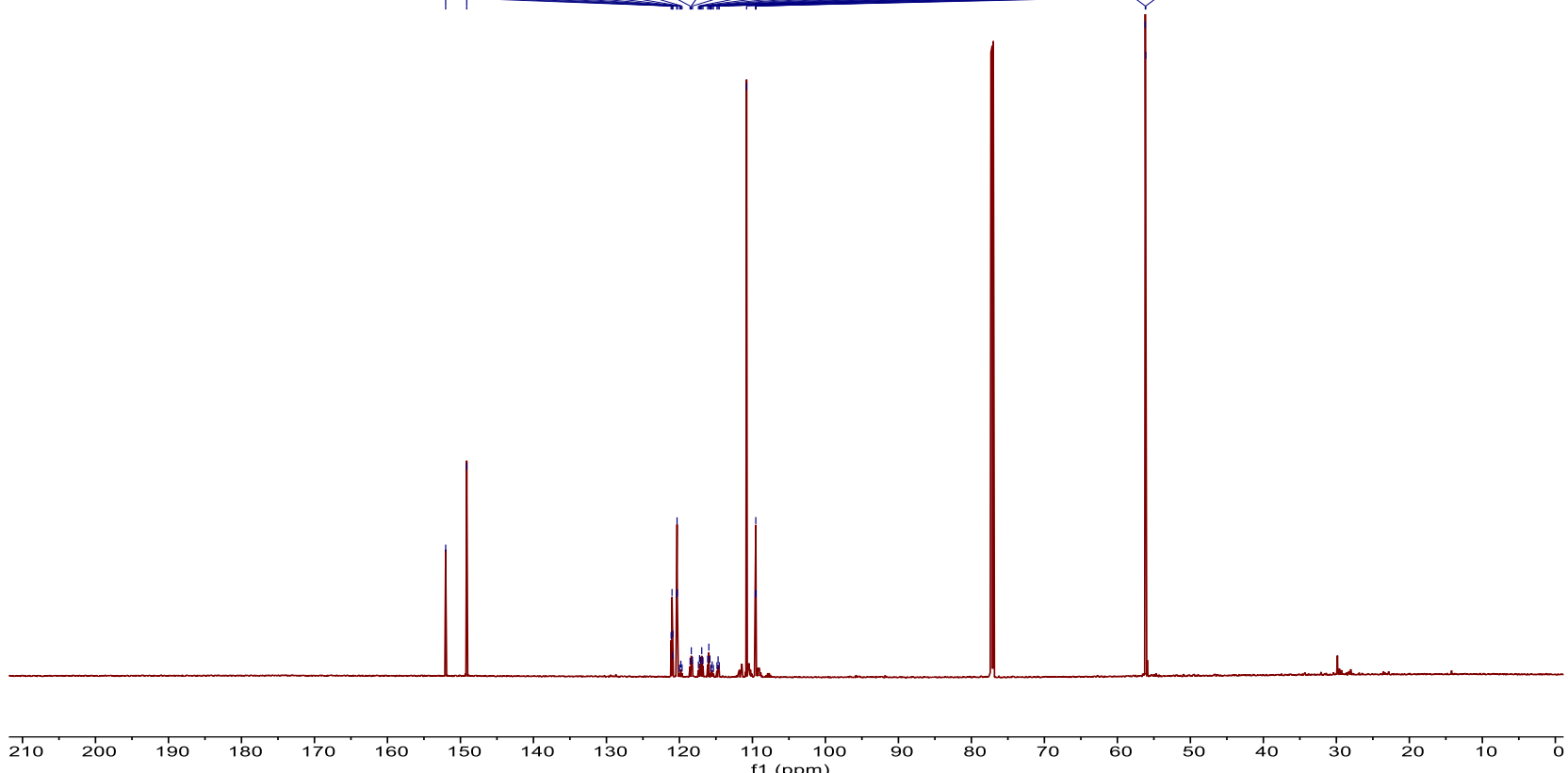
$\left(\mathrm{CF}_{2}\right)_{3} \mathrm{CF}_{3}$

TT-1-120.13.fid -

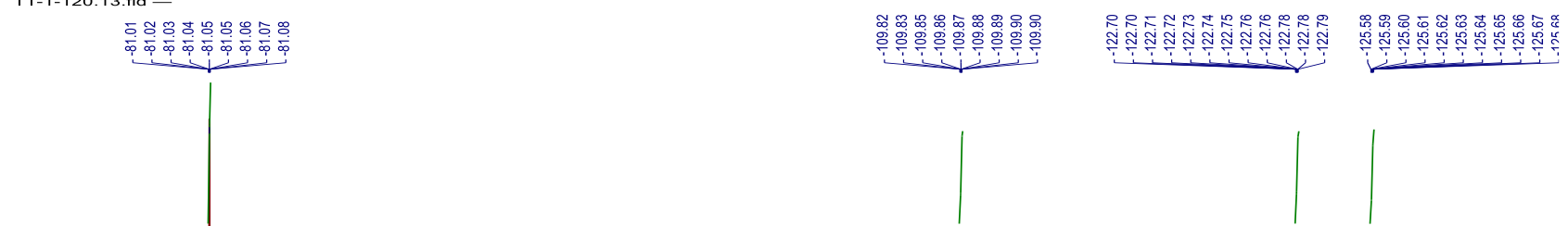

${ }^{19}$ F NMR: (376 MHz, $\mathrm{CDCl}_{3}$ )

\section{1,2-Dimethoxy-4-(perfluorobutyl)benzene (7)}




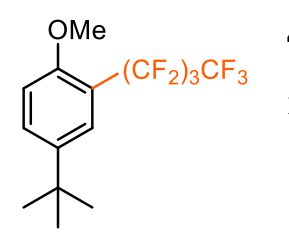

TT-1-78C.1.fid

4-(Tert-butyl)-1-methoxy-2-(perfluorobutyl)benzene (8)

${ }^{13}$ C NMR: (201 MHz, $\left.\mathrm{CDCl}_{3}\right)$

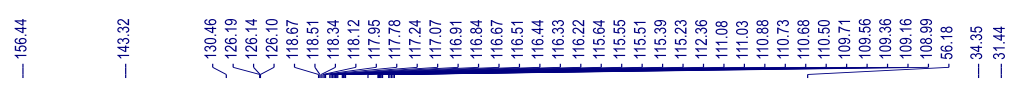

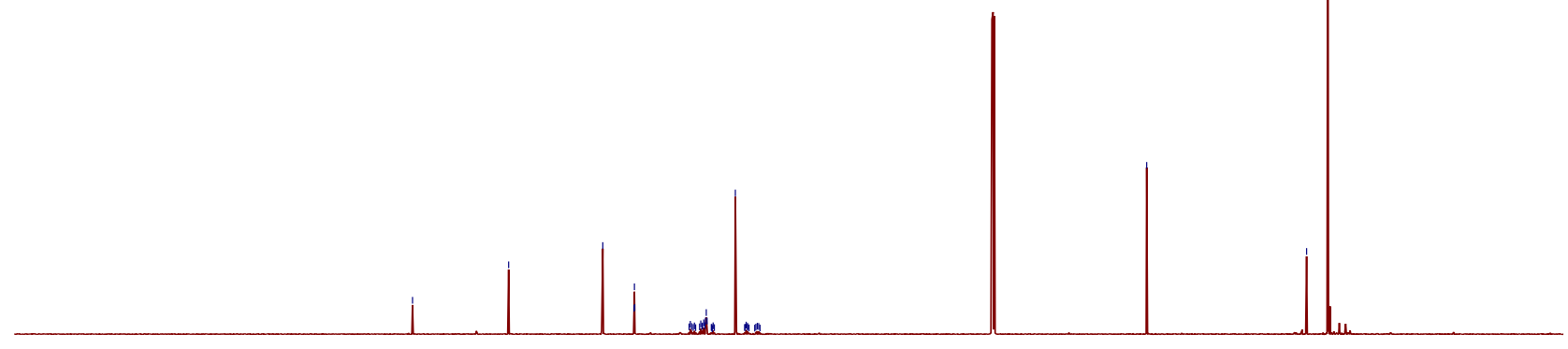

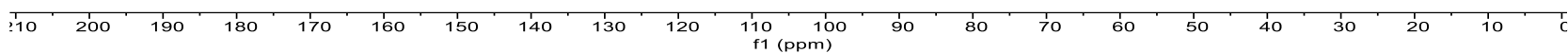

${ }^{19}$ F NMR: $\left(376 \mathrm{MHz}, \mathrm{CDCl}_{3}\right)$

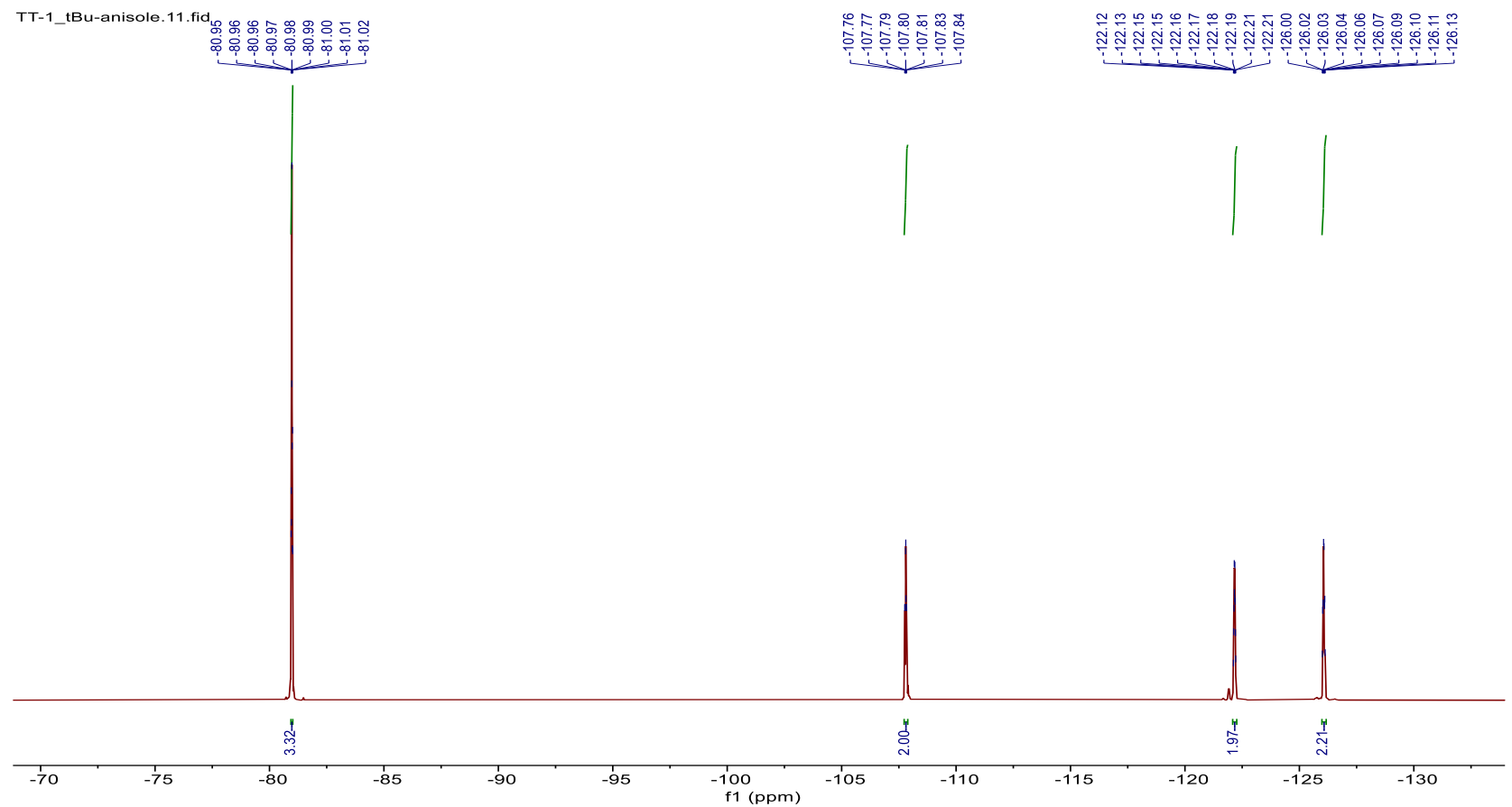




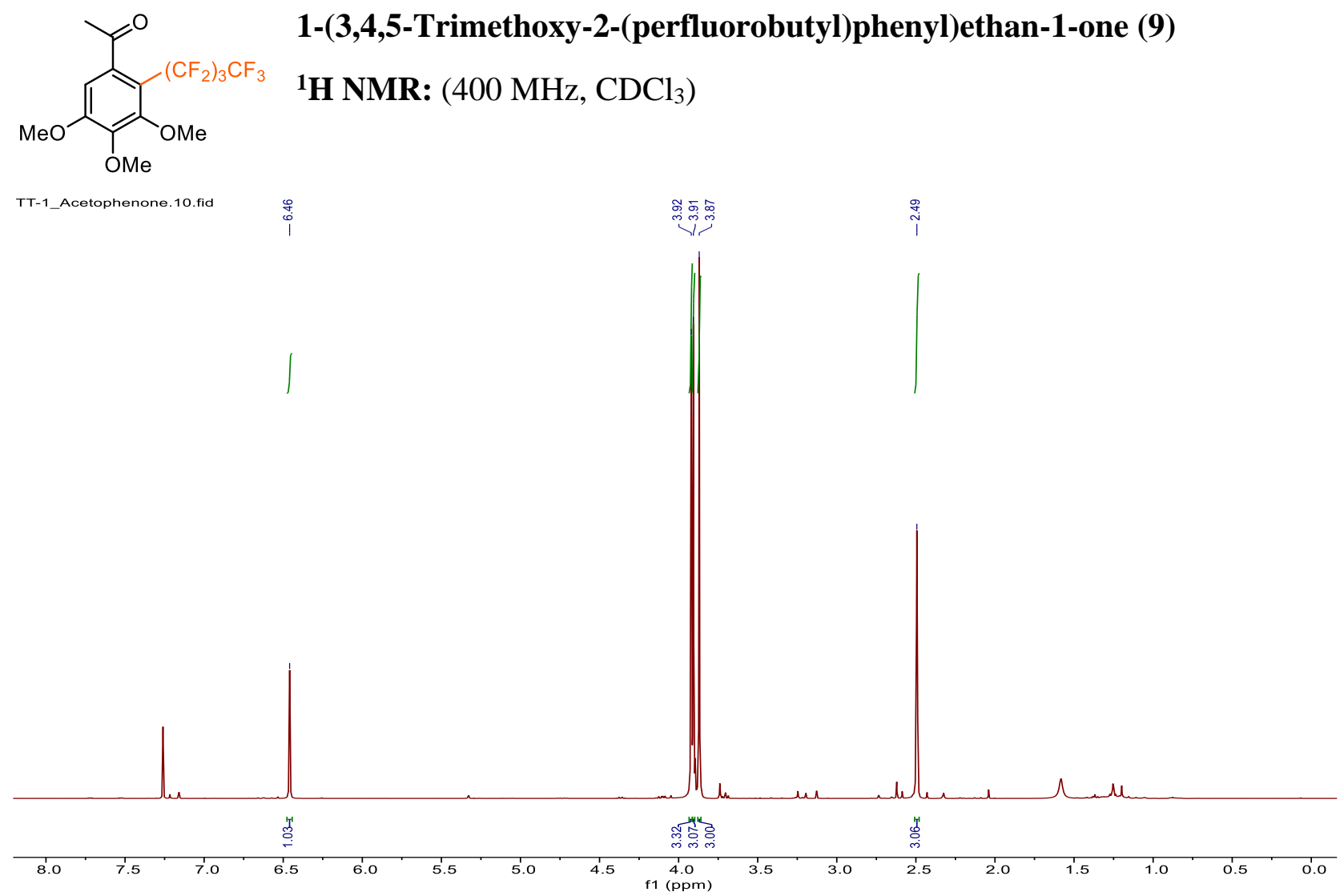

${ }^{13}$ C NMR: (201 MHz, $\mathrm{CDCl}_{3}$ )

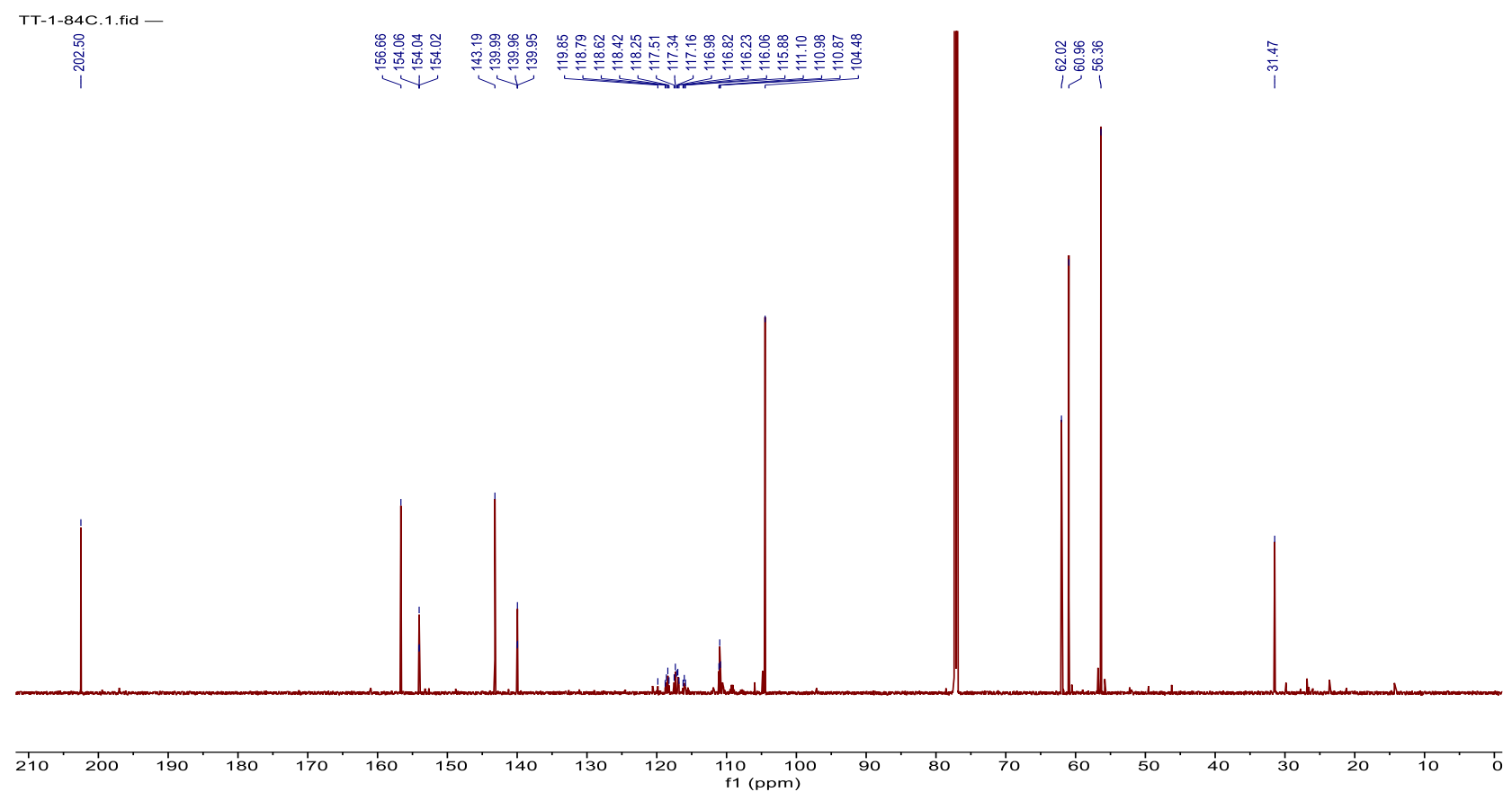




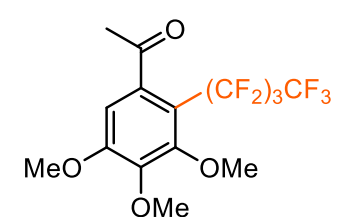

1-(3,4,5-Trimethoxy-2-(perfluorobutyl)phenyl)ethan-1-one (9)

${ }^{19}$ F NMR: $\left(376 \mathrm{MHz}, \mathrm{CDCl}_{3}\right)$
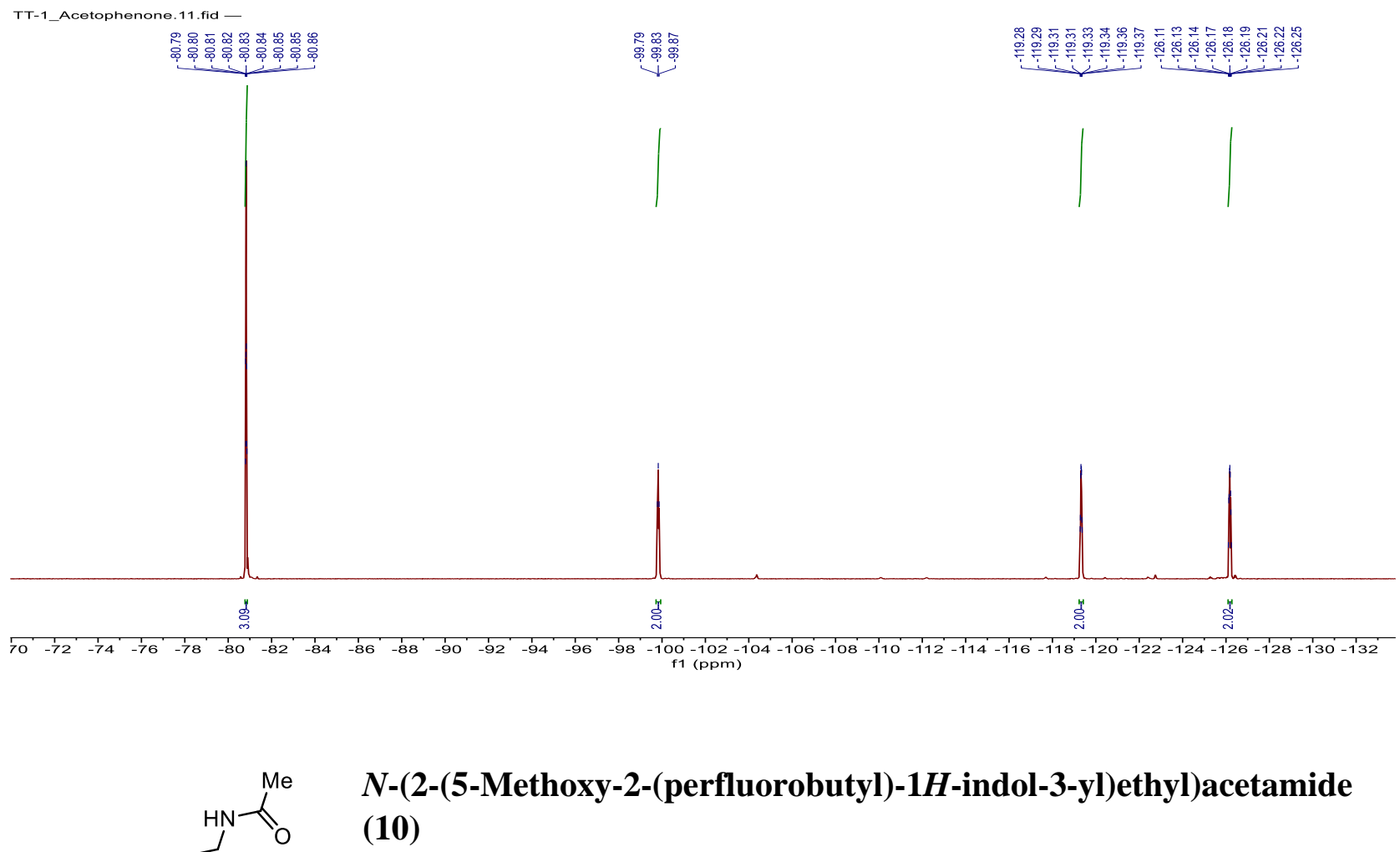

${ }^{1}$ H NMR: (400 MHz, $\mathrm{CDCl}_{3}$ )

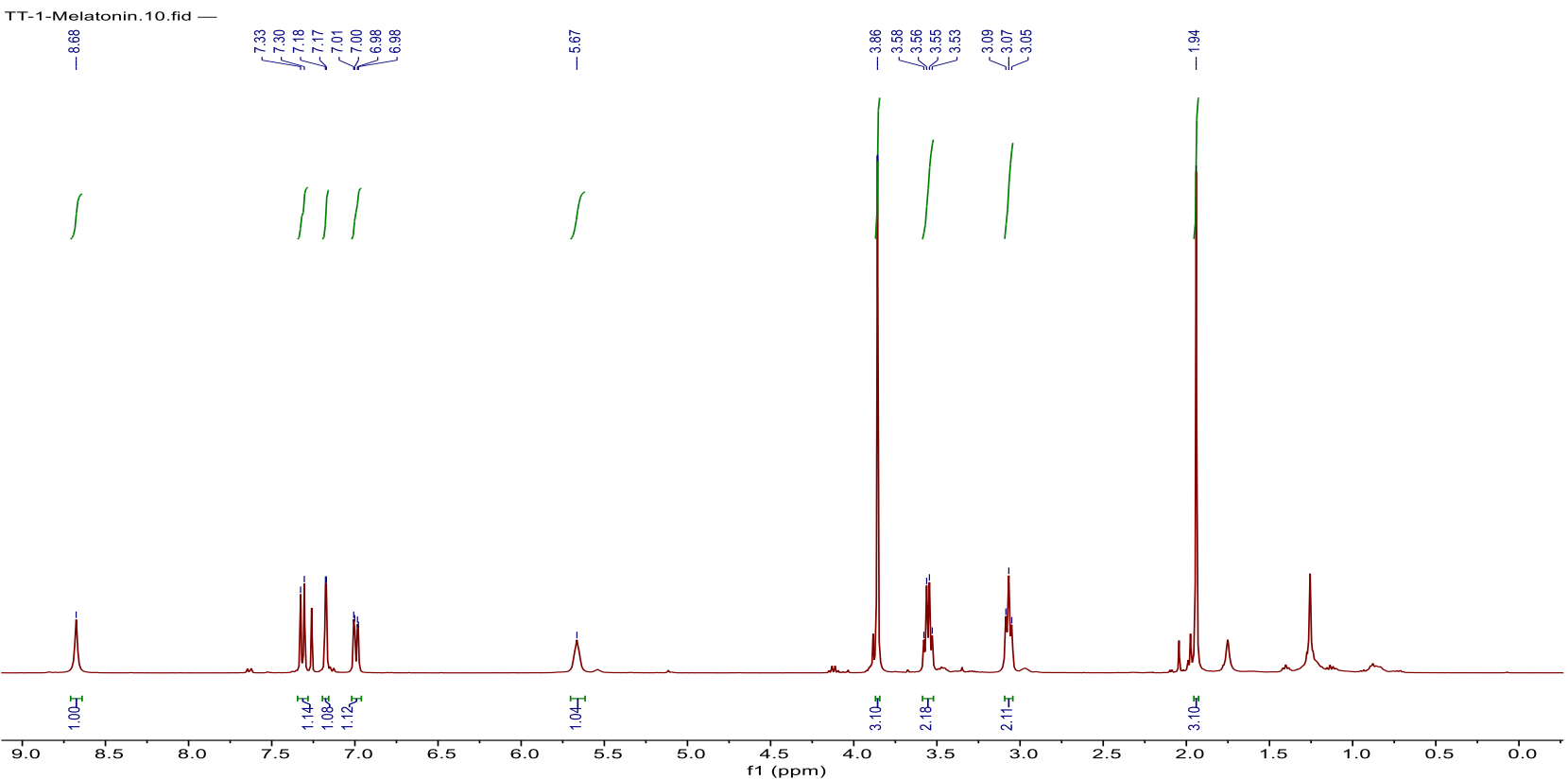




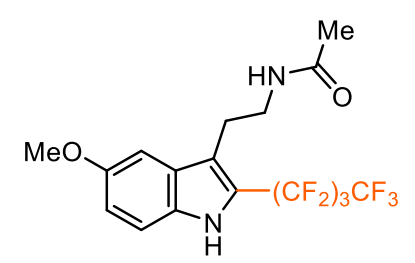

$N$-(2-(5-Methoxy-2-(perfluorobutyl)-1H-indol-3-yl)ethyl)acetamide

(10)

${ }^{13}$ C NMR: (201 MHz, $\left.\mathrm{CDCl}_{3}\right)$

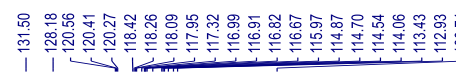

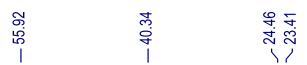
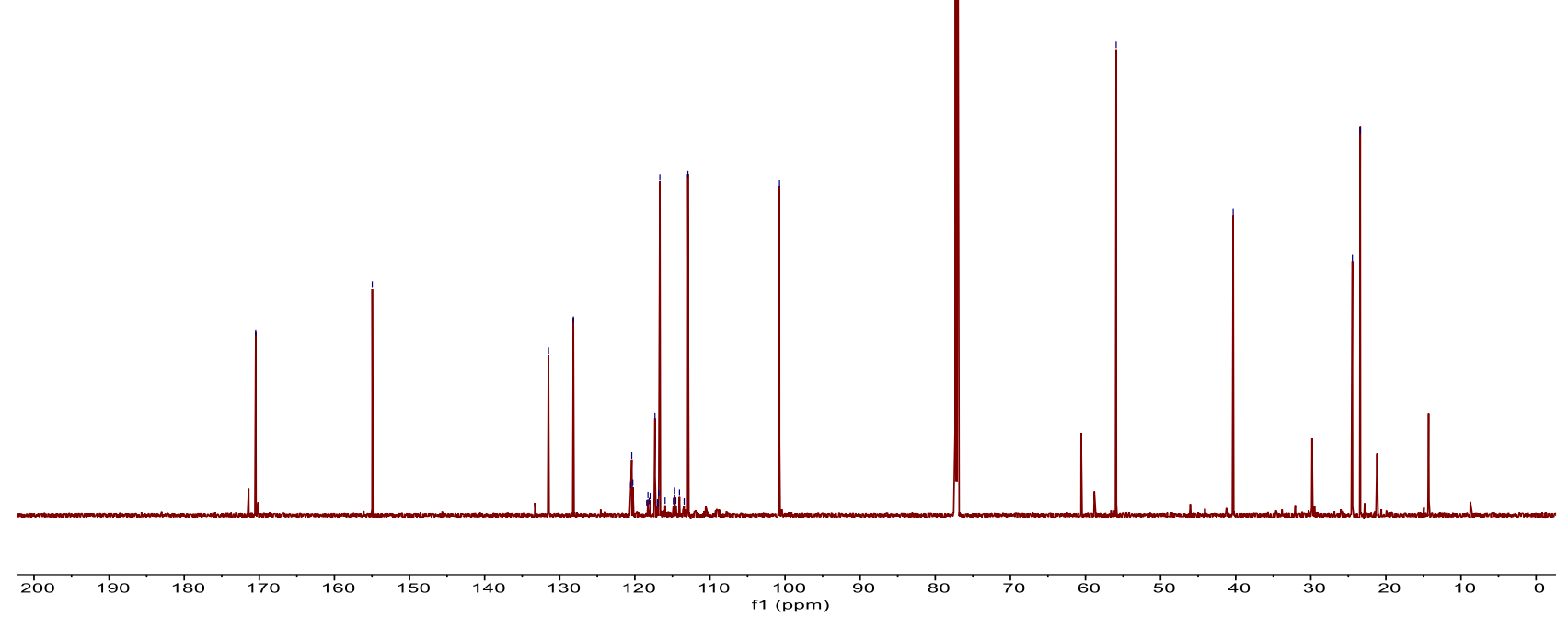

19F NMR: (376 MHz, $\mathrm{CDCl}_{3}$ )

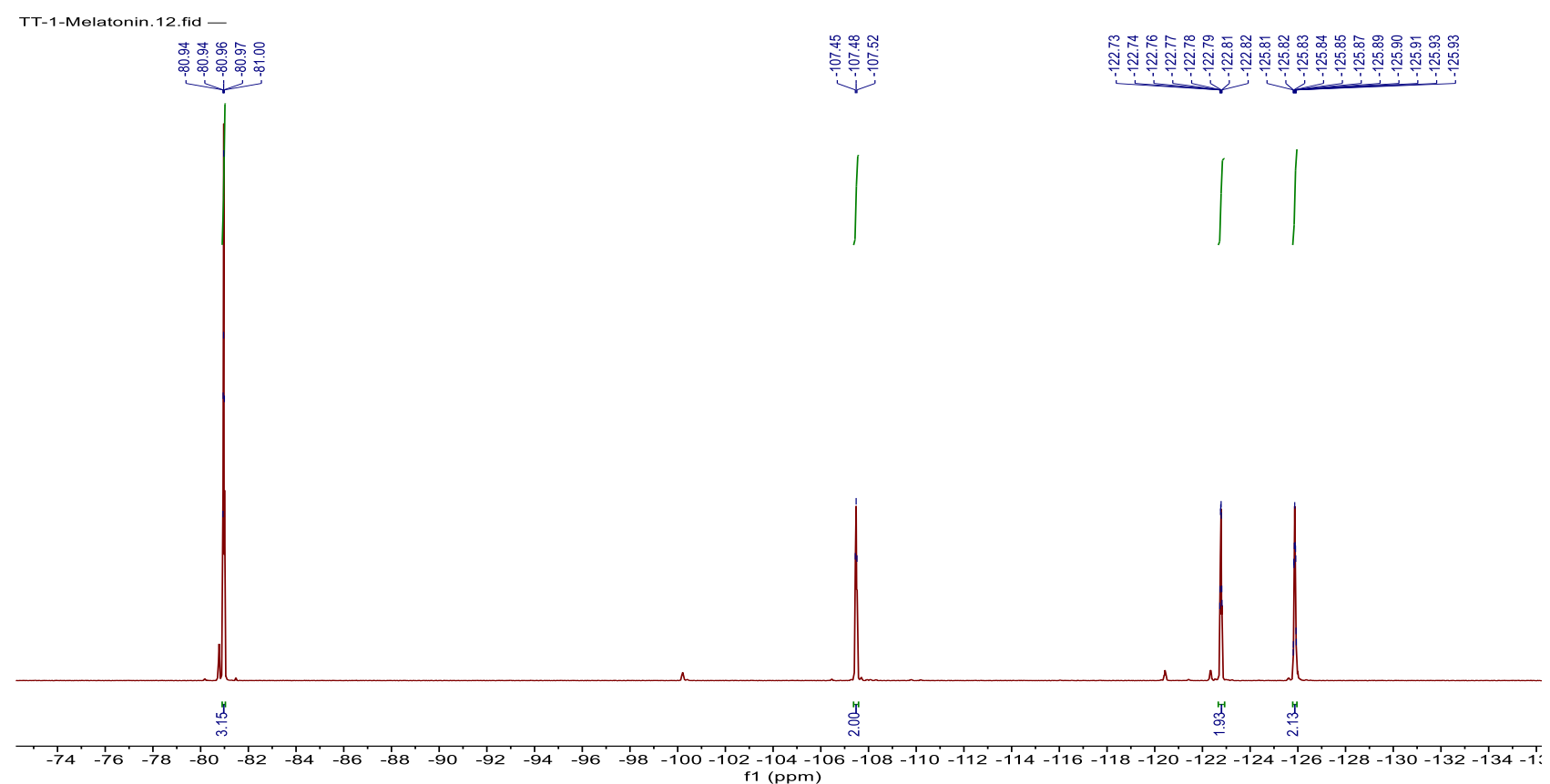




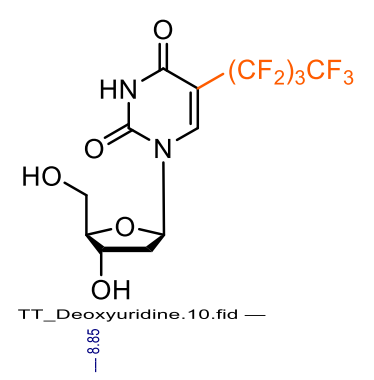

1-((2R,4S,5R)-4-Hydroxy-5-(hydroxymethyl)tetrahydrofuran-2-yl)-5(perfluorobutyl)pyrimidine-2,4(1H,3H)-dione (11)

${ }^{1} \mathbf{H}$ NMR: (400 MHz, $\left.\mathrm{CD}_{3} \mathrm{OD}\right)$

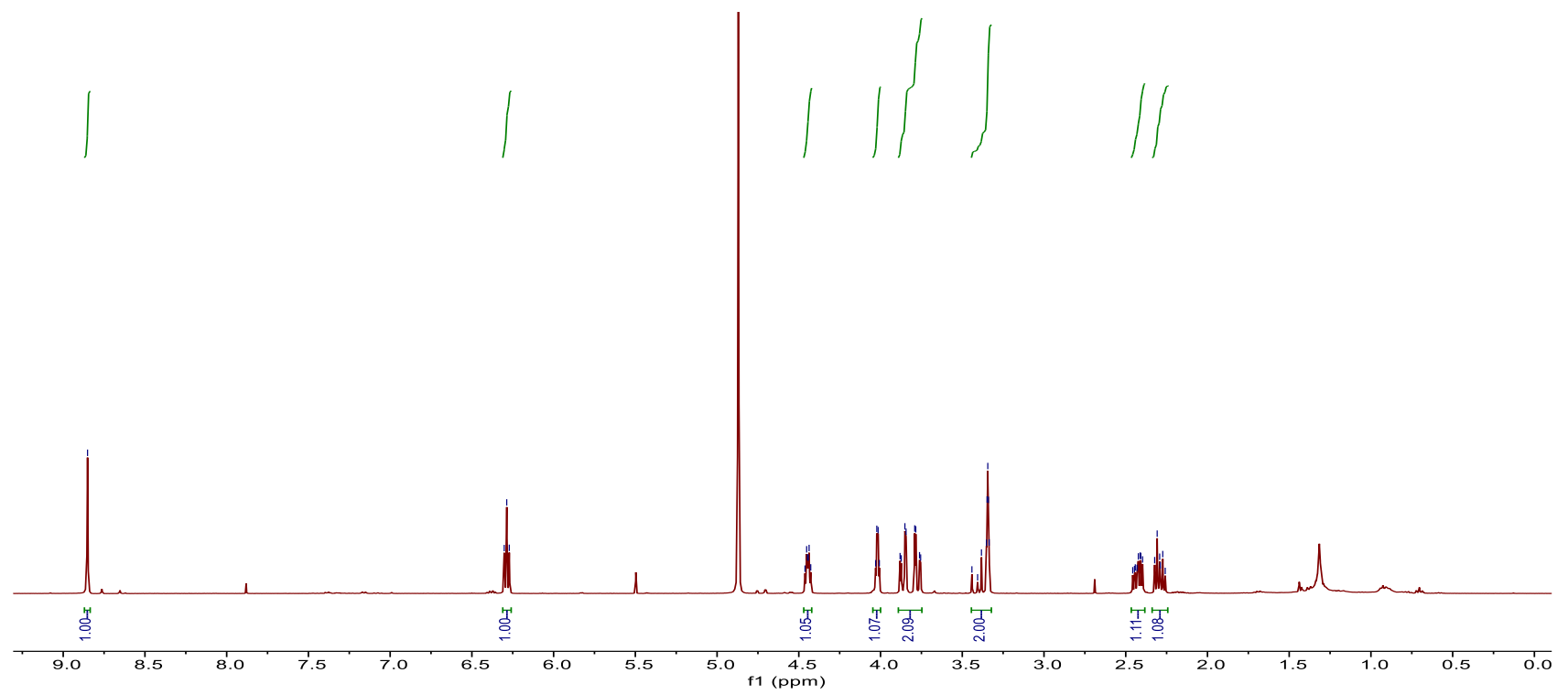

${ }^{13}$ C NMR: (201 MHz, $\left.\mathrm{CD}_{3} \mathrm{OD}\right)$
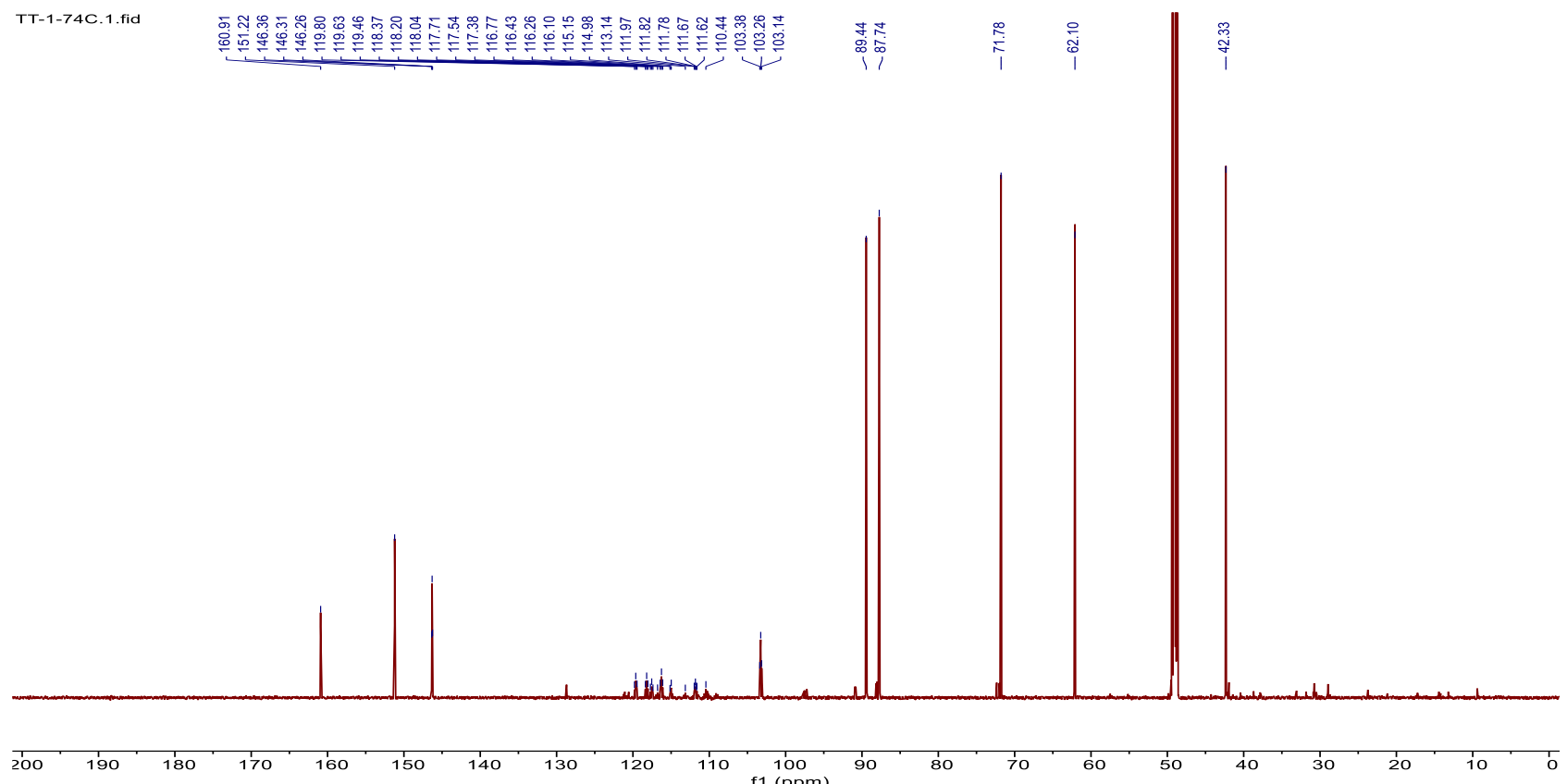


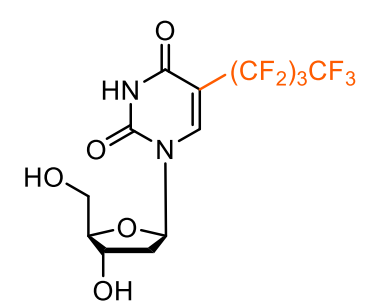

TT_Deoxyuridine 11. fid -
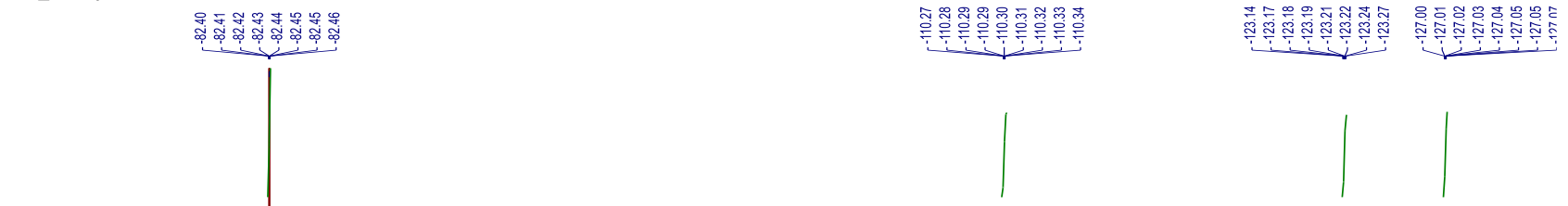

1-((2R,4S,5R)-4-Hydroxy-5-(hydroxymethyl)tetrahydrofuran-2-yl)-5(perfluorobutyl)pyrimidine-2,4(1H,3H)-dione (11)

${ }^{19}$ F NMR: $\left(376 \mathrm{MHz}, \mathrm{CD}_{3} \mathrm{OD}\right)$
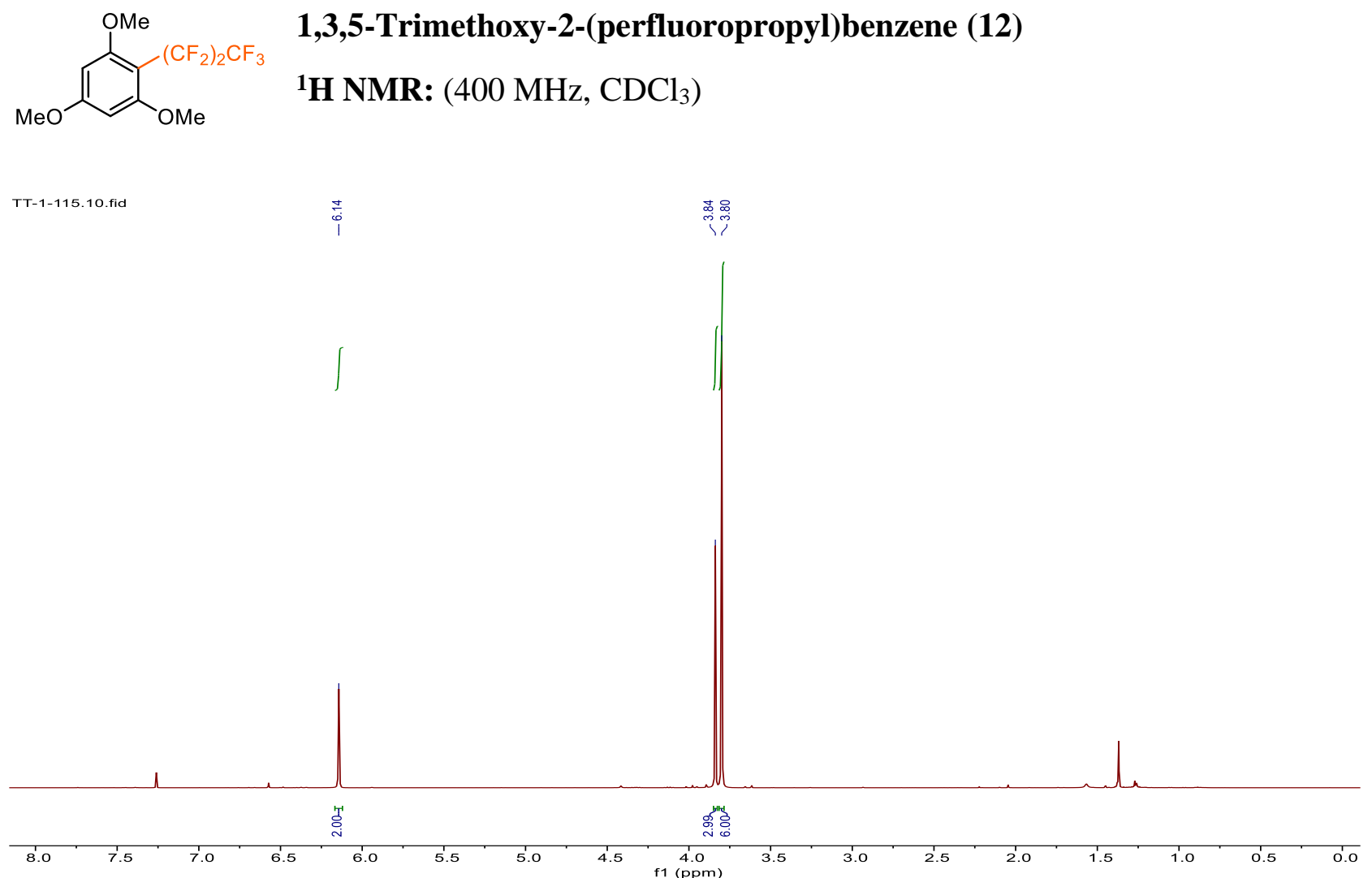
<smiles>COc1cc(OC)c(C(F)(C(F)(F)F)C(F)(F)F)c(OC)c1</smiles>

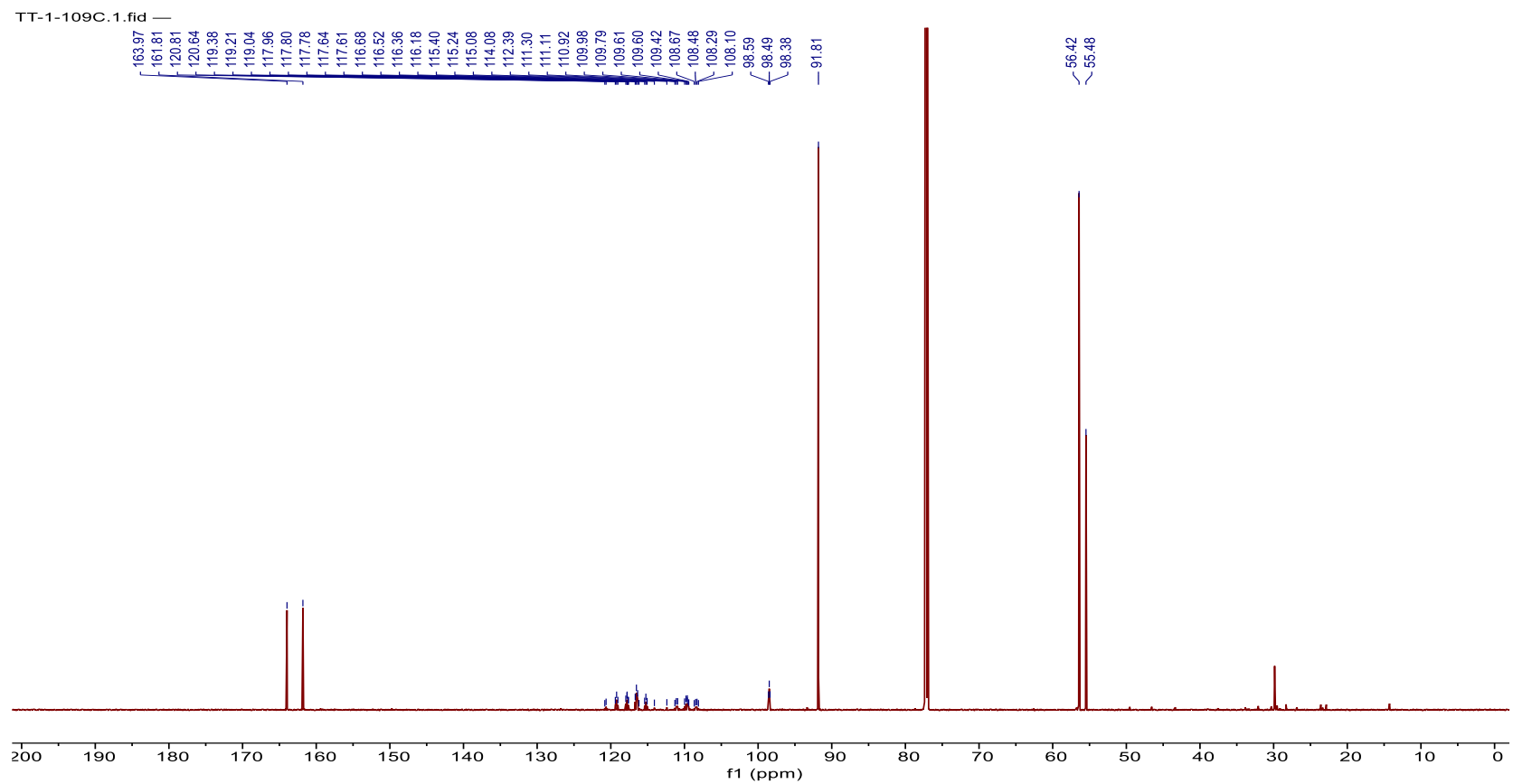

${ }^{19}$ F NMR: (376 MHz, $\mathrm{CDCl}_{3}$ )

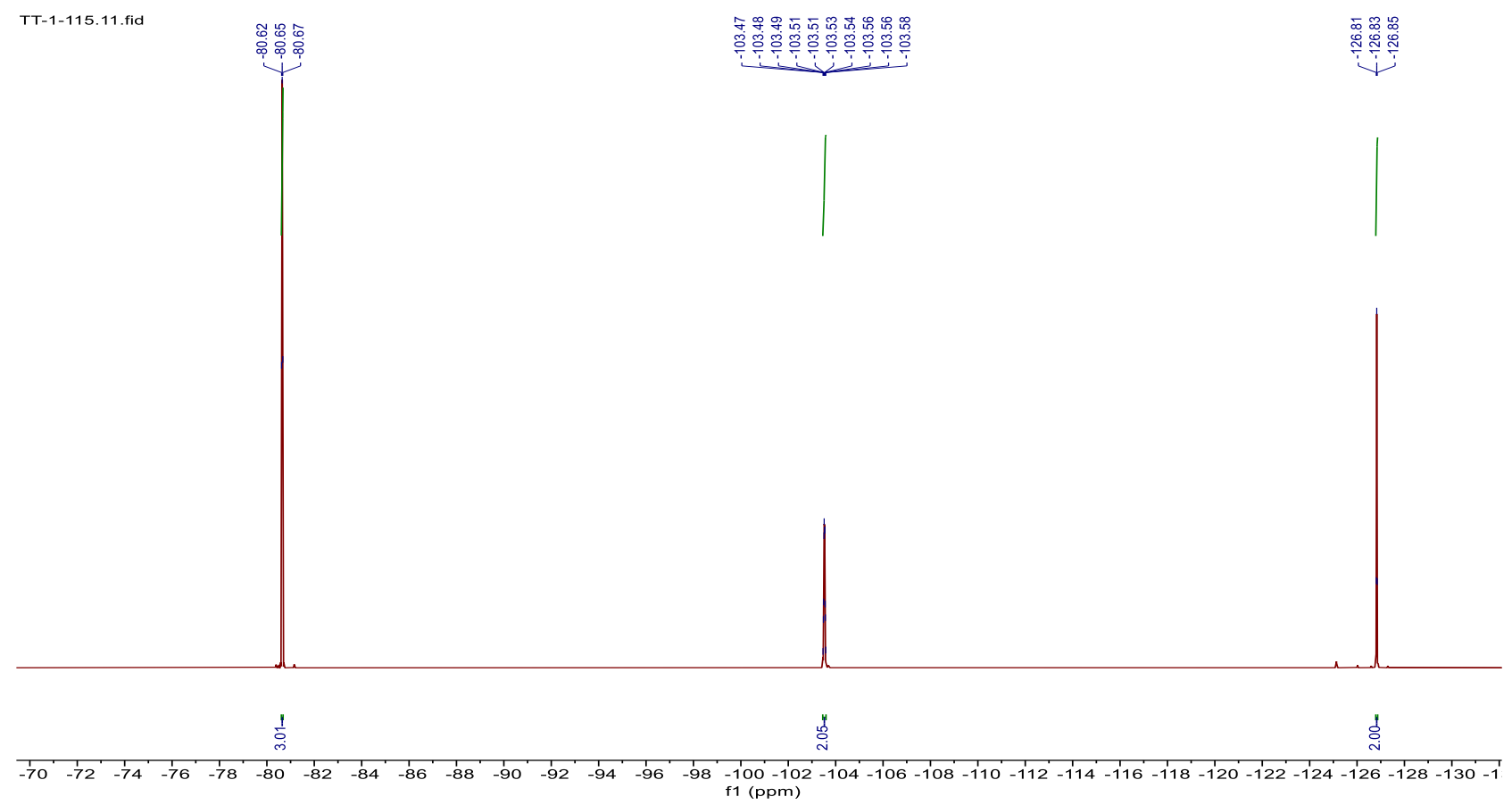



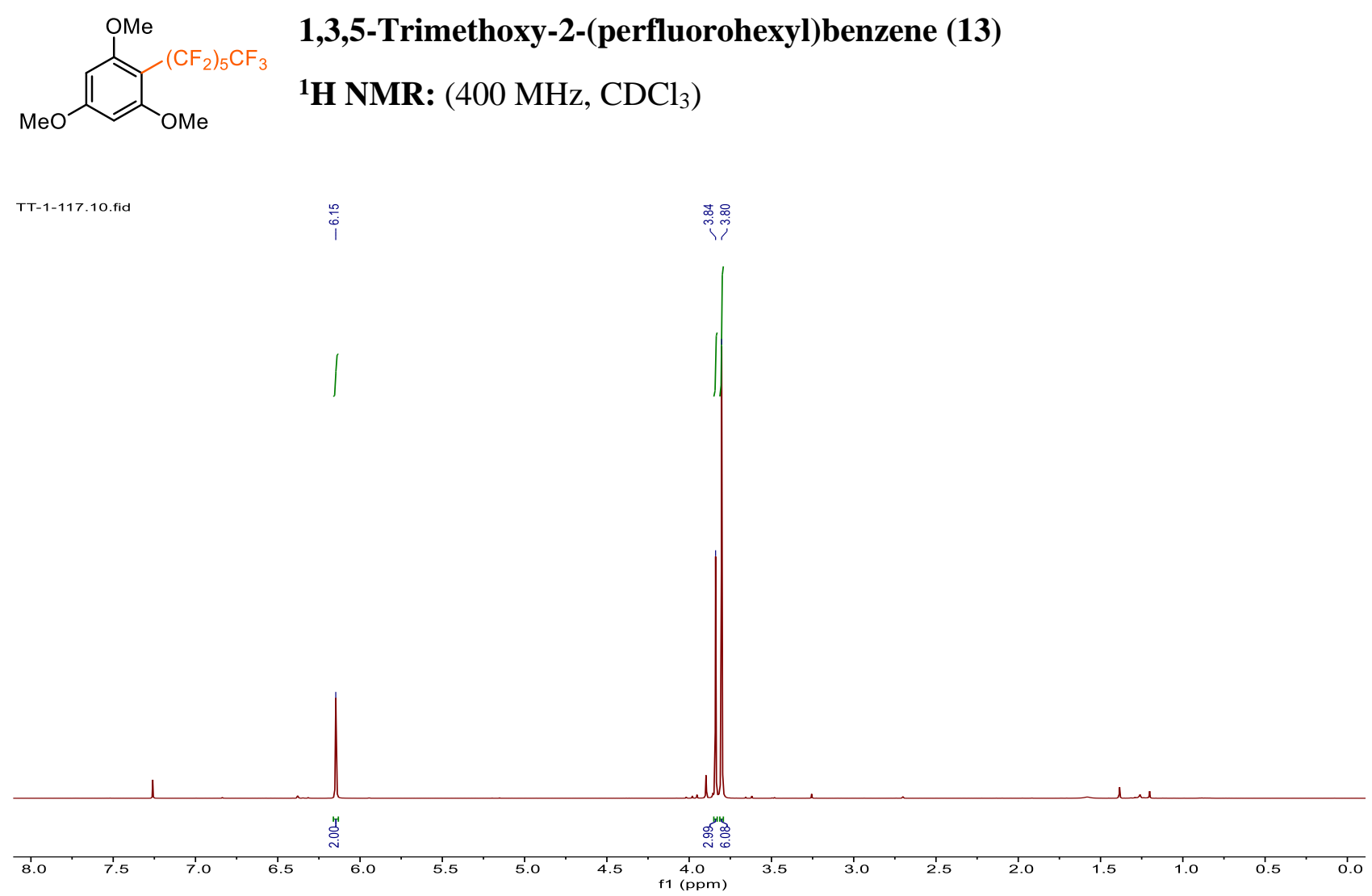

${ }^{13}$ C NMR: (201 MHz, $\mathrm{CDCl}_{3}$ )
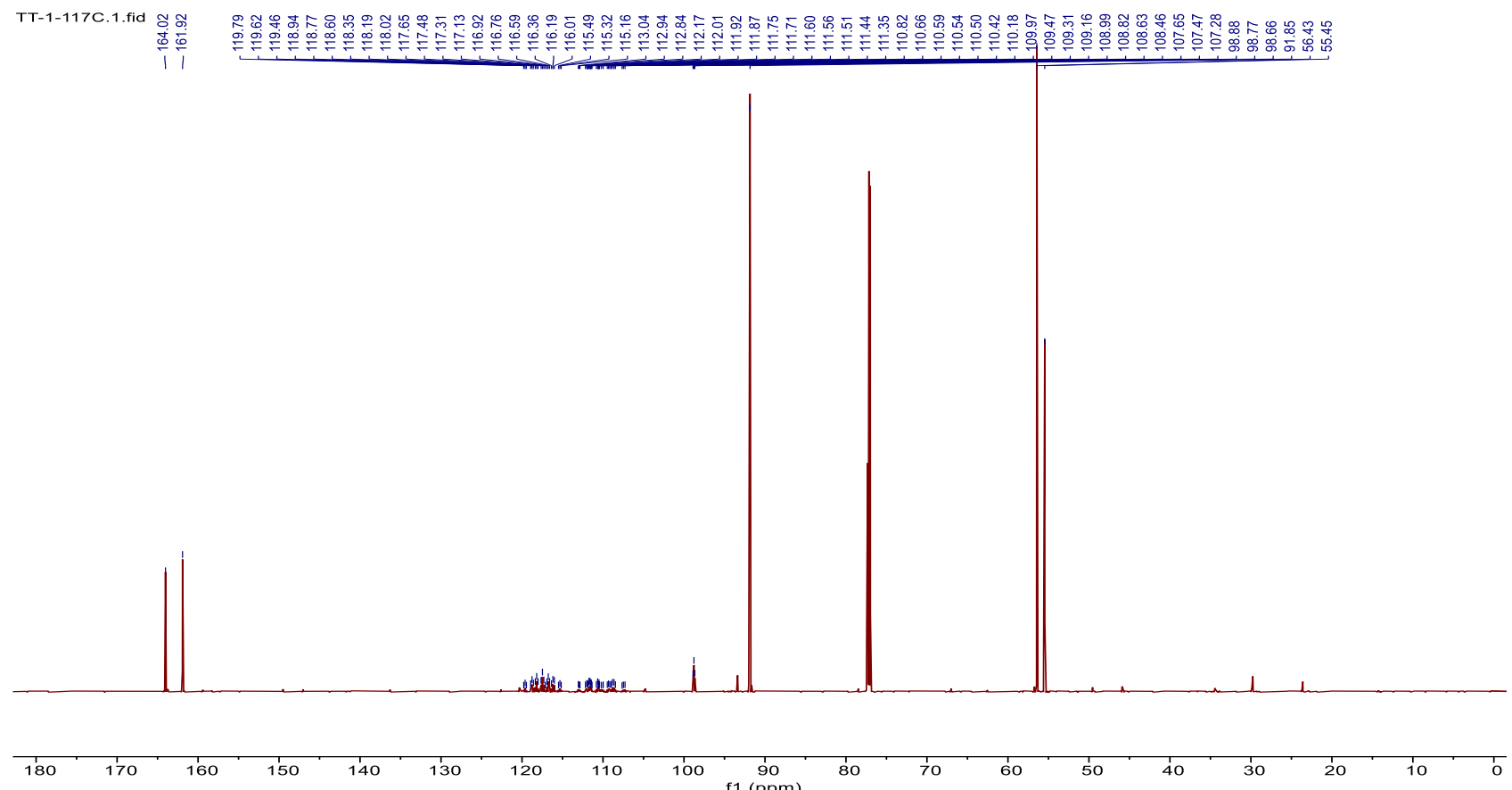
<smiles>COc1cc(OC)c(C(F)(F)F)c(OC)c1</smiles>

TT-1-117.11.fid
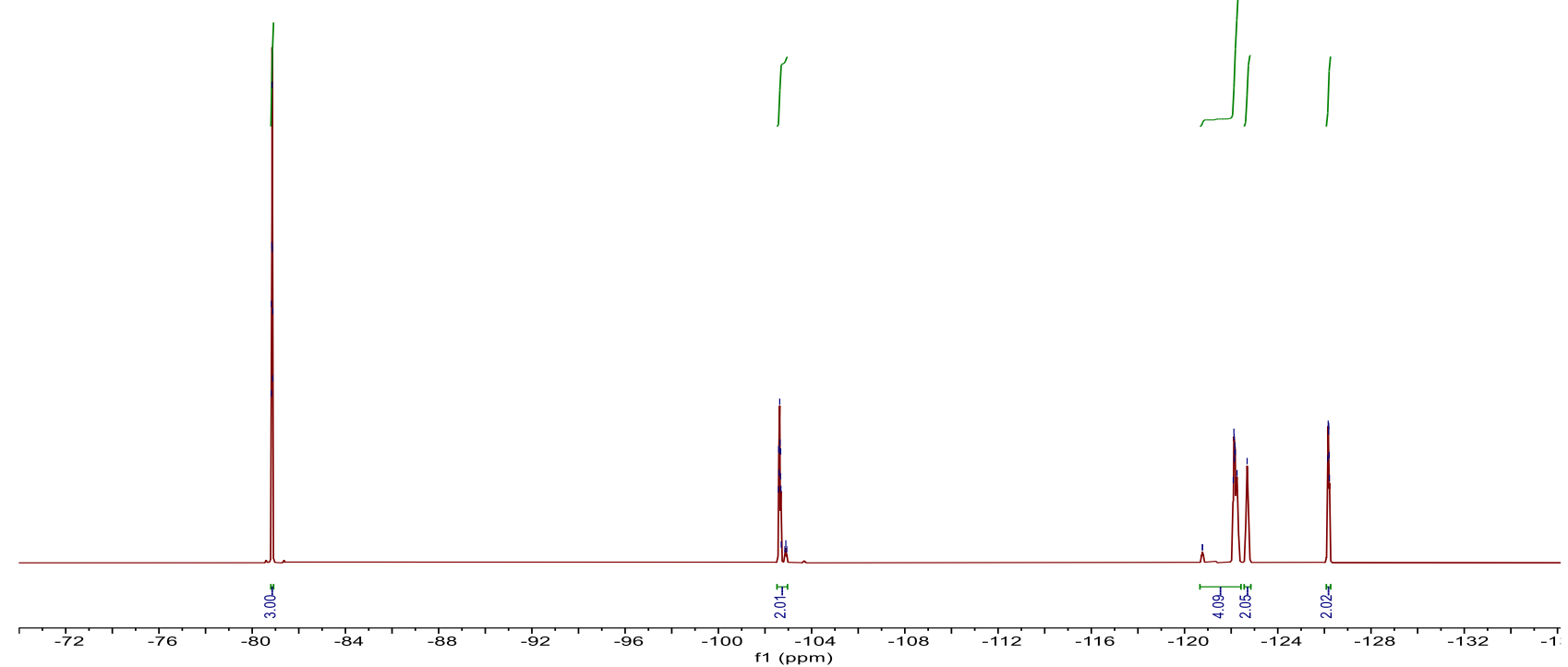

\section{1,3,5-Trimethoxy-2-(perfluorooctyl)benzene (14)}

${ }^{1} \mathbf{H}$ NMR: (400 MHz, $\left.\mathrm{CDCl}_{3}\right)$

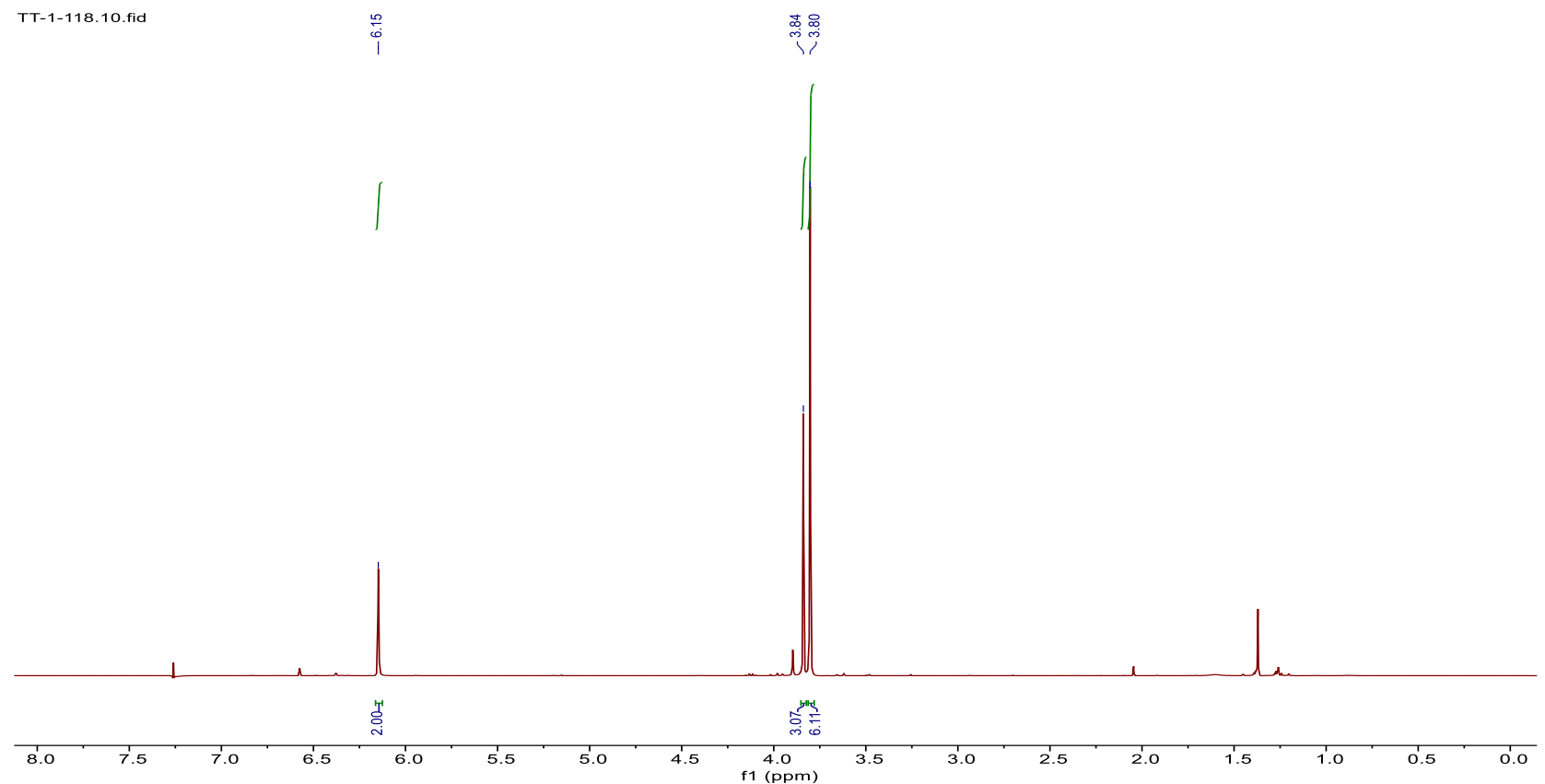


1,3,5-Trimethoxy-2-(perfluorooctyl)benzene (14)

${ }^{13}$ C NMR: (201 MHz, $\mathrm{CDCl}_{3}$ )

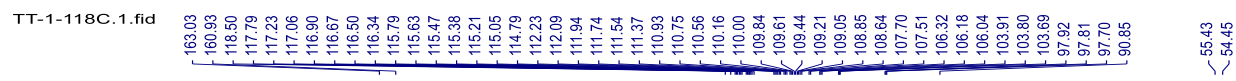

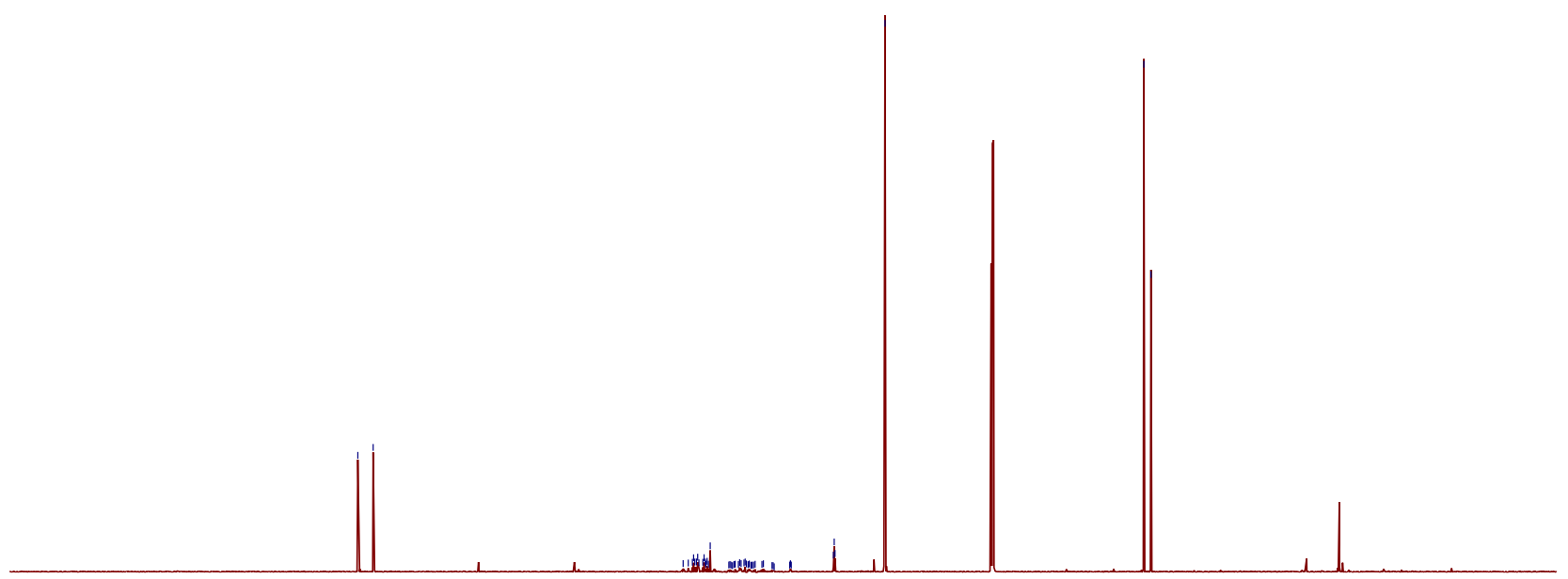

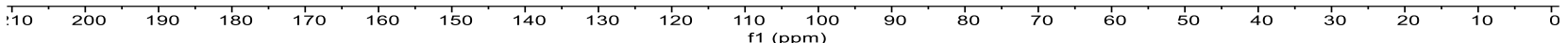

${ }^{19}$ F NMR: $\left(376 \mathrm{MHz}, \mathrm{CDCl}_{3}\right)$

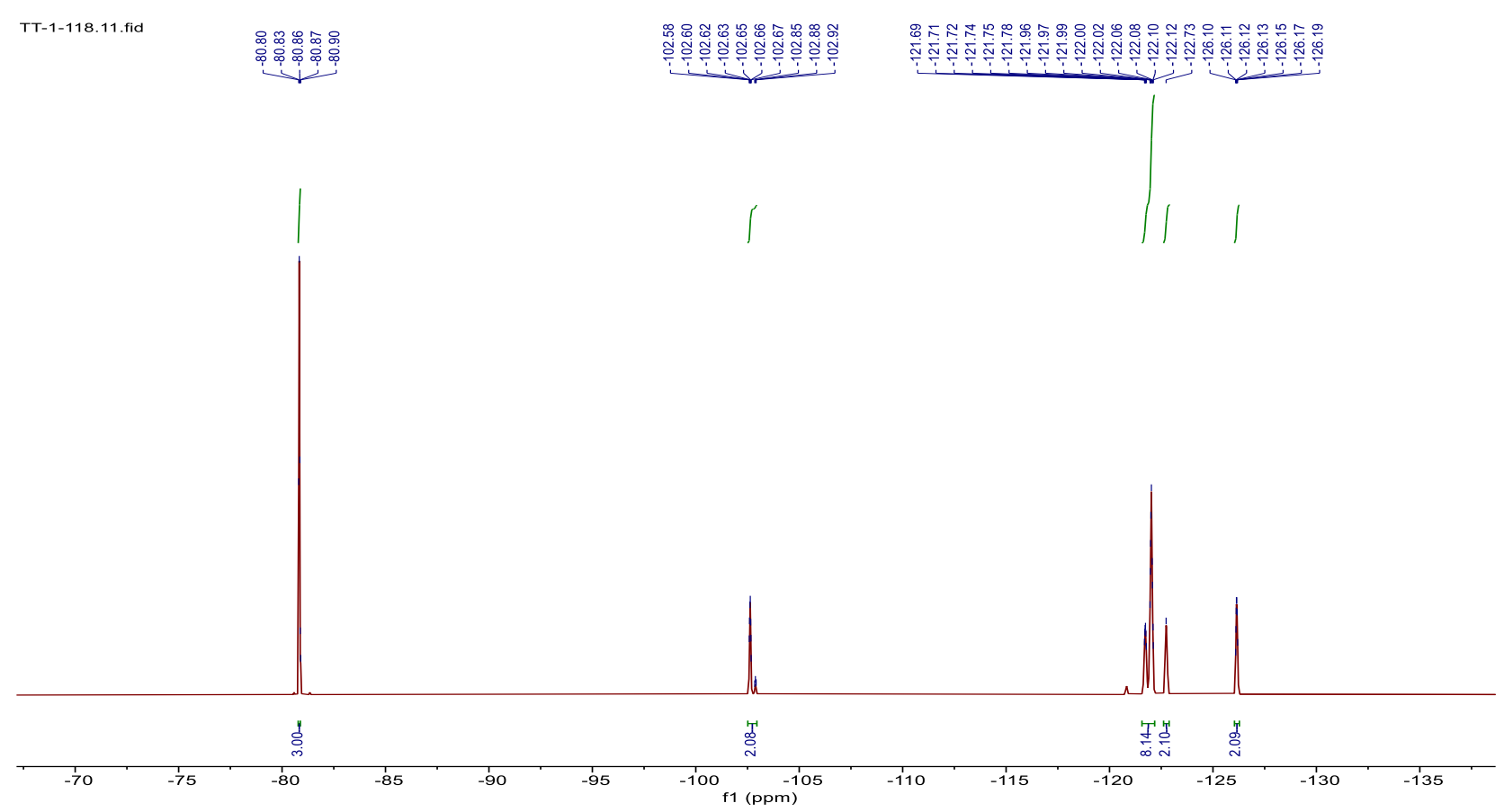




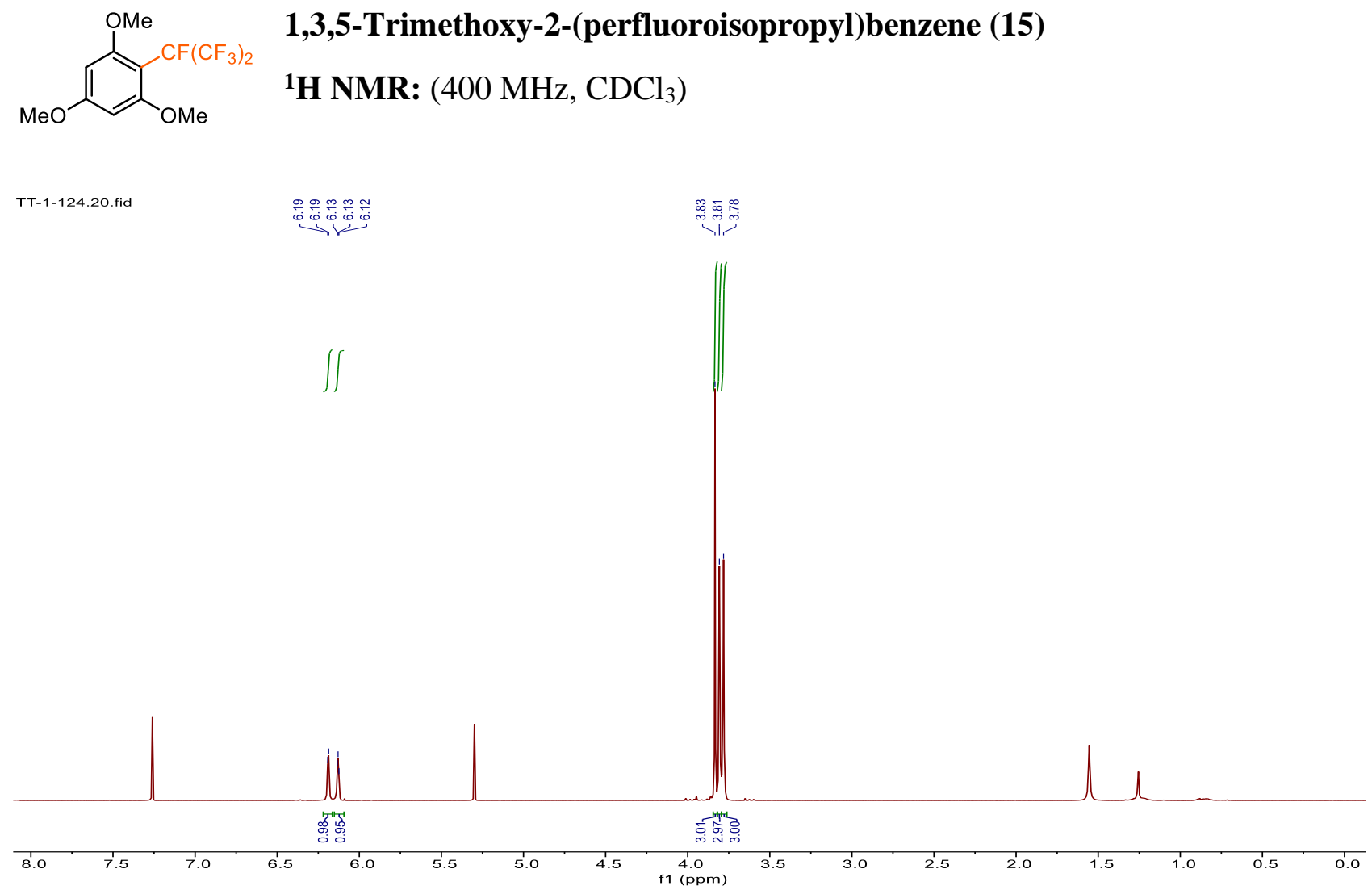

${ }^{13}$ C NMR: (201 MHz, $\mathrm{CDCl}_{3}$ )

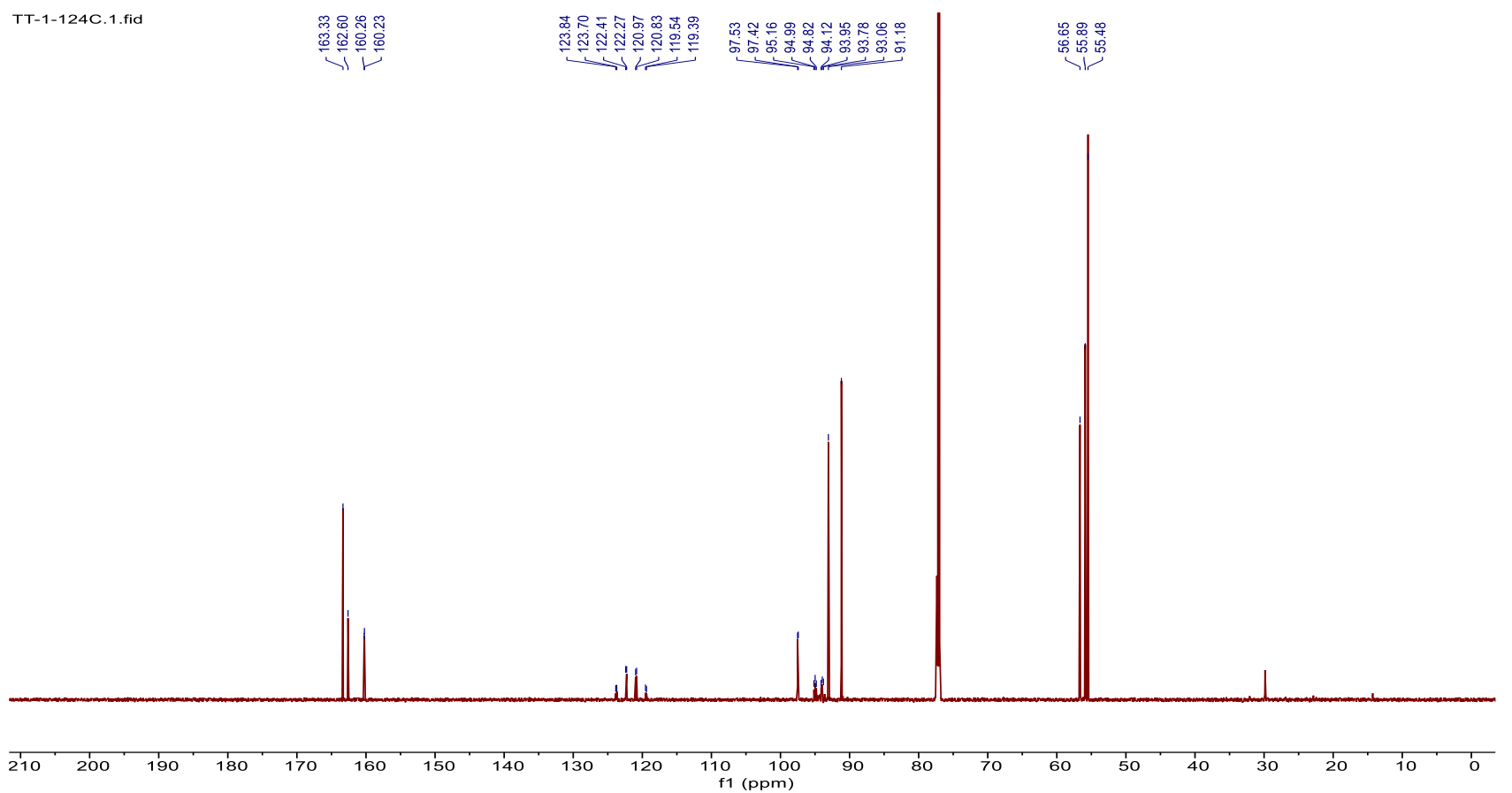


OMe

TT-1-124-F.10.fid 䙵离

1,3,5-Trimethoxy-2-(perfluoroisopropyl)benzene (15)

${ }^{19}$ F NMR: $\left(376 \mathrm{MHz}, \mathrm{CDCl}_{3}\right)$

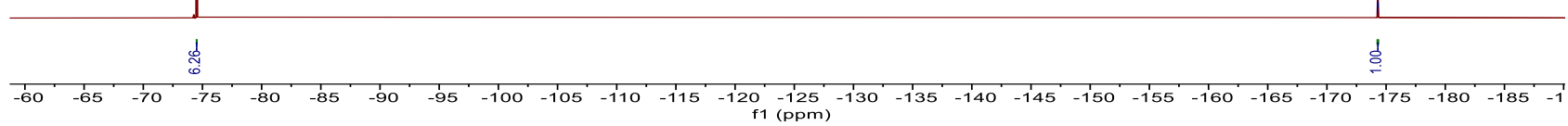
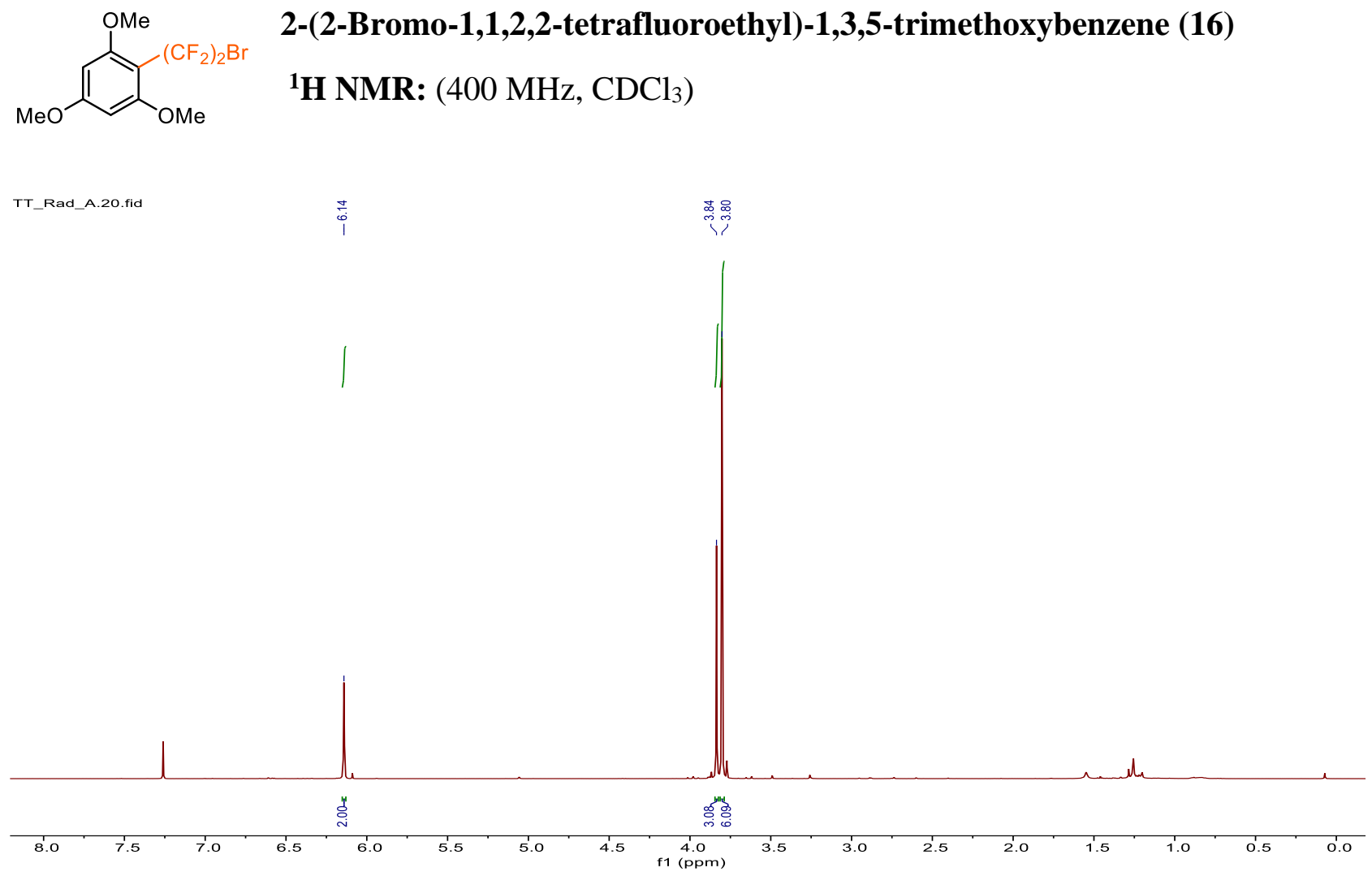

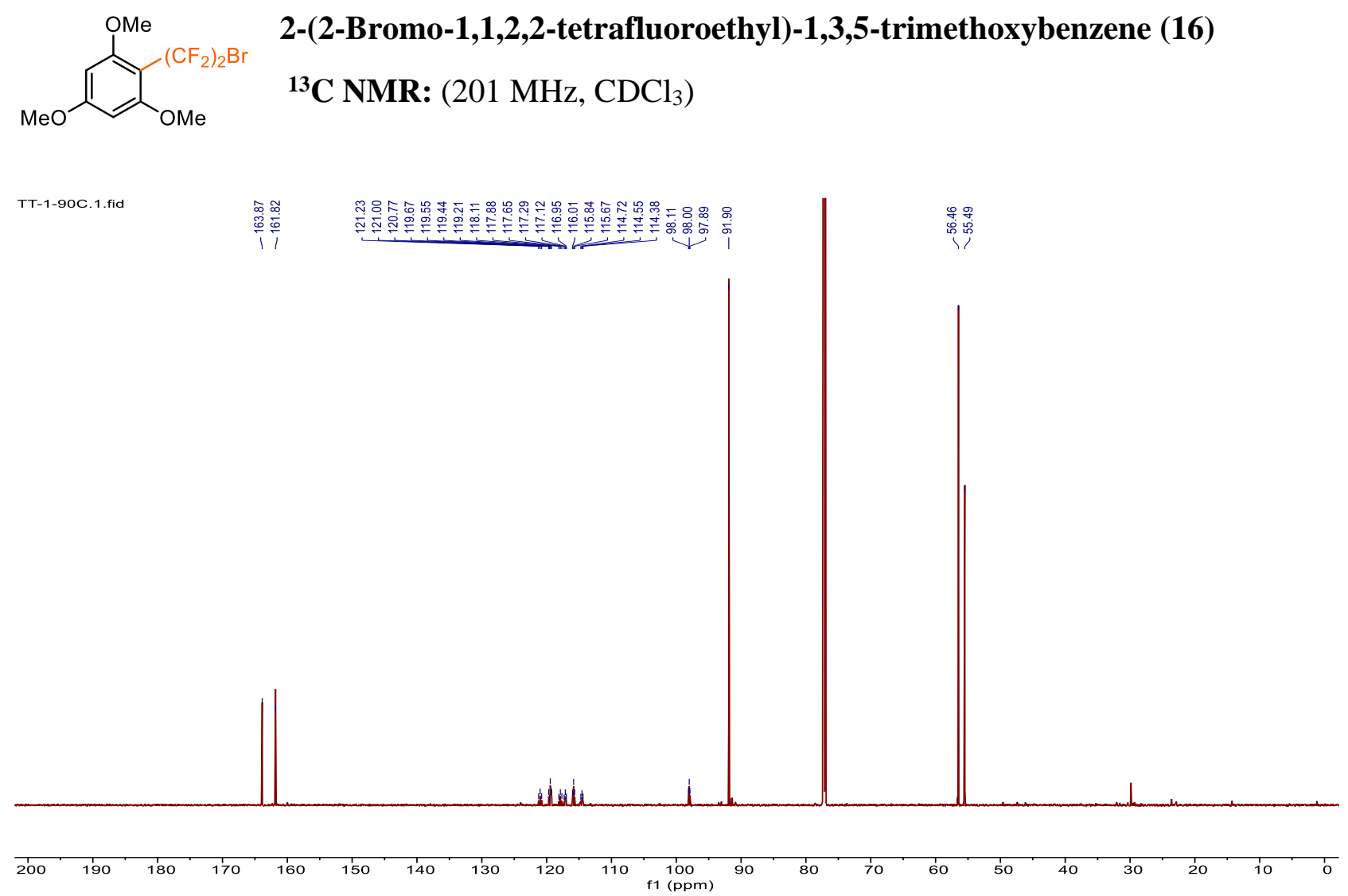

${ }^{19}$ F NMR: (376 MHz, $\left.\mathrm{CDCl}_{3}\right)$

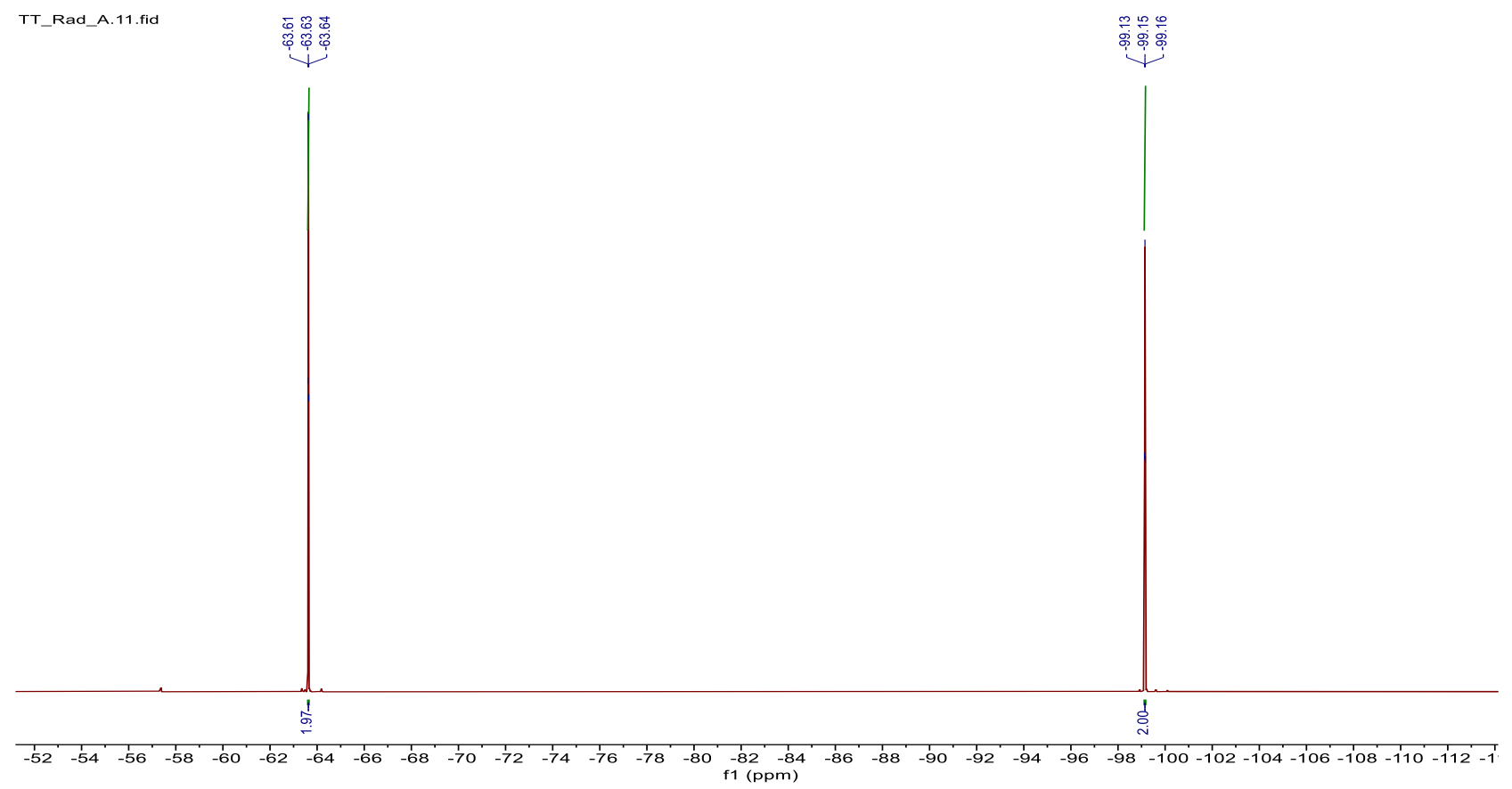




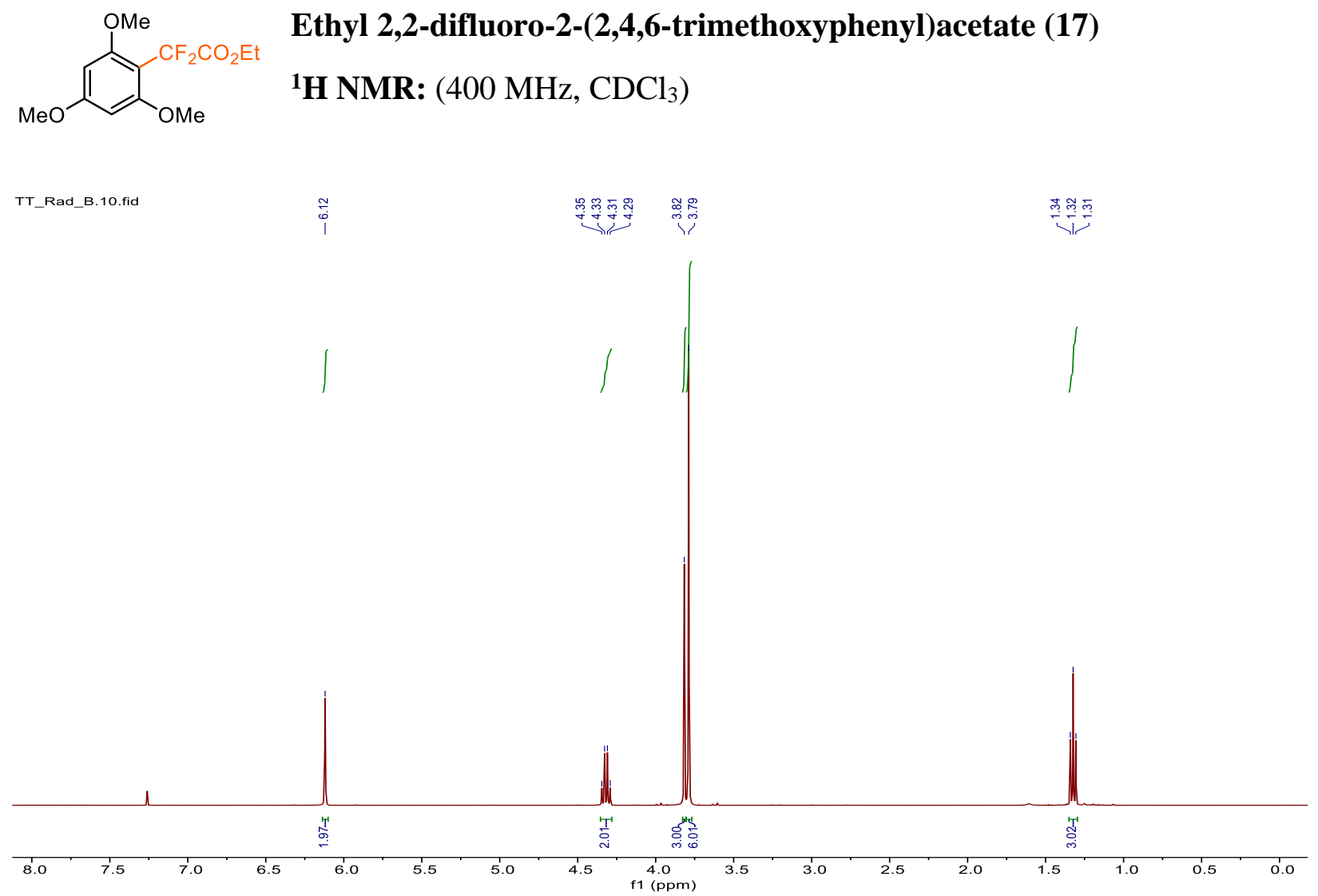

${ }^{13}$ C NMR: (376 MHz, $\left.\mathrm{CDCl}_{3}\right)$

\begin{tabular}{|c|c|}
\hline TT-1-91C.1.fid & 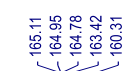 \\
\hline
\end{tabular}

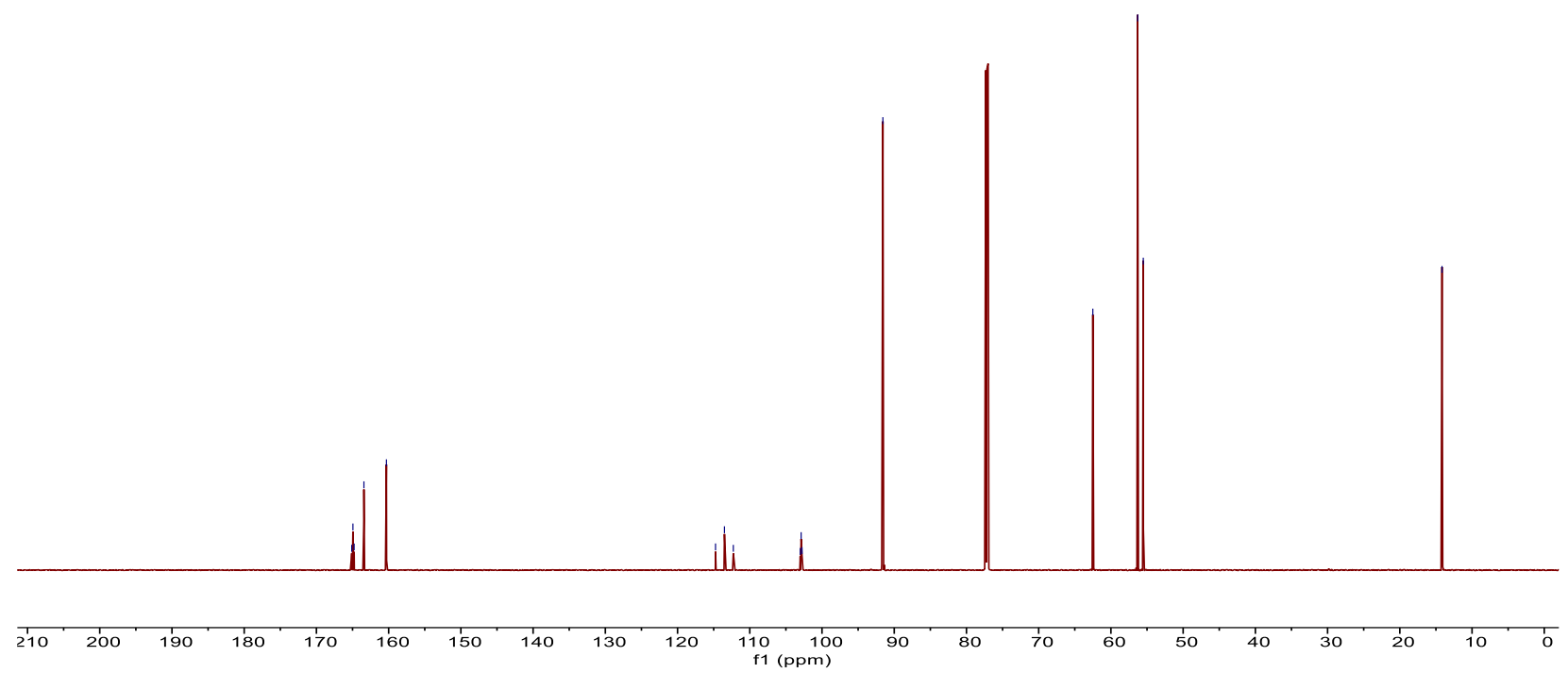


<smiles>CCOC(=O)C(F)(F)c1c(OC)cc(OC)cc1OC</smiles>

TT_Rad_B.11.fid
Ethyl 2,2-difluoro-2-(2,4,6-trimethoxyphenyl)acetate (17)

${ }^{19}$ F NMR: (376 MHz, $\left.\mathrm{CDCl}_{3}\right)$

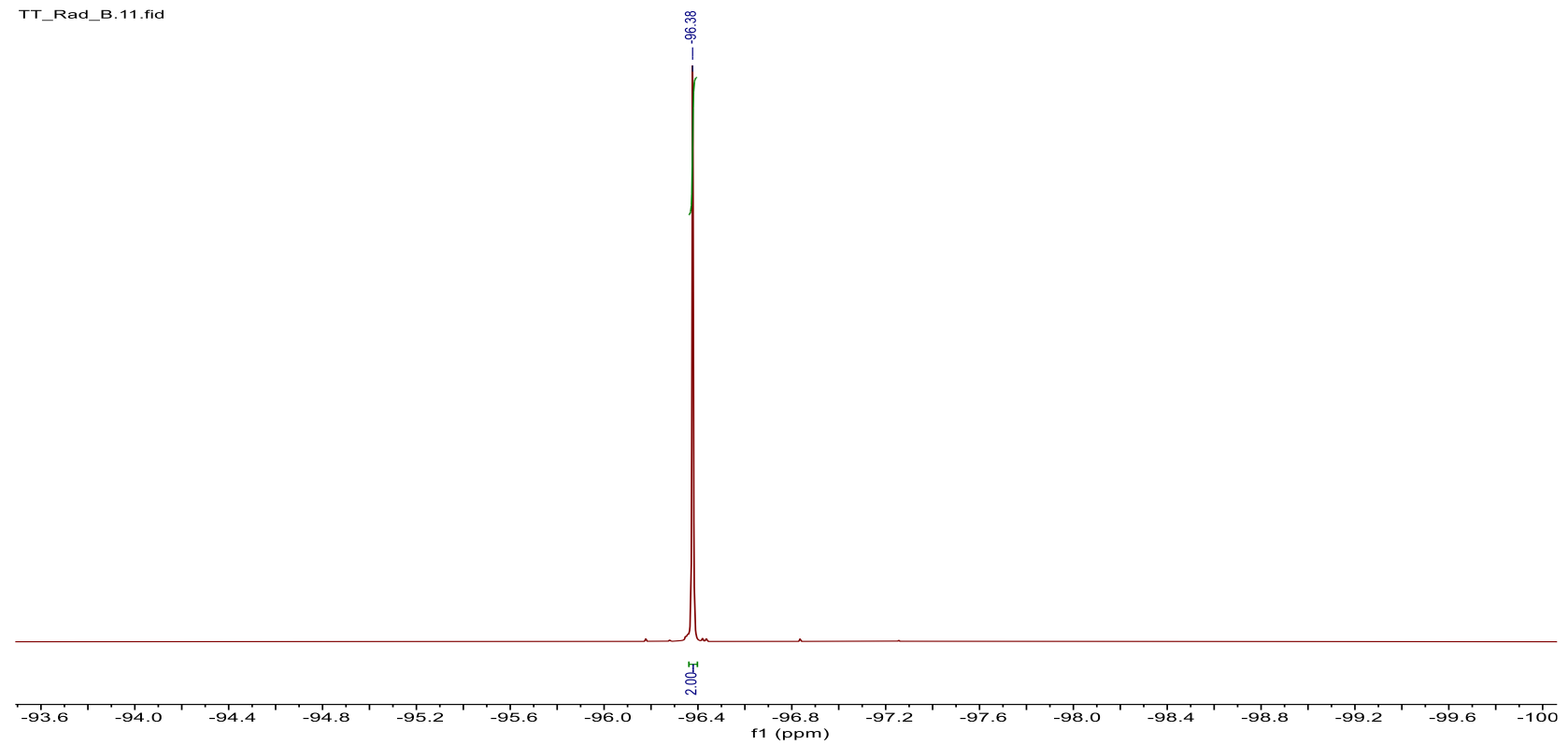

$\curvearrowright\left(\mathrm{CF}_{2}\right)_{3} \mathrm{CF}_{3}$

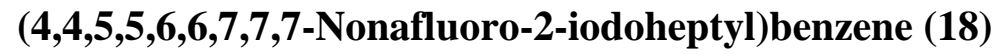
${ }^{1}$ H NMR: (400 MHz, $\left.\mathrm{CDCl}_{3}\right)$

Note: Compound is extremely hygroscopic. $\mathrm{H}_{2} \mathrm{O}$ could not be fully removed even after prolonged drying under high vacuum. CR-1-63.10.fid -

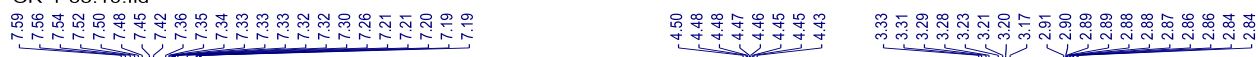
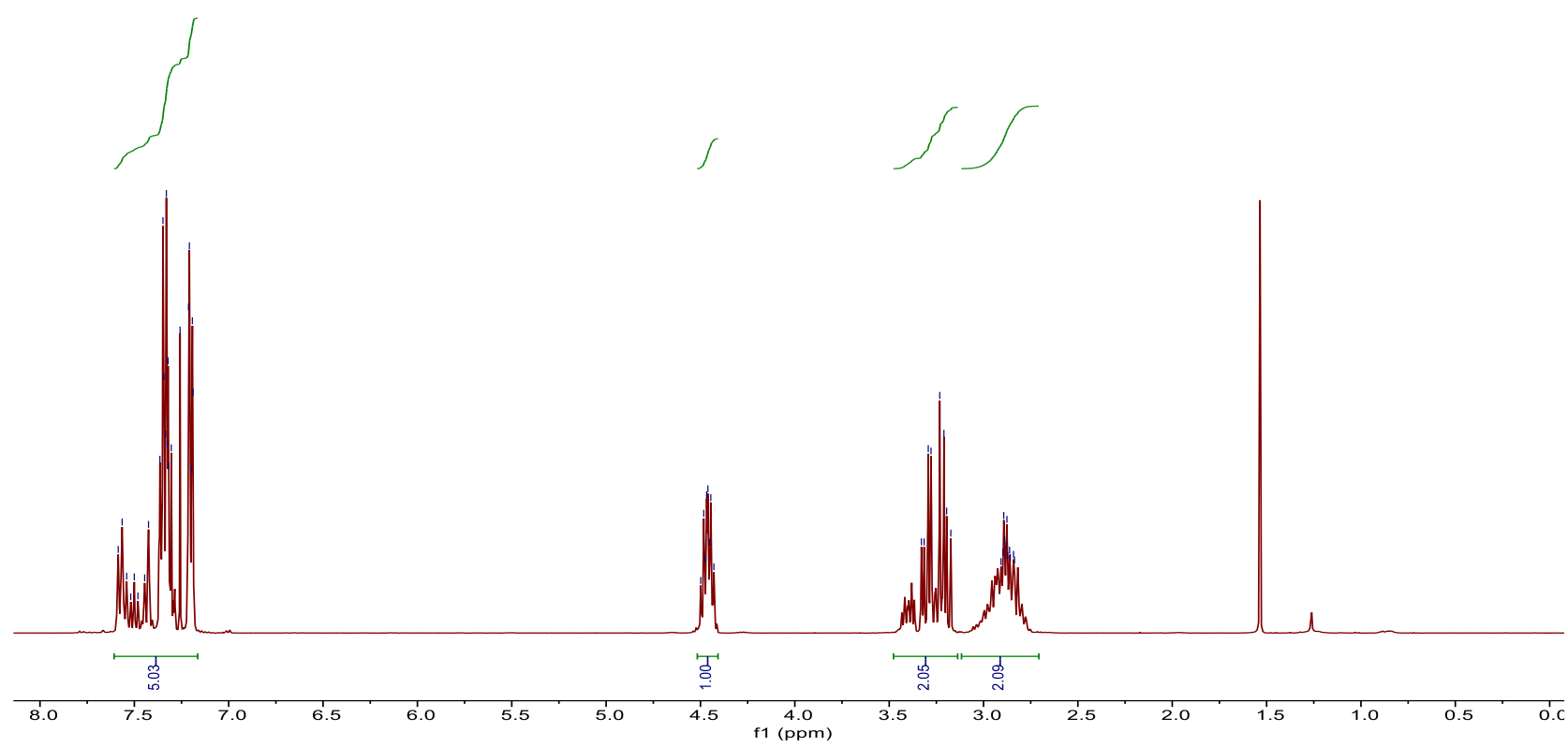
$\left.\mathrm{CPF}_{2}\right)_{3} \mathrm{CF}_{3}$

(4,4,5,5,6,6,7,7,7-Nonafluoro-2-iodoheptyl)benzene (18)

${ }^{19}$ C NMR: (201 MHz, $\left.\mathrm{CDCl}_{3}\right)$

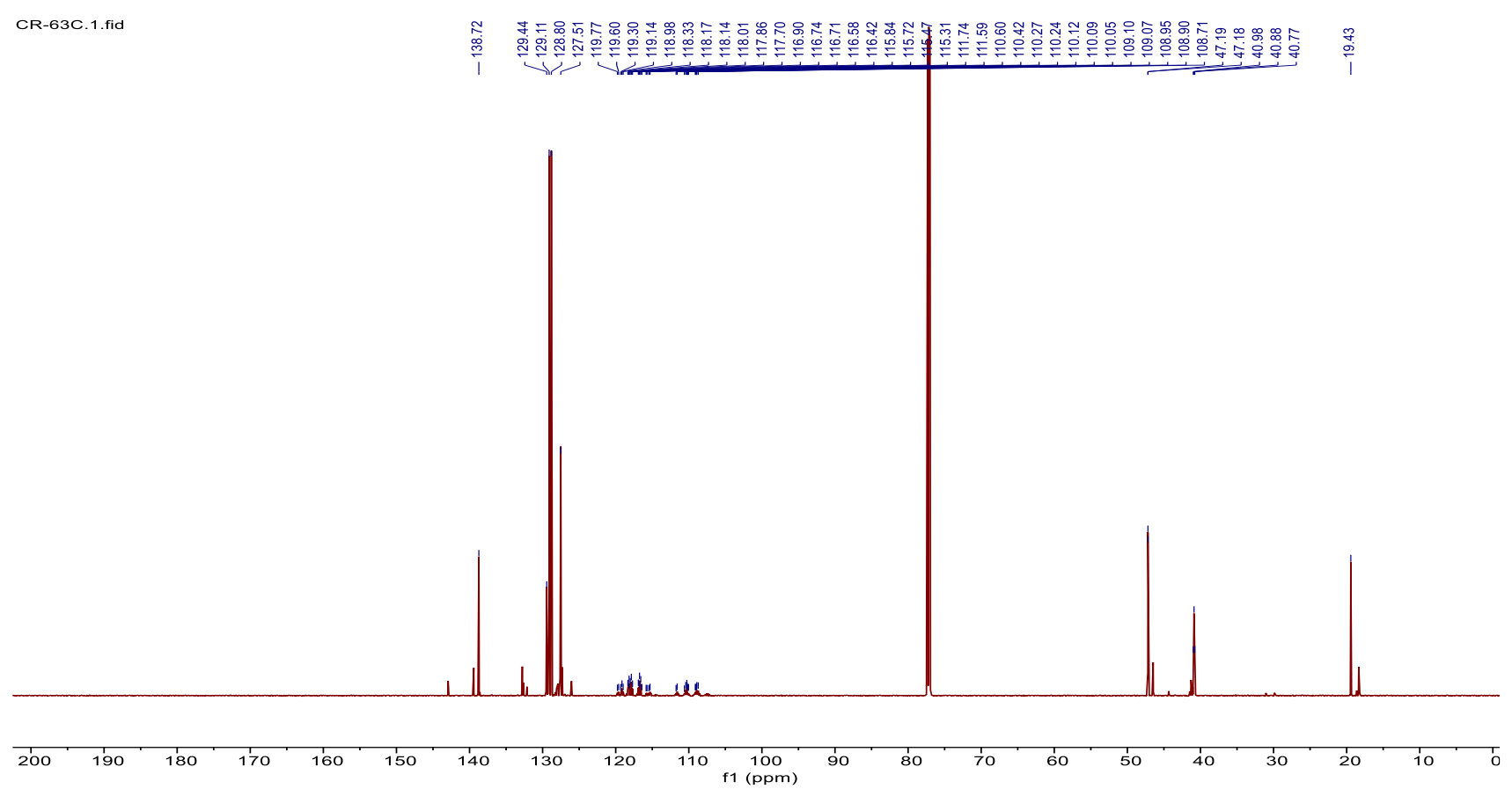

${ }^{19}$ F NMR: $\left(376 \mathrm{MHz}, \mathrm{CDCl}_{3}\right)$

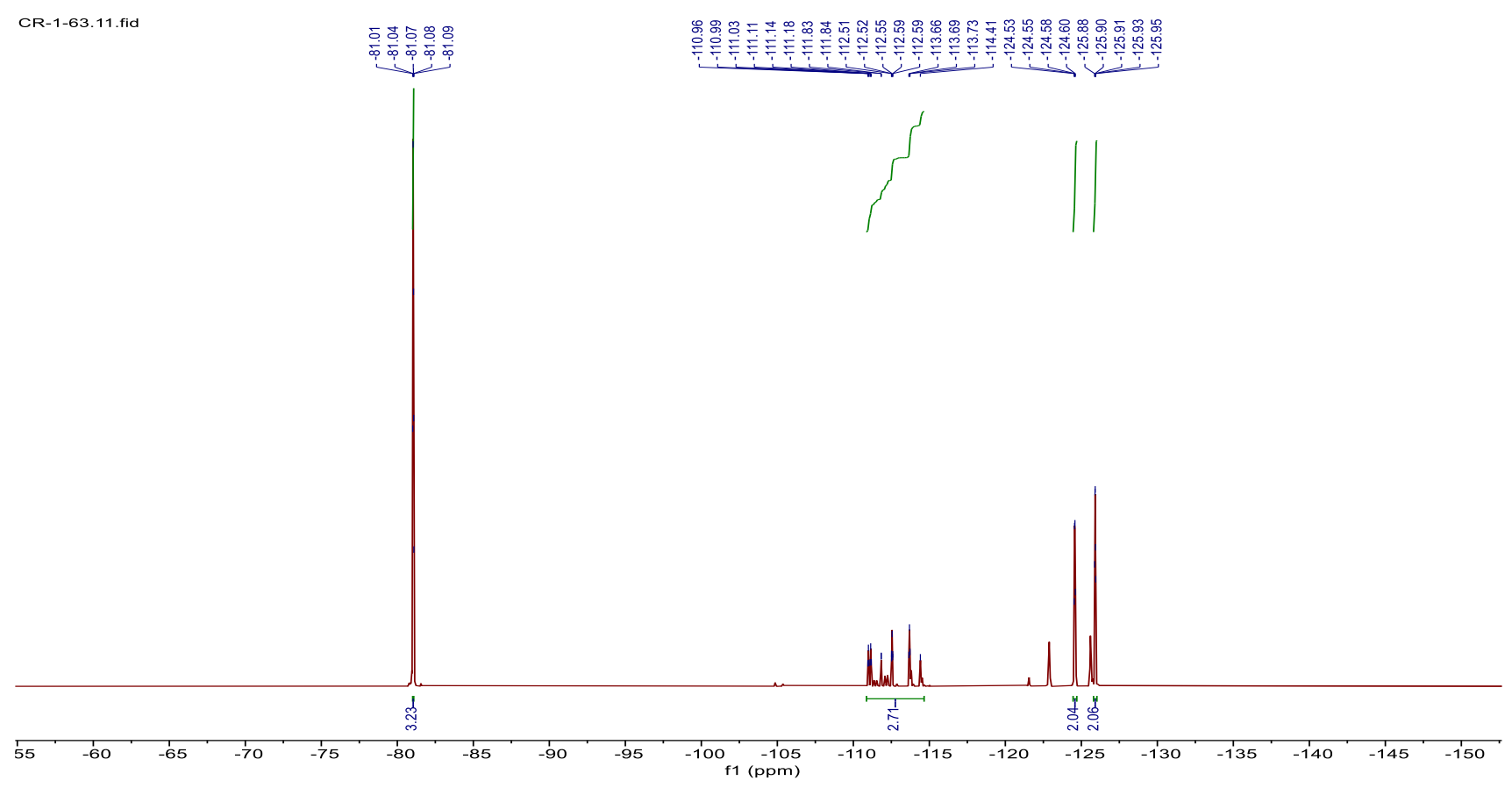




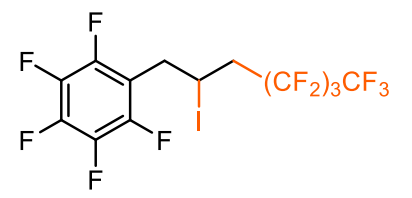

\section{1,2,3,4,5-pentafluoro-6-(4,4,5,5,6,6,7,7,7-nonafluoro-2-iodoheptyl)- benzene (19) \\ ${ }^{1}$ H NMR: $\left(400 \mathrm{MHz}, \mathrm{CDCl}_{3}\right)$}

Note: Compound is extremely hygroscopic. $\mathrm{H}_{2} \mathrm{O}$ could not be fully removed even after prolonged drying under high vacuum.

CR-1-65.10.fid

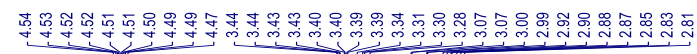
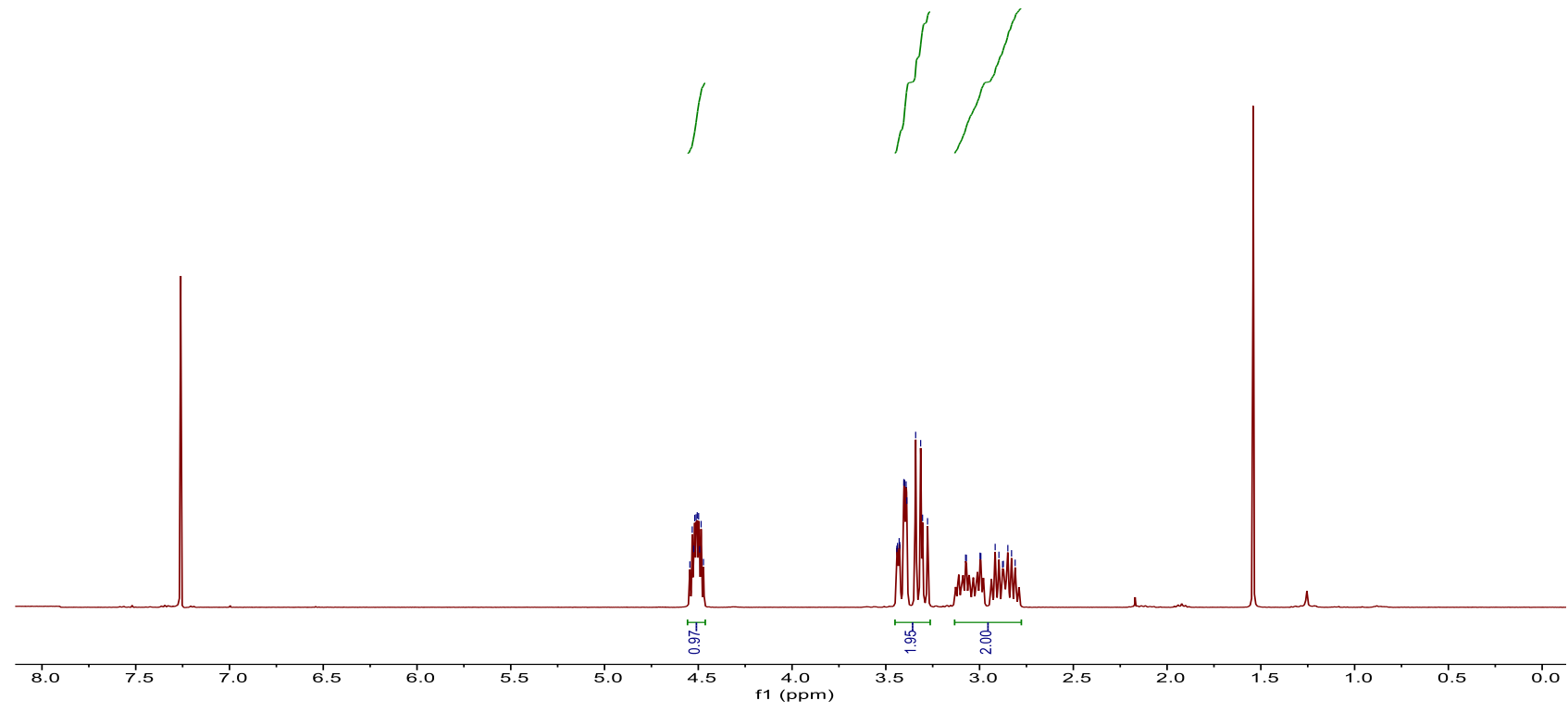

${ }^{19}$ C NMR: (201 MHz, $\left.\mathrm{CDCl}_{3}\right)$

CR-65-C.1.fid -

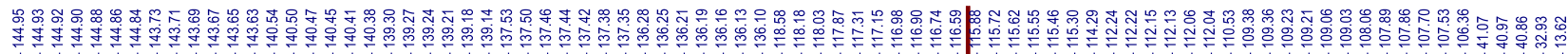

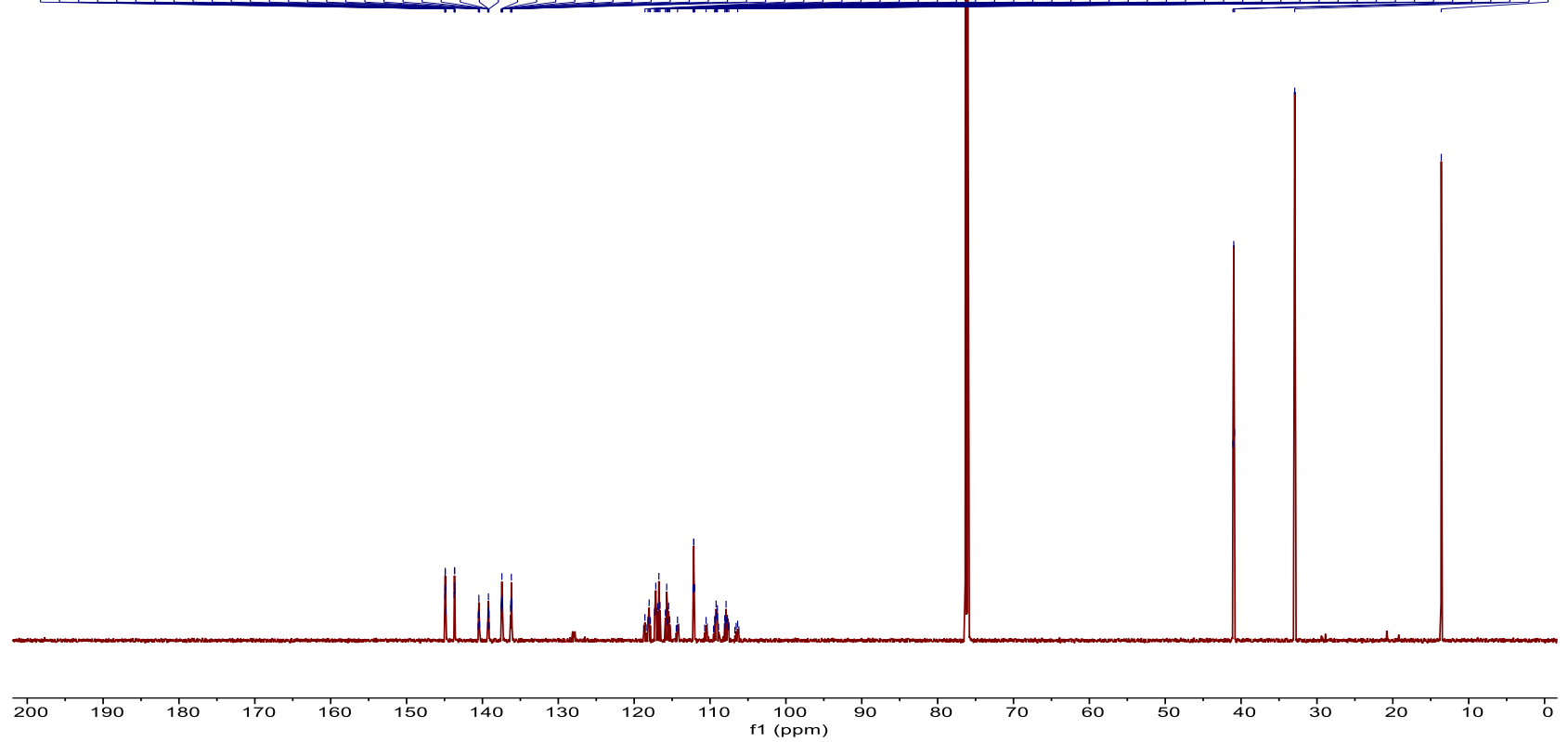



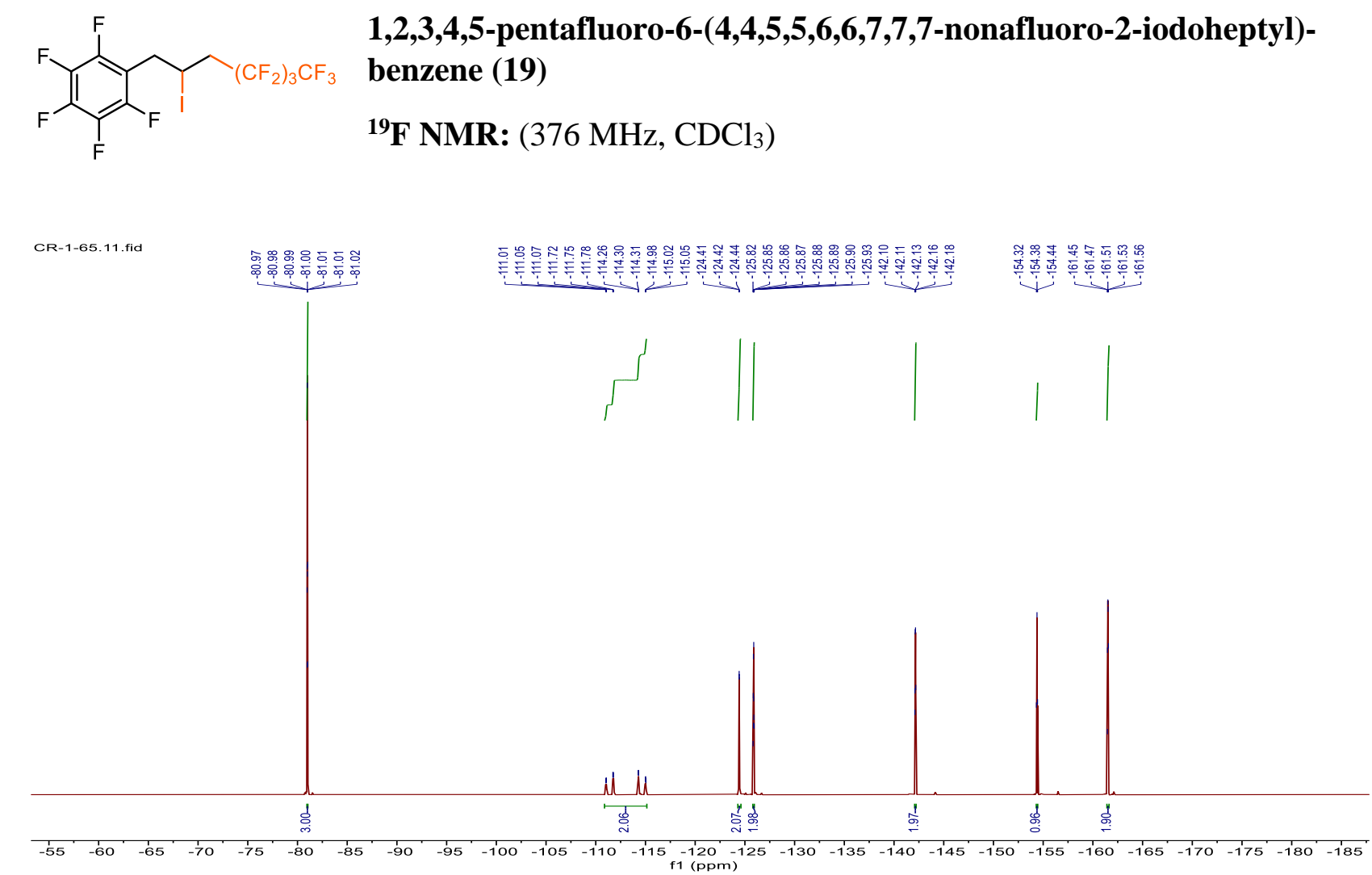

\section{1,2,3,4,5-pentafluoro-6-(4,4,5,5,6,6,7,7,7-nonafluoro-2-iodoheptyl)-} benzene (19)

${ }^{19}$ F NMR: $\left(376 \mathrm{MHz}, \mathrm{CDCl}_{3}\right)$

CR-1-64.10.fid ${ }^{1}$ H NMR: $\left(400 \mathrm{MHz}, \mathrm{CDCl}_{3}\right)$
BocHN $\Upsilon_{\left(\mathrm{CF}_{2}\right)_{3} \mathrm{CF}_{3}}$ Tert-butyl (4,4,5,5,6,6,7,7,7-nonafluoro-2-iodoheptyl)carbamate (20)

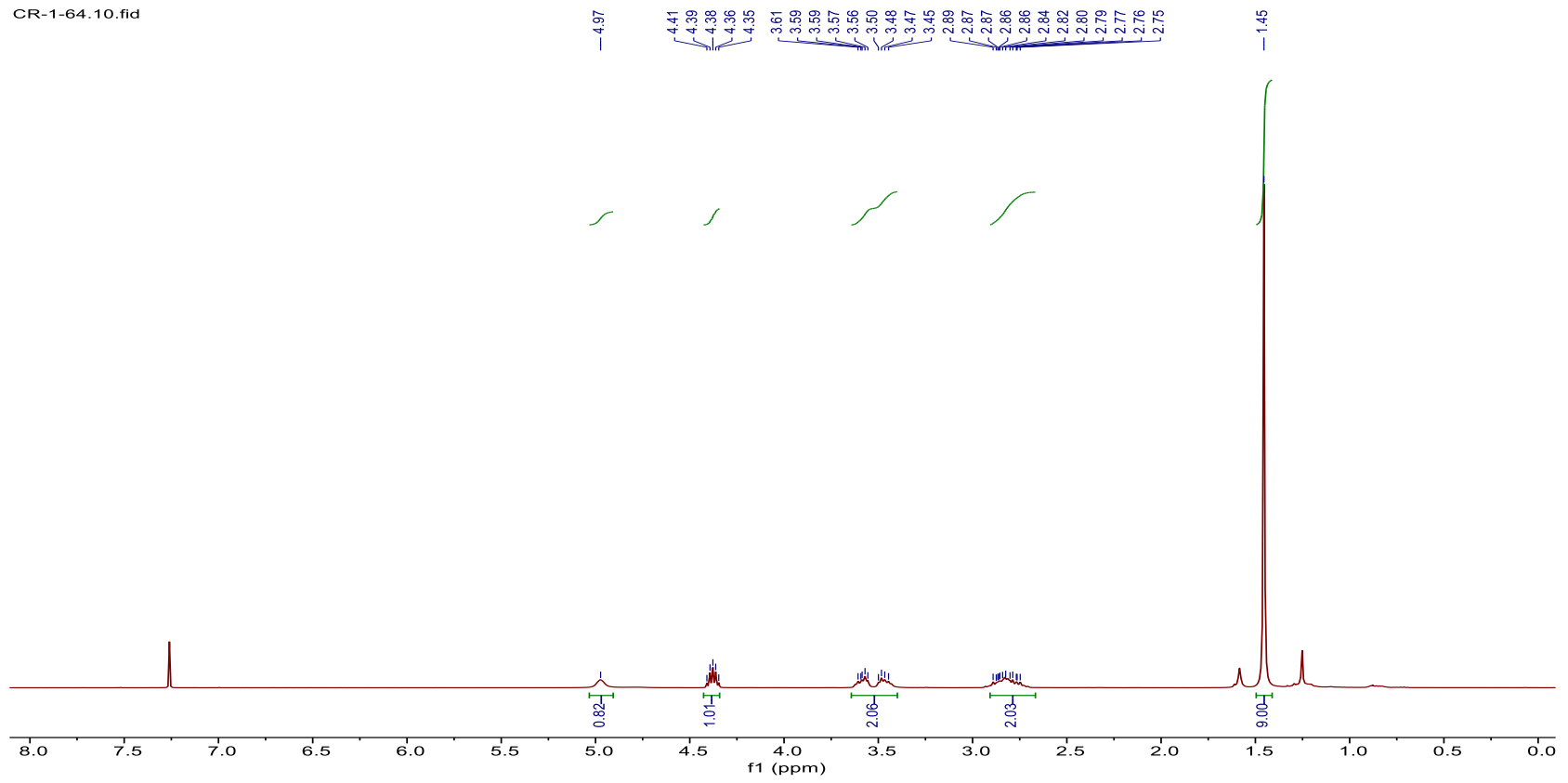


$\mathrm{BocHN} \bigcirc\left(\mathrm{CF}_{2}\right)_{3} \mathrm{CF}_{3}$

Tert-butyl (4,4,5,5,6,6,7,7,7-nonafluoro-2-iodoheptyl)carbamate (20)

${ }^{13}$ C NMR: (101 MHz, $\left.\mathrm{CDCl}_{3}\right)$

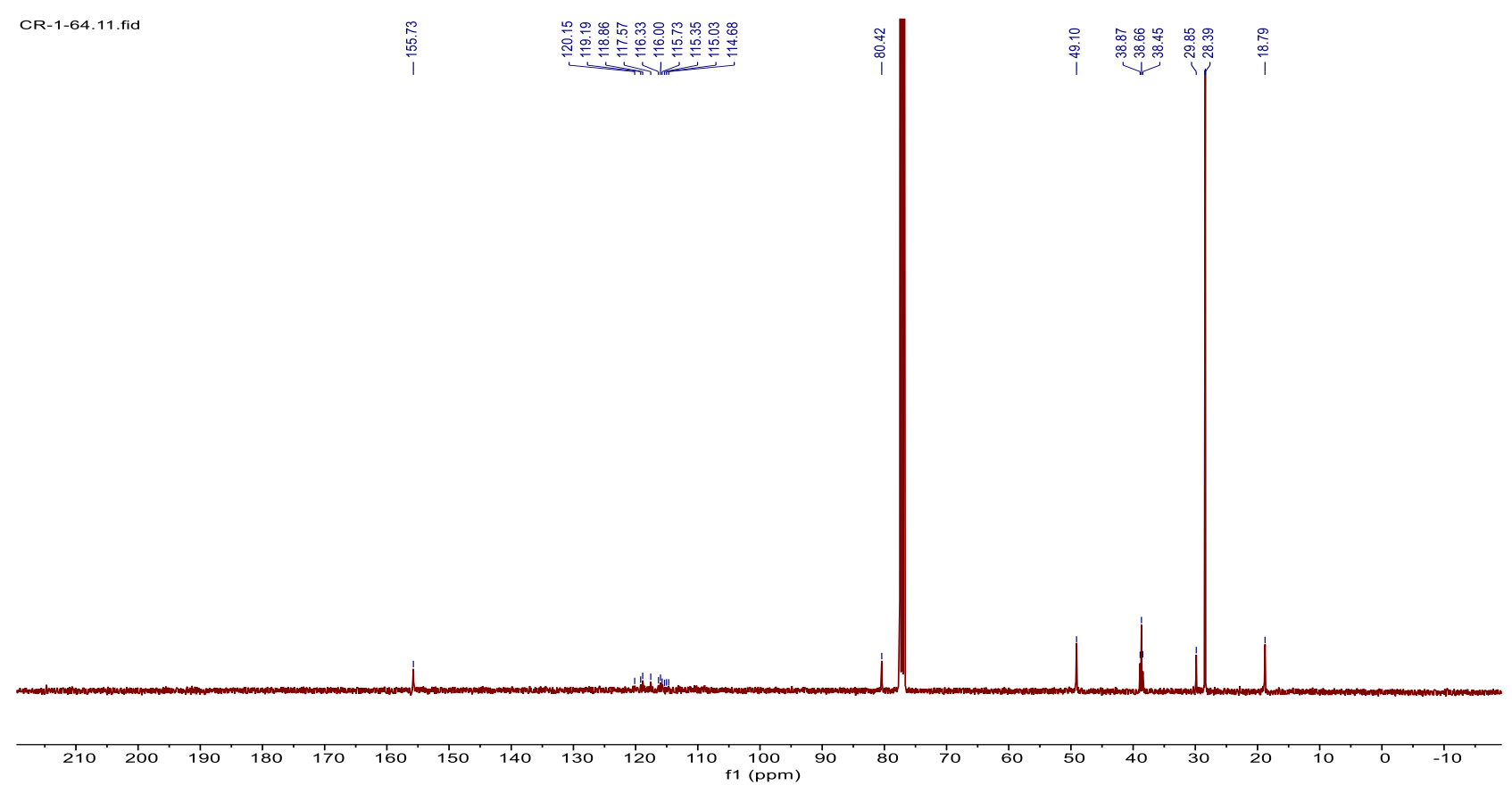

${ }^{19}$ F NMR: $\left(376 \mathrm{MHz}, \mathrm{CDCl}_{3}\right)$

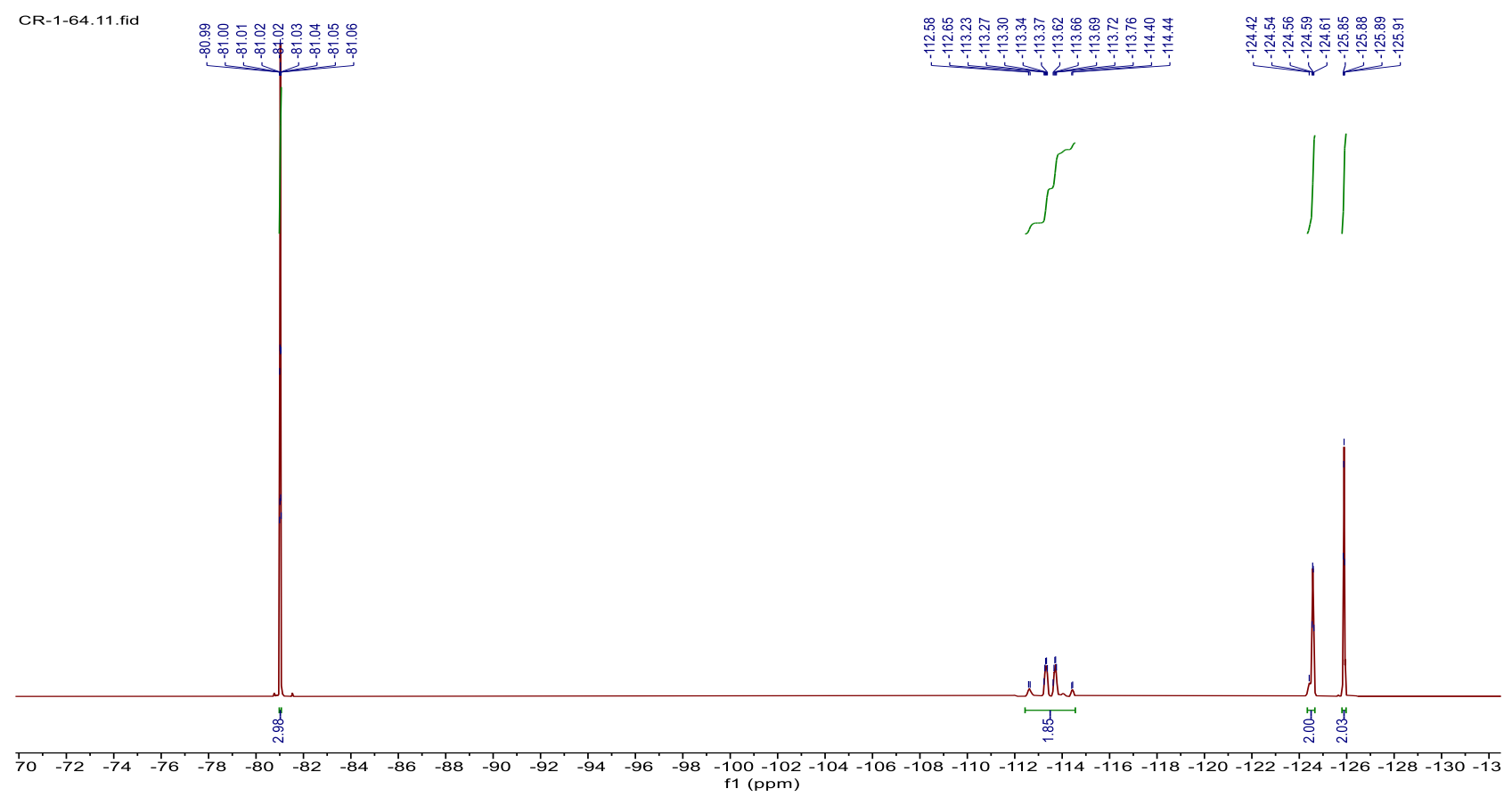




\section{$\overbrace{0}^{\circ} \widehat{\sim}\left(\mathrm{CF}_{2}\right)_{3} \mathrm{CF}_{3} \quad$ 2-(((4,4,5,5,6,6,7,7,7-nonafluoro-2-iodoheptyl)oxy)methyl)oxirane (21) ${ }^{1} \mathbf{H}$ NMR: $\left(400 \mathrm{MHz}, \mathrm{CDCl}_{3}\right)$}

Note: Compound is extremely hygroscopic. $\mathrm{H}_{2} \mathrm{O}$ could not be fully removed even after prolonged drying under high vacuum.

TT-1-130_F1.10.fid

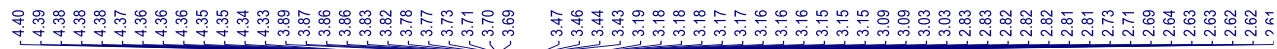
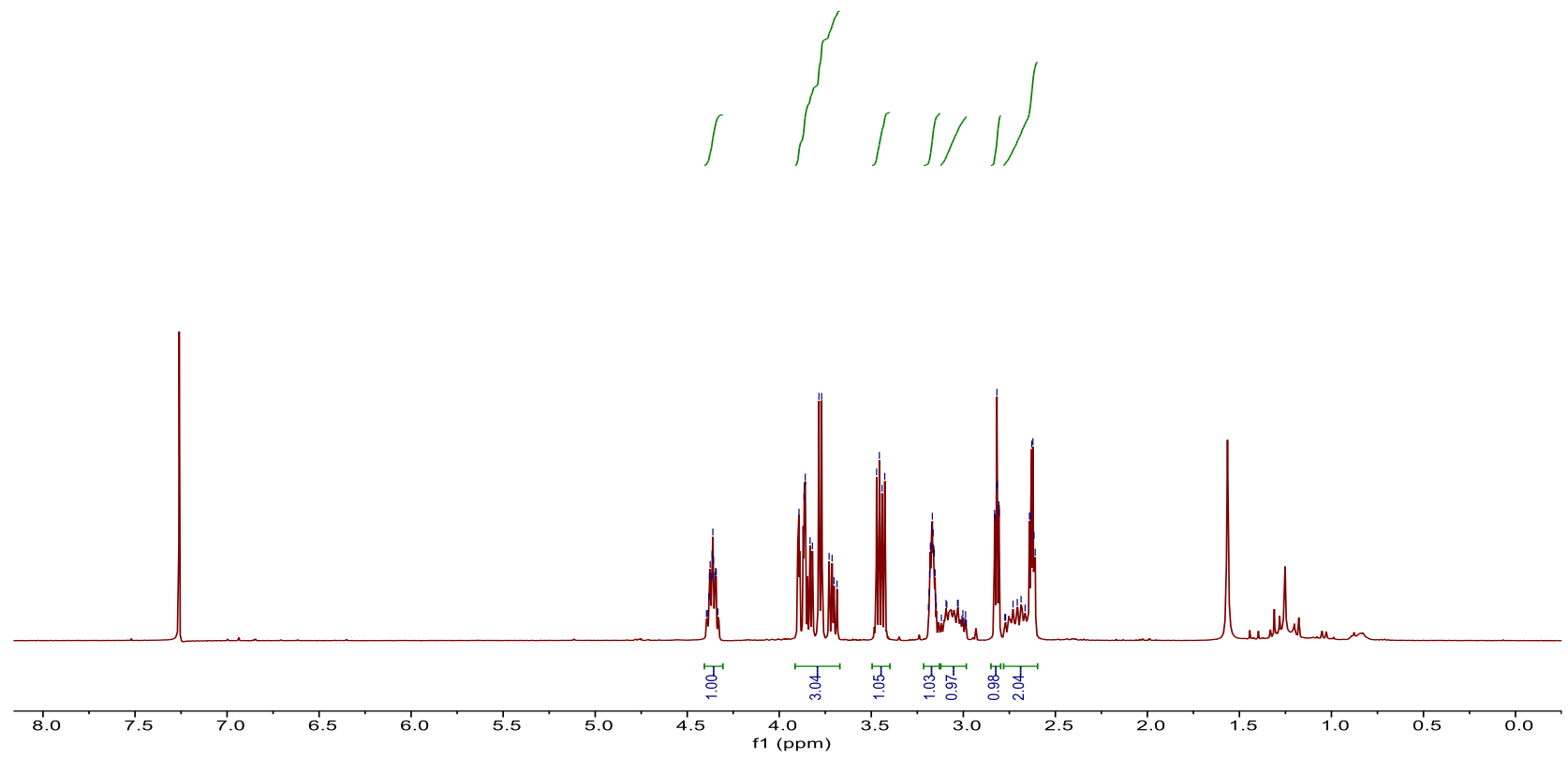

${ }^{13}$ C NMR: (201 MHz, $\left.\mathrm{CDCl}_{3}\right)$

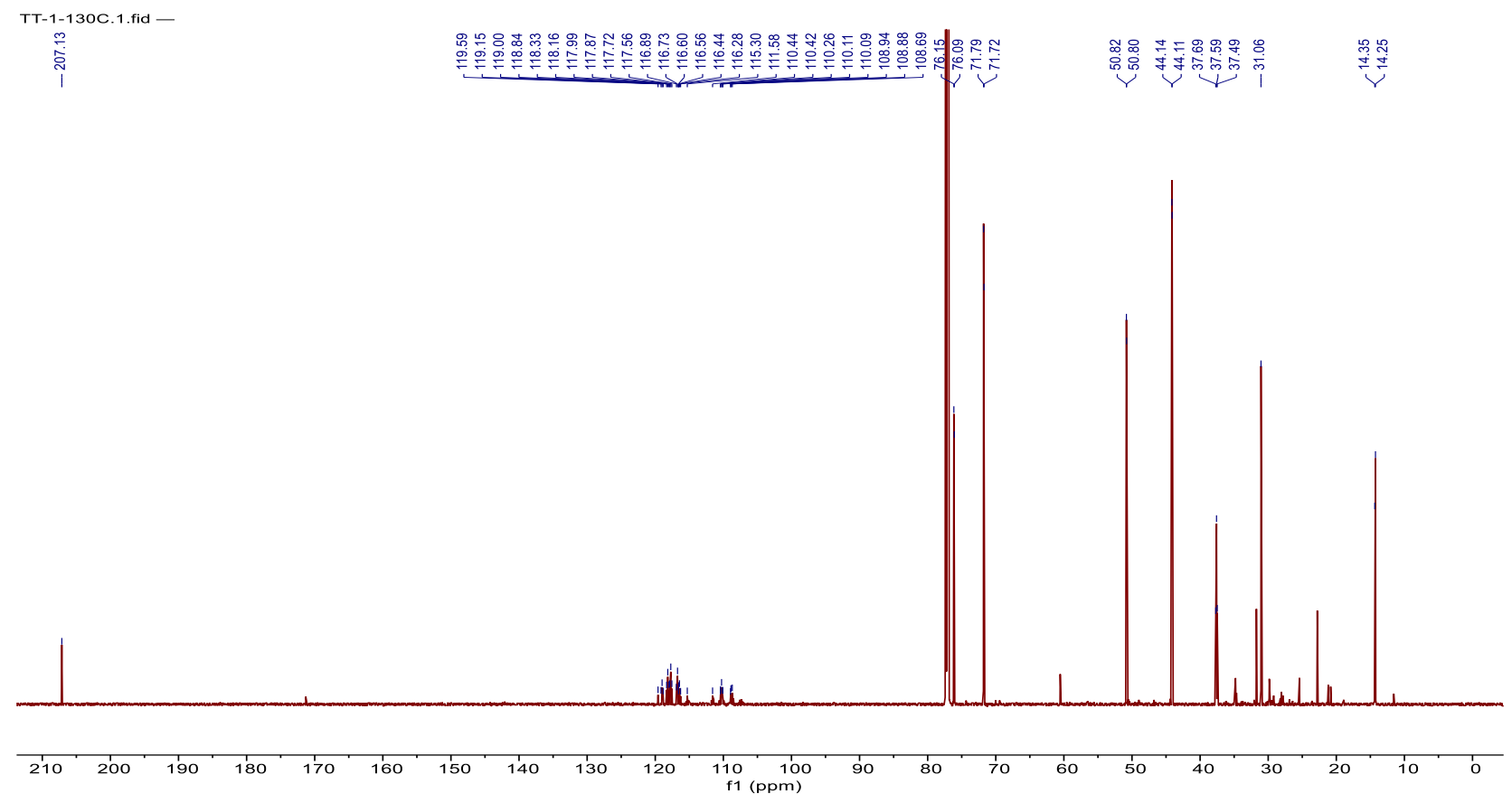




\section{$\overbrace{\circ}^{\circ} \Upsilon \mathrm{CF}_{2})_{3} \mathrm{CF}_{3} \quad$ 2-(((4,4,5,5,6,6,7,7,7-nonafluoro-2-iodoheptyl)oxy)methyl)oxirane (21) \\ ${ }^{19}$ F NMR: $\left(376 \mathrm{MHz}, \mathrm{CDCl}_{3}\right)$}

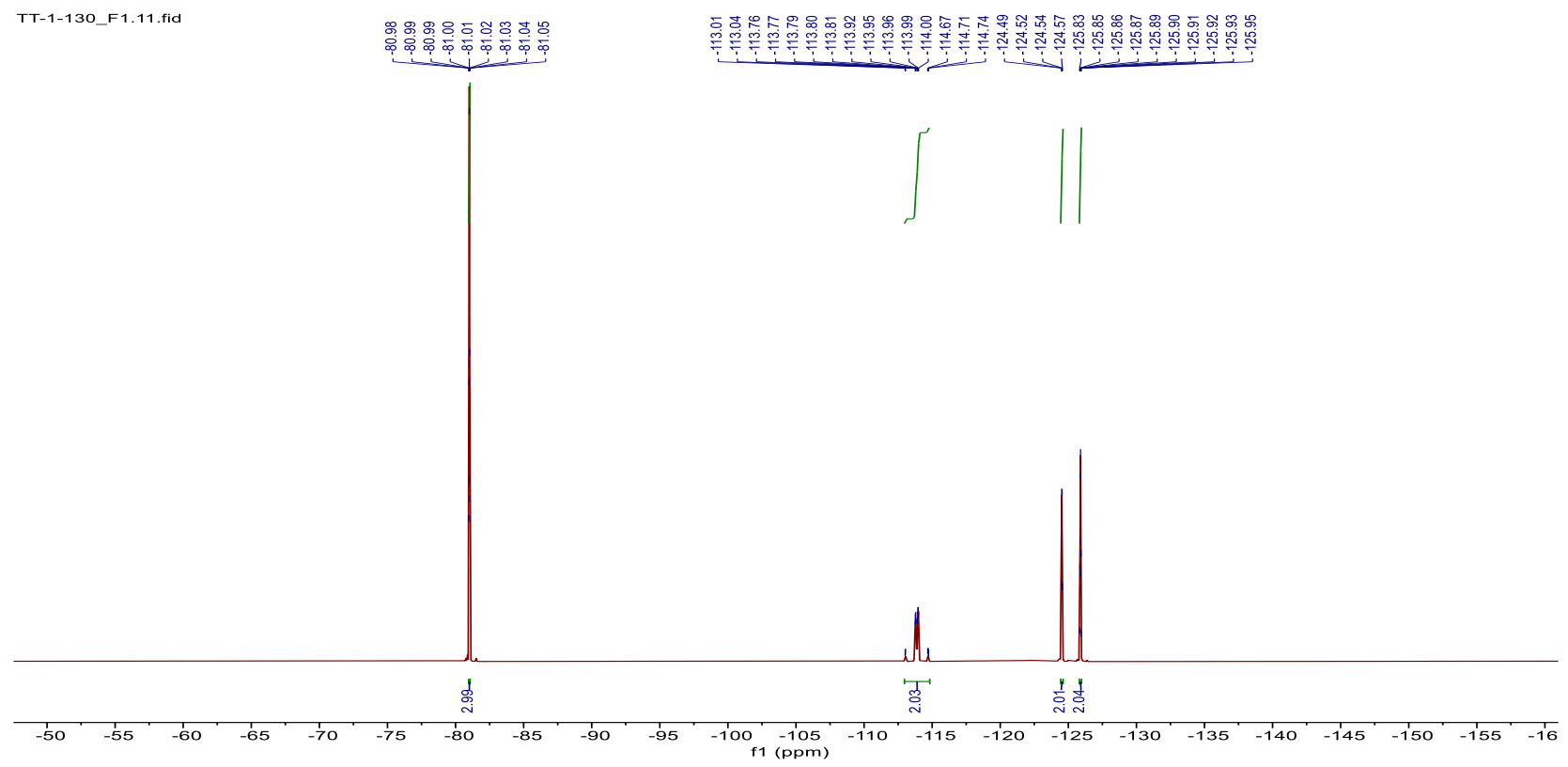

\section{1-Iodo-2-(perfluorobutyl)cyclooctane (22) (1.6:1 dr)}

${ }^{1}$ H NMR: (400 MHz, $\left.\mathrm{CDCl}_{3}\right)$

TT-1-126.10.fid

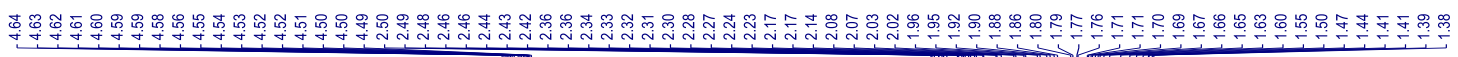

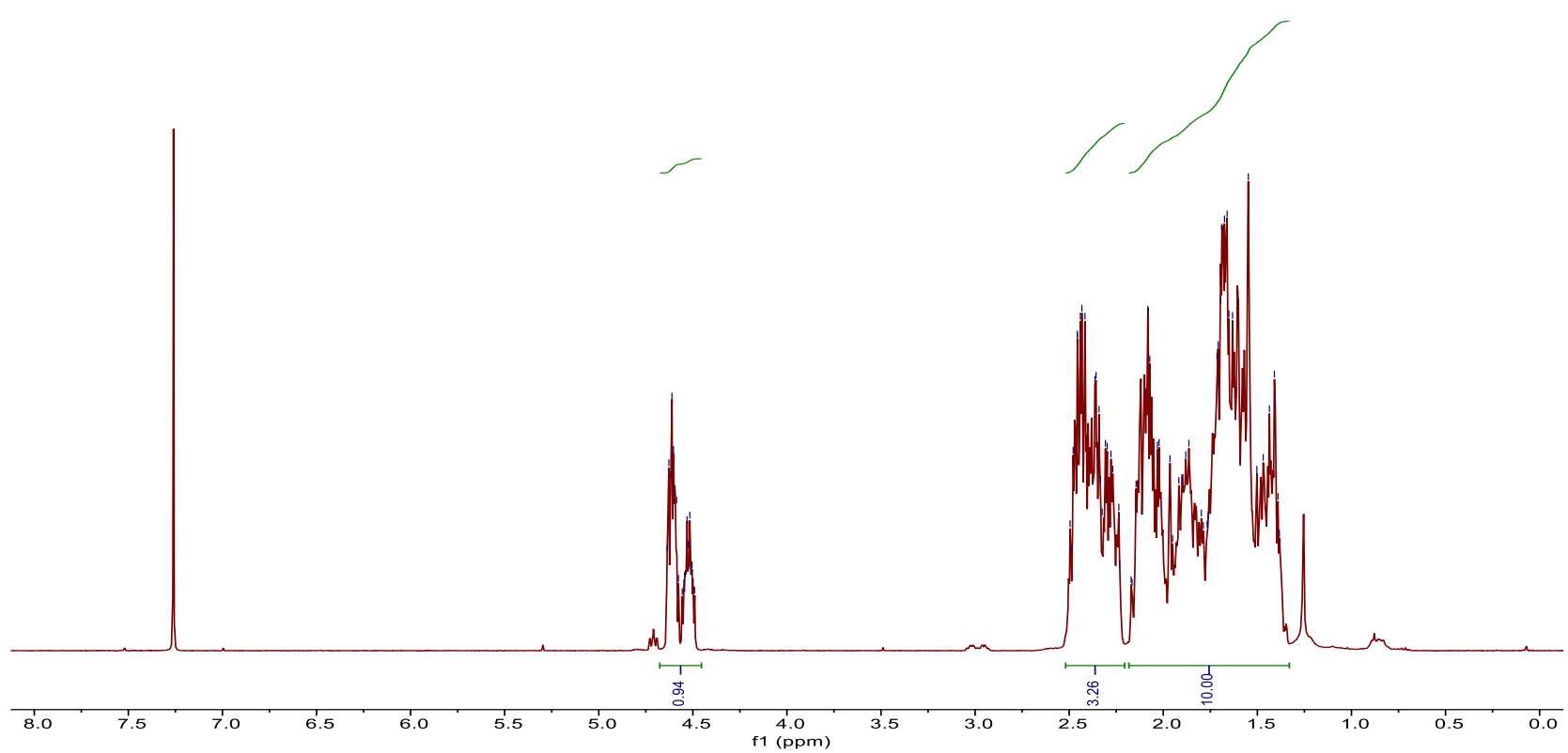




\section{1-Iodo-2-(perfluorobutyl)cyclooctane (22) (1.6:1 dr)}

${ }^{13}$ C NMR: (201 MHz, $\left.\mathrm{CDCl}_{3}\right)$

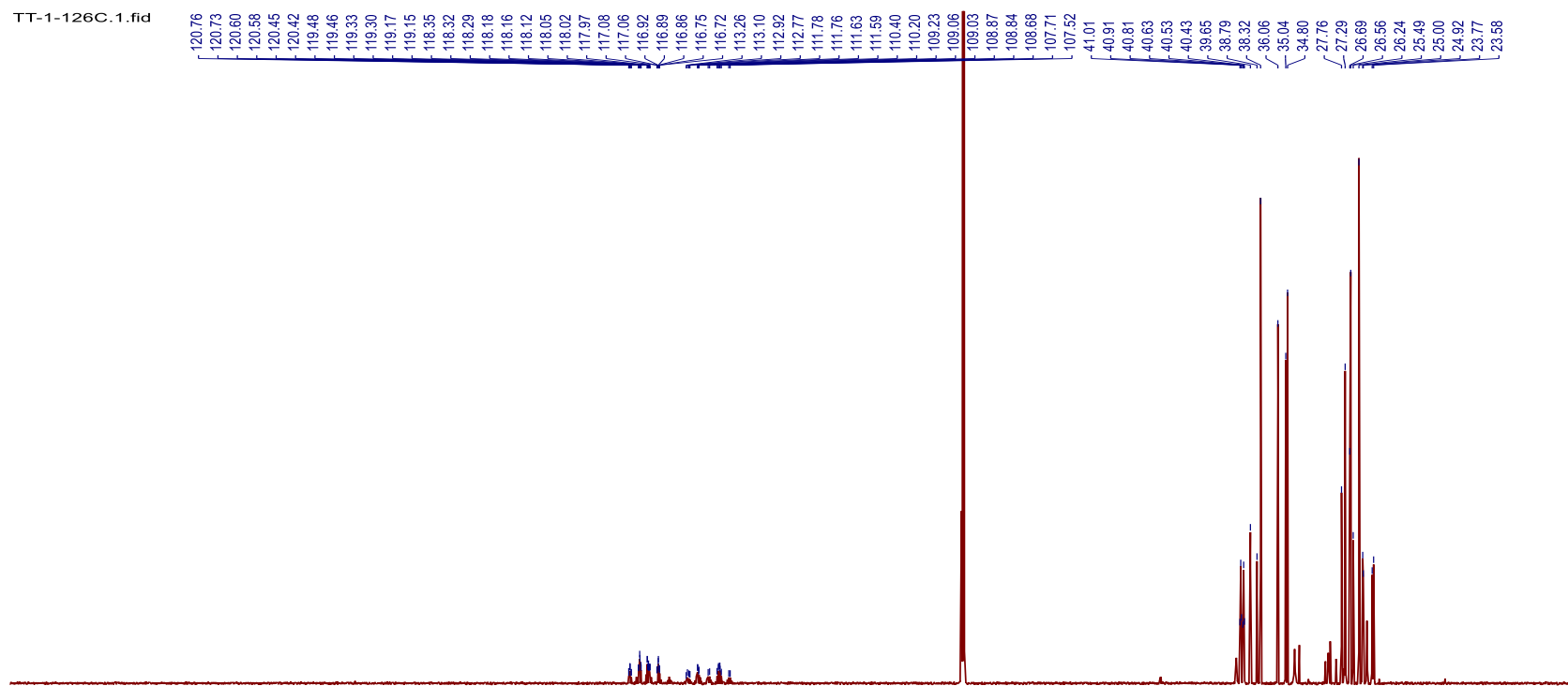

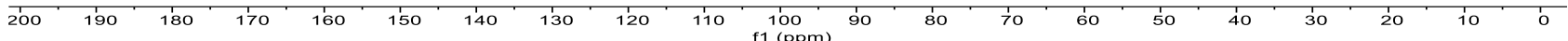

${ }^{19}$ F NMR: $\left(376 \mathrm{MHz}, \mathrm{CDCl}_{3}\right)$

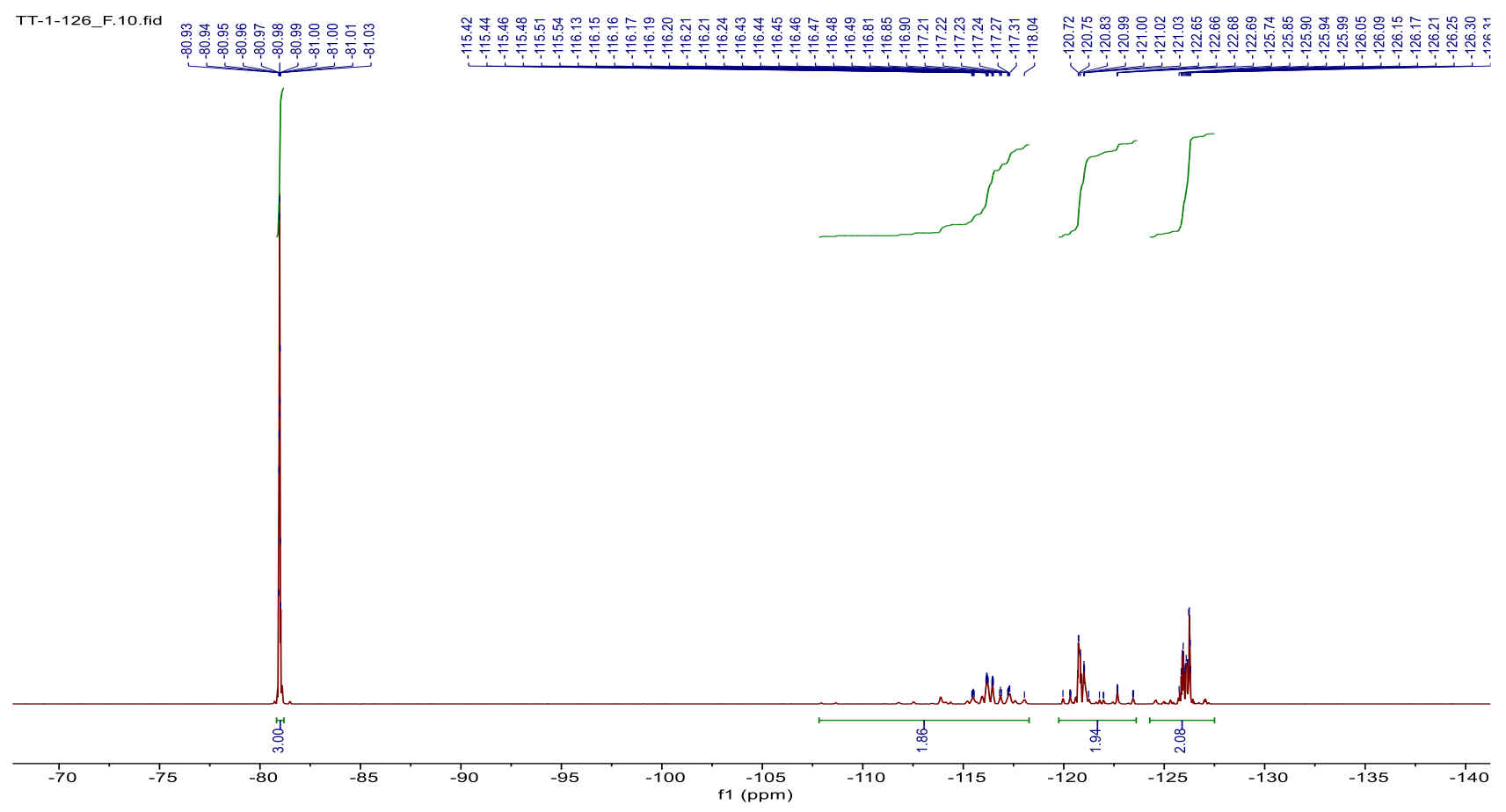



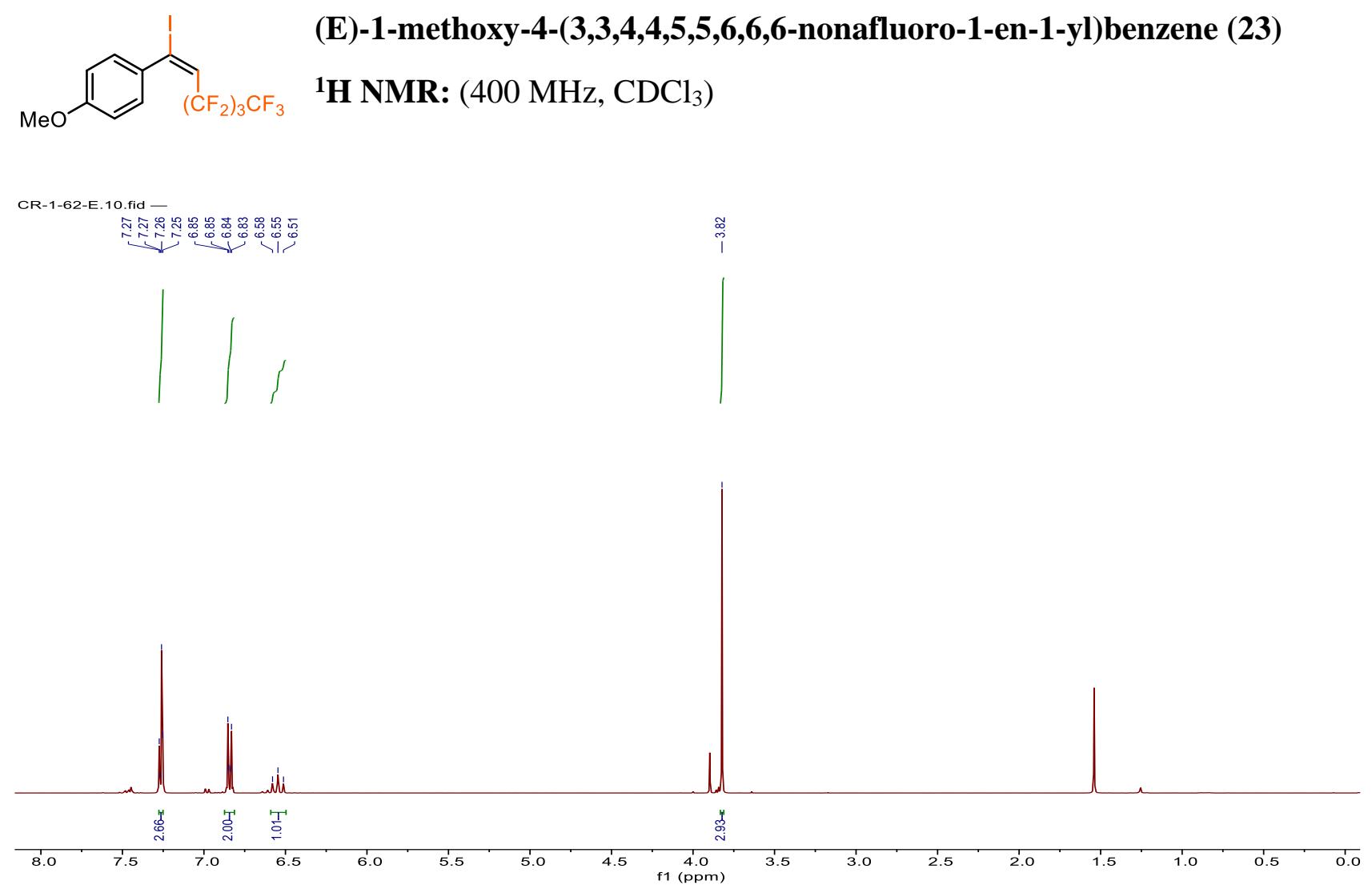

${ }^{13}$ C NMR: (201 MHz, $\mathrm{CDCl}_{3}$ )
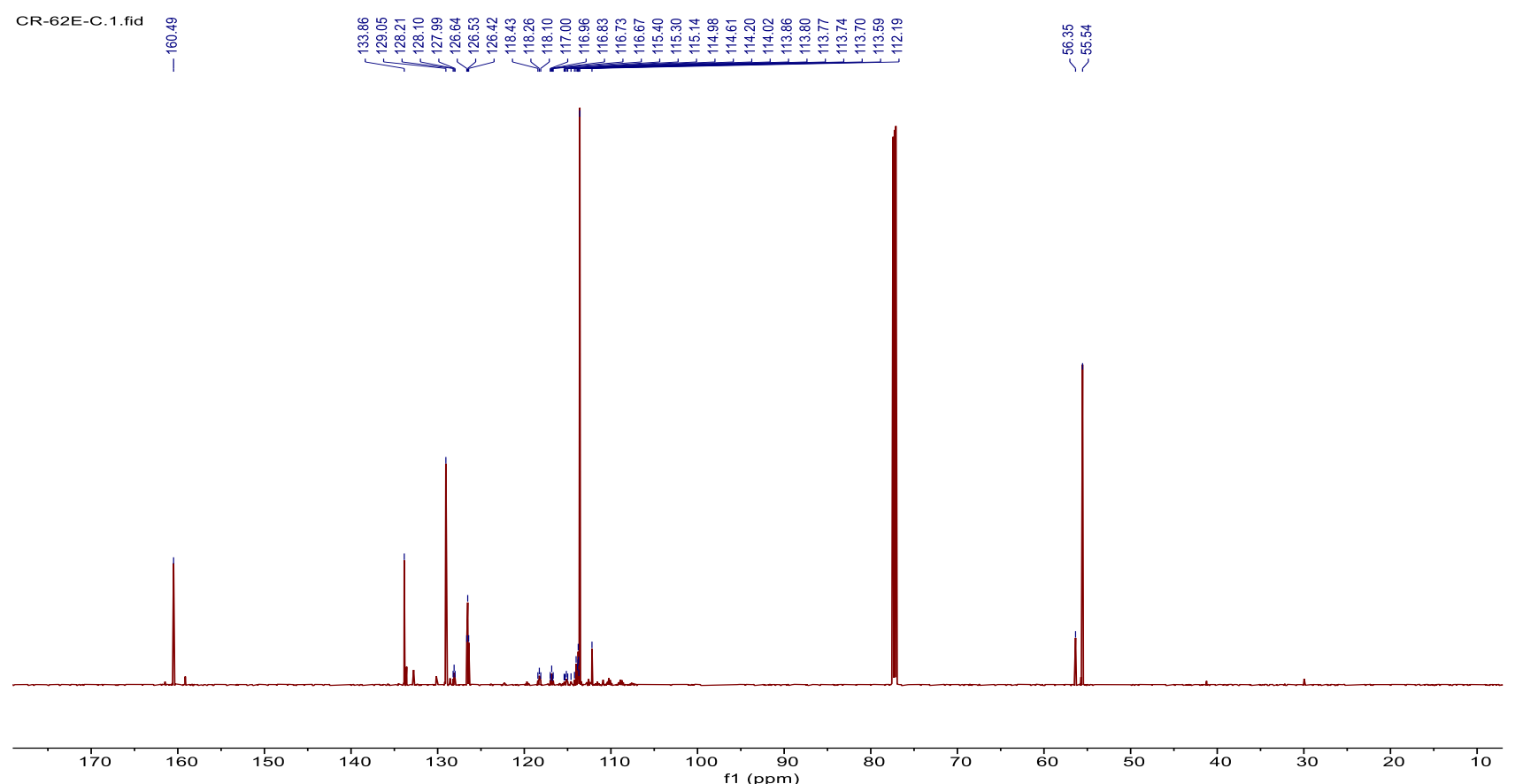

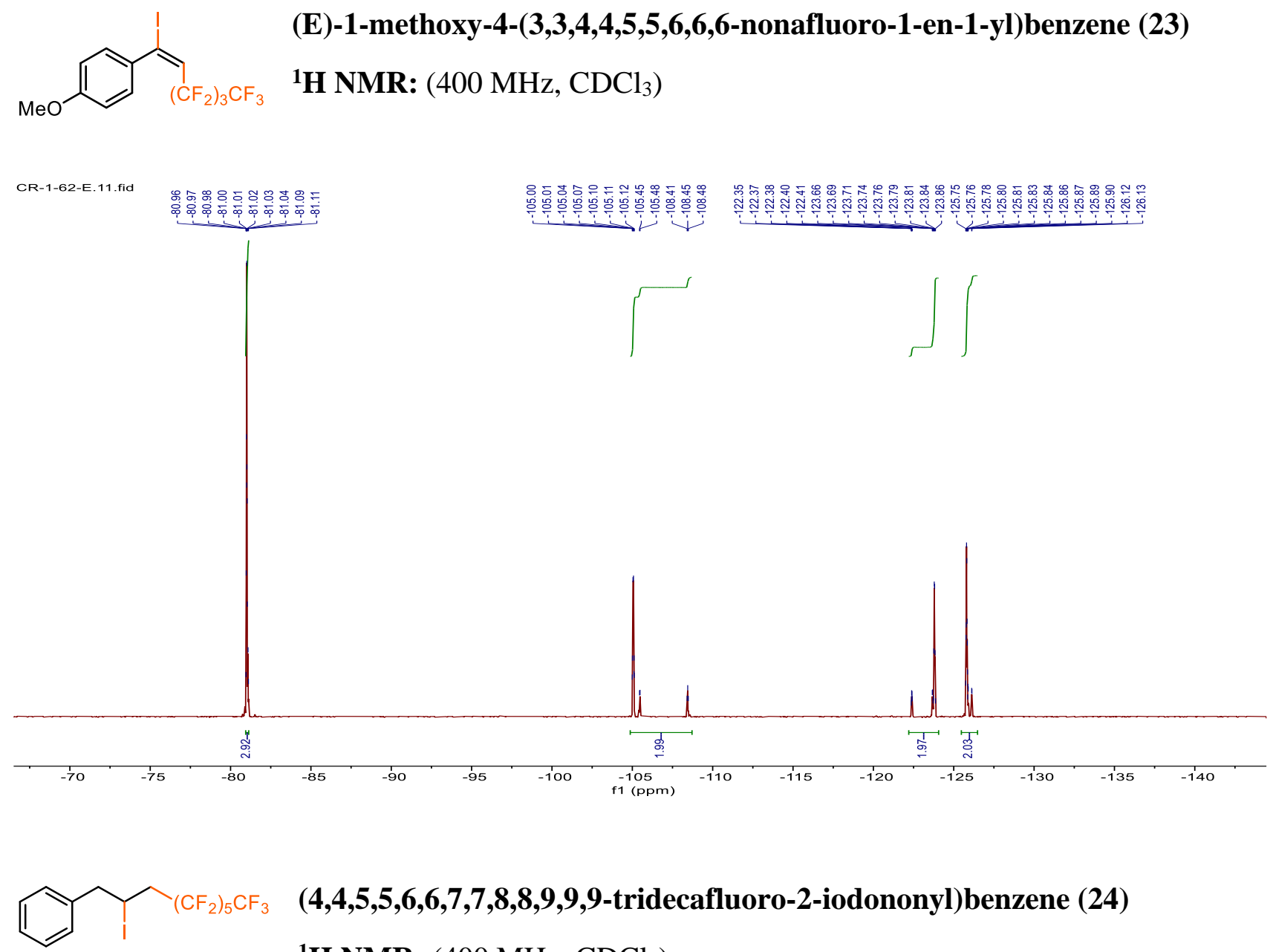

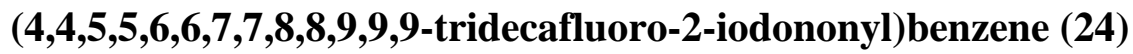
${ }^{1}$ H NMR: (400 MHz, $\mathrm{CDCl}_{3}$ )

Note: Compound is extremely hygroscopic. $\mathrm{H}_{2} \mathrm{O}$ could not be fully removed even after prolonged drying under high vacuum. CR-1-68.10.fid -

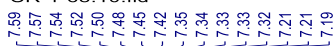

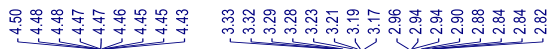
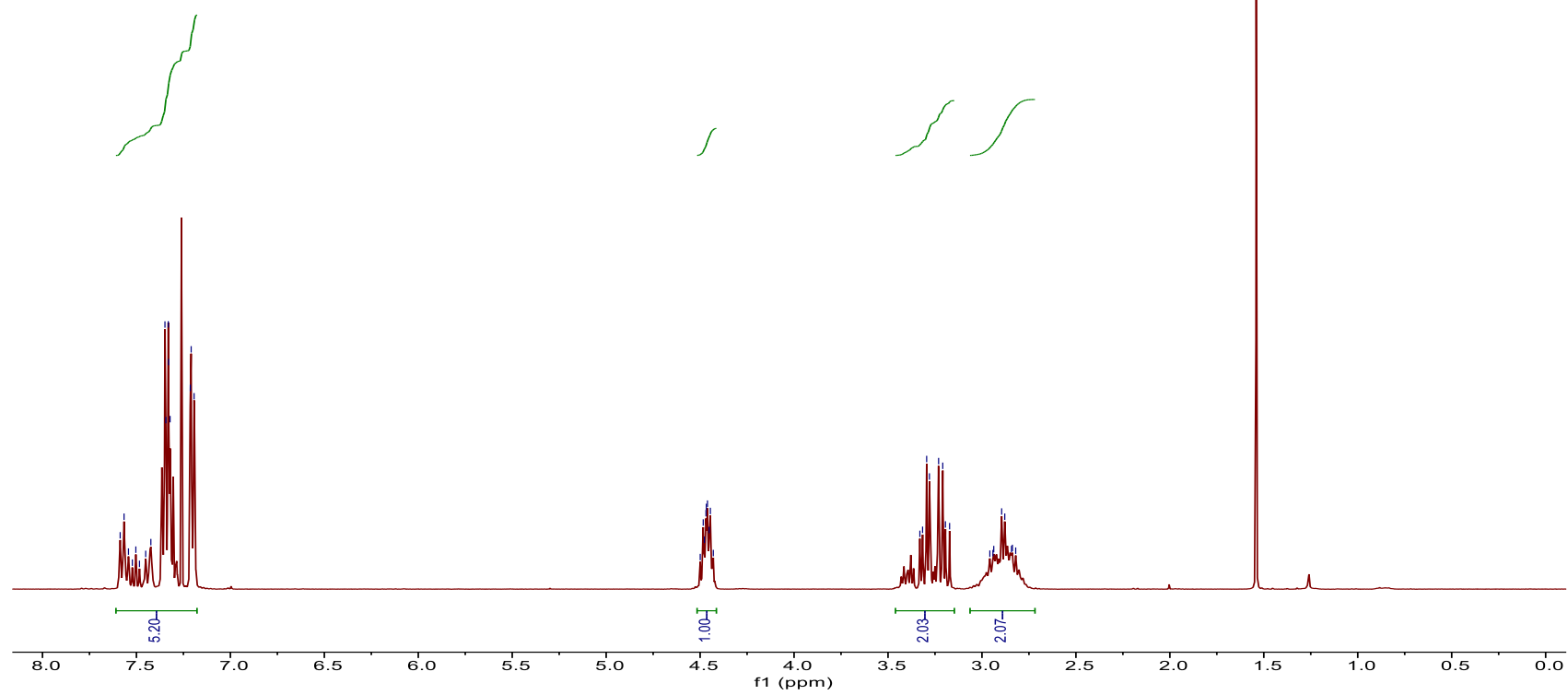


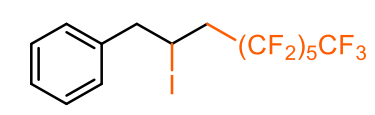

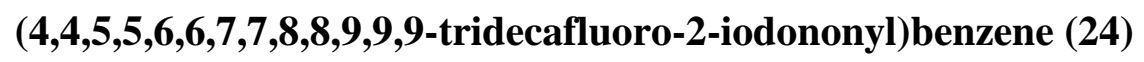

${ }^{13}$ C NMR: (201 MHz, $\left.\mathrm{CDCl}_{3}\right)$

CR-68C-re.1.fid
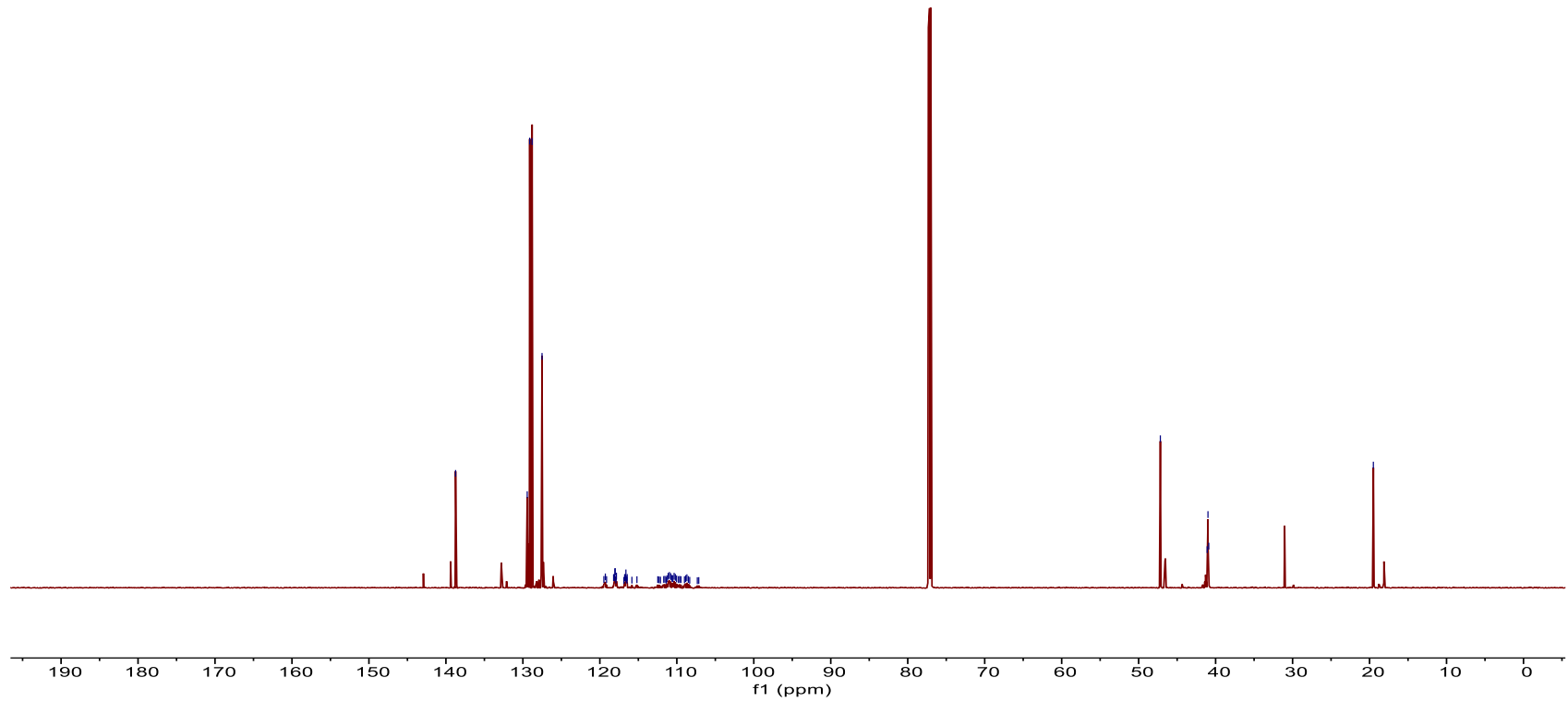

${ }^{19}$ F NMR: (376 MHz, $\left.\mathrm{CDCl}_{3}\right)$

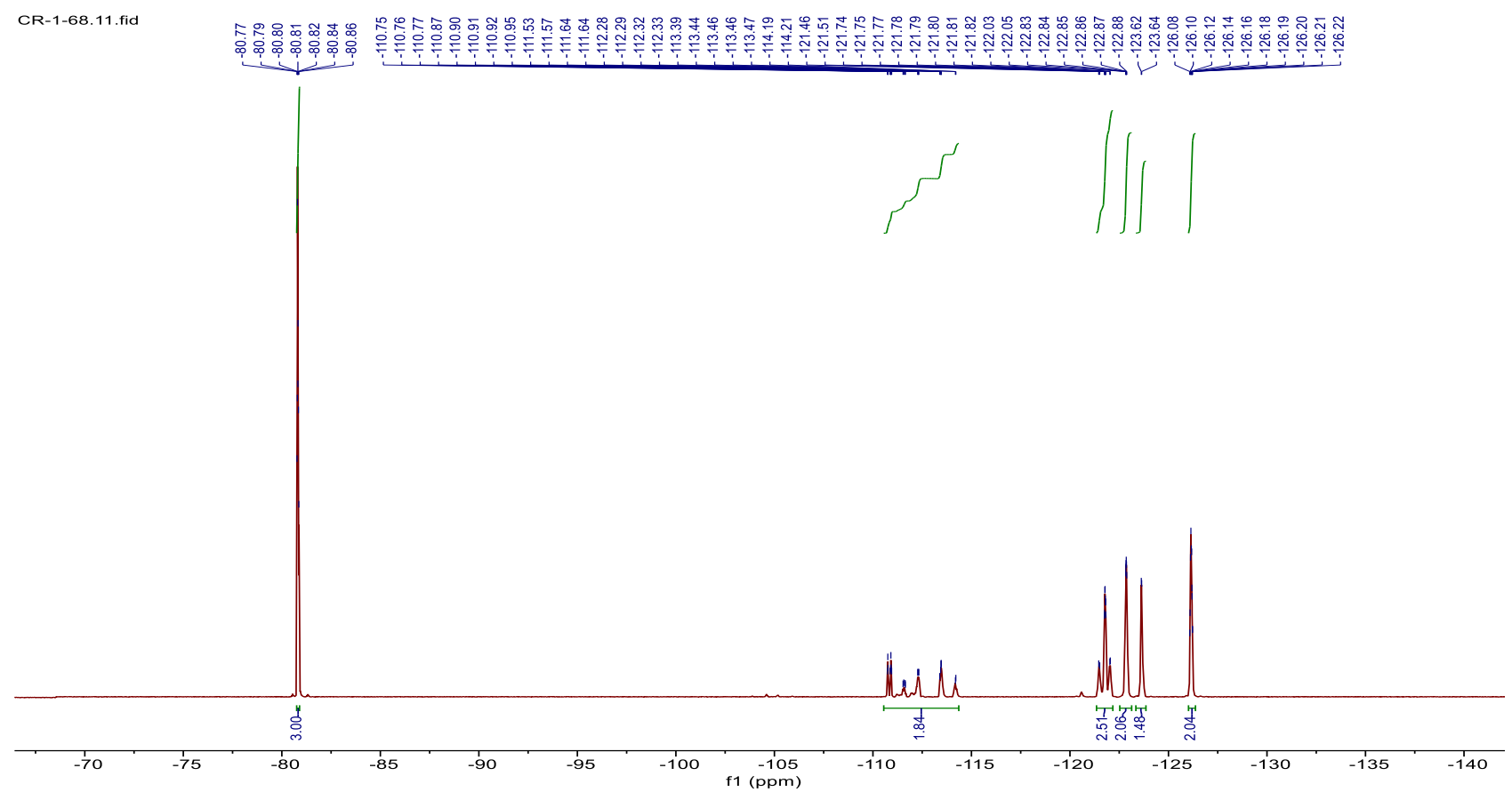




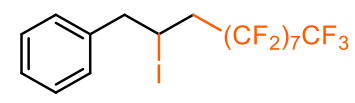

\section{$(4,4,5,5,6,6,7,7,8,8,9,9,10,10,11,11,11$-heptadecafluoro-2-iodoundecyl)- benzene (25)}

${ }^{\mathbf{1}}$ H NMR: (400 MHz, $\mathrm{CDCl}_{3}$ )

Note: Compound is extremely hygroscopic. $\mathrm{H}_{2} \mathrm{O}$ could not be fully removed even after prolonged drying under high vacuum.

CR-1-60.10.fid-

垶昌昌昌昌等
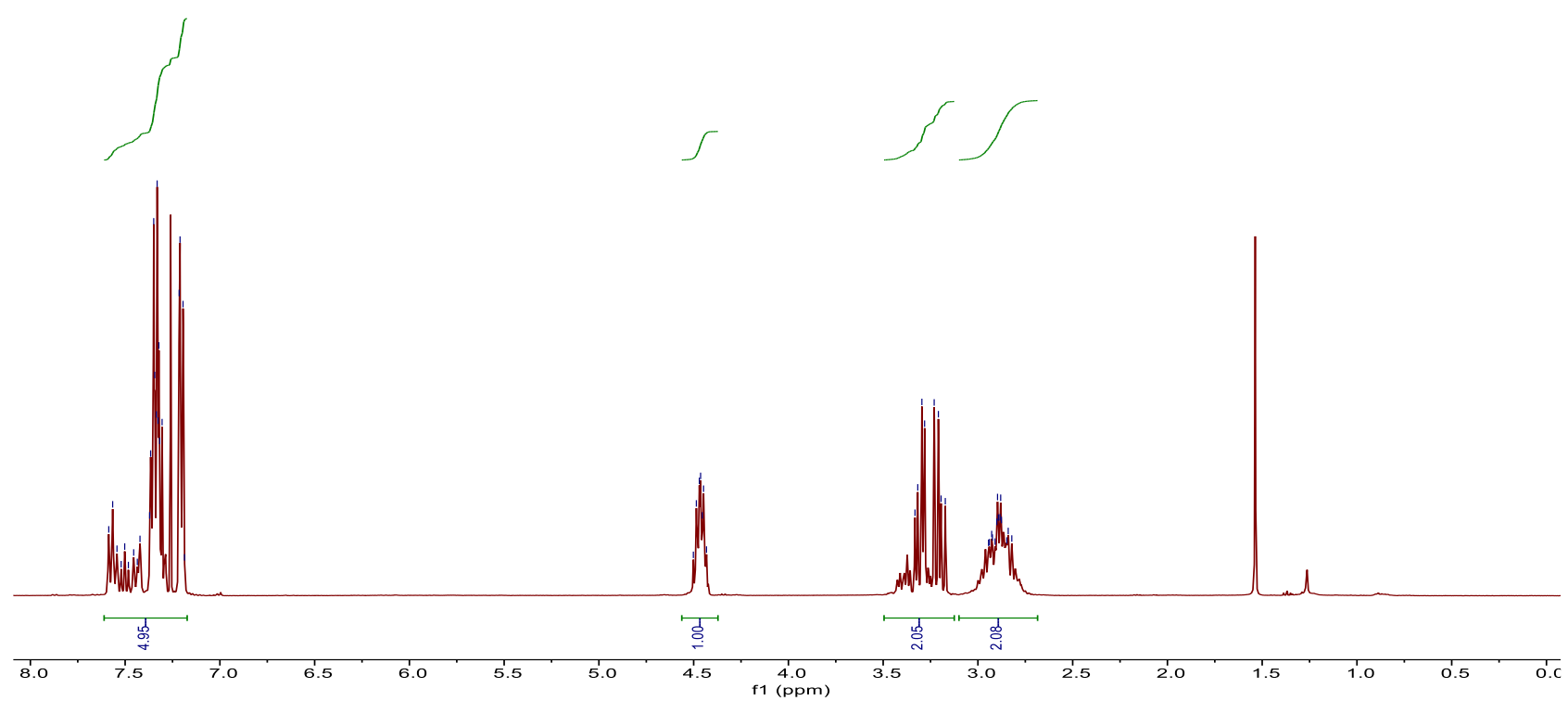

${ }^{13}$ C NMR: (201 MHz, $\left.\mathrm{CDCl}_{3}\right)$

CR-60C.1.fid

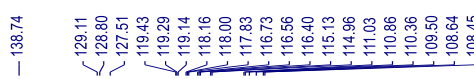

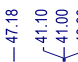

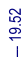

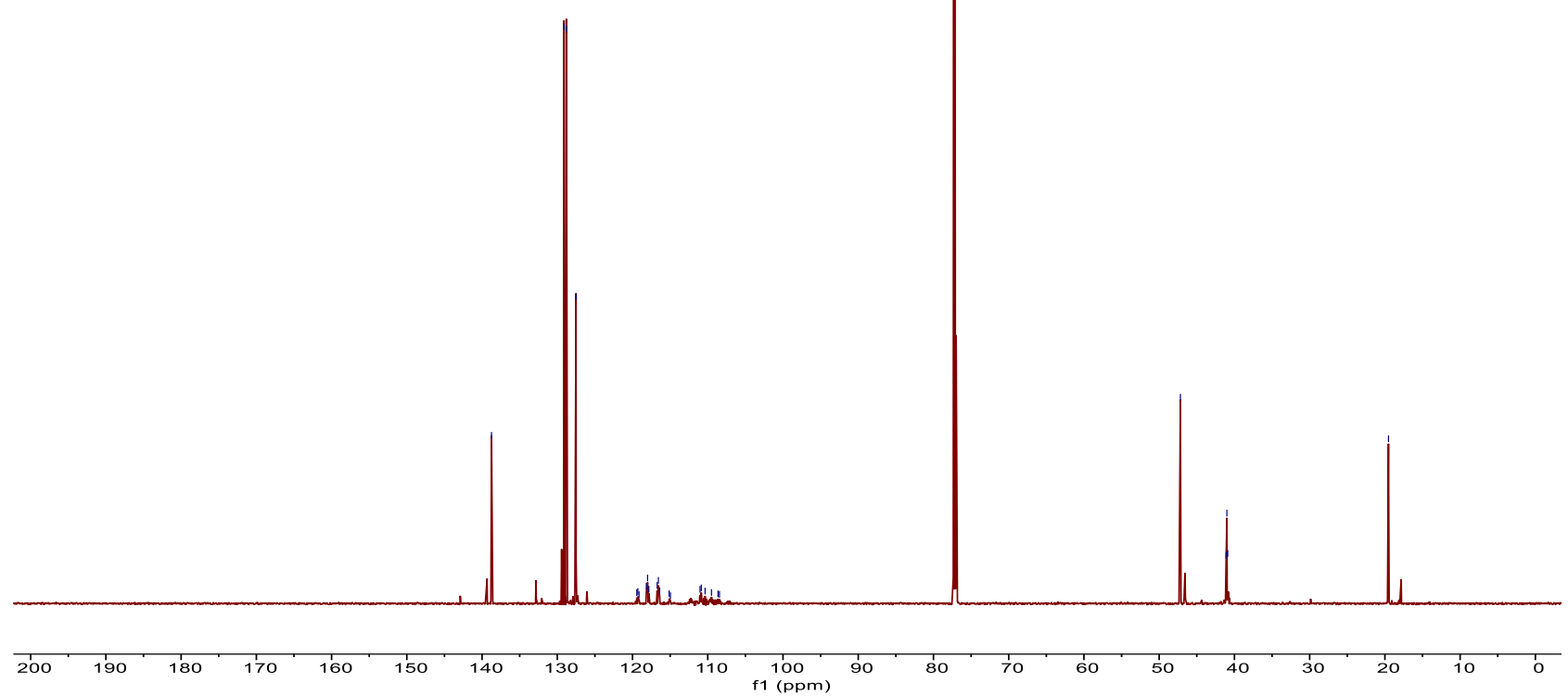




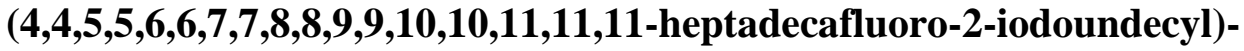
benzene (25)

${ }^{19}$ F NMR: $\left(376 \mathrm{MHz}, \mathrm{CDCl}_{3}\right)$

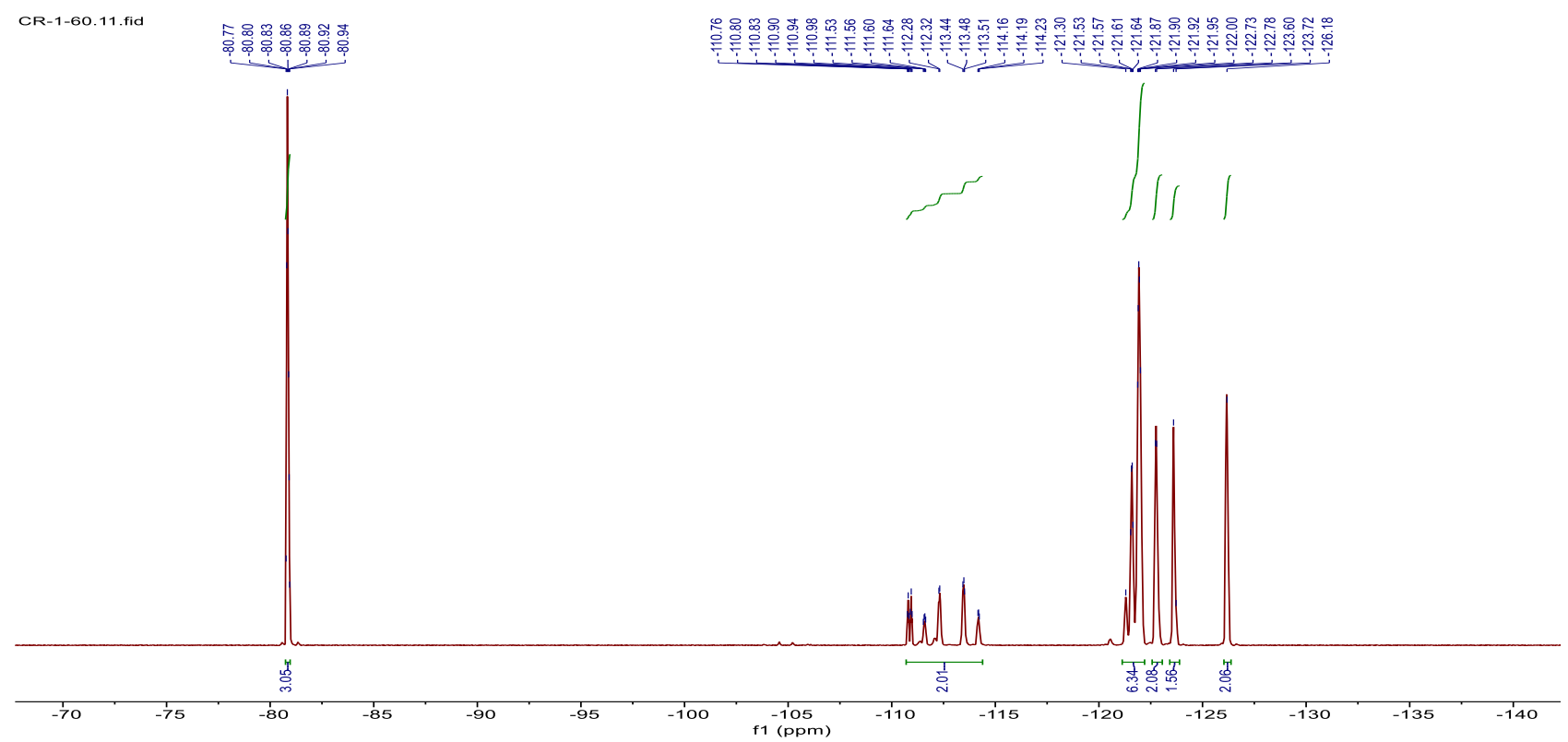

Ethyl 2,2-difluoro-4-iodo-5-phenylpentanoate (26)

${ }^{1}$ H NMR: $\left(400 \mathrm{MHz}, \mathrm{CDCl}_{3}\right)$

CR-1-58.1. fid -

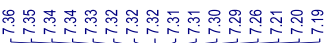

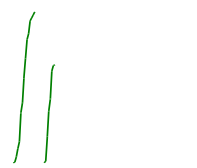

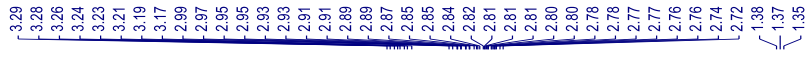

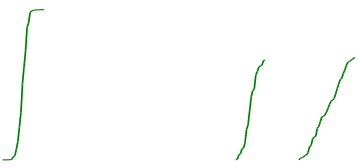

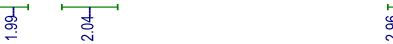

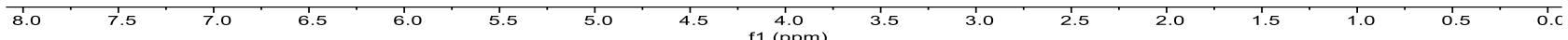




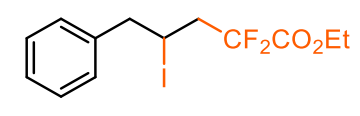

\section{Ethyl 2,2-difluoro-4-iodo-5-phenylpentanoate (26)}

${ }^{13}$ C NMR: (101 MHz, $\left.\mathrm{CDCl}_{3}\right)$

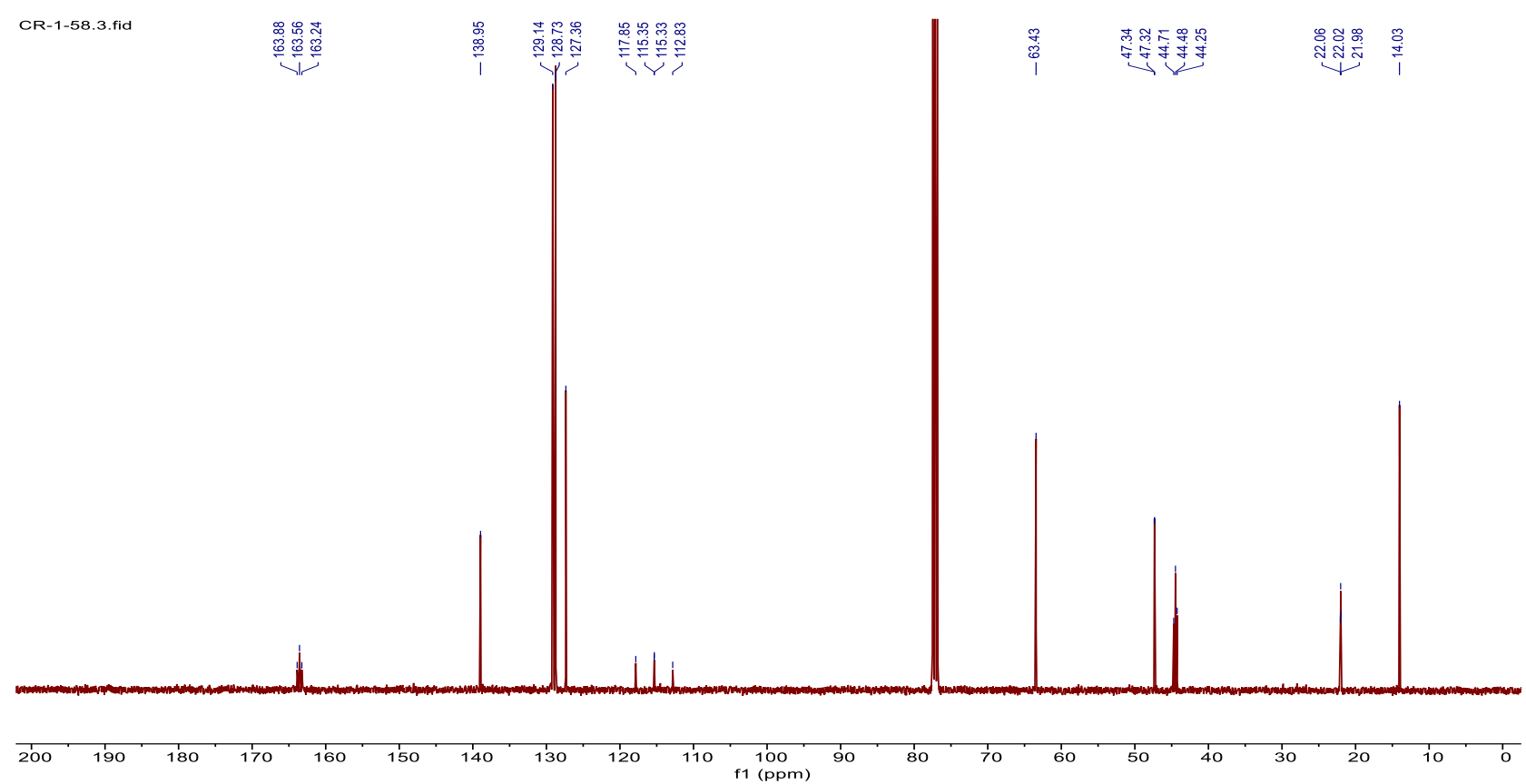

${ }^{19}$ F NMR: $\left(376 \mathrm{MHz}, \mathrm{CDCl}_{3}\right)$

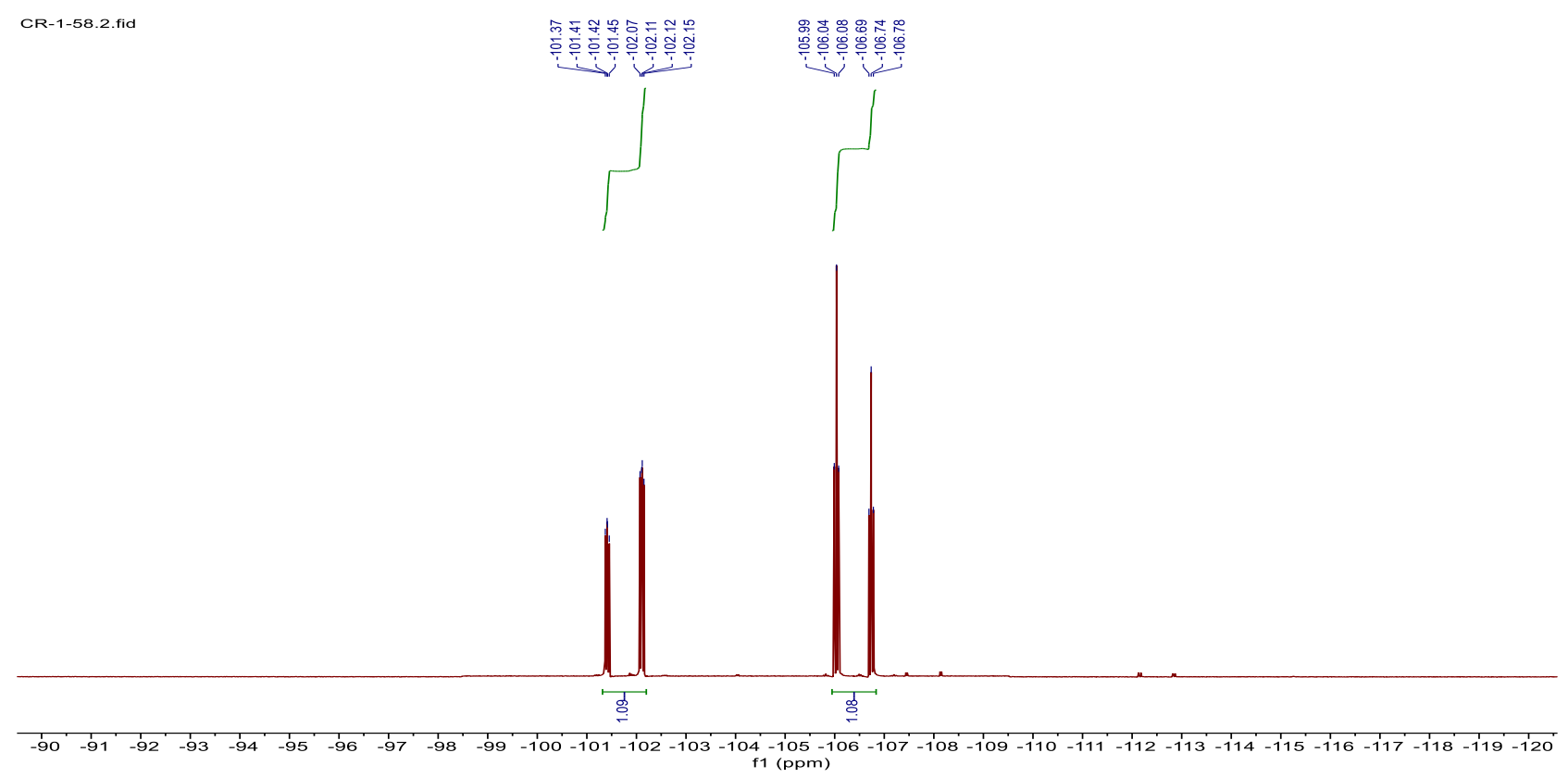

